\title{
Diffractive optical lens design and fabrication for integrated monitoring of microelectromechanical lateral comb resonators
}

\author{
William Brent McCormick \\ West Virginia University
}

Follow this and additional works at: https://researchrepository.wvu.edu/etd

\section{Recommended Citation \\ McCormick, William Brent, "Diffractive optical lens design and fabrication for integrated monitoring of microelectromechanical lateral comb resonators" (2004). Graduate Theses, Dissertations, and Problem Reports. 1551. \\ https://researchrepository.wvu.edu/etd/1551}

This Thesis is protected by copyright and/or related rights. It has been brought to you by the The Research Repository @ WVU with permission from the rights-holder(s). You are free to use this Thesis in any way that is permitted by the copyright and related rights legislation that applies to your use. For other uses you must obtain permission from the rights-holder(s) directly, unless additional rights are indicated by a Creative Commons license in the record and/ or on the work itself. This Thesis has been accepted for inclusion in WVU Graduate Theses, Dissertations, and Problem Reports collection by an authorized administrator of The Research Repository @ WVU. For more information, please contact researchrepository@mail.wvu.edu. 


\title{
Diffractive Optical Lens Design and Fabrication for Integrated Monitoring of Microelectromechanical Lateral Comb Resonators
}

\author{
by \\ William Brent McCormick \\ B.S.E.E, West Virginia University \\ Thesis submitted to the \\ College of Engineering and Mineral Resources at \\ WEST VIRGINIA UNIVERSITY \\ in partial fulfillment of the requirements for the degree of \\ Masters of Science \\ in \\ Electrical Engineering
}

\section{Committee members:}
Dr. Lawrence A. Hornak, Committee Chairperson
Dr. Parviz Famouri
Dr. Dimitris Korakakis

Lane Department of Computer Science and Electrical Engineering

\author{
Morgantown, West Virginia \\ 2004
}

Keywords: Diffractive optical elements, DOE, Microelectromechanical, MEMS 


\title{
Abstract
}

\section{Diffractive Optical Lens Design and Fabrication for Integrated Monitoring of Microelectromechanical Lateral Comb Resonators}

\author{
William B. McCormick
}

Analysis of fault characteristics and control of microelectromechanical systems (MEMS) is becoming increasingly important as these devices are incorporated into safety critical systems. Mechanically decoupled monitoring techniques are needed to ensure that accurate, low-noise positional information is gathered from these devices during operation. Optical monitoring has been shown by various groups to be capable of achieving this goal. However, Integrated Optical Monitoring (IOM) must be achieved in order to enable incorporation with the MEMS die in a single package for effective lifetime system monitoring. This research addresses the design, fabrication and testing of diffractive optical elements (DOEs) for achieving IOM.. DOEs for on and off-axis single and multiple beam generation are modeled based on a linear optical system model paraxial gaussian beam theory.

The DOEs are generated using a MATLAB tool that was completed as part of this work to calculate the desired input wave, output wave, and transfer function. This data is then used to generate a phase lens profile with an etch depth that is dependent on the material that will be used for lens fabrication. DOEs are fabricated on a borosilicate substrate and testing of the lenses is performed using a beam profiler to determine the number, shape and power of the probe beams for comparison with the results from the simulated lenses. Experimental DOE test results compare well with those obtained from the modeling tool validating the use of the DOE design tool and the fabrication process for subsequent research implementing IOM architectures. 


\section{Acknowledgements}

I would like to thank my committee members, Dr. Larry Hornak, Dr. Parviz Famouri, and Dr. Dimitris Korakakis for their support and guidance during my graduate research. I would also like to thank the members of the WVU MEMS research group, particularly Dr. Jeremy Dawson, Scott Rittenhouse, Rhaguveer Medipalli, Swetha Katipalli and Limin Wang for their insight and encouragement throughout this endeavor. I also want to thank Shantanu Pathak, Divya Pisharoty, Tim Cornell, and Dr. Kolin Brown for their assistance during my experimental work.

Most importantly, I want to thank my family for their patience and encouragement as I traveled this road. I especially want to thank my wife Teresa for her love, support and faith in me these past years. I also want to thank God for being with me during the trying times and the joyous times. 


\section{Contents}

\section{Diffractive Optical Lens Design and Fabrication for Integrated Monitoring of} Microelectromechanical Lateral Comb Resonators ............................................................ i

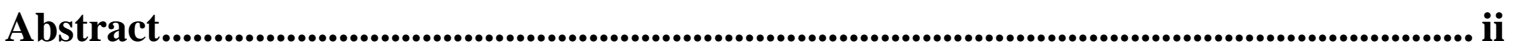

Acknowledgements ............................................................................................................................ iii

Contents .................................................................................................................................. iv

Table of Figures and Tables....................................................................................................... vi

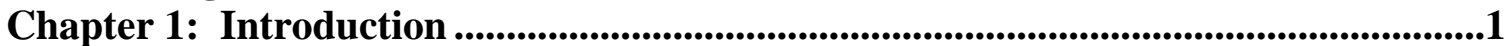

1.1 Overview of Current MEMS Devices and Uses ......................................................... 1

1.2 Current Limitations of MEMS Actuator Use........................................................ 2

1.2.1 Monitoring Methods ................................................................................ 2

1.2.2 Quality Assurance/Fault Detection .................................................................... 3

1.3 Introduce proposed method (integrated optics/off-axis optics) ................................... 4

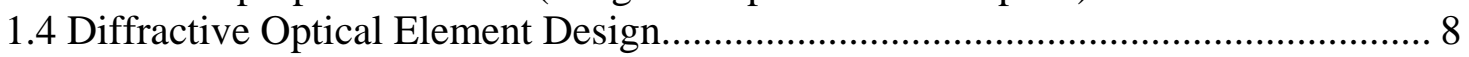

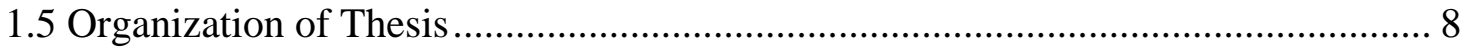

Chapter 2: Theory.................................................................................................................10

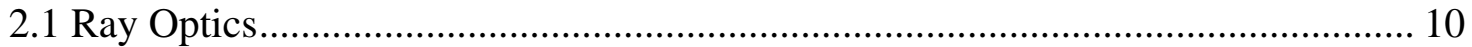

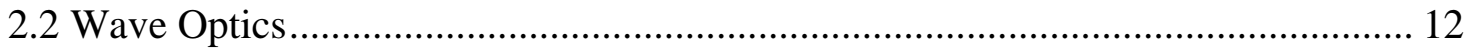

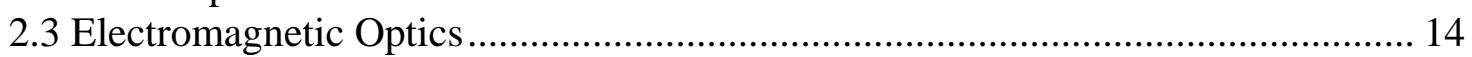

2.4 Interference and Diffraction................................................................................. 17

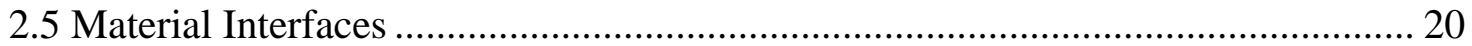

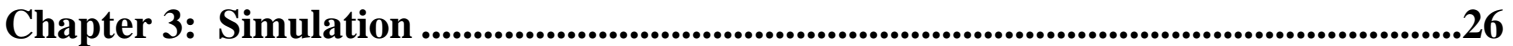

3.1 Lens Generation Method.................................................................................. 26

3.1.1 Gaussian Beam Input/Output...................................................................... 28

3.1.2 General Transfer Function of Free space....................................................... 29

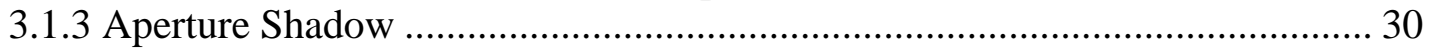

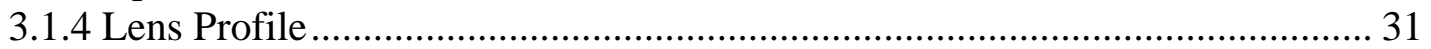


3.1.5 Conversion from Continuous to Binary ..................................................... 31

3.2 On-Axis and Off-Axis DOE Generation and Simulation .................................... 32

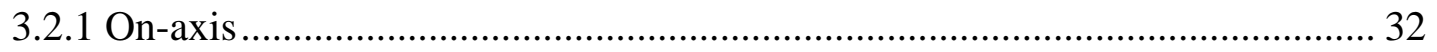

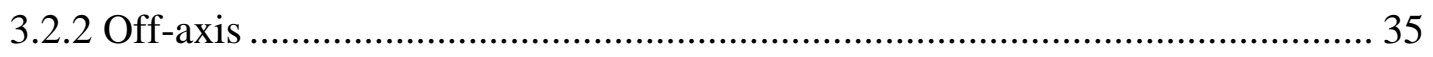

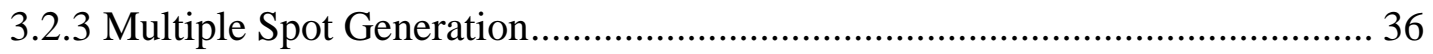

3.3 Conversion from Simulation to Mask File....................................................... 37

Chapter 4: Fabrication and Characterization of DOE lens designs .............................39

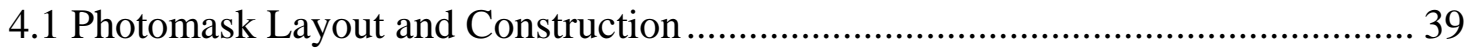

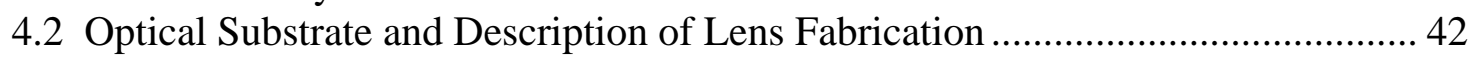

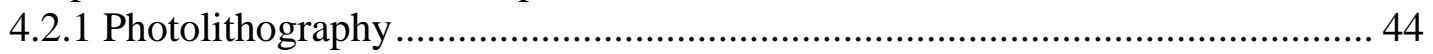

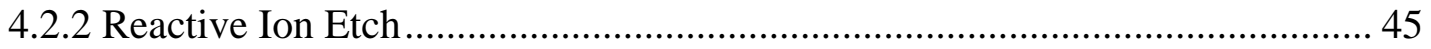

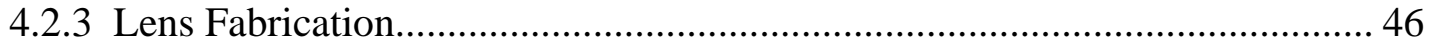

4.3 Bulk Optical Tests of Lens Design ................................................................... 58

Chapter 5: Conclusion and Future Investigation ...........................................................72

Appendix A: Lens Design Programs ...............................................................................74

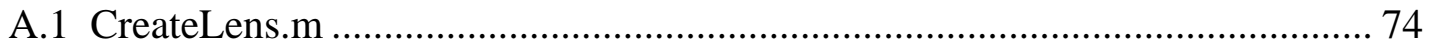

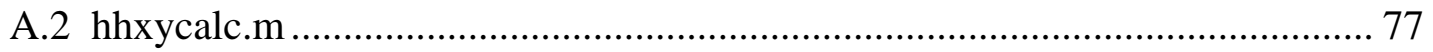

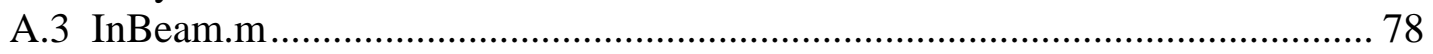

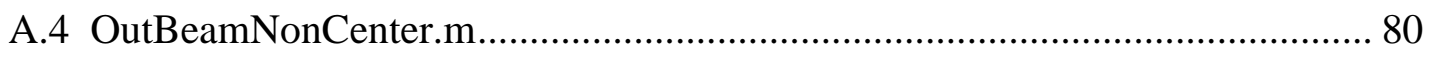

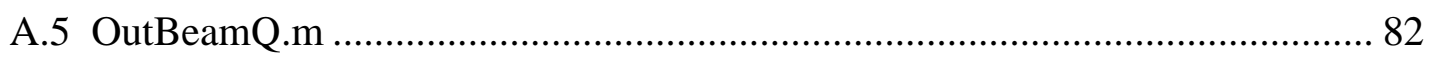

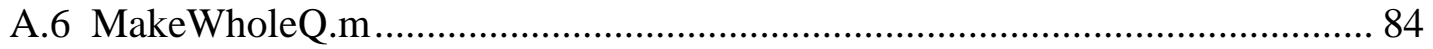

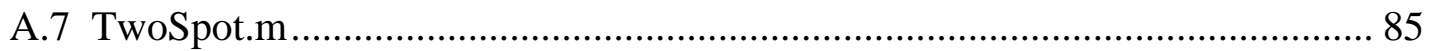

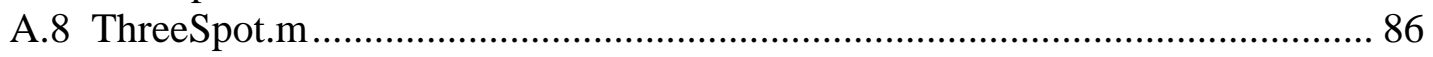

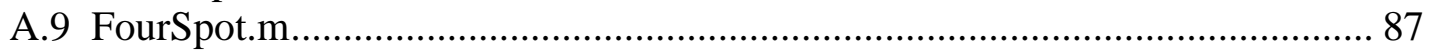

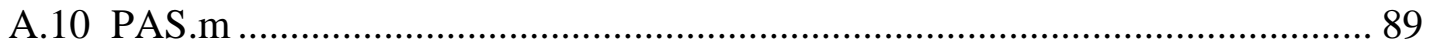

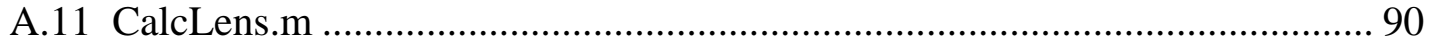

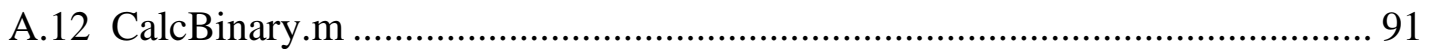

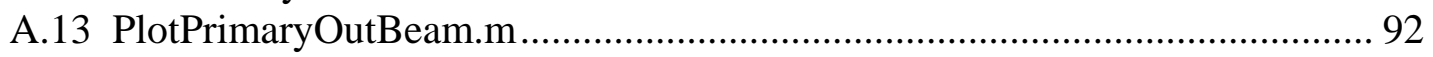

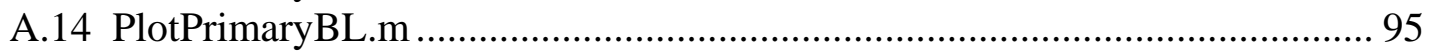

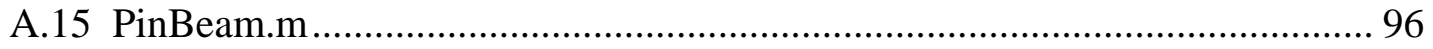

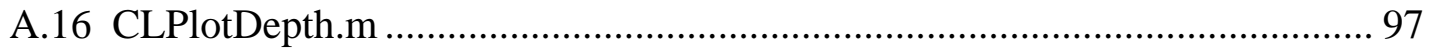

Appendix B: Lens Testing Programs ...............................................................................98

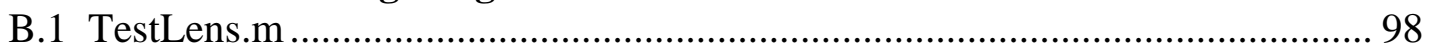

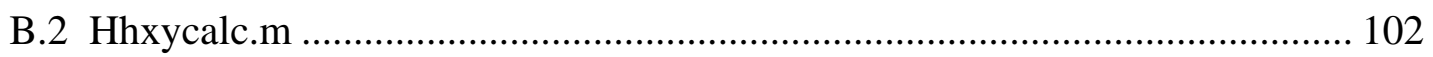

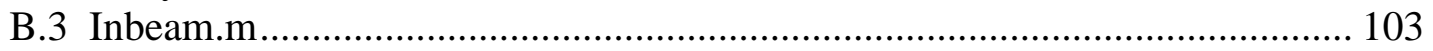

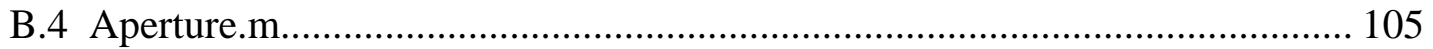

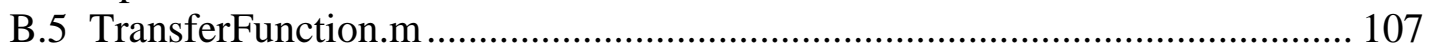

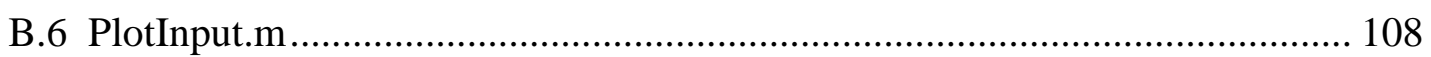

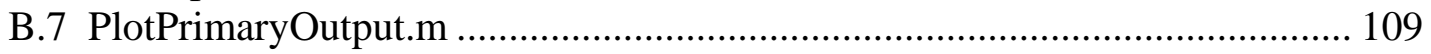

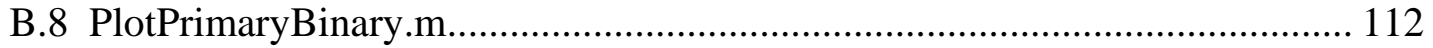

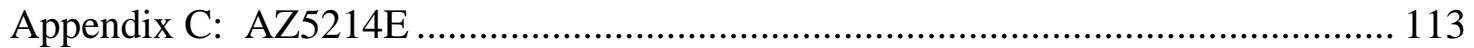

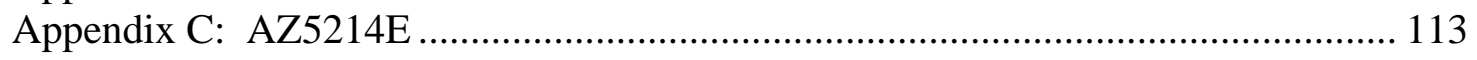




\section{Table of Figures and Tables}

Figure 1.1: Integrated opto-electrical MEMS package. ............................................ 4

Figure 1.2: Proposed off-axis opto-electrical MEMS substrate.....................................5 5

Figure 1.3: Proposed on-axis opto-electrical MEMS substrate................................6 6

Figure 1.4: $\mathrm{Cr} / \mathrm{Au}$ electrical traces on current iteration of opto-electrical substrate [12]

Figure 1.5: Current electrical packaging with optical probe fiber. ............................. 7

Figure 2.1: Example of Snell's Law................................................................... 11

Figure 2.2: Gaussian Beam [17] .......................................................................... 14

Figure 2.3: Water wave example of diffraction [24]..................................................... 18

Figure 2.4: Validation of use of paraxial approximation........................................... 19

Figure 2.5: Reflected and transmitted beams at planar dielectric boundary. ......... 21

Figure 2.6: Transmittance through the thin films and semi-infinite layers of current

MEMS LCR............................................................................................................... 24

Figure 2.7: Equivalent thin film and semi-infinite layers by using characteristic

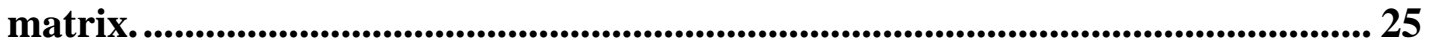

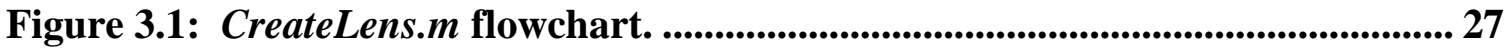

Figure 3.2: TestLens.m flowchart. ........................................................................ 28

Figure 3.3: Generated input and output beams. ............................................................. 29

Figure 3.4: Transfer Function. ............................................................................... 30

Figure 3.5: Central region of dual spot on-axis continuous profile............................ 32

Figure 3.6: Central region of dual spot on-axis binary profile................................ 32

Figure 3.7: Normalized intensity plots of input Gaussian beam used for DOE

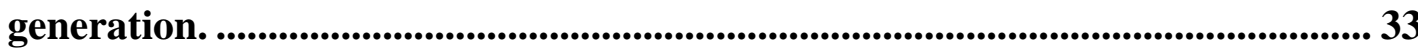

Figure 3.8: Normalized intensity plot of desired on-axis $10 \mu \mathrm{m}$ diameter Gaussian probe beam. ........................................................................................................... 34

Figure 3.9: Generated single spot on-axis binary DOE......................................... 34 
Figure 3.10: Normalized intensity plot of desired off-axis $10 \mu \mathrm{m}$ diameter Gaussian probe beam shifted $300 \mu \mathrm{m}$ from center of focal plane. .................................... 35

Figure 3.11: Generated single spot on-axis binary DOE.......................................... 36

Figure 3.12: Normalized intensities of desired multiple Gaussian outputs. ............. 37

Figure 3.13: Binary dual-spot DOEs................................................................................ 37

Figure 4.1: L-Edit layout of DOE optical photolithography test mask. ................... 40

Table 4.1: Location of DOEs by type. ........................................................................ 40

Figure 4.3: Chemical composition of Borofloat [27]..................................................... 42

Figure 4.4: Borofloat transmittance vs. IR wavelength [29] .................................. 43

Figure 4.5: Borofloat transmittance vs. UV wavelength [28].................................. 43

Figure 4.6: Borofloat index of refraction vs. wavelength [30] ............................... 44

Figure 4.7: Positive and negative photolithography........................................... 45

Figure 4.8: Generic image reversal photolithography and RIE etch process.......... 46

Figure 4.9: PR de-adhesion, lift-off and incomplete exposure................................ 48

Figure 4.10: Diffraction effects in small features during soft contact exposure. ..... 49

Figure 4.11: Sloped PR sidewalls............................................................................. 50

Table 4.2: Final photolithography process which yielded $0.5 \mu \mathrm{m}$ features. ............. 51

Figure 4.12: PR pattern containing $0.5 \mu \mathrm{m}$ features. .............................................. 52

Figure 4.13: Examples of trenching, micromasking and improper PR development resulting in sloped sidewalls................................................................................... 53

Figure 4.14: Sloped sidewalls and evidence of diffraction effects during photolithography. Debris in middle of trenches is believed to be non-volatile Fluorine compounds. ..................................................................................... 55

Figure 4.15: Evidence of chemical etching of $\mathrm{SiO}_{2}$ and $\mathrm{B}_{2} \mathrm{O}_{3}$ leaving behind nonvolatile debris.......................................................................................... 56

Figure 4.16: Nano-scale Fluorine compound structures. ...................................... 56

Figure 4.17: Conversion to $\mathrm{SF}_{6}$ process gas and modified RIE recipe shows marked improvement in sidewalls and removal of debris............................................. 58

Table 4.3: Spiricon ND filter transmission values [37]............................................. 59

Figure 4.18: Schematic of experimental setup. .................................................... 59

Figure 4.19: Portion of the letter " $L$ " used to determine amount of magnification

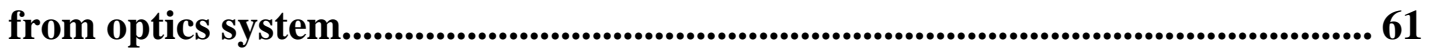

Table 4.4: Peak power and beam size. ...............................................................62 62

Table 4.5: Approximate output beam sizes. .........................................................62 62

Table 4.6: Percent error of experimental DOE focal beam size vs. desired size of 10

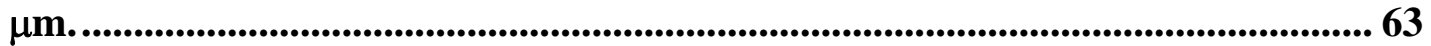

Figure 4.20: Experimental measurement of output from C3R2.............................. 64

Figure 4.21: Input beam routed through two beamsplitters....................................... 65

Figure 4.22: Input beam without intervening optical components. ........................... 65

Figure 4.23: 3D experimental output from C3R2 .................................................66

Figure 4.24: 3D simulated output from C3R2 .....................................................66

Figure 4.25: Generated DOE profile for C3R4 .......................................................... 67

Figure 4.26: Simulated output of C3R4..............................................................................68 68

Figure 4.27: Photograph of experimental output from C3R4. ................................... 68

Figure 4.28: Desired focal points in the off-axis case...............................................69 69

Figure 4.29: Mirrored region outside of DOE........................................................69 
Figure 4.30: L-Edit layout of incorrect on-axis single probe DOE. ............................ 70

Figure 4.31: Magnified view of central output intensity of incorrect DOE.............. 71 Figure 4.32: Experimental data from incorrect DOE patterned in PR. Notice correlation with simulation output shown in Figure 4.31 ............................... 71 


\section{Chapter 1: Introduction}

\subsection{Overview of Current MEMS Devices and Uses}

Microelectromechanical systems (MEMS) are micron-scale devices incorporating both mechanical and electrical characteristics and are fabricated using techniques and materials similar to those used in the electronics semiconductor industry. Building on the

advances made by the semiconductor industry allowed MEMS research to grow quickly by utilizing photolithographic techniques and information of materials properties garnered by the integrated chip (IC) industry. Current materials used in MEMS fabrication can range from single crystal silicon and silicon oxides to metals and III-V materials while the fabrication processes can be wet bulk micromachining, surface micromachining, LIGA and others depending on the materials used [1].

The basic fabrication process of the majority of MEMS devices utilizes some form of photolithography for pattern transfer followed by either an etch or deposition. Wet bulk micromachining takes advantage of the crystalline structure of the substrate, usually $\mathrm{Si}$, to wet etch patterns directly into the die with subsequent depositions and pattern etches [1]. Another example is the LIGA (X-ray Lithographie Galvanoformung Abformtechnik) process which uses thick layers of photoresist (PR), from microns to centimeters, and deep X-ray lithography to create PR molds that are filled with metal using electrodeposition which are either the final product or used as molds for plastic injection 
molding [1]. Surface micromachining is generally performed by depositing thin layers of desired material onto a substrate via LPCVD (Low-Pressure Chemical Vapor Deposition), MOCVD (Metal Oxide Chemical Vapor Deposition) or PECVD (Plasma Enhanced Chemical Vapor Deposition) which are then patterned and etched using dry etch processes. Examples of surface micromachining processes are the five layer polysilicon SUMMiT V'M (Sandia Ultra-planar, Multi-level MEMS Technology 5) [2] process created at Sandia National Laboroatories and currently commercially marketed by MEMX [3], and the MUMPs® (Multi-User MEMS Processes) offered by MEMSCAP [4].

\subsection{Current Limitations of MEMS Actuator Use}

Integrated monitoring of MEMS actuators in critical applications such as arming mechanisms, biomedical devices, or space exploration is needed to determine the operational status of the device over its lifetime. Systems can degrade during operation from environmental conditions such as humidity, leading to oxide growth and/or stiction, from motional stress that can lead to fracturing of the high stress points (such as spring connections or anchor points) or from particulates such as dust or debris from other devices or package degradation. Methods for monitoring include electrical and optical techniques $[5,6,7,8]$.

\subsubsection{Monitoring Methods}

Electrical monitoring can be either capacitive or piezoresistive in nature, with the advantage of well known and established operational understanding as well as a mature fabrication industry in CMOS (Complementary Metal-Oxide semi-Conductor) processes. One of the disadvantages is the problem of signal-to-noise ratio arising from the relatively large voltages needed for actuation of MEMS devices compared to the capacitive signal received from device motion. Charging effects of oxide layers can also possibly alter operation of device.

Optical monitoring offers the advantage of being totally decoupled from the drive signal of the system, eliminating the introduction of noise to the signal from the voltage of the device drive signal. Optical monitoring approaches include those in which the 
optical beam interacts in transmission or reflection with the microstructure. In addition the optical power can be delivered via waveguide or free space.

Waveguide optics has the advantage of precise alignment if included in the initial design and fabrication of MEMS devices but is difficult to implement if the MEMS die requires a wet release process to remove sacrificial oxide layers [9]. This can be alleviated using alternate manufacturing and dry release processes such as those found in the ASIMPS (Application-Specific MEMS Process Service) project, a collaboration of MEMSCAP and Carnegie Mellon University [10]. ASIMPS is a combination CMOSMEMS process using a deep reactive ion etch (DRIE) procedure to release the MEMS structures. This process contains additional metal layers that could act as protective masks to prevent damage to the dielectric integrated waveguides.

Free space optics have the advantage of implementation after die release, removing the problems of damage from wet or dry release processes but this is balanced by increased complexity due to alignment issues. The through-wafer optical monitoring system is one example of a free space approach to monitor MEMS devices [11].

Both waveguide and free space optics suffer from a loss of power due to reflection and absorption from the MEMS structural layer interfaces and materials respectively.

\subsubsection{Quality Assurance/Fault Detection}

De-coupled monitoring of MEMS device motion over the lifetime of operation is necessary for devices operating in safety critical systems or systems that will encounter dynamic loads. Implementation of closed loop control and fault detection routines will contribute to more robust systems with greater flexibility and longer operational lifetime. An in situ probe method is required to further this goal of a self contained operational and monitoring system.

Current research being performed at West Virginia University (WVU) is focusing on closed loop feedback control and fault detection of lateral comb resonators (LCR) using a through-wafer optical probe for characterization of device parameters and continuous monitoring of device motion [12,13,14,15]. LCRs were designed with a $2 \mu \mathrm{m}$ pitch grating structure built into the shuttle that would interact with the through-wafer probe giving a positional resolution of $2 \mu \mathrm{m}$ of motion. "Faults" were also built into some of 
the LCRs in the form of extra poly layers for change in mass to simulate debris or missing comb fingers and changes in flexure length and width corresponding to changes in the spring constant which could result from broken or worn flexures.

The logical progression of research at WVU is implementation of an integrated optical monitoring package.

\subsection{Introduce proposed method (integrated optics/off-axis optics)}

The through-wafer optical probe system developed in our lab currently uses bulk optical methods to deliver and focus the beam on the Comb Resonator (LCR) that is being monitored. This system is effective but requires a large footprint to accommodate the optical and mechanical devices necessary for proper alignment and delivery and is limited to a 2 um resolution of device movement.

An integrated optical monitoring system is being proposed that would incorporate diffractive optical elements (DOEs) to replace the bulk optical delivery and monitoring system to reduce size and facilitate the development of a packaged system which could ultimately be fielded with the MEMS and monitor it over its lifetime of operation. Integrated DOEs also enable delivery of multiple probe beams to the device to increase positional resolution. Figure 1.1 is a graphical representation of an integrated optoelectrical MEMS monitoring system.

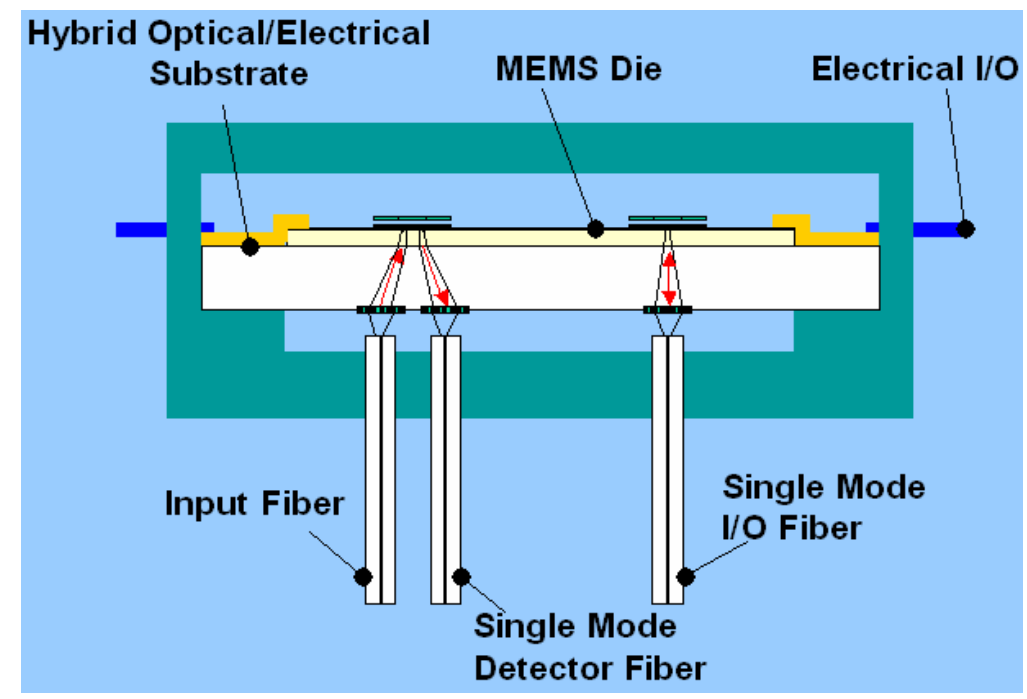

Figure 1.1: Integrated opto-electrical MEMS package. 
Figures 1.2 and 1.3 offer a more detailed diagram of one embodiment for integrating MUMPS fabricated MEMS LCR die with DOEs. Both figures include the dimensions of individual layers of the die, opto-electric substrate and external connections to provide power and the optical probes. Figure 1.2 presents a method of introducing an input laser beam via a single-mode optical fiber for generation of an off-axis probe beam with either single or multiple probe spots using a DOE. The DOE must be designed to create a focal point at the level of the diffraction grating fabricated into the shuttle of the LCR. The reflected wave from the shuttle would then be guided to a different collection fiber using a separate DOE.

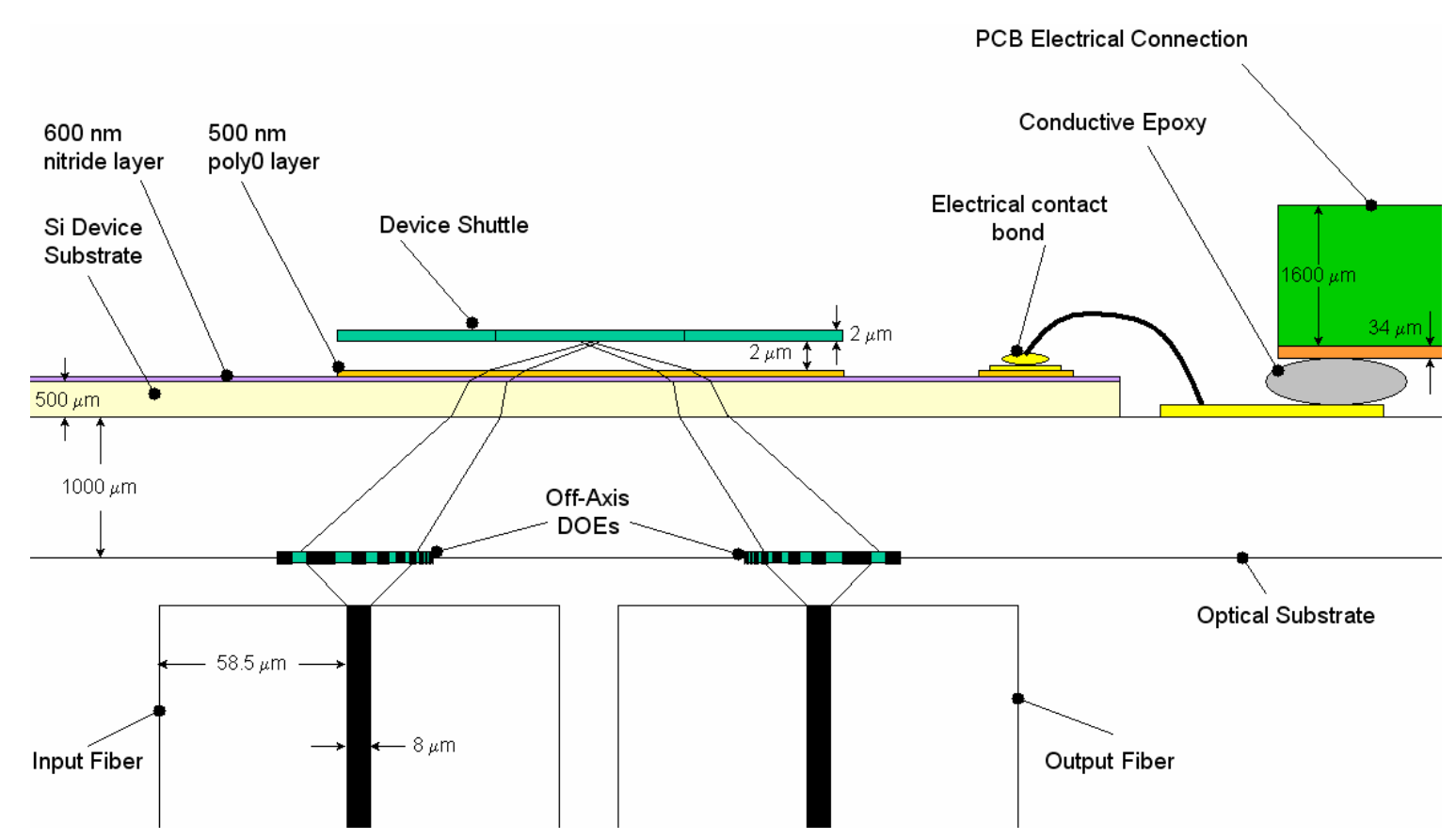

Figure 1.2: Proposed off-axis opto-electrical MEMS substrate.

A single fiber configuration is also possible that would also use a DOE to generate a probe beam with single or multiple focal points to the LCR diffraction grating. This configuration could be used to investigate out of plane motion of the device using laser vibrometry techniques in addition to the normal in-plane motion of a LCR. 


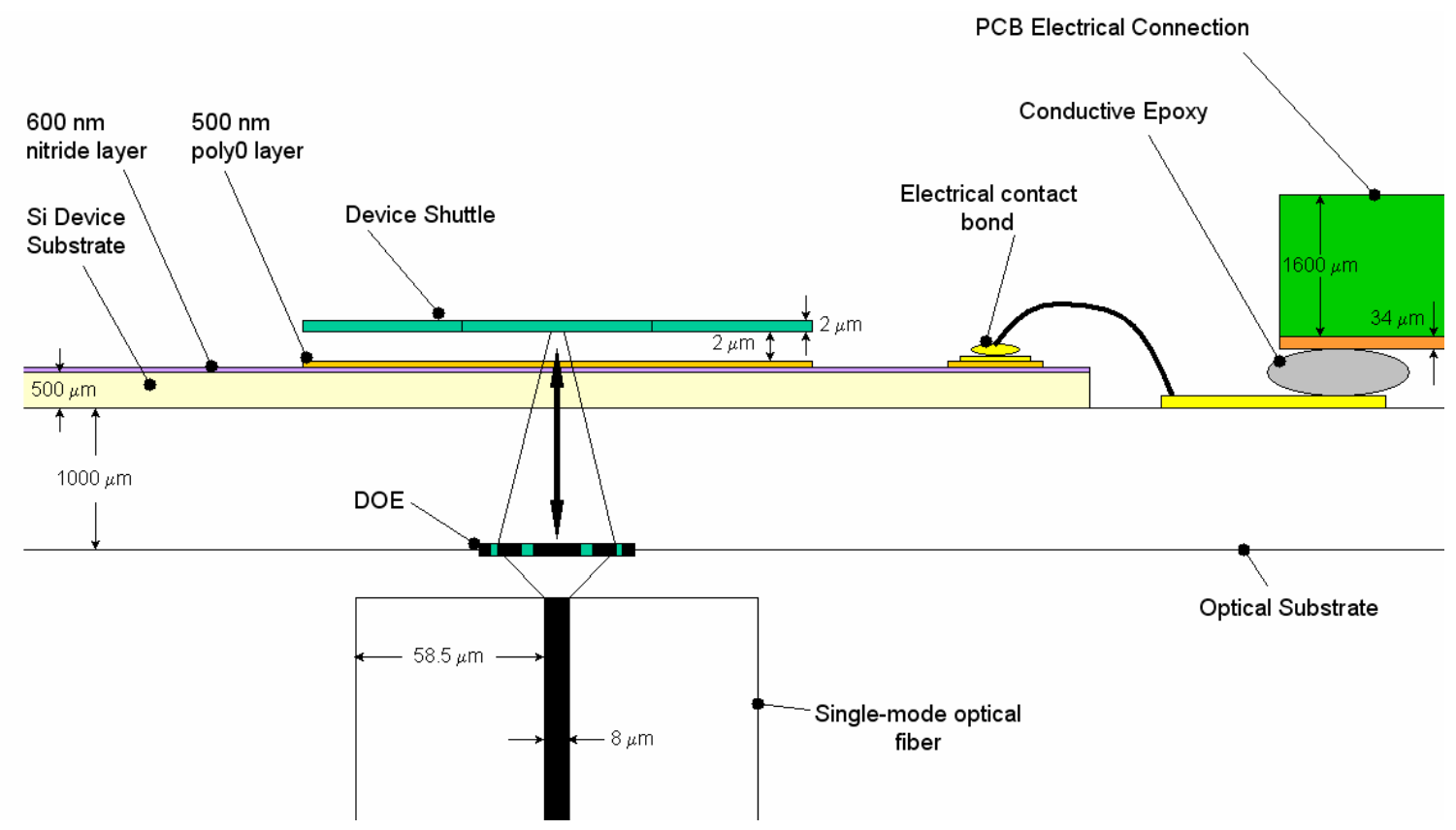

Figure 1.3: Proposed on-axis opto-electrical MEMS substrate.

The process to generate bondable $\mathrm{Cr} / \mathrm{Au}$ electrical traces on the optical substrate shown in the above figures has been successfully designed and implemented [16]. The completed opto-electrical substrate shown in Figure 1.4 demonstrates a more sophisticated method of delivering power to the die with 124 electrical connections compared to a maximum of approximately 36 with the current die package illustrated in Figure 1.5. The MEMS die will be located at the center of the package with DOEs etched on the backside of the optical substrate. 


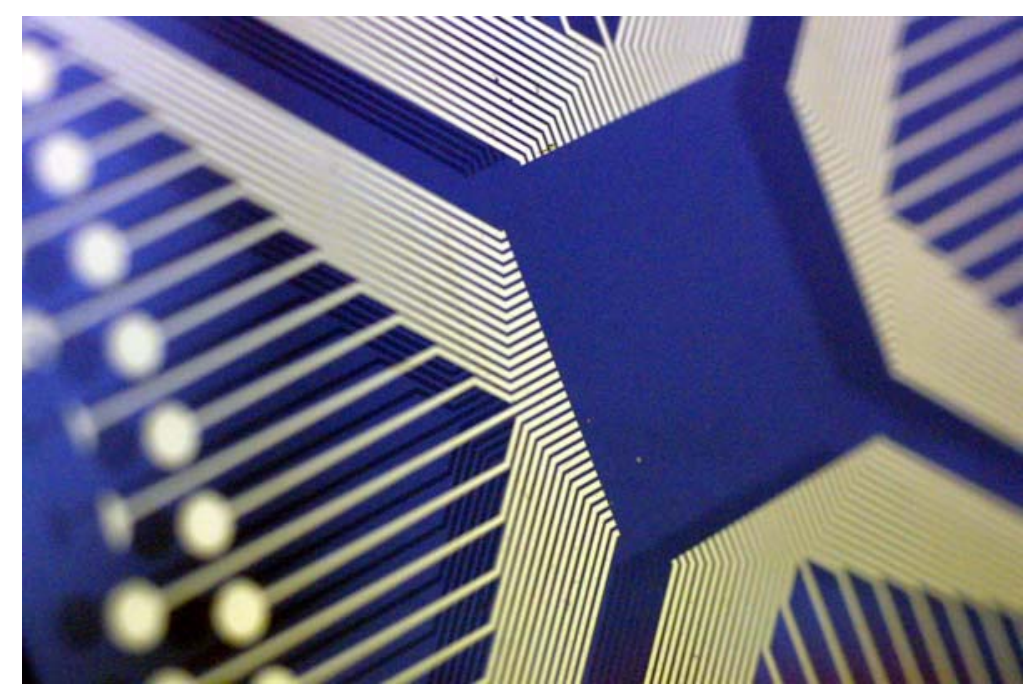

Figure 1.4: $\mathrm{Cr} / \mathrm{Au}$ electrical traces on current iteration of opto-electrical substrate [16].

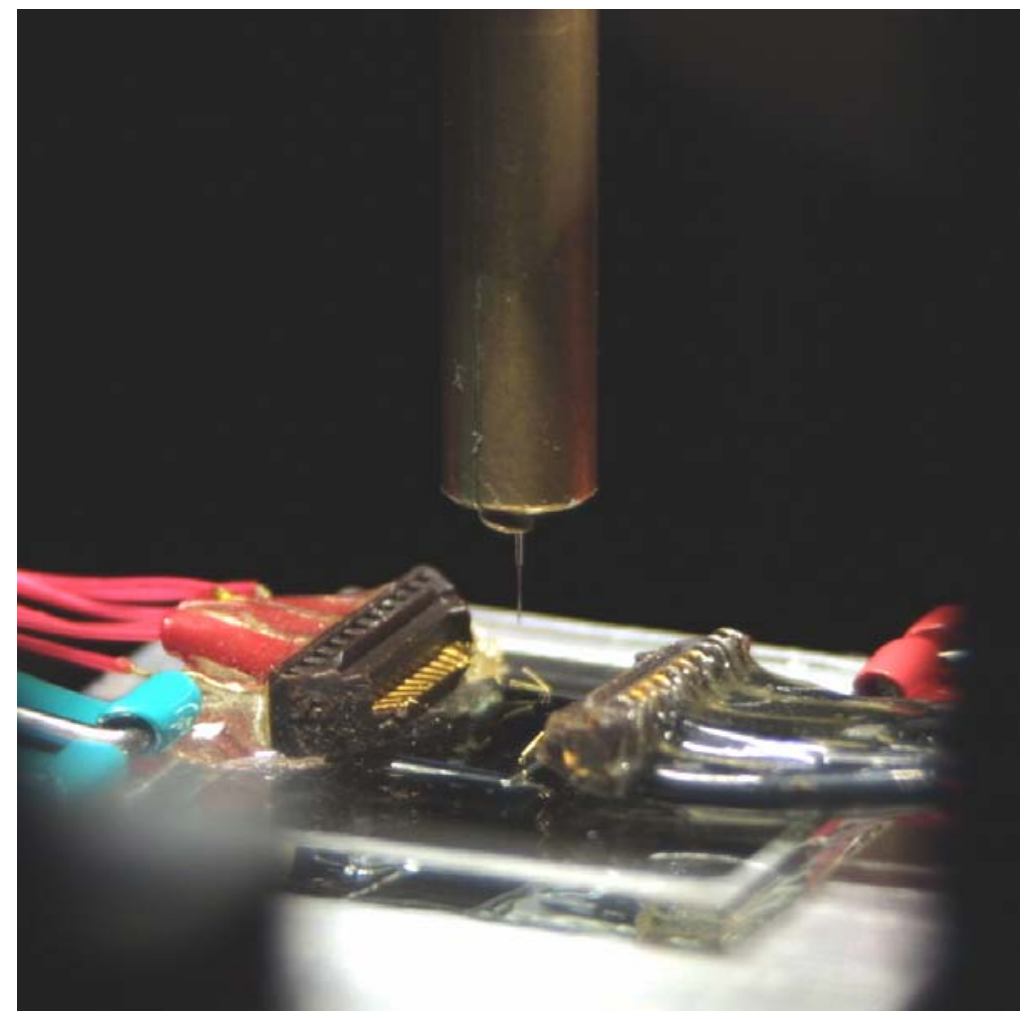

Figure 1.5: Current electrical packaging with optical probe fiber. 


\subsection{Diffractive Optical Element Design}

In order to achieve these free-space architectures for MEMS IOM, the modeling, design, fabrication and testing of DOEs is required. Establishing and experimentally validating such a process is the primary purpose of this work. Design of the DOEs will be performed using the scalar wave approximation to simulate desired input/output wave forms and a transfer function for propagation between the lens and focal plane. The wave forms and transfer function will be used to generate a lens profile through a simulated holographic technique that takes into account propagation distance to the focal plane as well as index of refraction of the lens material. The lenses will then be fabricated and optically tested to compare experimental results with simulated output.

In order to be usable in packaging configurations such as those shown in the figures above, the desired designed lens output will be single or multiple optical probe beams with beam diameters of $10 \mu \mathrm{m}$ located $1.5 \mathrm{~mm}$ or $5 \mathrm{~mm}$ from the lens and either on-axis or up to $300 \mu \mathrm{m}$ off-axis in relation to the normal of the lens. While continuous phase functions will be output from the simulation and design, binary phase DOEs will be fabricated to reduce fabrication complexity and achieve the desired basic tool and process validation.

\subsection{Organization of Thesis}

As motivated in this chapter, realization of an integrated optical probing system depends on effective DOEs that can guide and modify the probe beam resulting in useful positional information of device motion for fault detection and control over the lifetime of the device. Chapter 2 will give a brief theoretical explanation of ray optics, wave optics and electromagnetic optics before explaining the scalar wave theory of diffraction and interference used to create DOEs as well as discussing the propagation of the probe beam from the DOE through the substrate and MEMS die. Chapter 3 will discuss the implementation of the theory from Chapter 2 to generate the desired DOEs and simulate propagation of the diffracted beam through the MEMS structural layers. Conversion of the analog lens phase pattern to a binary pattern and then conversion to CIF file format for importation to CAD software for optical mask fabrication is also detailed. Chapter 4 will detail photolithography and reactive ion etch (RIE) procedures used for lens 
fabrication followed by comparisons of experimentally measured lens performance with simulation from Chapter 3. Chapter 5 will offer conclusions as well as suggestions for future research. 


\section{Chapter 2: Theory}

Chapter 2 discusses the theory that was used to generate and test diffractive optical elements (DOE). The study of optics can be divided into four areas that build on one another, beginning with the simplest description, ray optics, and consecutively more complex theories of wave optics, electromagnetic optics and finally quantum optics [17]. A brief overview will be given of ray optics, wave optics and electromagnetic optics with the primary focus on the scalar wave optic treatment of Gaussian beams and diffraction in isotropic, homogeneous, non-magnetic media which is the foundation of the work of this thesis.

\subsection{Ray Optics}

Ray or geometrical optics is the simplest model to describe the propagation of light and is useful primarily when the size of material structures and focal lengths are much larger than the wavelength of the light being observed. The ray optics model disregards the wave nature of light and treats the propagation as straight lines that interact with geometric shapes which modify the path of the ray depending on the material that is traversed. This interaction with different materials can be described by the refractive index or index of refraction of the material given by 


$$
n=\frac{c_{o}}{c},
$$

where $c_{o} \approx 3.0 \times 10^{8} \mathrm{~m} / \mathrm{s}$ is the velocity of light in free space and $c$ is the velocity of the light in the material. An example of how the refractive index affects the path of light can be seen in the propagation of a ray at the interface of two different materials such as air and quartz glass which have indices of refraction 1.0003 and 1.46 respectively.

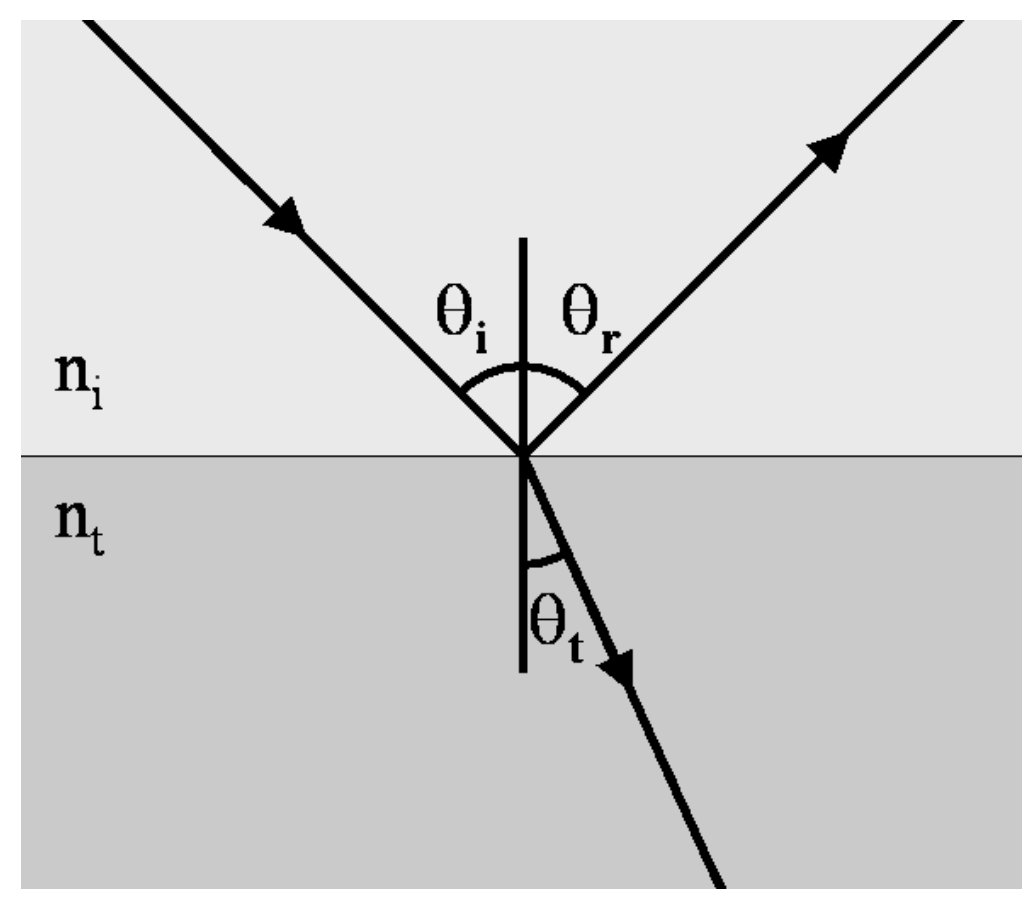

Figure 2.1: Example of Snell's Law.

Figure 2.1 illustrates the bending of the transmitted ray toward the normal as it passes through the material interface where $n_{t}$ represents a material that is a more optically dense than $n_{i}$ or $n_{i}<n_{t}$. The ray would bend away from the normal if $n_{i}>n_{t}$. These effects are described by the Law or Refraction or Snell's Law,

$$
n_{i} \sin \theta_{i}=n_{t} \sin \theta_{t}
$$

and the Law of Reflection,

$$
\theta_{i}=\theta_{r}
$$

where $\theta_{\mathrm{i}}, \theta_{\mathrm{r}}$ and $\theta_{\mathrm{t}}$ are the incident, reflected and transmitted angles of the ray. Specular reflection occurs when the wavelength is large compared to surface features, such as a mirror, and diffuse reflection occurs when the features are larger than the wavelength. 


\subsection{Wave Optics}

The geometric model is limited by the approximation of light as a ray and therefore cannot address phenomena that arise from the wave nature of light such as diffraction and interference. The scalar wave optics or physical optics model addresses these problems as well as the optical intensity, power and energy of a beam, different wavefront geometries, superposition of waves and dispersion in materials.

The wave model describes the field as it propagates using a position vector $\mathbf{r}$ and time $t$ with the complex wave function

$$
U(\mathbf{r}, t)=U(\mathbf{r}) \exp (j 2 \pi v t)
$$

where $U(\mathbf{r})=a(\mathbf{r}) \exp [j \varphi(\mathbf{r})]$ is the complex amplitude and $a(\mathbf{r}), \varphi(\mathbf{r})$ and $v$ are the amplitude, phase and frequency $(\mathrm{Hz})$ respectively. An assumption is made that the wave is monochromatic and sinusoidal, i.e. emitted from a source with only one frequency $v$. A laser is considered a quasimonochromatic source due to the narrow band of frequencies it emits and is the closest approximation of a true monochromatic source [18].

Different wavefront geometries, such as plane, spherical, and Gaussian can be described with the complex wave function but must satisfy the wave equation [17]

$$
\nabla^{2} U-\frac{1}{c^{2}} \frac{\partial^{2} U}{\partial t^{2}}=0
$$

where

$$
\nabla^{2}=\nabla \bullet \nabla \bullet()=\frac{\partial^{2}}{\partial x^{2}}+\frac{\partial^{2}}{\partial y^{2}}+\frac{\partial^{2}}{\partial z^{2}},
$$

is the divergence of the gradient or Laplacian and $\mathrm{c}$ is the velocity of the wave in the material being traversed. It is simpler to solve the wave equation for different phase fronts when you make the assumption of a sinusoidal profile, removing the time dependence after performing the second derivative with respect to time on the function. This results in the Helmholtz equation,

$$
\left(\nabla^{2}+k^{2}\right) U(\mathbf{r})=0
$$

where

$$
k=\frac{2 \pi v}{c}=\frac{\omega}{c}
$$


is the wavenumber, a phase constant of the propagating wavefront that is measured in radians/meter. The wavenumber is the magnitude of the vector $\mathbf{k}$ which is always normal to the propagation of the wave.

Another advantage of wave optics is the ability to calculate the optical intensity and amount of power contained in the wave. Optical intensity, the measure of optical power per unit area of the beam measured in watts $/ \mathrm{cm}^{2}$, is given by [17]

$$
I(\mathbf{r}, t)=2\left\langle u^{2}(\mathbf{r}, t)\right\rangle,
$$

where $\left\langle u^{2}(\mathbf{r}, t)\right\rangle$ is the time-average of the wavefunction. Assuming a monochromatic source and removing the time dependence, this can be redefined as

$$
I(\mathbf{r})=|U(\mathbf{r})| .
$$

Power is calculated by taking the integral of Equation 2.2-7 over the transverse area in question.

\section{Gaussian Beam}

The Gaussian solution of the wave and Helmholtz equations is of particular interest because it is the most suitable description of the output from a laser source $[19,20]$ and is represented by [17]

$$
U(\mathbf{r})=A_{0} \frac{W_{0}}{W(z)} \exp \left[\frac{-\rho^{2}}{W^{2}(z)}\right] \exp \left[-j k z-j k \frac{\rho^{2}}{2 R(z)}+j \varsigma(z)\right],
$$

with

$$
\begin{gathered}
W(z)=W_{0}\left[1+\left(z / z_{0}\right)^{2}\right]^{1 / 2}, \\
R(z)=z\left[1+\left(z_{0} / z\right)^{2}\right], \\
\varsigma(z)=\tan ^{-1} z / z_{0}, \\
W_{0}=\left(\frac{\lambda z_{0}}{\pi}\right)^{1 / 2},
\end{gathered}
$$

and 


$$
\rho^{2}=x^{2}+y^{2}
$$

$W_{0}$ is the waist radius of the beam at $z=0, W(z)$ is the radius of the beam as a function of distance $z, R(z)$ is the radius of curvature of the wavefront at a distance $z$ from the waist, $\varsigma(z)$ is the phase retardation of the beam for a given distance $z$ and $z_{0}$ is the Rayleigh range, the point where the beam area has doubled [17,21]. These parameters are illustrated in Figure 2.2.

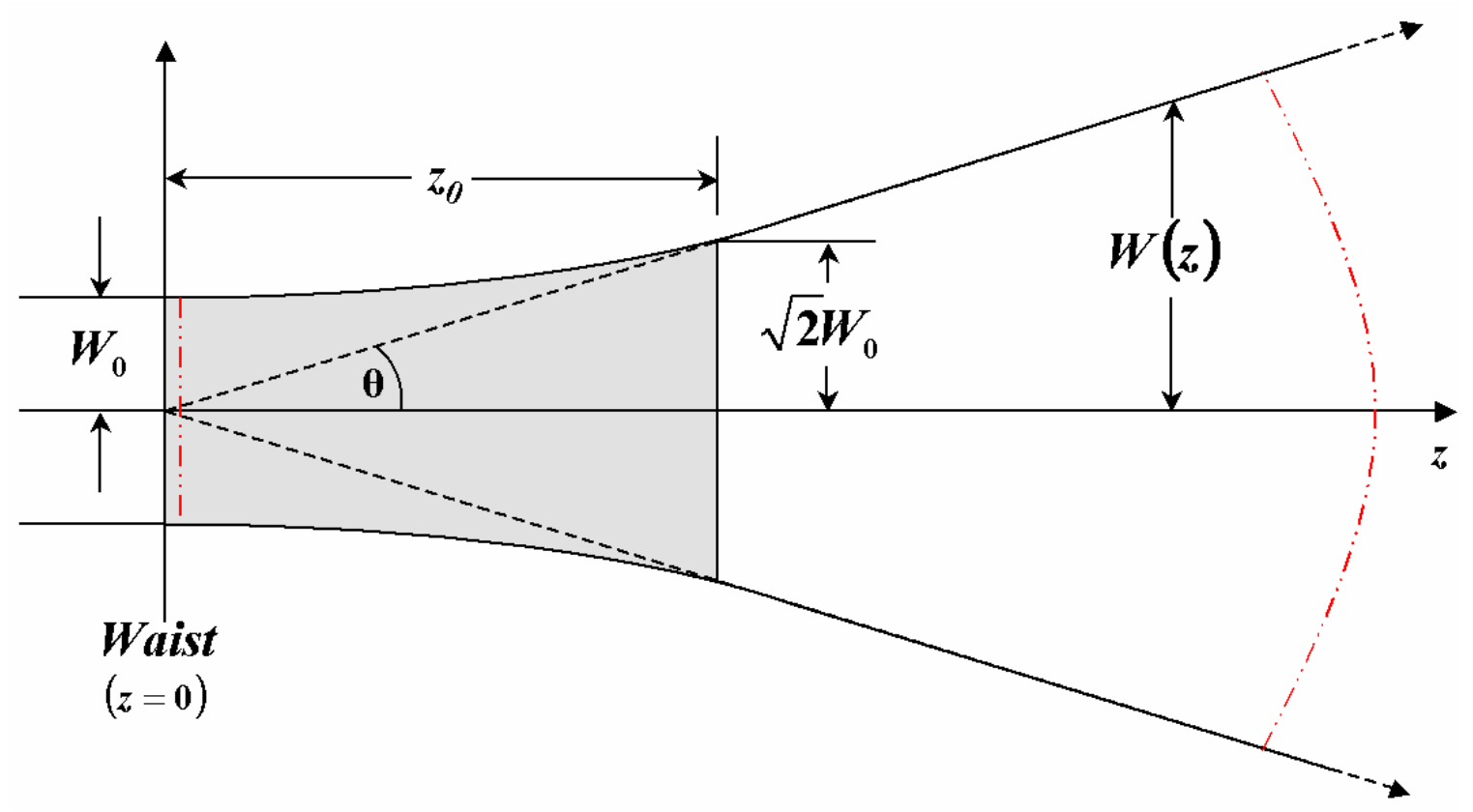

Figure 2.2: Gaussian Beam [21].

The dashed line at the waist illustrates the planar properties of the Gaussian beam while the curved dashed line represents a region far from the waist where the Gaussian beam begins to approximate a spherical wave. The phase of the beam again begins to approximate a planar wave as z approaches $\infty$.

\subsection{Electromagnetic Optics}

Although wave optics is more powerful than the ray optics model, it does not address all situations because it excludes the full vectoral electromagnetic information of the wave by only looking at one portion of either the electric or magnetic field at a time, 
creating issues when interface effects are of concern. The wave model is also limited by the approximation of paraxial propagation. Full vectoral analysis of the electromagnetic wave is based on the general form of Maxwell's equations [22,23], which are given in integral and differential form ,

$$
\begin{array}{cll}
\text { Faraday's Law } & \oint_{C} \bar{E} \bullet d \mathbf{l}=-\int_{S} \frac{\partial \bar{B}}{\partial t} \bullet d \mathbf{s}, & \nabla \times \bar{E}=-\frac{\partial \bar{B}}{\partial t}, \\
\text { Gauss's Law } & \int_{S} \bar{D} \bullet d \mathbf{s}=\int_{V} \tilde{\rho} d v, & \nabla \bullet \bar{D}=\widetilde{\rho}, \\
\text { Ampere's Law } & \oint_{C} \bar{H} \bullet d \mathbf{l}=\int_{S} \bar{J} \bullet d \mathbf{s}+\int_{S} \frac{\partial \bar{D}}{\partial t} \bullet d \mathbf{s}, & \nabla \times \bar{H}=\bar{J}+\frac{\partial \bar{D}}{\partial t}, \\
\begin{array}{l}
\text { Gauss's Law } \\
\text { (Magnetism) }
\end{array} & \oint \bar{B} \bullet d \mathbf{s}=0, & \nabla \bullet \bar{B}=0,
\end{array}
$$

where $\bar{E}$ is the electric field, $\bar{B}$ is the magnetic flux density, $\bar{D}$ is the electric flux density, $\tilde{\rho}$ is the volume charge density, $\bar{H}$ is the magnetic field and $\bar{J}$ is the current density.

The material's permittivity, $\varepsilon$, and permeability, $\mu$, play an important role in the complexity found in Maxwell's equations. The material composition is said to be homogeneous if the properties of the material are not dependent on position in the material. A material is considered isotropic if the electric susceptibility, $\chi_{\mathrm{e}}$, and magnetic susceptibility, $\chi_{\mathrm{m}}$, are independent of the orientation of $\bar{E}$ and $\bar{B}$ respectively. Electric and magnetic susceptibility are related to permittivity and permeability through

$$
\chi_{e}=\varepsilon_{r}-1
$$

and

$$
\chi_{m}=\mu_{r}-1,
$$

where $\varepsilon_{r}$ is the relative permittivity or dielectric constant and $\mu_{r}$ is the relative permeability. Further decomposition of $\chi_{\mathrm{e}}$ and $\chi_{\mathrm{m}}$ can be found in [22].

Linearity with regards to the electromagnetic field depends on the relationships of the electric field and the polarization, $\bar{P}$, of the material as well as the magnetic field and the magnetization, $\bar{M}$, of the material. Polarization of a material can be described as the summation of the dipole moments per unit volume and is a description of a dielectric 
material's ability to redistribute electrons in the presence of an external $\bar{E}$ field while magnetization of a material is the measure of the magnetic dipole moment per unit volume induced by an external $\bar{B}$ field $[17,22]$.. This can be seen using the following relationship between $\bar{E}, \bar{D}$ and $\bar{P}$

$$
\bar{D}=\varepsilon_{0} \bar{E}+\bar{P},
$$

and $\bar{B}, \bar{H}$ and $\bar{M}$

$$
\bar{B}=\mu_{0} \bar{H}+\mu_{0} \bar{M}
$$

where $\varepsilon_{0}$ and $\mu_{0}$ are the permittivity and permeability of free space respectively. Since we are dealing with nonmagnetic media, Equation 2.3-8 reduces to

$$
\bar{B}=\mu_{0} \bar{H} .
$$

If the medium that the electromagnetic field is propagating through is homogeneous, isotropic, linear, and nondispersive $\bar{E}, \bar{D}, \bar{H}$ and $\bar{B}$ are also related by the following equations,

$$
\bar{D}=\varepsilon_{0} \varepsilon_{r} \bar{E}=\varepsilon \bar{E},
$$

and

$$
\bar{B}=\mu_{0} \mu_{r} \bar{H}=\mu \bar{H},
$$

Substitution of equations 2.3-10 and 2.3-11 into Maxwell's equations and the assumption that the medium in question has no free electric charges or currents, i.e. is nonconductive or a dielectric, results in the simplification

$$
\begin{aligned}
& \nabla \times \bar{E}=-\mu \frac{\partial \bar{H}}{\partial t}, \\
& \nabla \bullet \bar{D}=0, \\
& \nabla \times \bar{H}=\varepsilon \frac{\partial \bar{E}}{\partial t}, \\
& \nabla \bullet \bar{B}=0,
\end{aligned}
$$

Assuming a sinusoidal time variation of the field quantities, Maxwell's equations can be written in time harmonic form as [22]

$$
\begin{aligned}
& \nabla \times \mathbf{E}=-j \omega \mu \mathbf{H}, \\
& \nabla \bullet \mathbf{D}=0,
\end{aligned}
$$




$$
\begin{aligned}
& \nabla \times \mathbf{H}=j \omega \varepsilon \mathbf{E}, \\
& \nabla \bullet \mathbf{B}=0 .
\end{aligned}
$$

These phasor forms can be manipulated into the wave equation for either the electric or magnetic field but have the advantage over wave optics of being applicable in the paraxial as well as non-paraxial region.

\subsection{Interference and Diffraction}

Coherence and frequency are the keys to interference. The discussion of interference and diffraction must be preceded by a better description of optical waves with respect to temporal and spatial coherence.

\section{Temporal Coherence}

Temporal coherence is measured in terms of coherence time $\Delta t_{c}$, the length of time where the phase of the wave is fairly constant [18,24], which corresponds to the coherence length,

$$
\Delta l=c \Delta t_{c}
$$

where $c$ is the velocity of the wave in the medium. The value of $\Delta t_{c}$ and the frequency bandwidth $\Delta v$ can be related by [24]

$$
\Delta v \approx \frac{1}{\Delta t_{c}}
$$

The ideal case of a fully coherent monochromatic source would have $\Delta t_{c}=\infty$ with the equivalent $\Delta v=0$ and $\Delta l=\infty$. A quasimonochromatic source, such as the laser, is a more realistic approximation of temporal coherence due to the small range of frequencies $\Delta v$ and relatively large $\Delta t_{c}$ and subsequent $\Delta l$. The wave is then considered coherent as long as $\Delta l$ is much larger than the distance from the source to the point of observation.

\section{Interference}

Interference can be either constructive or destructive and occurs when coherent or partially coherent waves that have a distinct phase relation to each other combine. The phase difference between waves can occur due to a difference in path length, reflection 
which causes a phase shift of $\pi$ when $n_{i}<n_{t}$ as in Figure 1.1 and thin films [25]. A stable interference pattern cannot form if the waves are temporally and spatially incoherent, i.e. $\Delta l$ is smaller than the distance from the source to the plane of interest, because the random phase variations in the resulting wave cancel. A stable interference pattern also cannot form if the frequencies of the waves are largely different. The effects of interference are addressed by the wave model. The principle of superposition applies to solutions of the wave equation.

\section{Diffraction}

Diffraction is the result of interference that occurs whenever a propagating wave, be it electromagnetic or a wave conducted in a medium such as gas or liquid, interacts with an aperture or edge. Optical diffraction describes the deviation of an electromagnetic wavefront from the path predicted by the geometric or ray optics model. This phenomena can generally be ignored as long as the wavelength of the wave is much smaller than the aperture it impinges upon but must be taken into account when the aperture size approaches the wavelength of the propagating wave [26,25,27]. Figure 2.3 [28] is an example of diffraction in water where the wavelength of the wave is readily apparent.

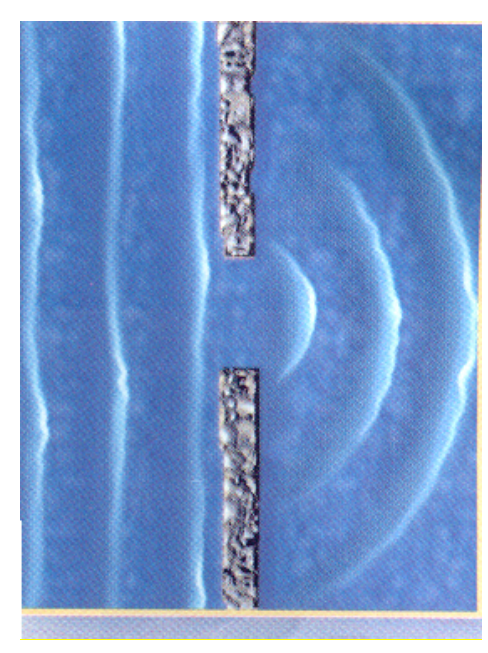

Figure 2.3: Water wave example of diffraction [28]. 
The scalar wave optical treatment of diffraction can be divided into Fresnel and Fraunhoffer approximations with the assumptions that the beam is paraxial with respect to the axis of propagation and that the structures of the DOE are large compared to the wavelength used. Both the Fresnel and Fraunhoffer approximations pertain to the transfer function that describes the propagation and evolution of the field as it travels along the optical axis.

The paraxial approximation, also known as the small angle approximation, is appropriate when the angle of incidence with respect to the optical axis is small and is valid when the following conditions are true,

$$
\operatorname{Sin}(\theta) \approx \theta, \operatorname{Tan}(\theta) \approx \theta .
$$

The graphs shown in Figure 2.4 are comparisons of the error versus angle when applying the paraxial approximation with the red line in Figure 2.4a denoting the point where the percent error between the value of $\operatorname{Tan}(\theta)$ and $\theta$ is $10 \%$ at approximately $30^{\circ}$. Figure $2.4 \mathrm{~b}$ reflects the region of operation of the off-axis lens designs fabricated for this research, with an angle of approximately $11.7^{\circ}$ corresponding to roughly $1.4 \%$ error.
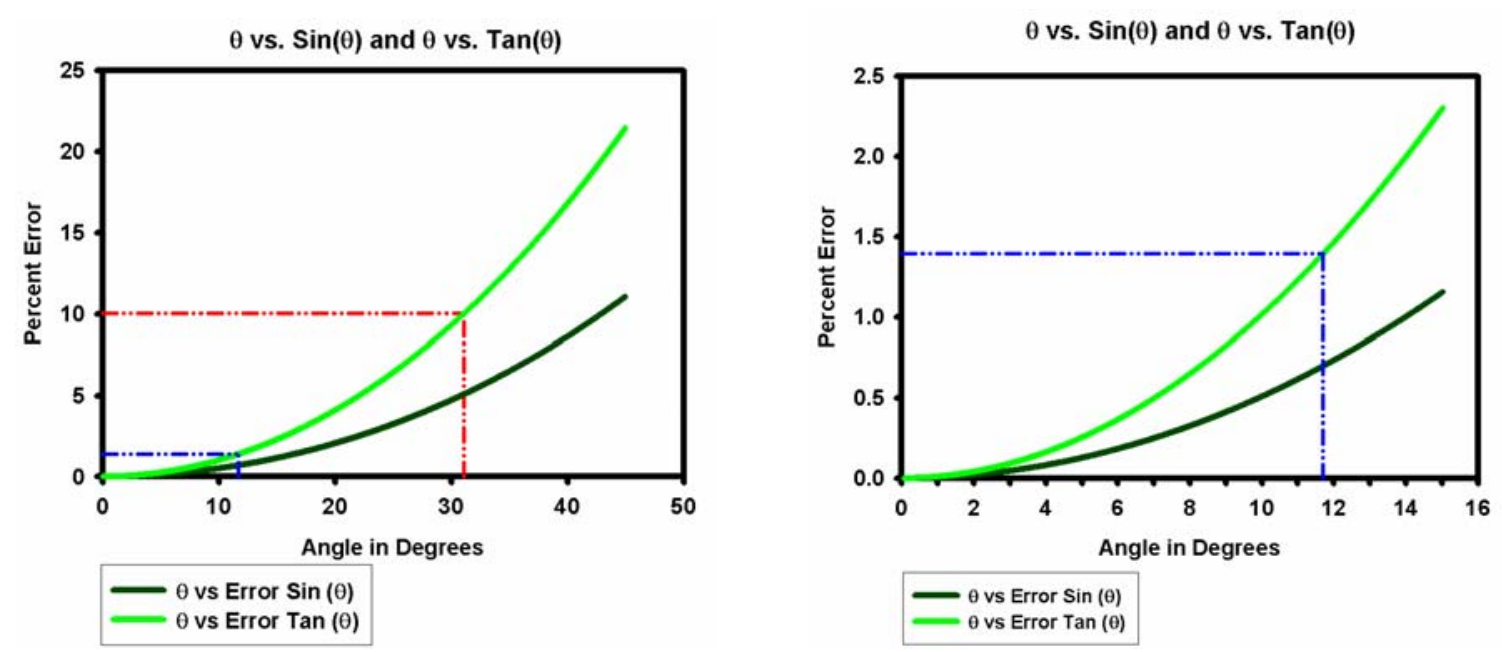

Figure 2.4: Validation of use of paraxial approximation.

Due to the distance between the DOE and the focal plane and the small angular displacement of the proposed monitoring technique, it will be possible to use scalar wave analysis and the Fresnel approximation or near field diffraction to calculate the DOE. 
The Fresnel approximation of diffraction is a simplification of the transfer function of free space and is applicable when the following condition is met [17]

$$
\frac{N_{\mathrm{F}} \theta_{m}^{2}}{4}<<1
$$

where the Fresnel number is

$$
N_{\mathrm{F}}=\frac{a^{2}}{\lambda d}
$$

and

$$
\theta_{m} \approx \frac{a}{d}
$$

where $a$ is the radial distance of the output plane and $d$ is the distance to the focal plane. This research will employ the impulse response of free space using this approximation and is given in Chapter 3.

\subsection{Material Interfaces}

The effects of material interfaces on the propagation of the wave must be addressed to ensure that the probe beam will be able to reach the test device with enough power to make meaningful measurements and enable a reasonable power budget for the receiver. Addressing this problem requires the calculation of the reflectance and transmittance at each interface and, in the off-axis case, the use of Snell's Law to determine the location of and the amount of power in the beam as it traverses the different layers of the proposed test structure.

The simplest case of reflectance and transmittance occurs at the boundary of two media with different indices of reflection $n_{1}$ and $n_{2}$ as shown in Figure 2.5. 


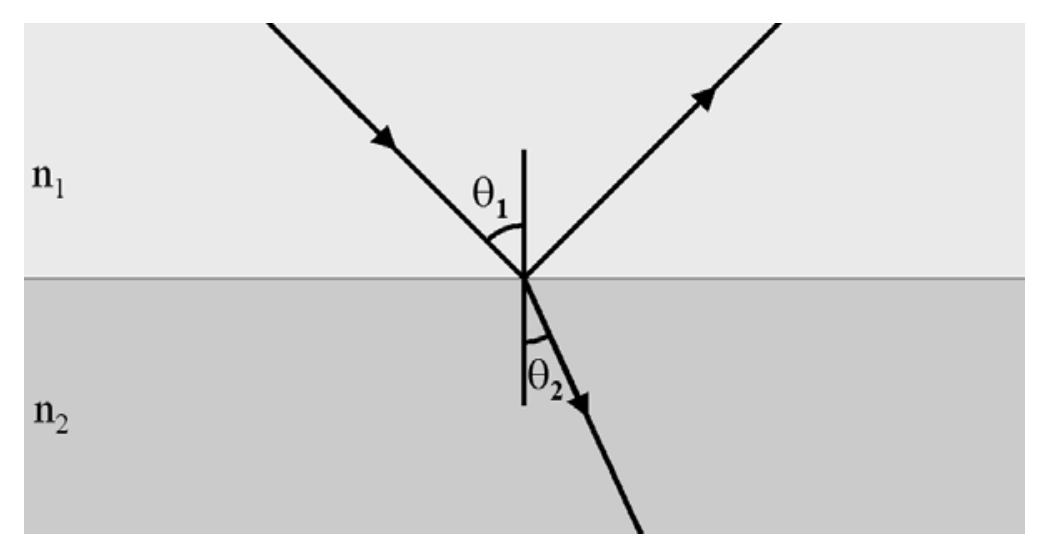

Figure 2.5: Reflected and transmitted beams at planar dielectric boundary.

The general Fresnel reflection and transmission coefficients $r$ and $t$ for a TE wave in a homogeneous nonmagnetic dielectric material can be calculated from [29]

$$
\begin{aligned}
& r_{12}=\frac{n_{1} \cos \theta_{1}-n_{2} \cos \theta_{2}}{n_{1} \cos \theta_{1}+n_{2} \cos \theta_{2}}=\frac{p_{1}-p_{2}}{p_{1}+p_{2}}, \\
& t_{12}=\frac{2 n_{1} \cos \theta_{1}}{n_{1} \cos \theta_{1}+n_{2} \cos \theta_{2}}=\frac{2 p_{1}}{p_{1}+p_{2}},
\end{aligned}
$$

with

$$
p=\sqrt{\frac{\mu \varepsilon}{\mu_{0} \varepsilon_{o}}} \cos \theta=\frac{c_{0}}{c} \cos \theta=n \cos \theta,
$$

where $p$ is also an example of the connection between geometric and electromagnetic optics in the form of the refractive index and the permittivity and permeability of the material. At normal incidence, Equations 2.5-1 and 2.5-2 reduce to

$$
r_{12}=\frac{n_{1}-n_{2}}{n_{1}+n_{2}}
$$

and

$$
t_{12}=\frac{2 n_{1}}{n_{1}+n_{2}} .
$$

These values can then be used to calculate the reflectivity $R$ and transmissivity $T$ using [29]

$$
R_{12}=\left|r_{12}\right|^{2}=r_{12} r_{12}^{*}
$$




$$
T_{12}=\frac{p_{2}}{p_{1}}\left|t_{12}\right|^{2}=\frac{p_{2}}{p_{1}} t_{12} t_{12}^{*}
$$

and

$$
R_{12}+T_{12}=1
$$

where * denotes the complex conjugate.

Fiber to Borofloat Lens Interface

Ideally, a quarter pitch graded index (GRIN) lens would be attached between the delivery fiber and the DOE with index matching optical epoxy. This would collimate the output of the fiber, reducing divergence and more closely match the simulation assumptions of a Gaussian input beam with the waist located at the DOE.

\section{Propagation Through Borofloat Substrate to Borofloat/Silicon Interface}

The transmissivity through the Borofloat and Si substrates can be calculated using the input angle into the Borofloat substrate for the off-axis DOE case, Snell's Law and Equation 2.5-7 neglecting secondary reflections from the interfaces.

\section{Propagation Through Silicon Wafer to MEMS Device}

A layer can be termed a thin film when the layer thickness is on the order of the freespace wavelength of the wave propagating in the medium. When dealing with thin films, it is possible to use a matrix method to condense multiple interfaces to one $2 \mathrm{x} 2$ characteristic matrix for the entire system. The generic form of the characteristic matrix for each thin nonmagnetic dielectric film with an incident TE wave is given as [29]

$$
\mathbf{M}=\left[\begin{array}{cc}
\cos \left(k_{0} n h \cos \theta\right) & \frac{-i}{p} \sin \left(k_{0} n h \cos \theta\right) \\
-i p \sin \left(k_{0} n h \cos \theta\right) & \cos \left(k_{0} n h \cos \theta\right)
\end{array}\right]=\left[\begin{array}{cc}
\cos \beta & \frac{-i}{p} \sin \beta \\
-i p \sin \beta & \cos \beta
\end{array}\right]
$$

with

$$
\beta=\frac{2 \pi n_{f i l m} h \cos \theta_{\text {film }}}{\lambda_{0}}
$$

and

$$
p=n_{\text {film }} \cos \theta_{\text {film }}
$$


where $h$ is the film thickness. In the case of normal incidence or within the region where the paraxial approximation is valid, Equation 2.5-9 simplifies to

$$
\mathbf{M}=\left[\begin{array}{cc}
\cos \beta & \frac{-i}{n_{f i l m}} \sin \beta \\
-i n_{\text {film }} \sin \beta & \cos \beta
\end{array}\right]
$$

and

$$
\beta=\frac{2 \pi n_{\text {film }} h}{\lambda_{0}} .
$$

Reflectivity and transmissivity are still calculated using the Fresnel coefficients with the matrix method but are simplified by using the values of the characteristic matrix instead of calculating at every interface. If the components of the characteristic matrix are given as

$$
\mathbf{M}=\left[\begin{array}{ll}
m_{11} & m_{12} \\
m_{21} & m_{22}
\end{array}\right],
$$

then the Fresnel coefficients become [29]

$$
r=\frac{\left(m_{11}+m_{12} p_{\mathrm{L}}\right) p_{1}-\left(m_{21}+m_{22} p_{\mathrm{L}}\right)}{\left(m_{11}+m_{12} p_{\mathrm{L}}\right) p_{1}+\left(m_{21}+m_{22} p_{\mathrm{L}}\right)}
$$

and

$$
t=\frac{2 p_{1}}{\left(m_{11}+m_{12} p_{\mathrm{L}}\right)+\left(m_{21}+m_{22} p_{\mathrm{L}}\right)}
$$

where $p_{1}$ and $p_{\mathrm{L}}$ are calculated using the first and last semi-infinite layers surrounding thin layers.

Figure 2.6 is a representation of semi-infinite layers, silicon and poly1, and thin film layers, designated $\mathrm{M}_{2}, \mathrm{M}_{3}$ and $\mathrm{M}_{4}$, contained in the proposed packaging system mentioned in Chapter 1. The silicon layer is considered semi-infinite due to the thickness of $500 \mu \mathrm{m}$ while the Poly1 layer will be assumed as semi-infinite and fully reflective with the output angle $\theta_{\mathrm{L}}$ only used for calculation of the thin layer transmission. The transmissivity of the Borofloat and Si layers must be calculated using Equations 2.5-6 and 2.5-7 


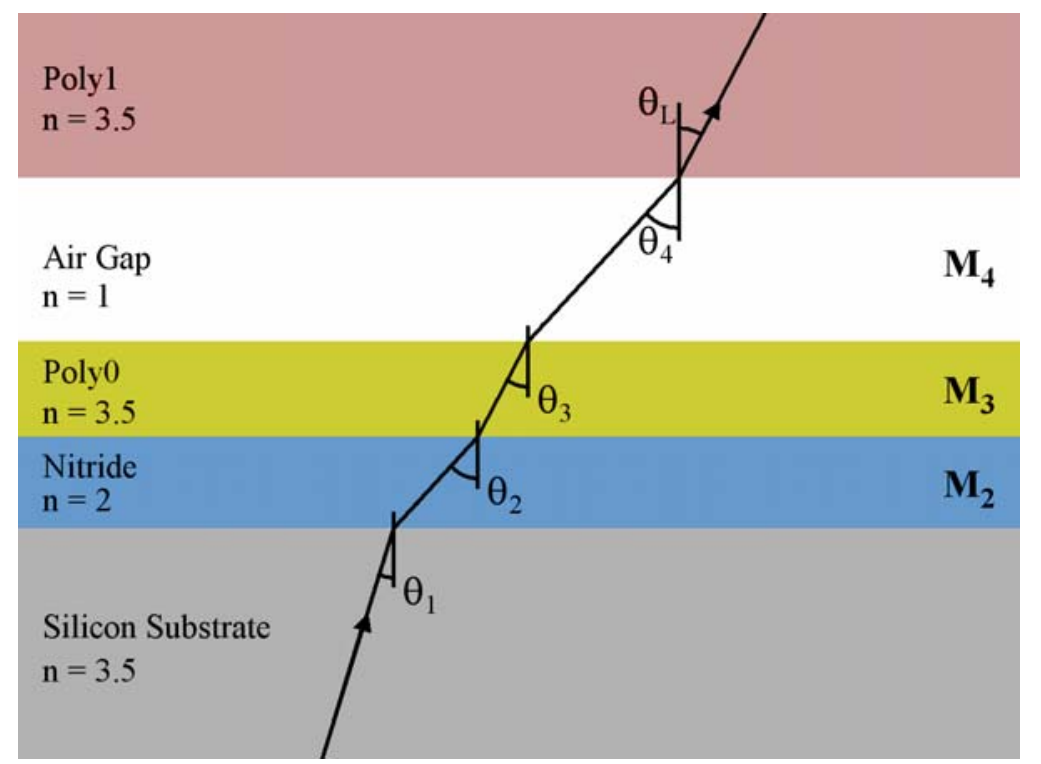

Figure 2.6: Transmittance through the thin films and semi-infinite layers of current MEMS LCR

The characteristic equation for this system would be

$$
\mathbf{M}=\left[\begin{array}{cc}
\cos \beta_{4} & \frac{-i}{p_{4}} \sin \beta_{4} \\
-i p_{4} \sin \beta_{4} & \cos \beta_{4}
\end{array}\right] \bullet\left[\begin{array}{cc}
\cos \beta_{3} & \frac{-i}{p_{3}} \sin \beta_{3} \\
-i p_{3} \sin \beta_{3} & \cos \beta_{3}
\end{array}\right] \bullet\left[\begin{array}{cc}
\cos \beta_{2} & \frac{-i}{p_{2}} \sin \beta_{2} \\
-i p_{2} \sin \beta_{2} & \cos \beta_{2}
\end{array}\right]
$$

or

$$
\mathbf{M}=\mathbf{M}_{4} \mathbf{M}_{3} \mathbf{M}_{2},
$$

with

$$
p_{1}=n_{\mathrm{Si}} \cos \theta_{\mathrm{Si}}
$$

and

$$
p_{\mathrm{L}}=n_{\mathrm{P} 1} \cos \theta_{\mathrm{P} 1} .
$$

The equivalent layout is shown in Figure 2.7. 


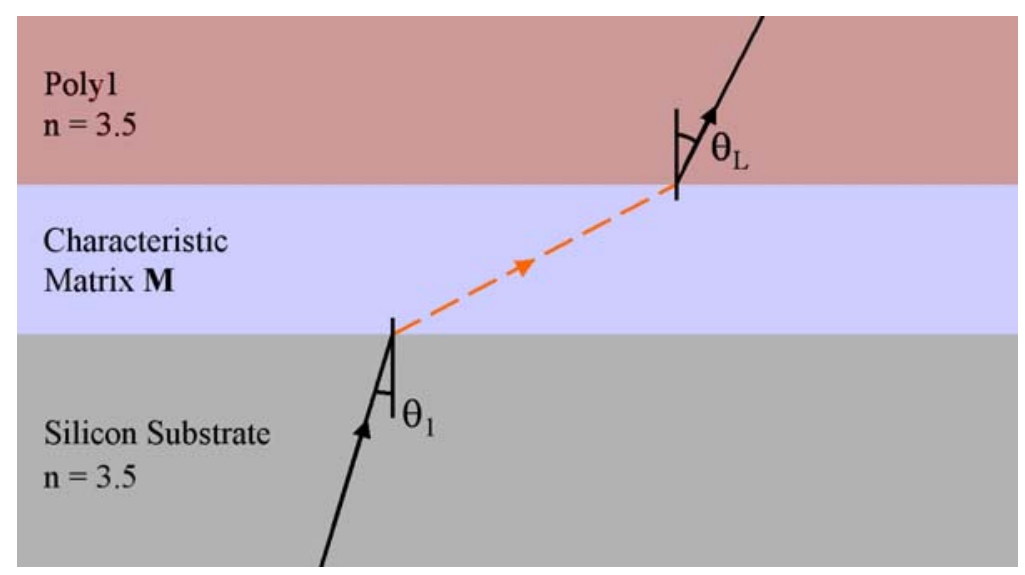

Figure 2.7: Equivalent thin film and semi-infinite layers by using characteristic matrix.

Using Equations 2.5-17 through 2.5-20, the transmissivity of the probe beam through the thin layers of the system from the Si substrate to the Poly1 layer is found to be approximately $77 \%$.

\section{Propagation of Reflection from Device to Second Fiber/Lens Interface}

The transmissivity of the return path can also be calculated using Equations 2.5-17 through 2.5-20 by reversing the order of the matrix,

$$
\mathbf{M}=\mathbf{M}_{2} \mathbf{M}_{3} \mathbf{M}_{4},
$$

also resulting in a transmissivity of approximately $77 \%$ through the thin layers from Poly1 to the Si interface. The duplication of the transmissivity can be explained by the symmetry of the system which will also be duplicated for the Si and Borofloat layers. 


\section{Chapter 3: Simulation}

Generation of the lens profiles was accomplished by simulating a holographic system consisting of an input beam, output beam and transfer function. Sub-functions were written in MATLAB to construct arrays for the input/output beams and the transfer function based on parameters such as beam radius and desired focal length. Additional functions were written to convert and save the lens profiles in a format that would be easily imported into a CAD software package such as L-Edit. These programs can be found in Appendix A and Appendix B located after chapter 5.

\subsection{Lens Generation Method}

CreateLens. $m$ and TestLens. $m$ are the main programs written in MATLAB where the user can define a range of values such as index of refraction of the lens material, wavelength of the beams and focal distance which are passed to the various sub-functions to generate and then test the lens profile. Sub-functions were employed to simplify coding, modification and debugging of the simulations. Memory constraints arising from the large size of the arrays generated, typically $1600 \times 1600$ or 2,560,000 elements, and memory constraints of MATLAB and Windows XP necessitated saving information in the form of arrays at the end of each sub-function to properly generate and test the DOEs. CreateLens.m and TestLens.m flowcharts of are given in Figures 3.1 and 3.2. 
Calc. Transfer Function

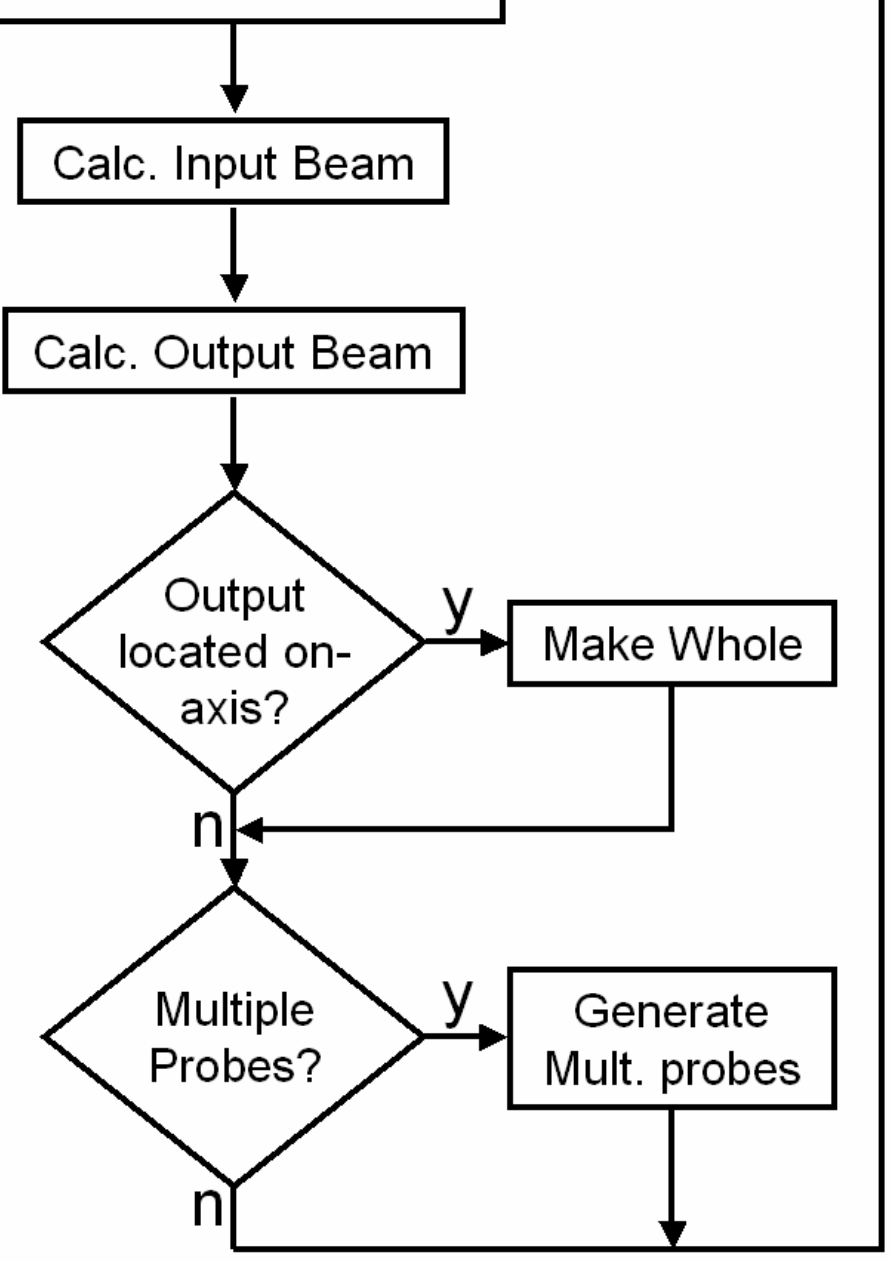

Calc. Aperture Shadow

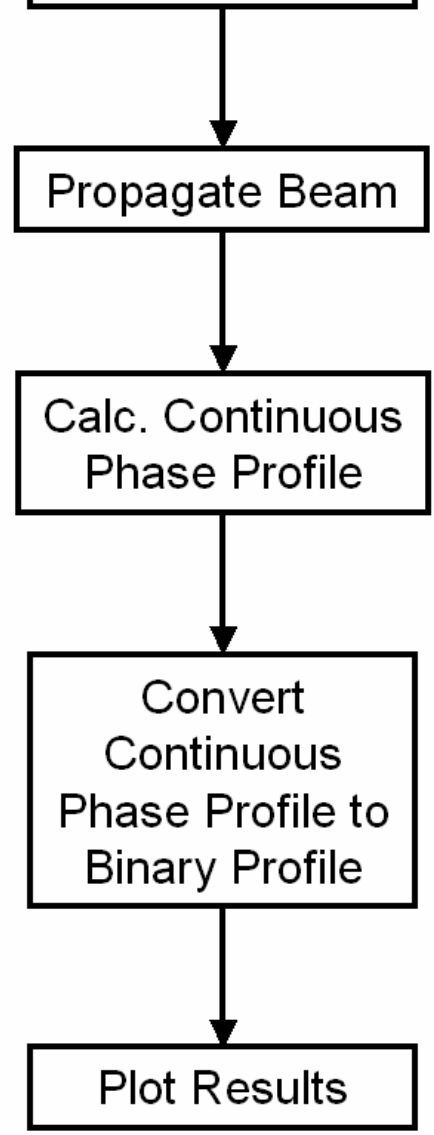

Figure 3.1: CreateLens.m flowchart. 


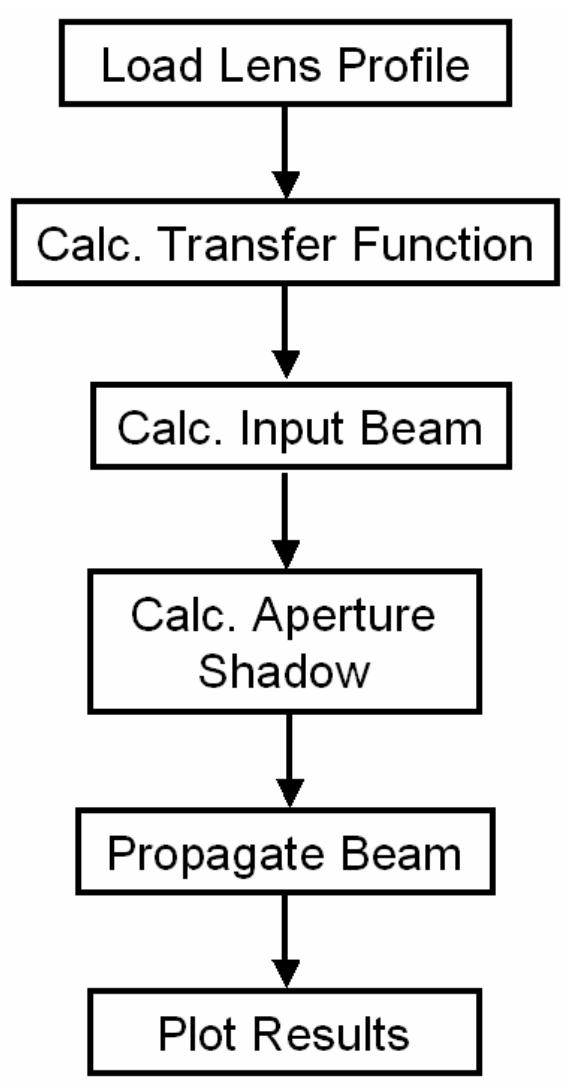

Figure 3.2: TestLens.m flowchart.

\subsubsection{Gaussian Beam Input/Output}

The MATLAB functions for beam generation are InBeam.m and either OutBeamQ.m or OutBeamNonCenter.m. The input/output beams are assumed to be Gaussian with different parameters that can be changed by the user in the main programs CreatLens.m and TestLens.m. The reader is referred to Equations 2.2-8 to 2.2-13 describing the scalar wave equations describing Gaussian beams located in Chapter 2 .

InBeam.m generates the desired input beam for the system. OutBeamQ.m was used to generate the desired output beam when the output was going to be centered in the focal plane by calculating only one quarter of the wave. This quarter wave array was saved and then called by MakeWholeQ.m which took advantage of the symmetry of the field to construct the full wave by matrix manipulation, thus reducing computation time.

OutBeamNonCenter.m was used if the output beam was not going to be centered in the focal plane of the system and MakeWholeQ.m was not applicable due to nonsymmetry of the field. This resulted in the calculation of the entire output plane which 
increased computation time from approximately 5 minutes to 2.5 hours for the output beam alone. The main cause of this increase was due to the method of memory allocation of arrays in MATLAB.

It was found that programs would operate much faster if array sizes were predetermined and defined prior to calculations because system memory necessary for the arrays was already set aside. This allowed MATLAB to fill positions in the array with calculated values instead of constantly resizing the array each time a new value was added. The large increase in calculation time when using OutBeamNonCenter.m was most likely attributed to the change from real numbers to complex numbers as the field went from zero values to non-zero complex values causing a doubling of memory requirements. As an example, a 3x3 double array of real numbers requires 72 bytes of memory compared to 144 bytes needed for a 3x3 double array of complex numbers. Initializing the array as complex or filling the array with complex numbers prior to substitution by calculated values seemed to have no effect as the array appeared to revert to real values until a complex number was entered. Figure 3.3 is an example of generated input and output beams.

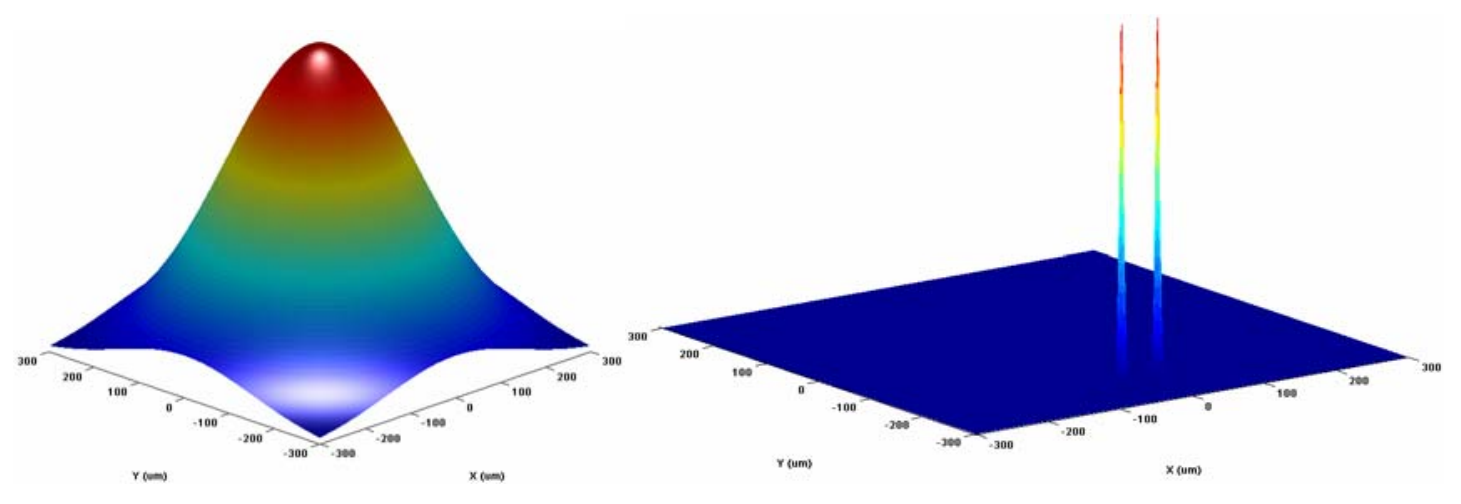

Figure 3.3: Generated input and output beams.

\subsubsection{General Transfer Function of Free space}

A mathematical function that describes the relationship between the input and output of a system is called a transfer function and, in this case, describes the different ways that a wavefront evolves as it propagates through free space from the lens to the output plane. The Fresnel approximation of the Impulse-Response Function of Free Space (FAIRFF) was chosen as the transfer function to simulate the propagation of the wavefront between 
the aperture shadow of the lens and the focal plane located a distance $d$ defined by the user. The FAIRFF is defined as [17]

$$
h(x, y) \approx h_{0} \exp \left[-j k \frac{\rho^{2}}{2 d}\right],
$$

with

$$
h_{0}=\left(\frac{j}{\lambda d}\right) \exp (-j k d),
$$

where $k$ is the wavenumber, $d$ is the distance traveled corresponding to the desired focal length of the lens and $\rho^{2}=x^{2}+y^{2}$. hhxycalc.m is the sub-function that calculates the transfer function based on the desired focal length and the size of the lens. Figure 3.4 is a representation of the desired use of the FAIRFF.

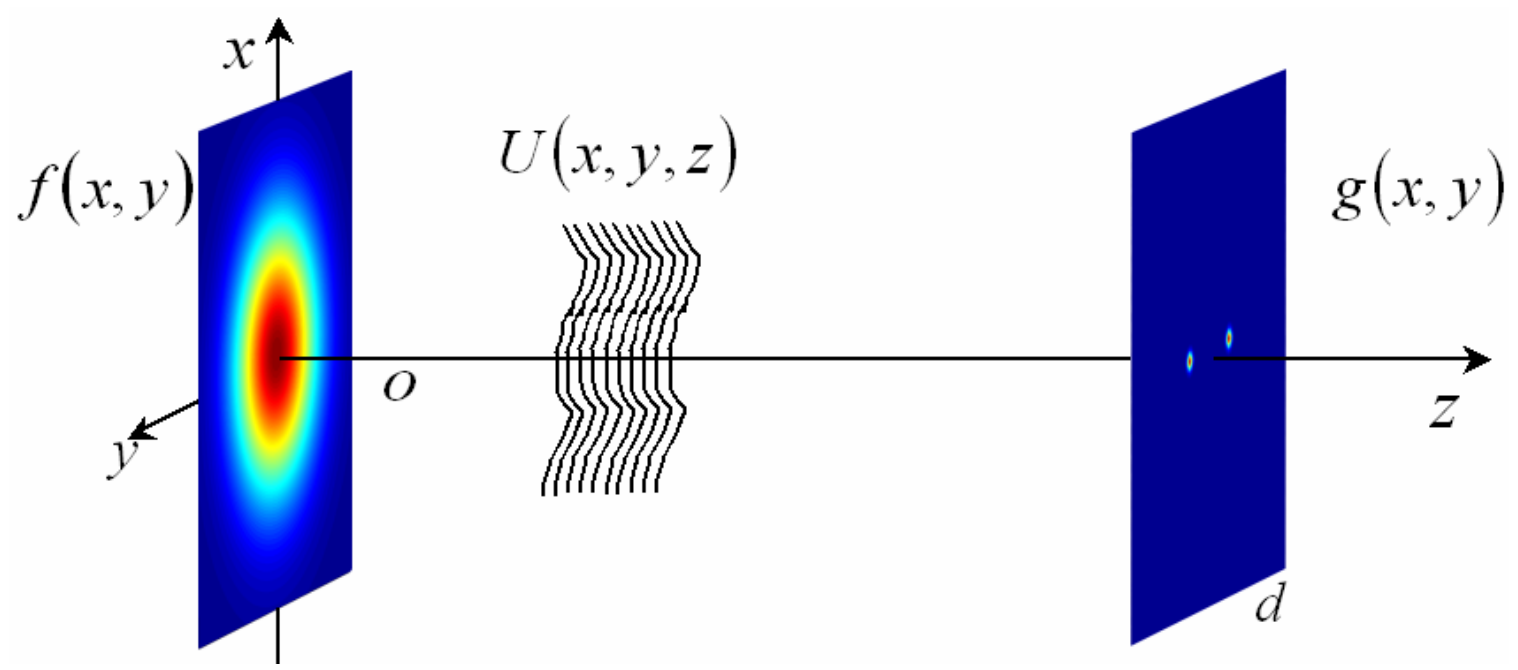

Figure 3.4: Transfer Function.

\subsubsection{Aperture Shadow}

The aperture shadow is the electromagnetic field distribution located immediately after the lens. PAS.m calculates the field by converting both the generated output beam and the transfer function to the frequency domain from the spatial domain using a Fast Fourier Transform (FFT). The output beam is then divided by the transfer function. The resulting array simulates the propagation of the desired output from the focal plane to the lens and is converted back to the spatial domain via Inverse Fast Fourier Transform (IFFT). 


\subsubsection{Lens Profile}

The aperture shadow and input beam arrays are used to calculate the transmittance array of the lens using the following relation for the complex amplitude transmission function [17]

$$
t(x, y)=\frac{U(x, y, z)}{U(x, y, 0)},
$$

where $U(x, y, z)$ and $U(x, y, 0)$ represent the aperture shadow and input beam respectively.

Conversion of the transmittance array to a lens profile is just a matter of solving the complex amplitude transmittance function of a transparent plate [17] for the thickness d as shown in Equation

$$
d=j \frac{t(x, y)}{n k_{o}} .
$$

This formalization of the transmittance function assumes a beam that satisfies the paraxial approximation in relation to the normal of the lens.

\subsubsection{Conversion from Continuous to Binary}

The lens profile that is generated resembles a continuous function due to the large number of sampling points (approximately $2 \times 10^{6}$ depending on lens size). It was decided to convert this continuous profile to a binary state for lens fabrication to reduce cost and complexity with the option of multi-level design in the future. The conversion to a binary lens was performed the function CalcBinary.m and calculating the wavelength of the incoming beam for the material of the lens, i.e. Borofloat, and using this value as a cutoff point. In the binary case this cutoff was.... Figure 3.5 is an example of a continuous profile and Figure 3.6 is the binary profile. 


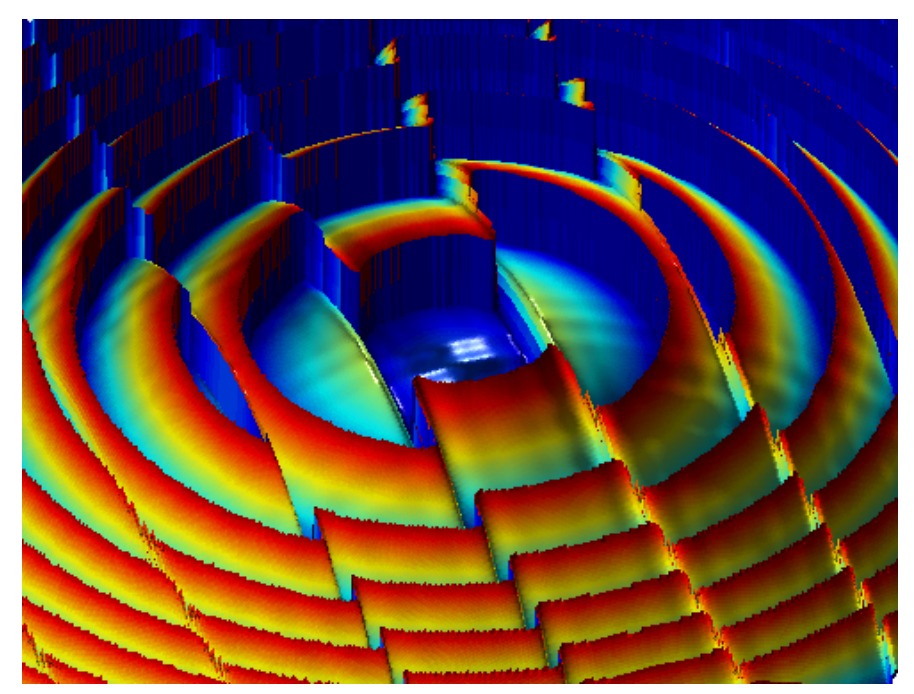

Figure 3.5: Central region of dual spot on-axis continuous profile.

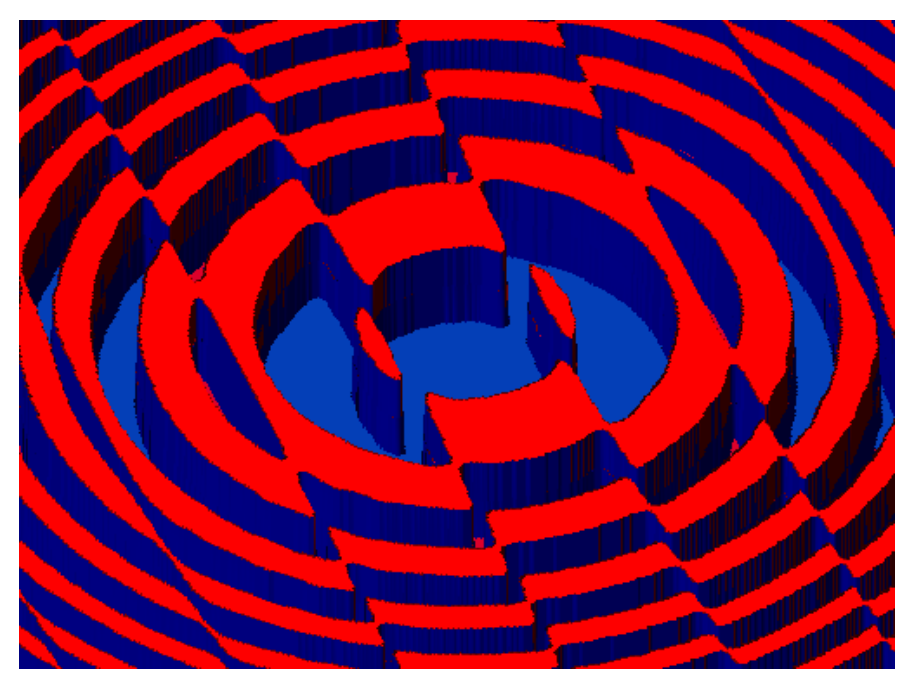

Figure 3.6: Central region of dual spot on-axis binary profile.

\subsection{On-Axis and Off-Axis DOE Generation and Simulation}

\subsubsection{On-axis}

Generation of an on-axis DOE assumes the input and output beams will be on-axis and normal to the plane of the DOE with the output spot size chosen as 10 um with focal lengths of $1.5 \mathrm{~mm}$ based on the geometry of the proposed package and $5 \mathrm{~mm}$ for ease of testing. It was found that the waist size of the input beam could be any size assuming the 
waist was located at the central portion of the lens plane for all cases including off-axis and multiple spot. This is possible because the Gaussian beam approximates a plane wave at this point and with the same phase information across the plane. Calculation of only a quarter of the output Gaussian was needed to describe the beam due to symmetry. Figure 3.7 is the normalized intensity plot of the input beam, Figure 3.8 is the normalized plot of a single probe output and Figure 3.9 is the resulting binary lens profile.
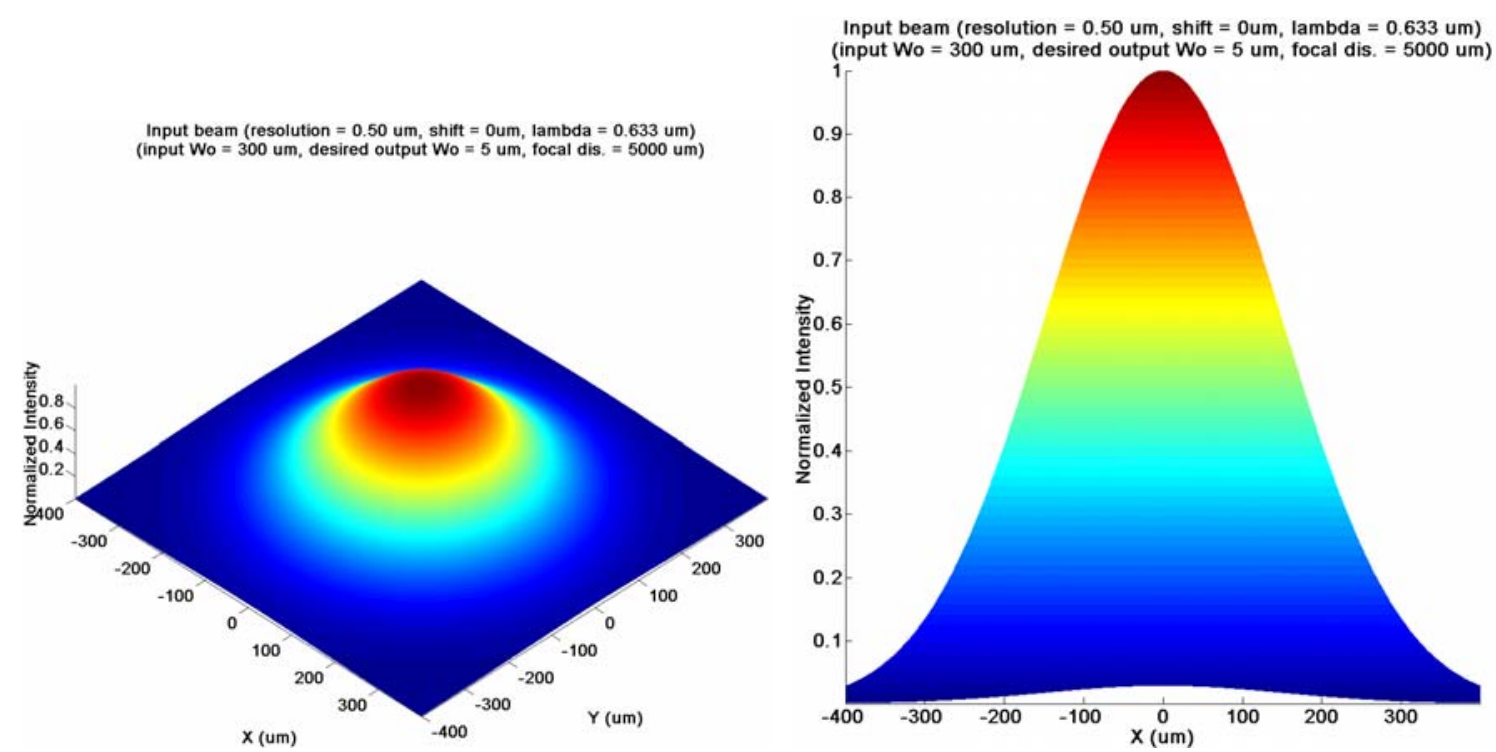

Figure 3.7: Normalized intensity plots of input Gaussian beam used for DOE generation. 

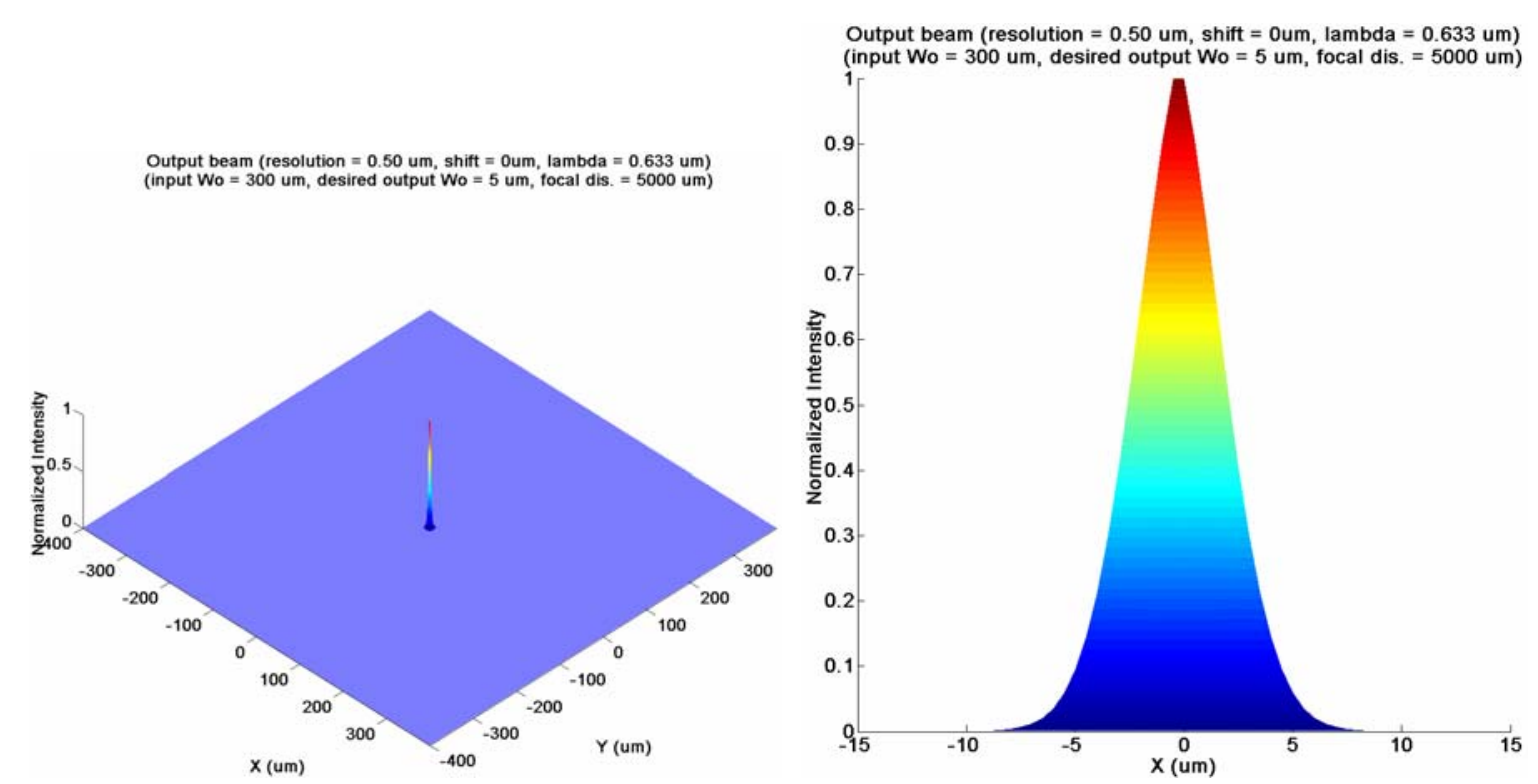

Figure 3.8: Normalized intensity plot of desired on-axis $10 \mu \mathrm{m}$ diameter Gaussian probe beam.
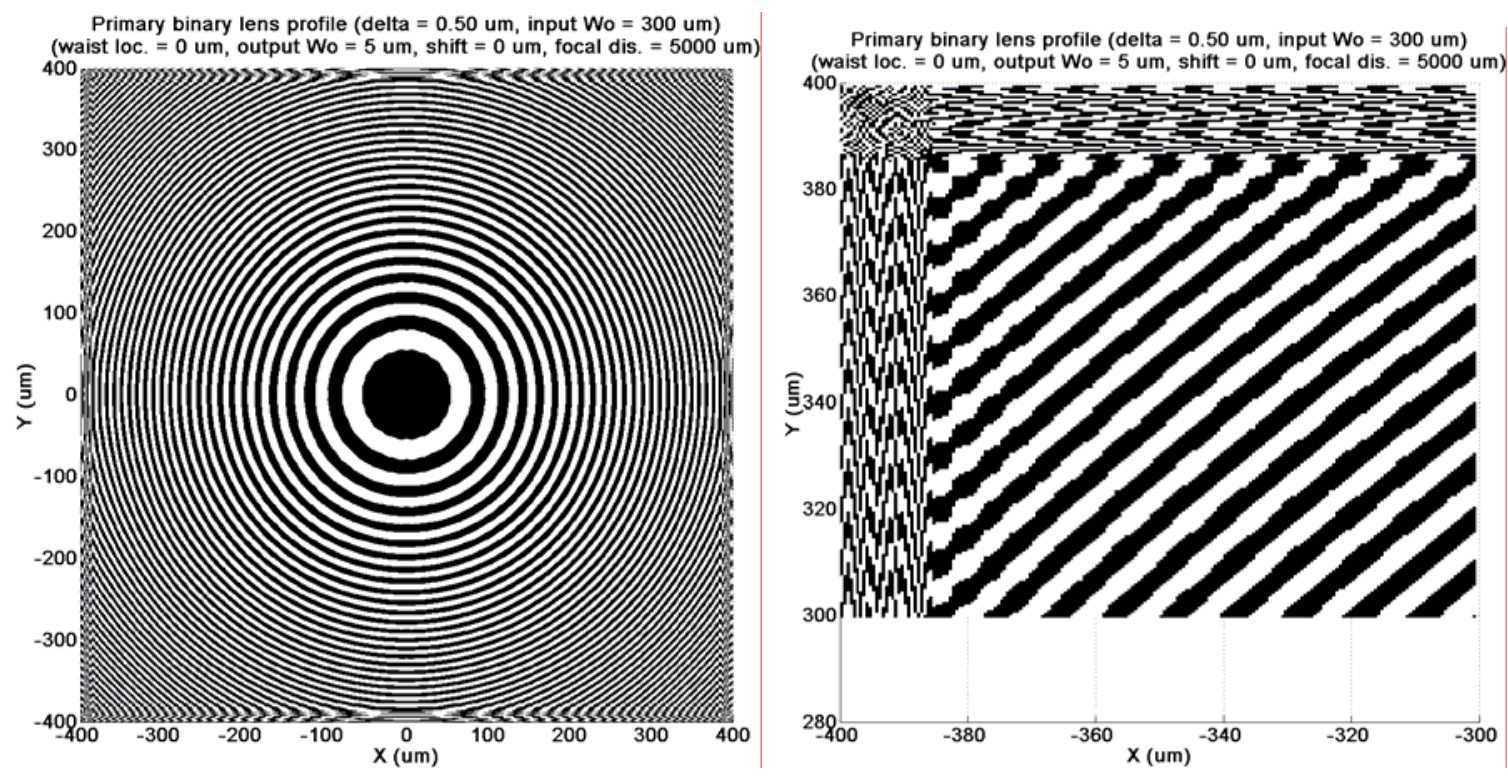

Figure 3.9: Generated single spot on-axis binary DOE.

The pixilated regions on the outer edges of the lens profile are assumed to be the result of the artifacts from the $0.5 \mu \mathrm{m}$ grid used for calculation of the fields and resulting lens. 


\subsubsection{Off-axis}

Off-axis simulation required the output of the system be shifted away from the central axis of propagation and was accomplished by calculating the entire output Gaussian beam with the ability to place the waist location anywhere in within the bounds of the output plane. The input beam calculations and focal lengths were also chosen as stated in Section 3.2.1. Figure 3.10 is the normalized intensity of a single off-axis output beam and Figure 3.11 is the resulting DOE.

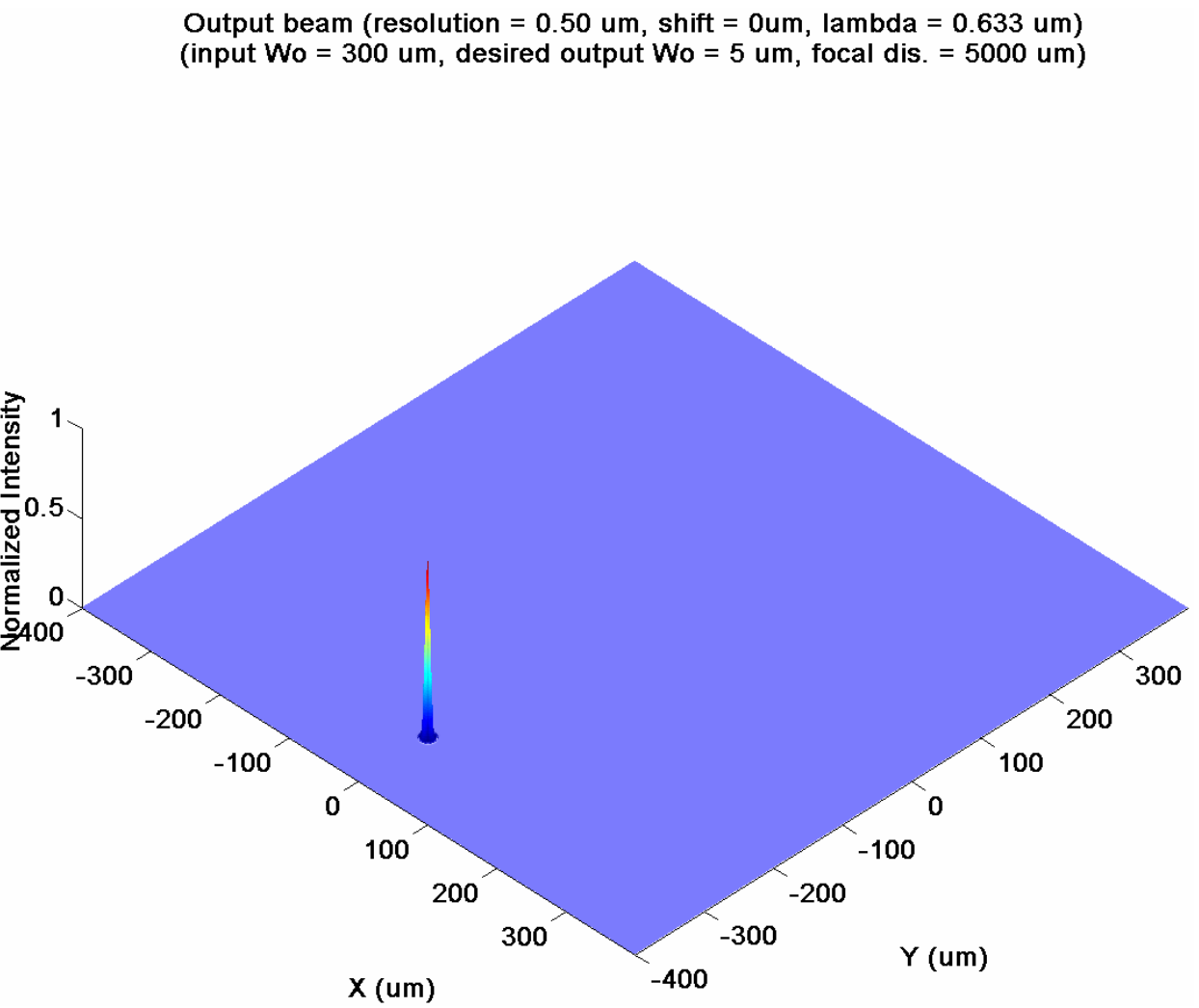

Figure 3.10: Normalized intensity plot of desired off-axis $10 \mu \mathrm{m}$ diameter Gaussian probe beam shifted $300 \mu \mathrm{m}$ from center of focal plane. 

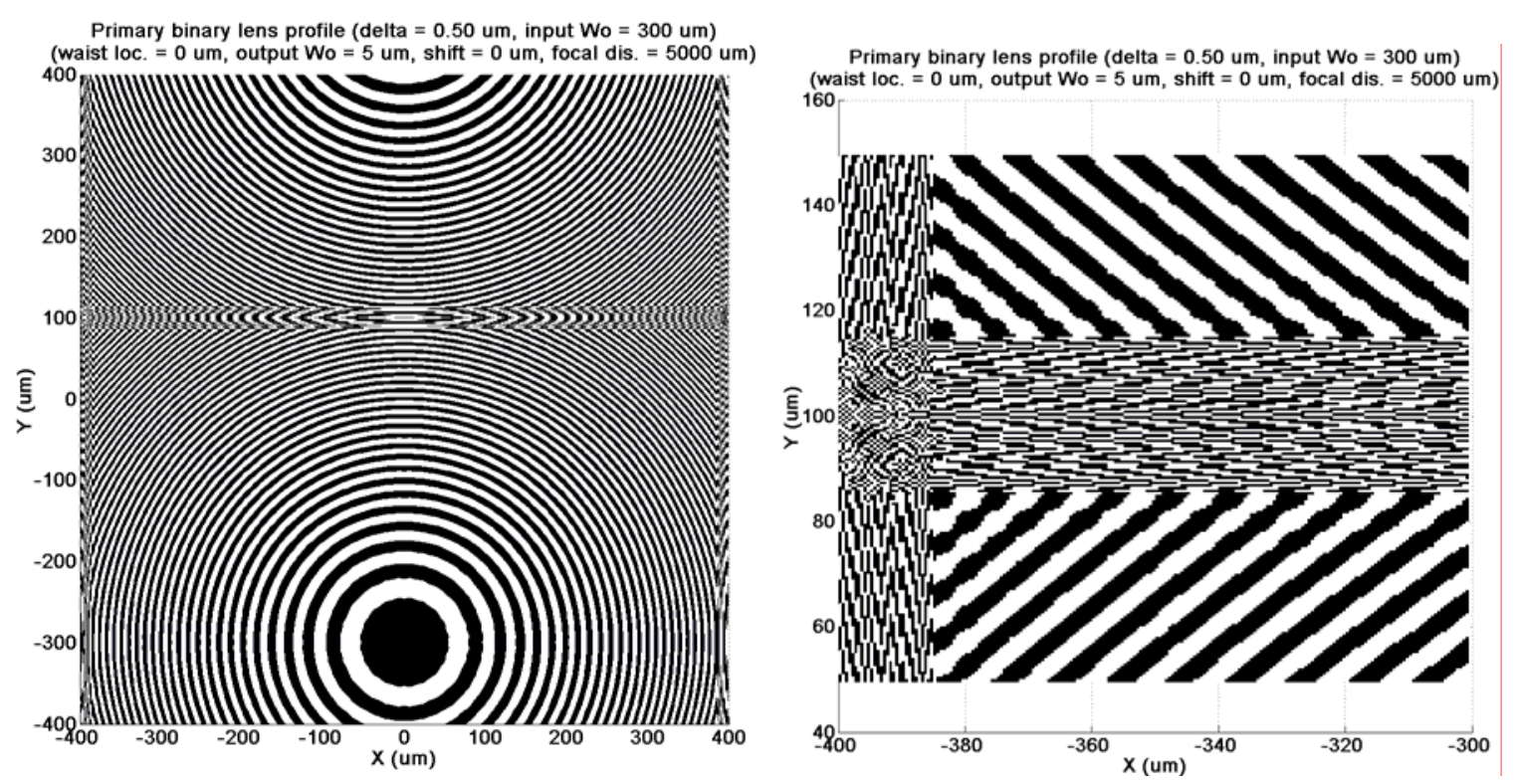

Figure 3.11: Generated single spot on-axis binary DOE.

The replication of the off axis lens pattern is believed to be a compensation to reduce the amount of comatic aberration in the output beam by diverting a portion of the beam away from the focal point. The pixilated regions are believed to be from the limitation of the $0.5 \mu \mathrm{m}$ grid used during calculation.

\subsubsection{Multiple Spot Generation}

Generation of DOEs with multiple focal points required modification of the output and was accomplished through matrix manipulation of the output array to transform a single focal point into two or more. Again, the choice of input beam and focal lengths remains the same as stated above. Figure 3.12 contains the normalized intensities of a dual on-axis output and a dual off-axis output with Figure 3.13 containing the resulting lens profiles. 

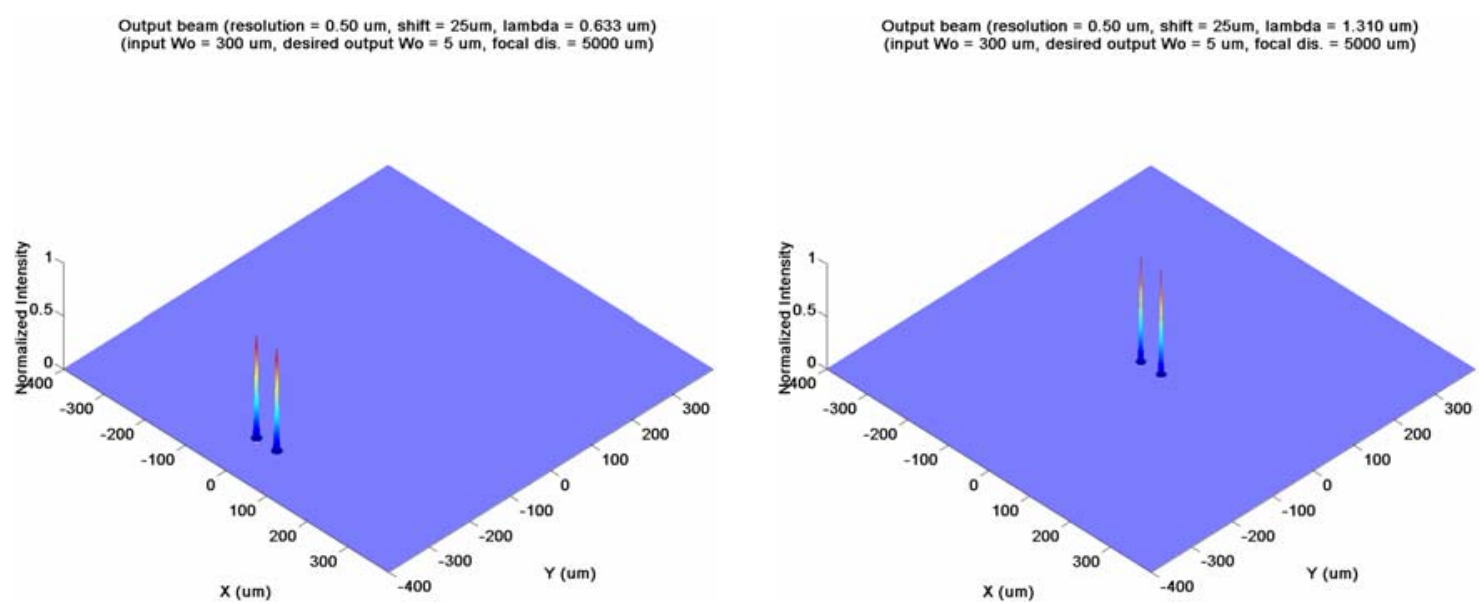

Figure 3.12: Normalized intensities of desired multiple Gaussian outputs.
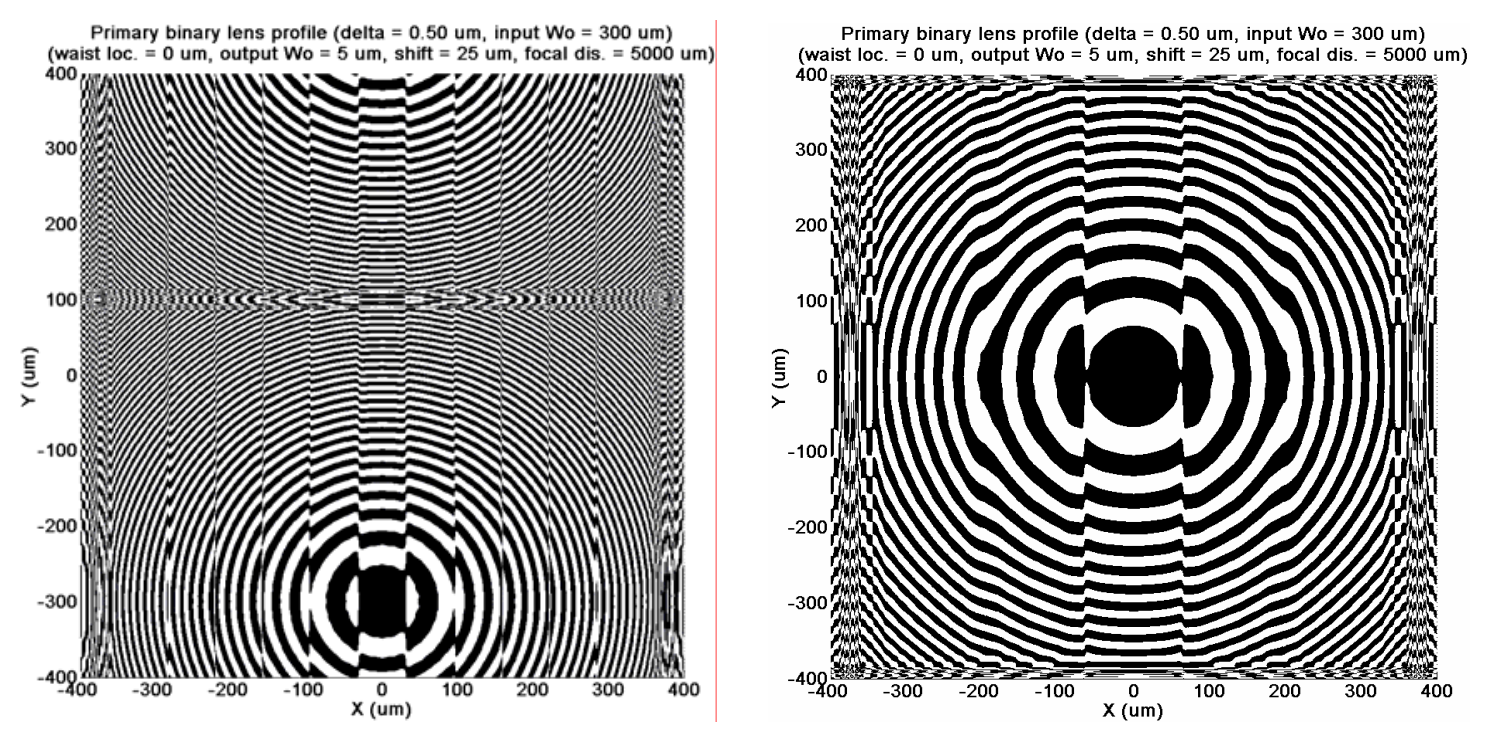

Figure 3.13: Binary dual-spot DOEs.

\subsection{Conversion from Simulation to Mask File}

ConvertToCIF.m was written in MatLab to convert individual lens arrays to the Caltech Intermediate Format (V 2.0), commonly known as a CIF file, a file type that is regularly used for importation to CAD software such as L-Edit. The CIF format was developed to act as an intermediary to translate geometries created by hand or code to a text format that can be understood by a variety of different programs [30]. The approach taken to map the lenses used $0.5 \mu \mathrm{m}$ boxes of varying lengths to preserve the $0.5 \mu \mathrm{m}$ step size employed to generate the binary lenses. ConvertToCIF.m used an edge detection 
algorithm to determine the beginning and end location of each box and convert this to a coordinate compatible with the CIF format. 


\section{Chapter 4: Fabrication and Characterization of DOE lens designs}

Chapter 4 describes the methodology of photomask layout, choice of substrate and refinement of the photolithography and reactive ion etch processes to fabricate the DOEs. Optical characterization of the DOE designs was performed once successful photolithography and RIE processes were achieved. The experimental results obtained from this optical characterization are then compared with simulation results.

\subsection{Photomask Layout and Construction}

L-Edit v10.20, written by Tanner EDA, was the CAD tool used for importation and layout of the individual lens files for the master mask used for the photolithography process to fabricate the lenses for testing. The lenses were laid out in a grid pattern with a 3mm spacing between each lens and a 3mm spacing between the outer lenses and the row/column labels to minimize extraneous diffraction effects during testing. Lenses were generated for a visible wavelength, 633nm, an infrared wavelength, $1310 \mathrm{~nm}$, and focal lengths of $1.5 \mathrm{~mm}$ and $5 \mathrm{~mm}$. The visible wavelength and the $5 \mathrm{~mm}$ focal length was chosen for ease of testing of the lenses while the infrared wavelength was chosen due to the transmittance of silicon at that wavelength. The $1.5 \mathrm{~mm}$ focal length is dependent on 
the dimensions of the proposed monitoring system detailed in section 3.4. A schematic of the final L-Edit mask layout is given in Figure 4.1 with the location and type of the lenses used for the mask given in Table 4.1. Examples of DOEs with noise regions removed and converted to L-Edit format are shown Figure 4.2.

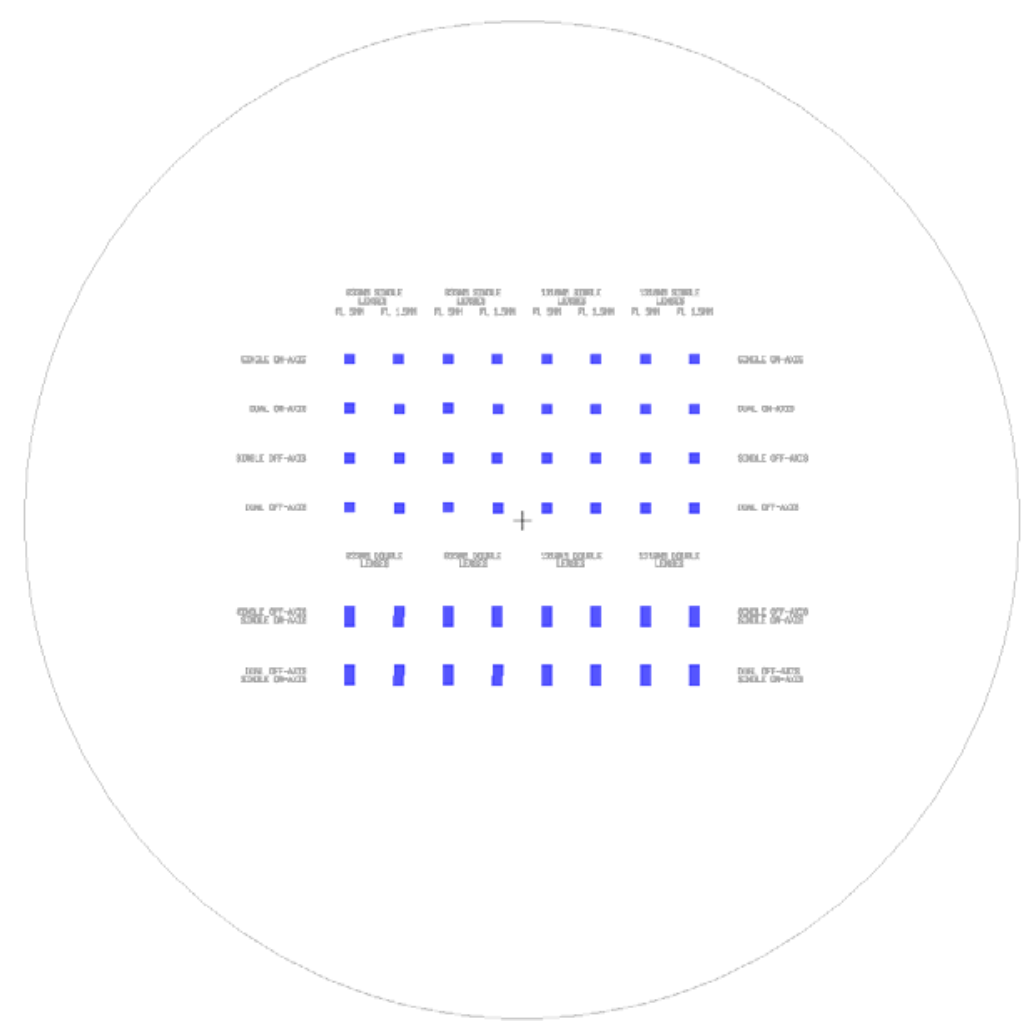

Figure 4.1: L-Edit layout of DOE optical photolithography test mask.

\begin{tabular}{|l|c|c|c|c|}
\hline & $\begin{array}{c}\mathbf{6 3 3 n m} \\
\mathbf{5 m m ~ F L}\end{array}$ & $\begin{array}{c}\mathbf{6 3 3 n m} \\
\mathbf{1 . 5 m m ~ F L ~}\end{array}$ & $\begin{array}{c}\mathbf{1 3 1 0 n m} \\
\mathbf{5 m m} \text { FL }\end{array}$ & $\begin{array}{c}\mathbf{1 3 1 0 n m} \\
\mathbf{1 . 5 m m} \text { FL }\end{array}$ \\
\hline $\begin{array}{l}\text { On-axis, } \\
\text { Single Spot }\end{array}$ & Row 1, Cols 1\&3 & Row 1, Cols 2\&4 & Row 1, Cols 5\&7 & Row 1, Cols 6\&8 \\
\hline $\begin{array}{l}\text { On-axis, } \\
\text { Dual Spot }\end{array}$ & Row 2, Cols 1\&3 & Row 2, Cols 2\&4 & Row 2, Cols 5\&7 & Row 2, Cols 6\&8 \\
\hline $\begin{array}{l}\text { Off-axis, } \\
\text { Single Spot }\end{array}$ & Row 3, Cols 1\&3 & Row 3, Cols 2\&4 & Row 3, Cols 5\&7 & Row 3, Cols 6\&8 \\
\hline $\begin{array}{l}\text { Off-axis, } \\
\text { Dual Spot }\end{array}$ & Row 4, Cols 1\&3 & Row 4, Cols 2\&4 & Row 4, Cols 5\&7 & Row 4, Cols 6\&8 \\
\hline $\begin{array}{l}\text { Dual lens, } \\
\text { Single Spot }\end{array}$ & Row 5, Cols 1\&3 & Row 5, Cols 2\&4 & Row 5, Cols 5\&7 & Row 5, Cols 6\&8 \\
\hline $\begin{array}{l}\text { Dual lens, } \\
\text { Dual Spot }\end{array}$ & Row 6, Cols 1\&3 & Row 6, Cols 2\&4 & Row 6, Cols 5\&7 & Row 6, Cols 6\&8 \\
\hline
\end{tabular}

Table 4.1: Location of DOEs by type. 
a)

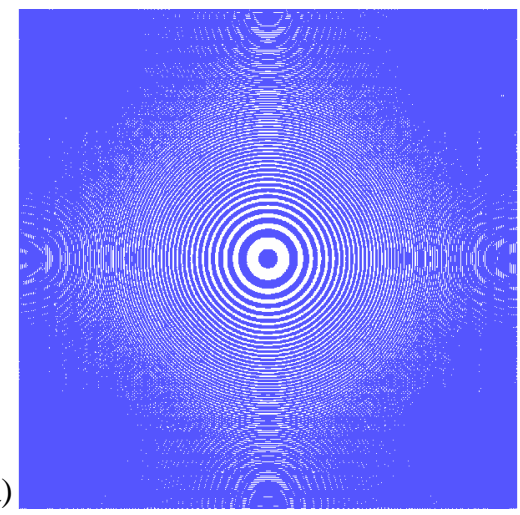

c)

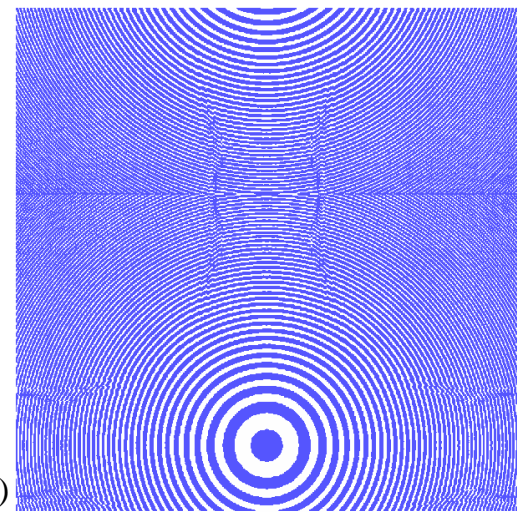

e)

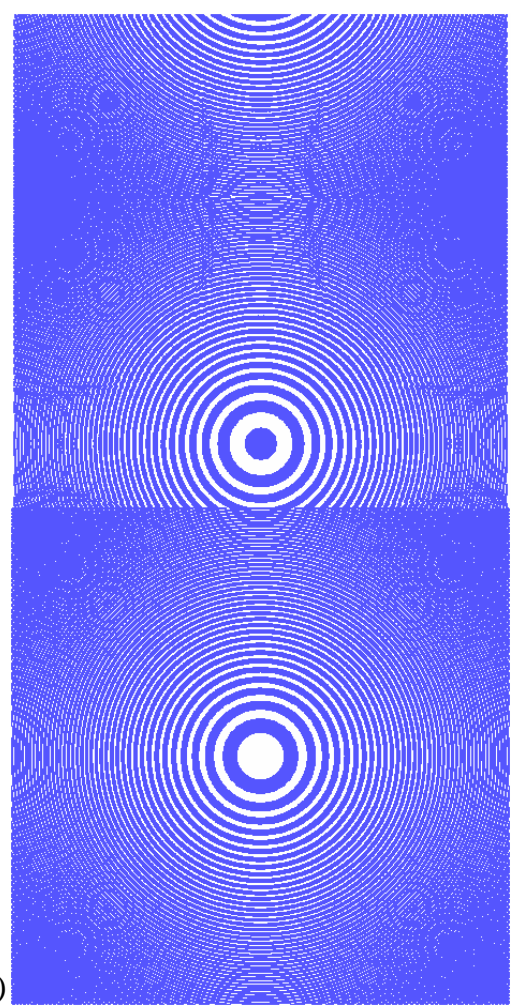

b)
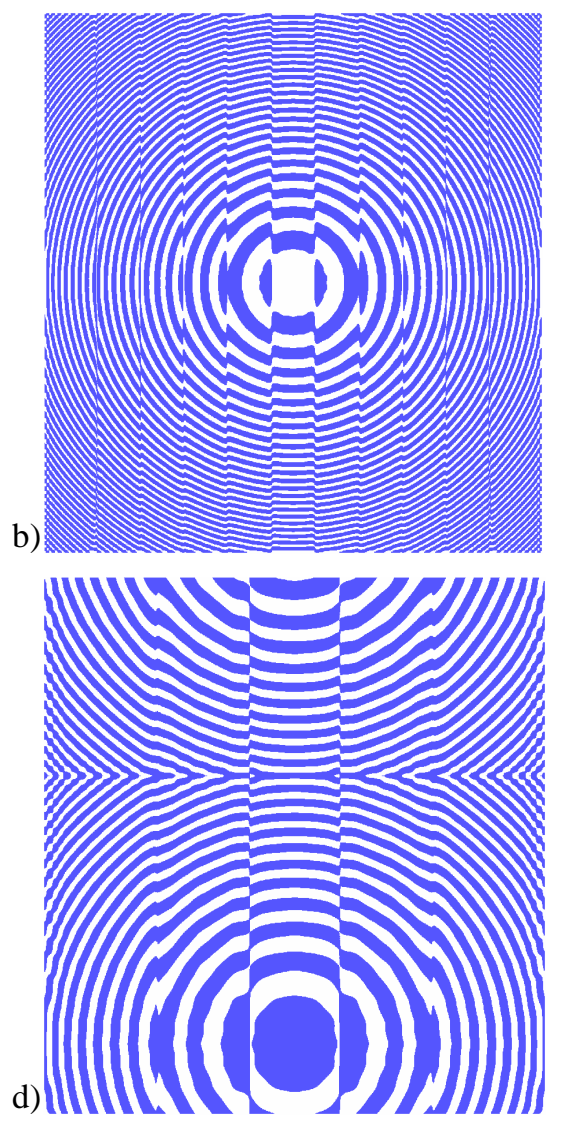

Figure 4.2: Examples of on-axis and off-axis DOEs converted to L-Edit.

a) Single probe, on-axis, $633 \mathrm{~nm}, 1.5 \mathrm{~mm}$ focal length.

b) Dual probe, on-axis, 633 nm, $5 \mathrm{~mm}$ focal length.

c) Single probe, off-axis, $633 \mathrm{~nm}, 1.5 \mathrm{~mm}$ focal length.

d) Dual probe, off-axis, $1310 \mathrm{~nm}, 5 \mathrm{~mm}$ focal length.

e) Input and output DOEs for $633 \mathrm{~nm}, 1.5$ focal length. 
The resulting L-edit layout was converted to a GDSII format and sent to the Penn State Nanofabrication Facility (Nanofab), a member of the National Nanofabrication Users Network (NNUN), for fabrication of a 5-inch chrome/quartz photomask using an ebeam write system. The quartz substrate is used because it absorbs less energy at the ultraviolet wavelength of 320nm which was used for photoresist exposure than the alternative soda lime mask substrate. The transparency of the quartz at the exposure wavelength greatly decreases exposure time minimizing pattern distortion created by vibration during exposure. The Nanofab facility uses blanks pre-coated with photoresist and the 5 -inch photomask had to be used to achieve the $0.5 \mu \mathrm{m}$ features of the lens design even though the lens area only covered a portion of the mask area.

\subsection{Optical Substrate and Description of Lens Fabrication}

The choice of an optical substrate and etching method must be made once the generation and simulation of the lens mask has been completed. Three inch diameter Schott Borofloat wafers were chosen as the substrate due to its cost, availability and use in other projects where DOEs may be of interest, such as microfluidic elements. Schott Borofloat is a borosilicate flat glass made in a float process and is similar in chemical composition to Corning Pyrex 7740 giving it high resistance to attack from water, acids and alkalis. The chemical composition is shown in Figure 4.3 [31].

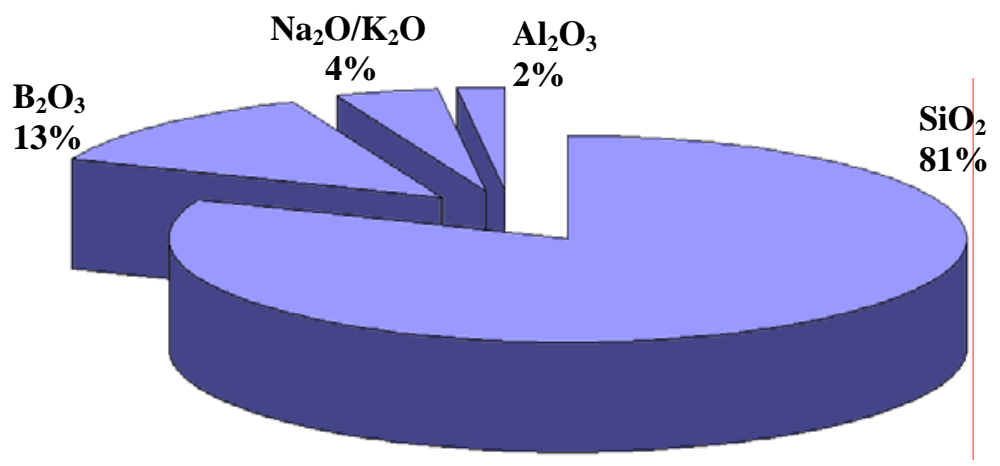

Figure 4.3: Chemical composition of Borofloat [31].

The optical characteristics of Borofloat are ideal for use as a lensing material for ranges from ultraviolet to infrared as shown in Figures 4.4, 4.5, and 4.6 [32,33,34]. 


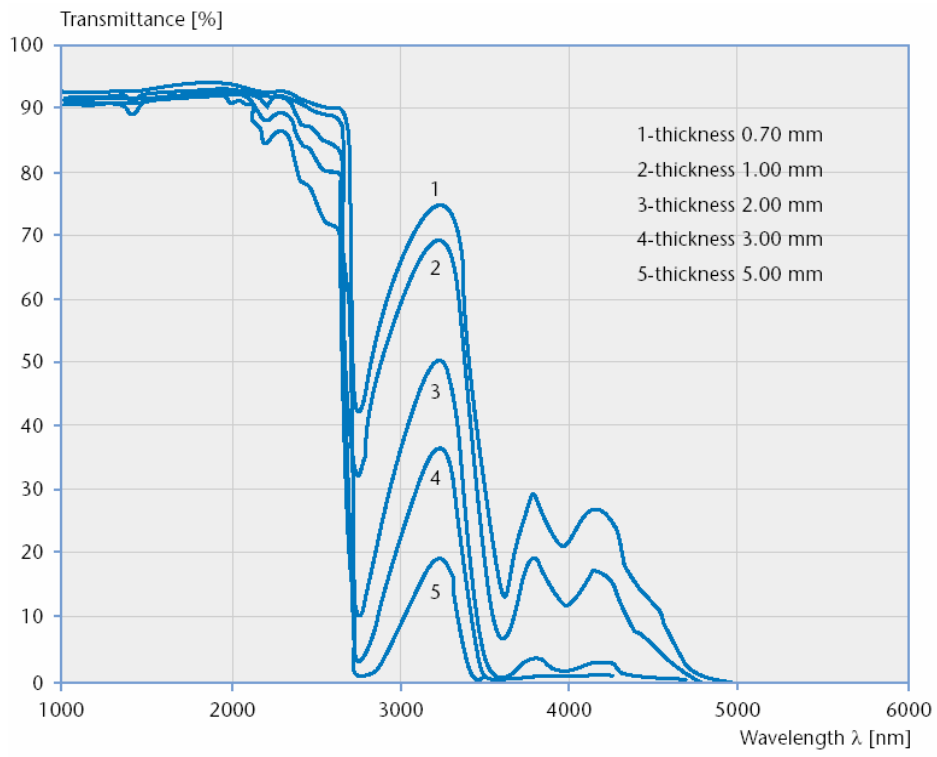

Figure 4.4: Borofloat transmittance vs. IR wavelength [33].

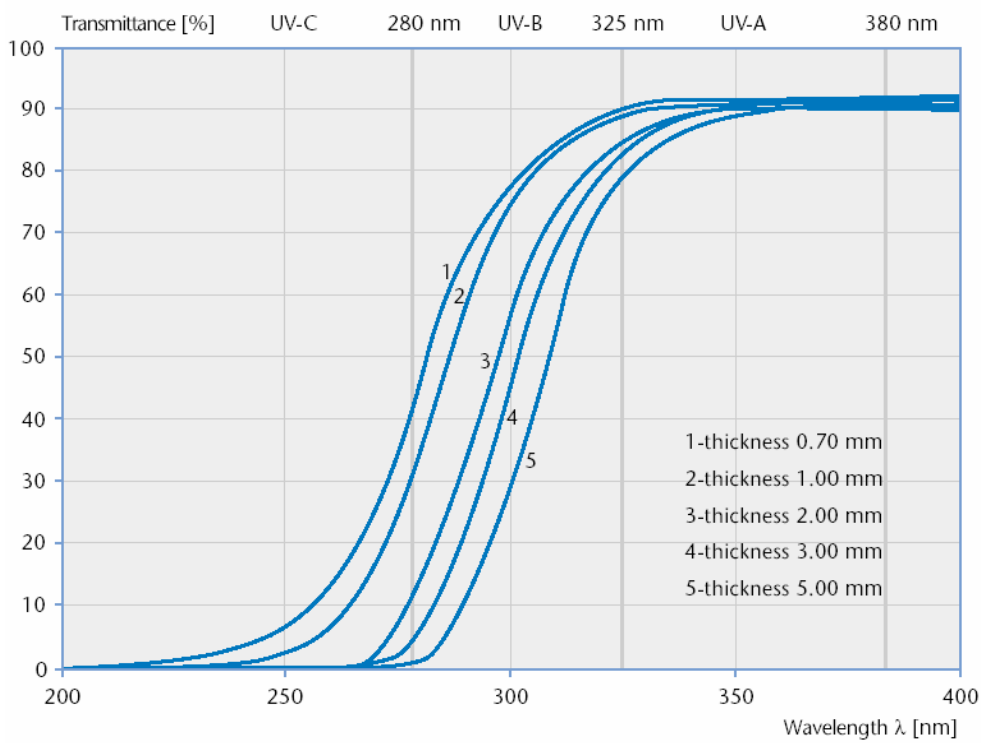

Figure 4.5: Borofloat transmittance vs. UV wavelength [32]. 


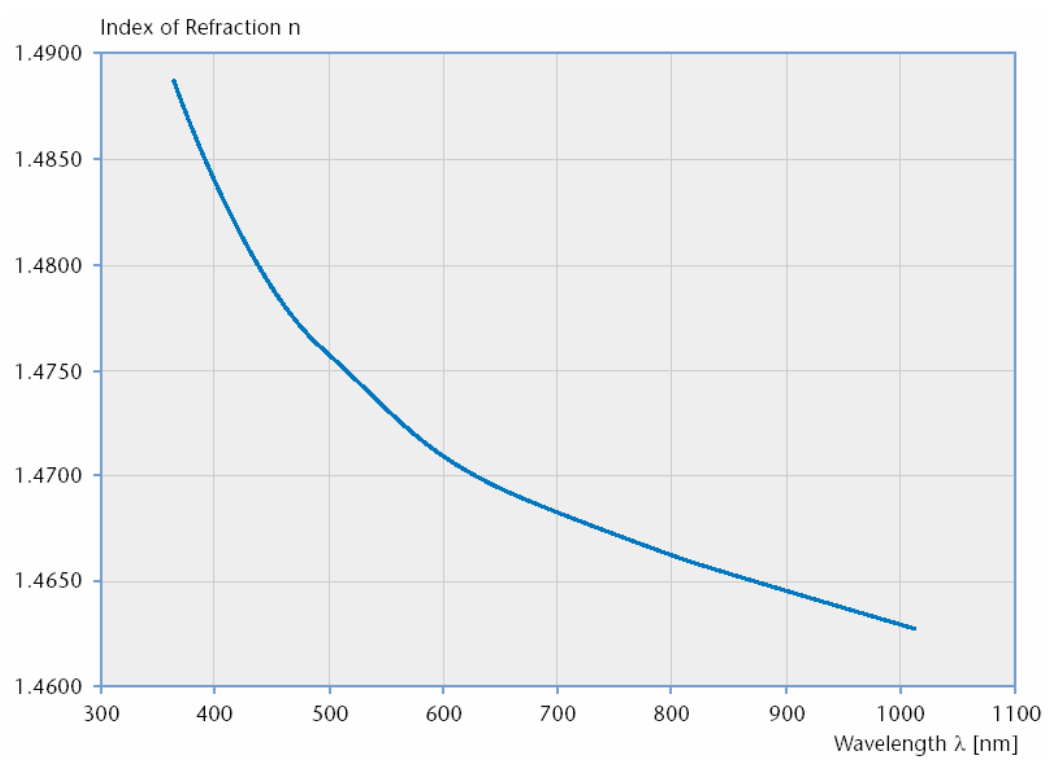

Figure 4.6: Borofloat index of refraction vs. wavelength [34].

\subsubsection{Photolithography}

The method selected for lens fabrication was based on optical photolithography process to transfer the pattern to the photoresist which is then subsequently transferred to the substrate with a plasma etch process to fabricate the lenses. The choice of photolithographic process is determined by the smallest feature size to be imaged and type of photoresist used, which can be classified as either a positive, negative or image reversal. Photoresist (PR) is composed of a resin or base material, a photoactive component (PAC) which determines the response of the PR to ultraviolet (UV) exposure and solvent which determines the viscosity and other physical characteristics of the resist[35]. The PAC of a positive resist acts as a dissolution inhibitor until exposed to UV radiation of sufficient energy to break the polymer bonds of the resist. The exposed areas will then dissolve in a developer solution more easily than those regions not exposed, resulting in the desired pattern. A negative resist works in the opposite manner, with the unexposed regions dissolving in the developer solution. Figure4.7 illustrates a positive and negative photoresist process. 

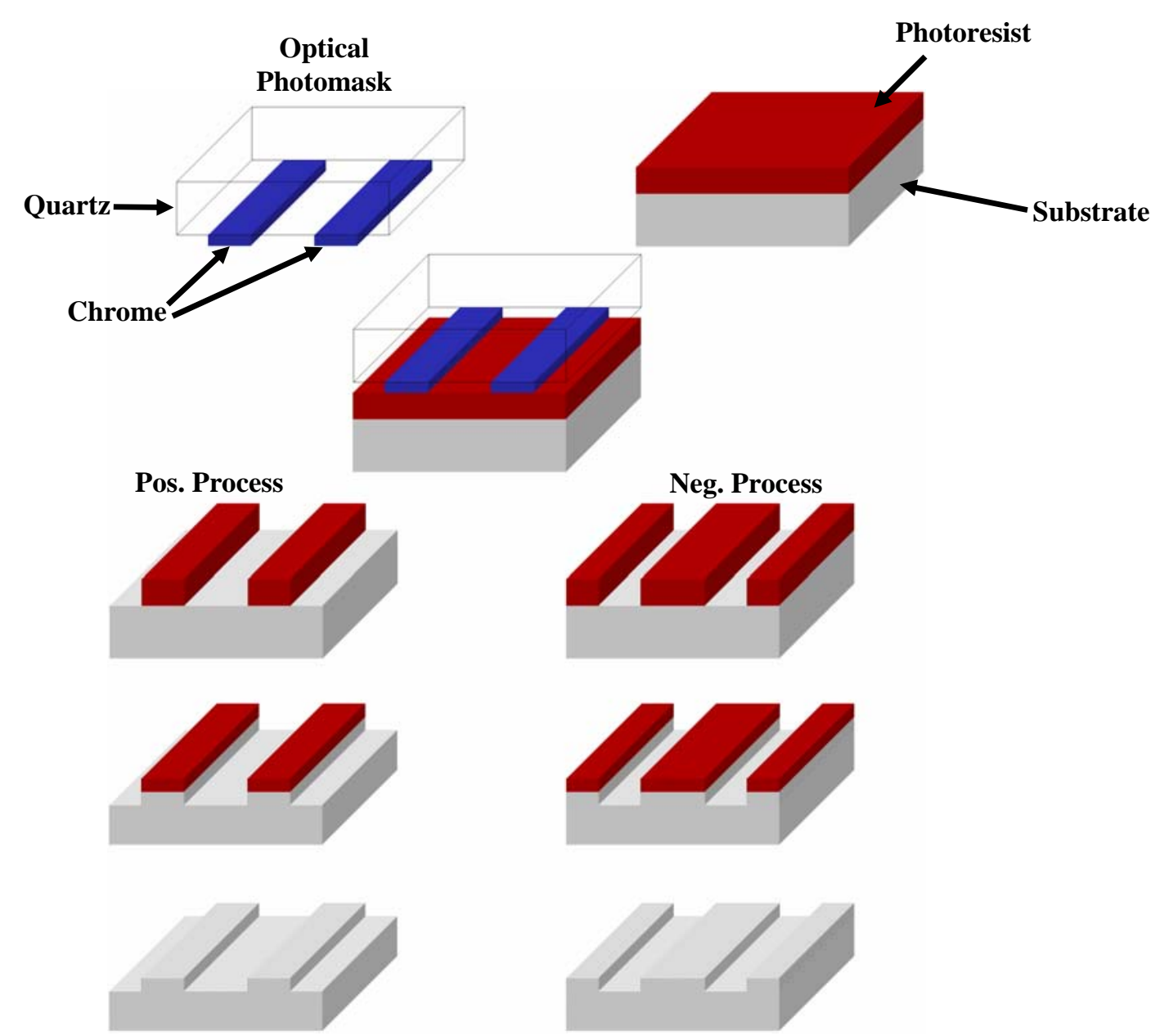

Figure 4.7: Positive and negative photolithography.

\subsubsection{Reactive Ion Etch}

The two basic methods to etch semiconductor materials used for MEMS devices, ICs and DOEs are wet etch and dry etch. A wet etch is usually performed by submerging the target into a solution that will chemically react with the material that is to be removed. Wet etching has many undesirable characteristics for DOEs including its isotropic nature which increases the difficulty of achieving vertical sidewalls, contamination from particulate in the etchant solution leading to defects, lack of resolution of features smaller than $2 \mu \mathrm{m}$ and excessive use of chemicals [35]. A dry etch process uses a plasma to physically and/or chemically remove unwanted material and can be broken down into the subcategories of high-pressure plasma etch, ion milling and reactive ion etch. The rate, selectivity and side-wall characteristics of the etch depend on the process gases, pressure 
and power used to generate the plasma. Some advantages of plasma etching include less susceptibility to temperature changes, more control over the start and stop of the etch, less contamination, less chemical waste and a more anisotropic profile [35]. A RIE process is a combination chemical/physical etch process and was chosen due to the necessity of vertical side-walls, the $0.5 \mu \mathrm{m}$ features found in the lenses and the availability of the Oxford PlasmaLab $80+$ reactive ion etch system in our shared microfabrication lab.

\subsubsection{Lens Fabrication}

Preliminary photolithography and RIE tests were performed using an available optical mask during the period of time when the DOE optical mask was being fabricated in order to get a head start on process characterization. The initial photolithography process was based on Clariant guidelines for using AZ5214E photoresist followed by a RIE. A generic flow diagram of the lens fabrication process using image reversal is shown in Figure 4.8.

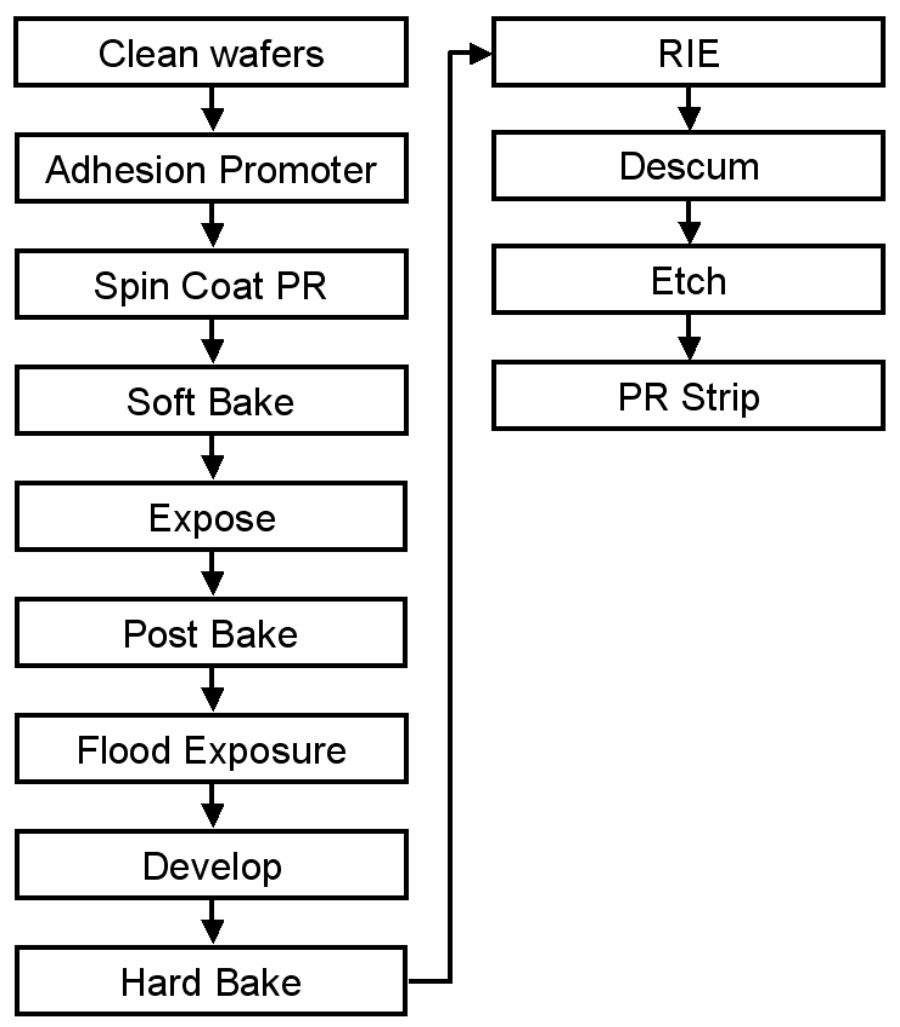

Figure 4.8: Generic image reversal photolithography and RIE etch process. 


\section{Photolithography}

The lens fabrication process begins by cleaning the wafer sample with organic degrease and heavy metal ion removal procedures. The degrease entails submersion in acetone, methanol and de-ionized (DI) water for 5 minutes respectively followed by the heavy metal ion removal which consists of a 30 minute bath in SummaClean and a 5 minute flow of DI water. This is followed by a 30 minute dehydration oven bake at $100^{\circ} \mathrm{C}$ to remove adsorbed water from the surface layers of the wafer [35].

The next step is the application of thin layer of hexamethyldisilazane (HMDS) via the Yield Engineering Systems LP-III vacuum vapor deposition oven. The standard program for the oven was modified by first increasing the initial vacuum pump time from 2 minutes to 5 minutes to reach the proper operating pressure and second, by increasing the HMDS deposition time from 5 minutes to 6 minutes. HMDS is a photoresist adhesion promoter that reduces the chance of photoresist lift-off during development. This lift-off can be attributed to the large spacing between un-bonded molecules of the photoresist polymer [11]. While de-adhesion is not a significant problem with patterns consisting of primarily large features, the application of HMDS is crucial here because of the relatively small features of the lenses. Figure 4.9 is an example of post development de-adhesion attributed to insufficient or lack of HMDS and incomplete UV exposure. 


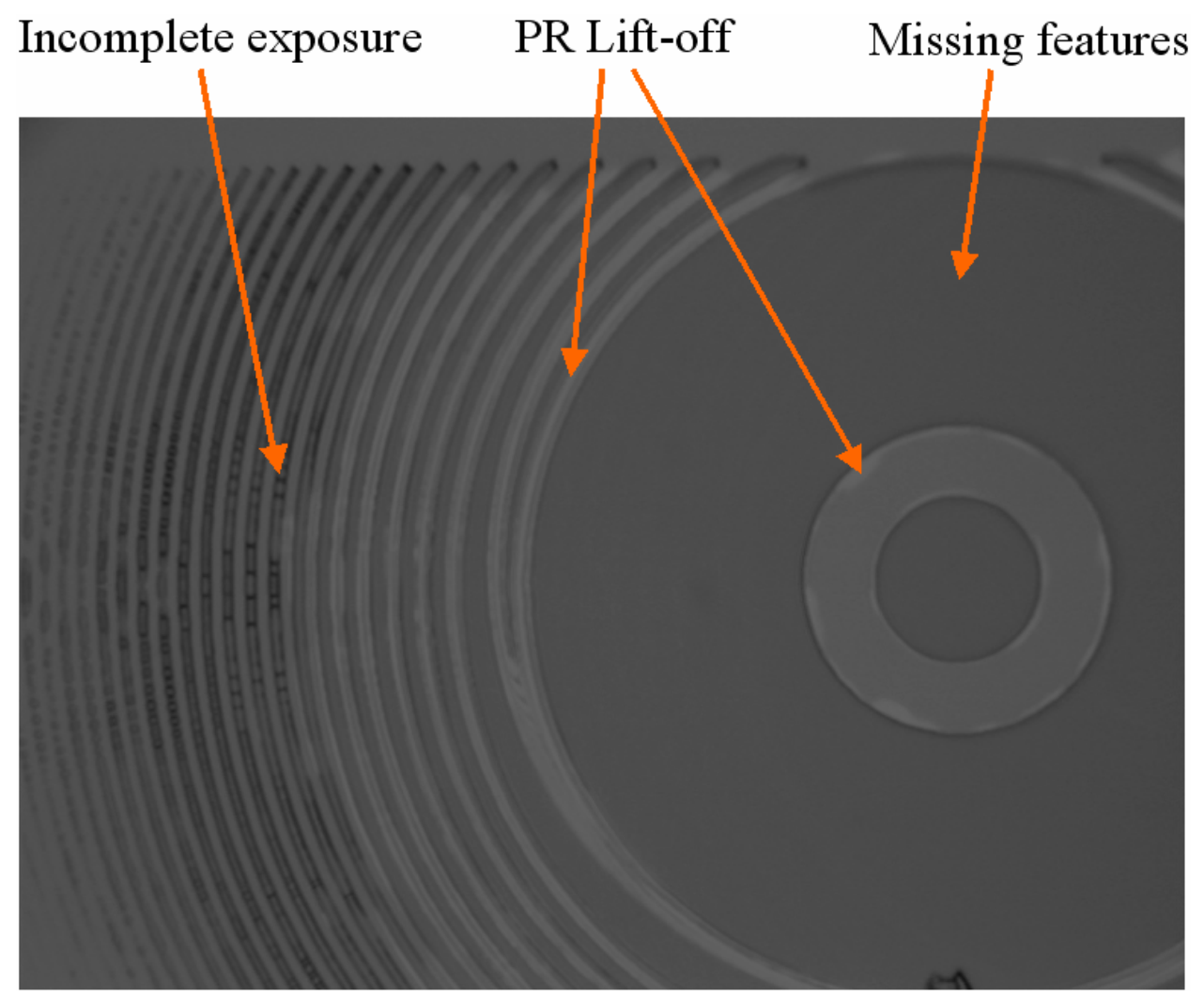

Figure 4.9: PR de-adhesion, lift-off and incomplete exposure.

AZ5214E photoresist can be used in positive as well as image reversal photolithography depending on the needed application and is capable of attaining submicron features [36]. A conscious choice was made to have the optical photomask fabricated as a light field to aid in alignment issues. The image reversal process was used to protect the Borofloat substrate during etching, leaving only the areas of the lenses exposed to the RIE plasma. Application of AZ5214E was performed using a spin coating method at $5000 \mathrm{rpm}$ resulting in thicknesses of $\sim 1.5 \mu \mathrm{m}$ to $\sim 1.8 \mu \mathrm{m}$. This differs from the expected thickness of $1.25 \mu \mathrm{m}$ but may be explained three ways:

- photoresist age - AZ5214E used is an older sample and may have an increasing viscosity with depth of bottle.

- temperature - AZ5214E is stored in a refrigerated environment and may not have been allowed to warm properly before application.

- improper spin speed - The spinner used for coating the sample may need to be recalibrated to ensure correct speed. 
Spin coating the wafer causes a bead of thicker PR to form on the outer edge of the substrate and must be removed prior to the soft bake to ensure intimate contact of the mask during hard contact exposure. This is accomplished using an acetone soaked cotton swab that is carefully rubbed along the outer edge of the sample. Densification of the photoresist is then performed via a soft bake to remove solvents, increasing the optical sensitivity, by placing the wafer on a hot plate set at $100^{\circ} \mathrm{C}$ for 60 seconds.

Pattern transfer is accomplished by a UV exposure at 320nm using the Karl SUSS MA6 mask aligner with calculation of exposure time being based on measured power of the MA6 and guidelines for the photoresist used. The MA6 has the ability to perform a proximity exposure, leaving a space between the mask and wafer, soft contact, hard contact and vacuum contact, which uses a vacuum seal to place the mask in intimate contact with the wafer. Contact lithography is acceptable for large features (100s of microns) with large gaps between but vacuum contact is needed when the pattern features are on the micron to sub-micron scale to minimize diffraction effects. An example of diffraction modifying a pattern during soft contact exposure is shown in Figure 4.10.

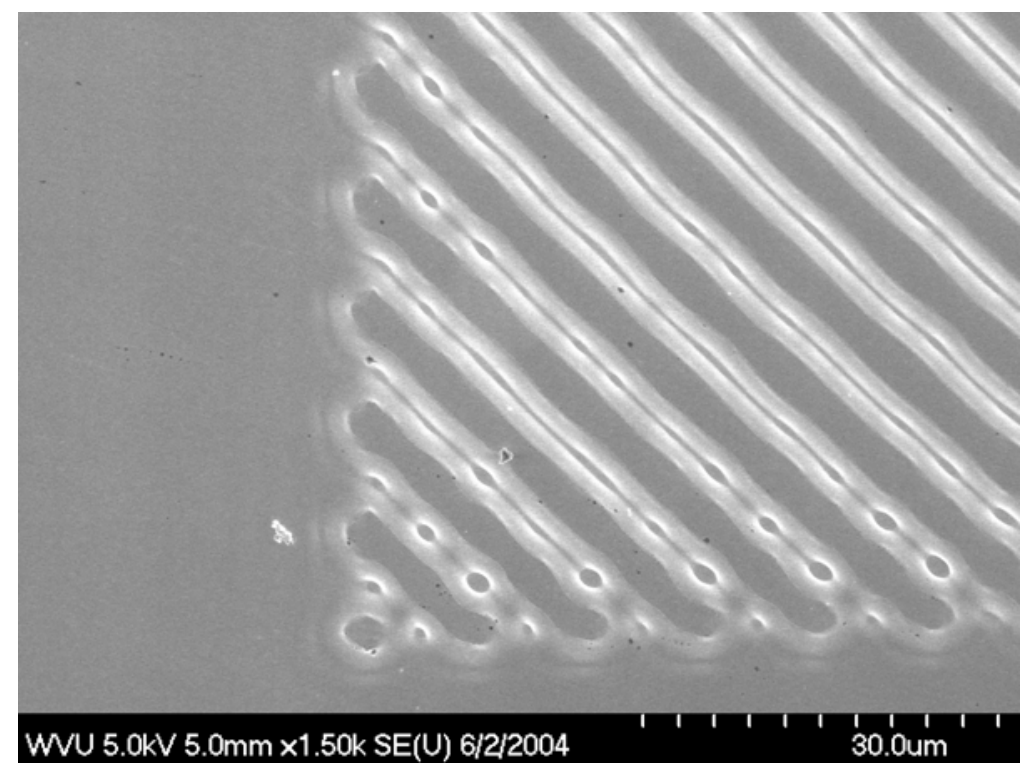

Figure 4.10: Diffraction effects in small features during soft contact exposure.

The image reversal is completed with a post exposure bake by placing the exposed wafer on a different hot plate set at $120^{\circ} \mathrm{C}$ for 2 minutes followed by a flood exposure at 
365nm. The post exposure bake is the most important step because it activates a crosslinking mechanism within the PR to enable the image reversal [37]. The temperature and time for the hard bake will differ depending on wafer thickness and composition. The Clariant AZ5214E datasheet is included in Appendix B for reference.

The developer used to complete the photolithography process was AZ400K, a potassium based solution, diluted to a 1:6 ratio of developer to DI water to increase the contrast and sensitivity of the process. Agitation should be kept to a minimum during development to reduce the chance of small features detaching from the substrate. The sample is then placed in a container of DI water to stop further development of the pattern through dilution. The nitrogen stream used to dry the sample after development should not be directed such that high pressure damages the resulting pattern and can be accomplished by holding the nozzle farther from the substrate and aiming the stream at a slight angle. A combination of either incorrect initial exposure, second bake time/temperature or development times can lead to sloped sidewalls as seen in Figure 4.11.

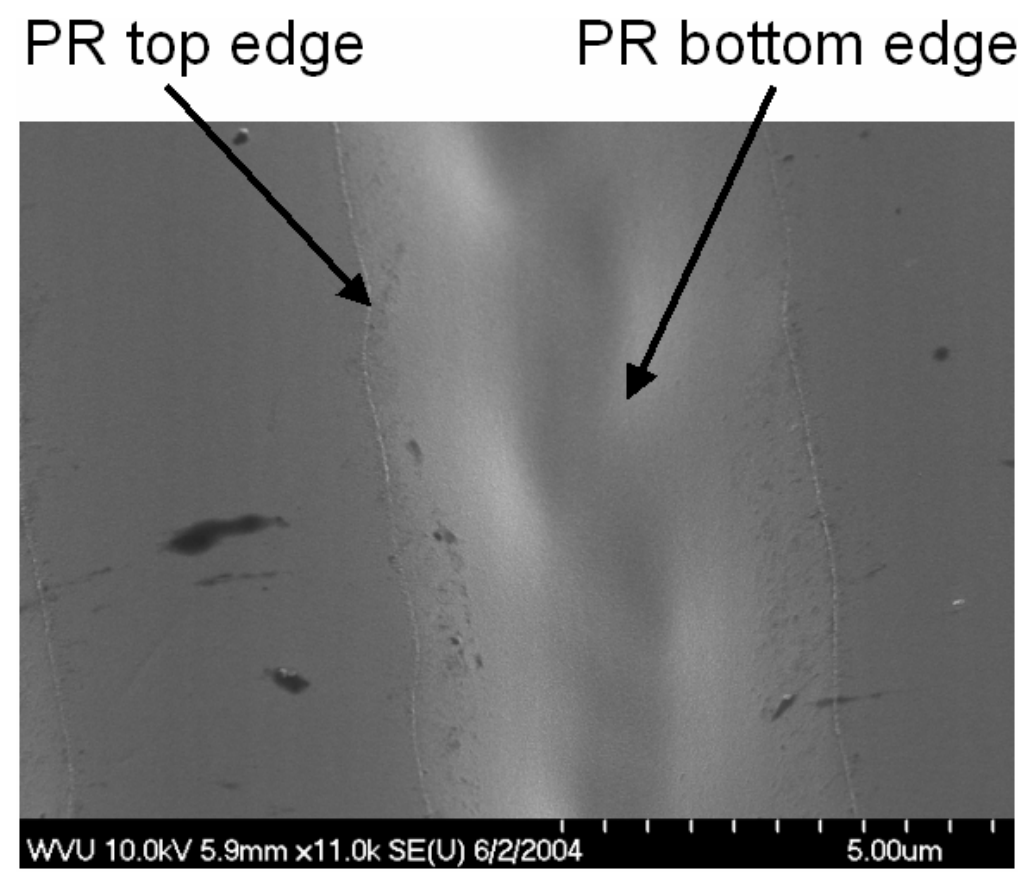

Figure 4.11: Sloped PR sidewalls. 
It should be noted that values for bake times, UV exposure time and development time are empirically derived through trial and error based on guidelines given by the photoresist manufacturer. Feature sizes on the scale of $0.5 \mu \mathrm{m}$ were able to be resolved with good PR adhesion and sidewall development using the photolithography process given in Table 4.2. A result from this process is shown in Figure 4.12. It can be noted that the transitions in the mask are clearly visible, indicating submicron resolution. Also, sidewalls are not evident in the optical micrograph.

\section{Image Reversal Photolithography using AZ5214E}
1. 30 minute Summa Clean
2. 5 minute acetone soak.
3. 5 minute methanol soak.
4. 5 minute DI soak.
5. Dry with pressurized $\mathrm{N}_{2}$.
6. 30 minute dehydration bake @100 $\mathrm{C}$.
7. 6 min HMDS deposition
(full deposition time is approx 35-40 min).
8. Spin AZ5214E @5000 rpm (room temperature).
9. Soft bake on hot plate @ $100^{\circ} \mathrm{C}$ for 60 seconds.
10 Expose for $37.5 \mathrm{~mJ} / \mathrm{cm}^{2}$, 20.8 seconds at $1.8 \mathrm{~mW} / \mathrm{cm}^{2}$ on MA-6 (320nm).
11 Post bake on hot plate @120 ${ }^{\circ} \mathrm{C}$ for 2 min.
12 Flood expose $2 \mathrm{~J} / \mathrm{cm}^{2}$ on the M1 (365nm).
13 Develop with AZ400K 1:6 100 seconds.
1430 min dehydration bake @ $100^{\circ} \mathrm{C}$.

Table 4.2: Final photolithography process which yielded $0.5 \mu \mathrm{m}$ features. 


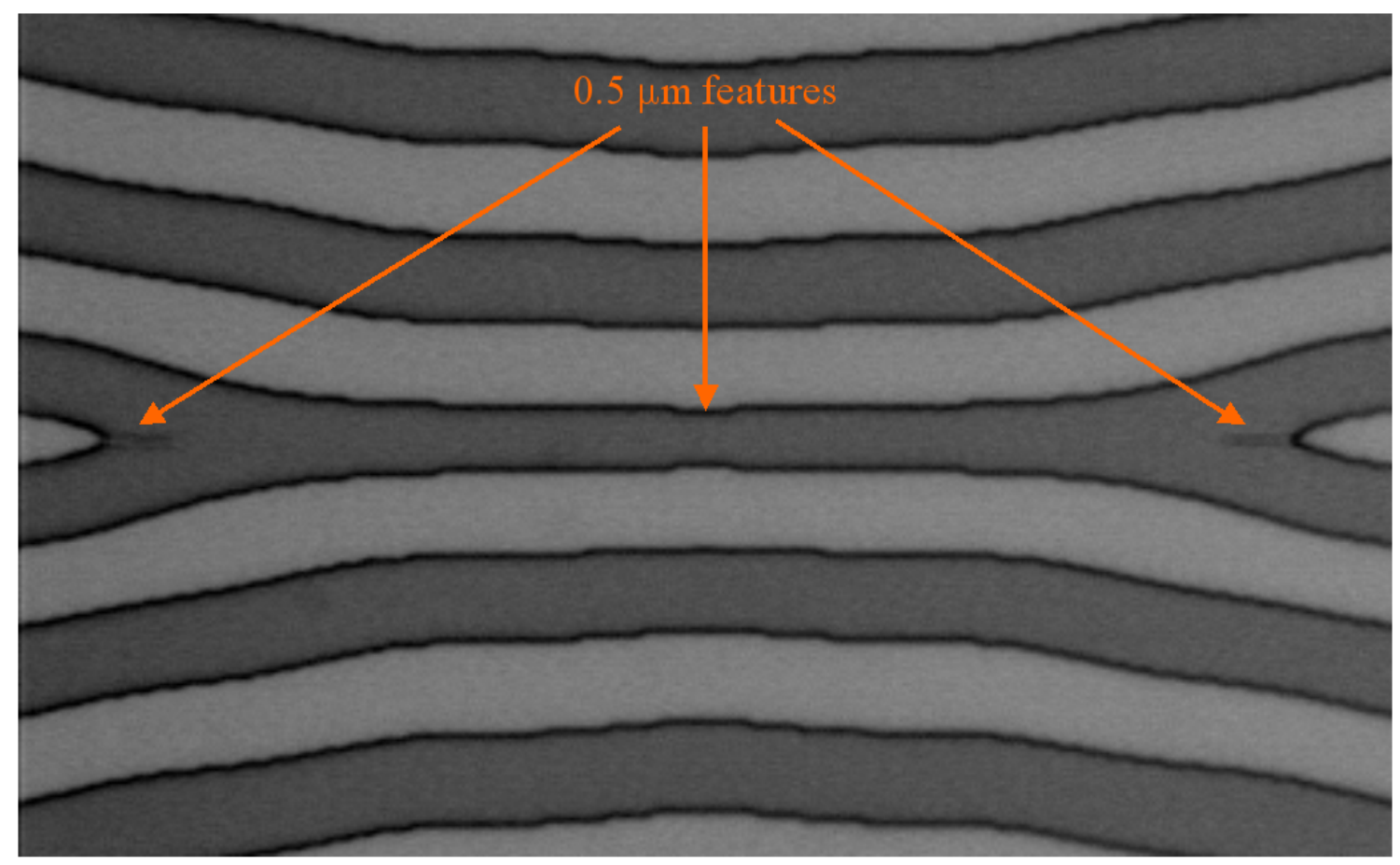

Figure 4.12: PR pattern containing $0.5 \mu \mathrm{m}$ features.

\section{Reactive Ion Etch}

The RIE process begins with an oxygen plasma “descum” to remove residual HMDS and PR from the open developed areas of the PR pattern prior to substrate etching. The descum is performed with the following recipe [11]:

\begin{tabular}{|c|c|c|c|c|c|}
\hline Flow Rate & Species & Pressure & RF Power & ICP Power & Etch Time \\
\hline $30 \mathrm{sccm}$ & $\mathrm{O}_{2}$ & $300 \mathrm{mT}$ & $150 \mathrm{~W}$ & $0 \mathrm{~W}$ & 30 secs \\
\hline
\end{tabular}

where the flow rate of sccm is standard cubic centimeters per minute, chamber pressure, $\mathrm{mT}$, is measured in milliTorr, the power between the cathode and anode of the system is measured in watts of forward RF power and the duration of the etch is measured in minutes and seconds. This oxygen plasma also etches the photoresist but removal is minimal due to short exposure time to the plasma.

Prior work with Fresnel lens fabrication on quartz substrates with trifluoromethane, $\mathrm{CHF}_{3}$, as the primary plasma etchant was used as the initial starting point for the investigation for using RIE of Borofloat [11]. Focus was shifted to the use of carbon 
tetraflouride, $\mathrm{CF}_{4}$, and $\mathrm{O}_{2}$ based on [38] and plasma etch tests performed on Pyrex using the following recipe

\begin{tabular}{|c|c|c|c|c|c|}
\hline Flow Rate & Species & Pressure & RF Power & ICP Power & Etch Time \\
\hline $25 \mathrm{sccm} / 5 \mathrm{sccm}$ & $\mathrm{CF}_{4} / \mathrm{O}_{2}$ & $6 \mathrm{mT}$ & $100 \mathrm{~W}$ & $0 \mathrm{~W}$ & $16: 52$ min:secs \\
\hline
\end{tabular}

which should have resulted in an etch depth of approximately 523nm. Measurements using the AlphaStep profilometer suggest an etch rate of $19 \mathrm{~nm} / \mathrm{min}$ based on an average etch depth of 314nm compared to an etch rate of 31nm/min from [38]. Disparity between experimental etch rate and that found in literature could be due to differences in the equipment used to perform the RIE process. The sample that was etched using this recipe is shown in Figure 4.13

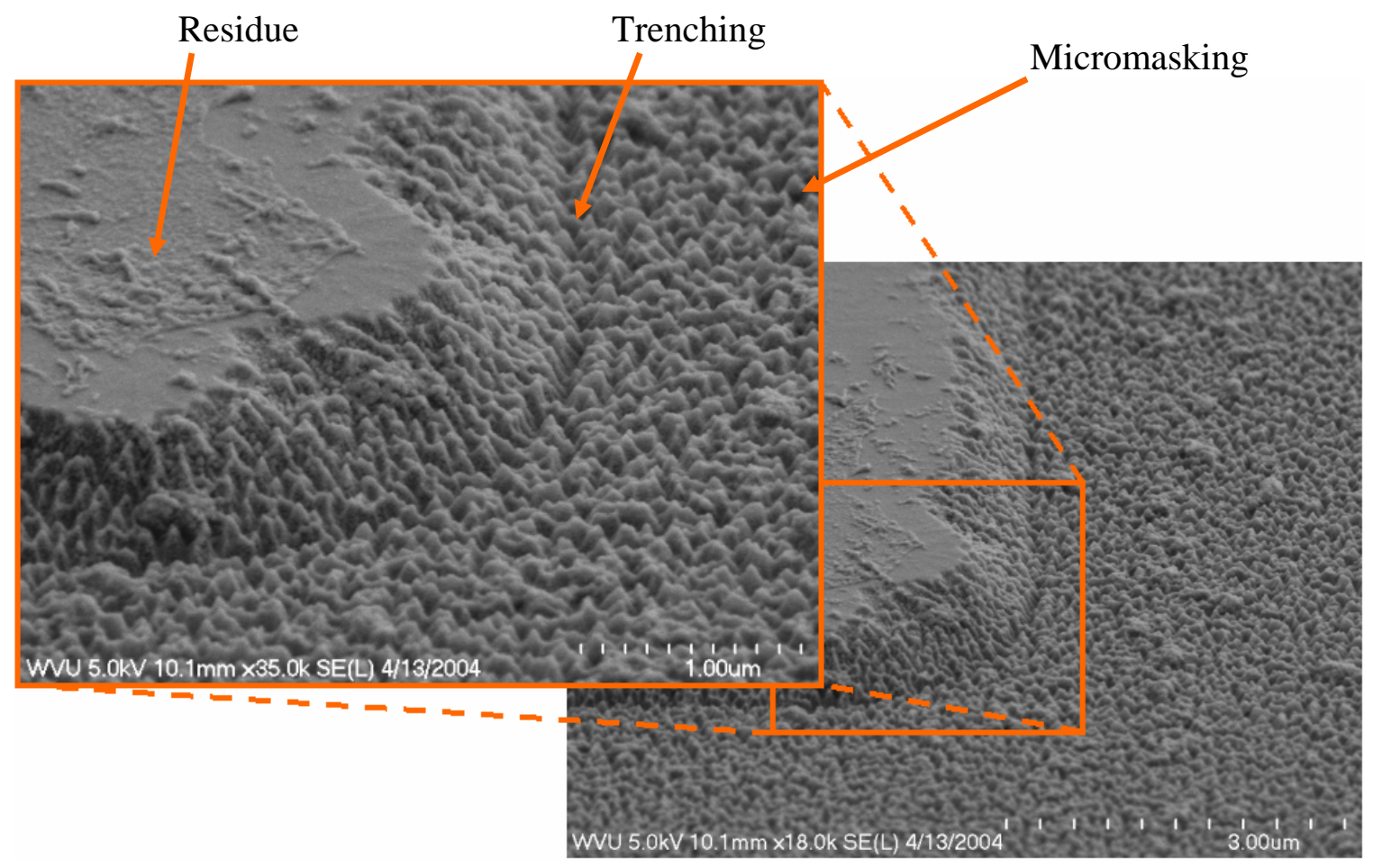

Figure 4.13: Examples of trenching, micromasking and improper PR development resulting in sloped sidewalls.

The sample in Figure 4.13 was patterned using a positive PR process with soft contact setting on the MA6. The extreme slope of the etched sidewall can be attributed to a combination of underexposure and over development of the PR prior to etch. The 
roughness of the etch is believed to be micromasking and/or redeposition of nonvolatile compounds which do not chemically react with the plasma etchant. The distinct line at the base of the slope is an example of "trenching", an effect that occurs when plasma ions strike a tapered sidewall and reflect back toward the base [35]. The residue located in the unetched region could be redeposition of nonvolatile material or residual PR that was not completely removed.

Further exploration of the literature indicated that lower chamber pressures and lower flow rates would move the etch toward a more physical ion bombardment as opposed to a chemical removal of material [35,39]. The process described used sulfur hexafluoride, $\mathrm{SF}_{6}$, as the etch species and operated at a chamber pressure of 0.2 Pascal (Pa) or approximately $1.5 \mathrm{mT}$ with a flow rate of $7 \mathrm{sccm}$. The system also incorporated an inductively coupled plasma (ICP) at $150 \mathrm{~W}$ with a strong permanent magnet in addition to the RF power of $140 \mathrm{~W}$ to achieve etch profiles with nearly vertical sidewalls and an extremely smooth etched surface. This combination of parameters permitted the generation of a densified plasma that generated ions with a long mean free path and emphasized ion bombardment over chemical processes [39].

Implementation of a similar process using $\mathrm{CF}_{4}$ was undertaken with the following recipe:

\begin{tabular}{|c|c|c|c|c|c|}
\hline Flow Rate & Species & Pressure & RF Power & ICP Power & Etch Time \\
\hline 10 sccm & $\mathrm{CF}_{4}$ & $2 \mathrm{mT}$ & $140 \mathrm{~W}$ & $150 \mathrm{~W}$ & $13: 24$ min:secs \\
\hline
\end{tabular}

This process was performed but with limited results due to physical limitations the Oxford PlasmaLab 80+, primarily due to the minimum chamber pressure of approximately $8 \mathrm{mT}$. A plasma was formed at a flow rate of $10 \mathrm{sccm}$ but the plasma flickered periodically throughout the etch process. This could be attributed to the difference between the number of fluorine ions, electrons and molecular radicals that can be produced by $\mathrm{CF}_{4}$ and $\mathrm{SF}_{6}$ to create a stable plasma at lower chamber pressures. The inclusion of the ICP to the process increased the directionality and power enough to apparently etch the anodized aluminum cathode, leaving an outline of the Borofloat sample on the surface. A quartz coverplate was installed onto the face of the cathode to alleviate this problem. Figures 4.14, 4.15, 4.16 and 4.17 are results of this etch process. 
Figure 4.14 is an example of how poor sidewalls and diffraction effects in the PR are transferred to the substrate during the etch process. The material that is located in the trenches is believed to be the nonvolatile byproducts formed from the combinations of the Borofloat dopants sodium, potassium and aluminum with fluorine ions [39].

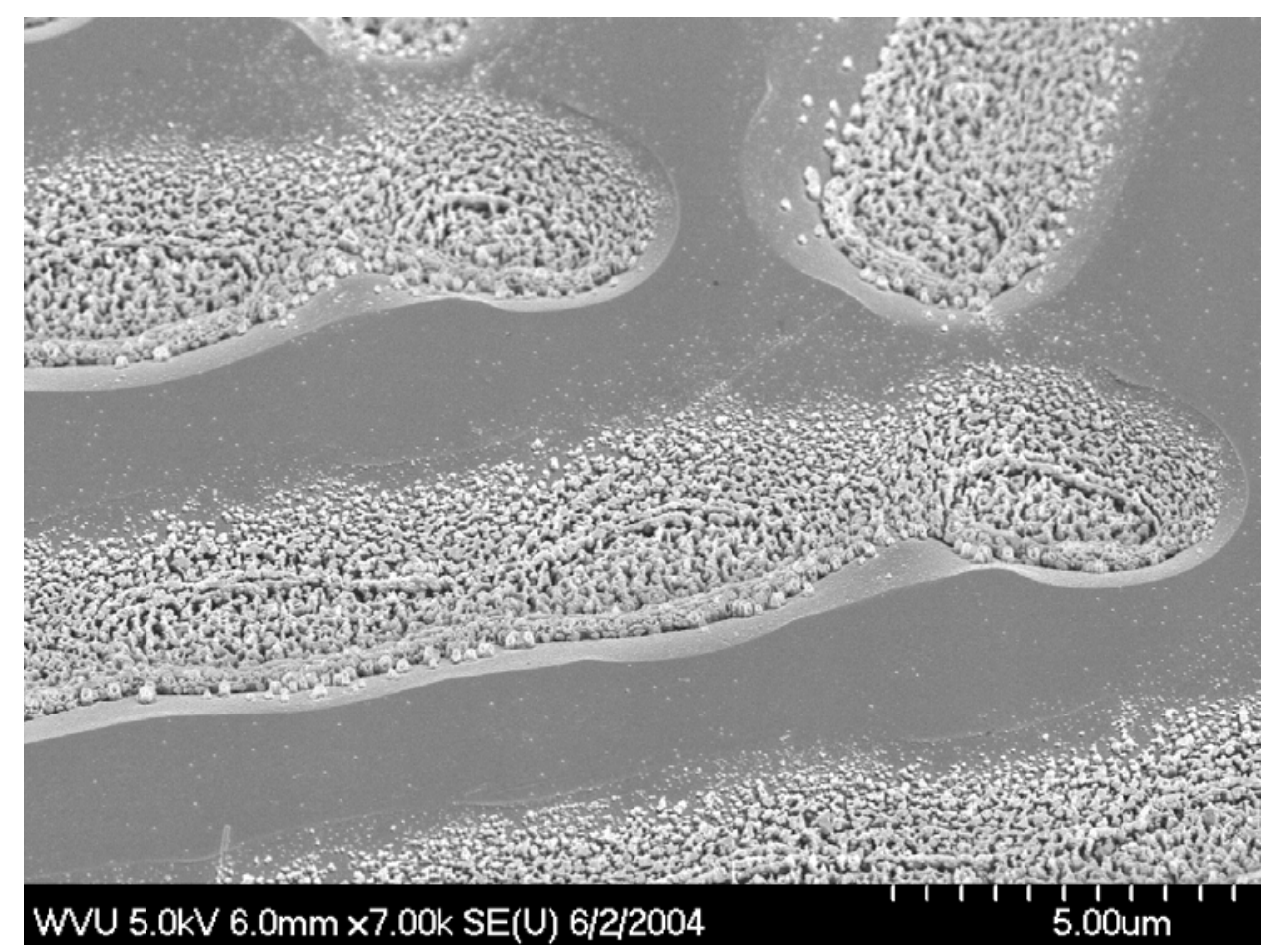

Figure 4.14: Sloped sidewalls and evidence of diffraction effects during photolithography. Debris in middle of trenches is believed to be non-volatile Fluorine compounds.

Figure 4.15 and Figure 4.16 are magnified views of the substrate where it can be seen that the $\mathrm{SiO}_{2}$ and $\mathrm{B}_{2} \mathrm{O}_{3}$ are being removed during the etch process, leaving behind the nonvolatile byproducts. The lower left corner of the Figure 4.15 contains a region that was protected by PR during the RIE and contains what appears to be the tops of the nonvolatile material. This would seem to indicate that the nonvolatile components are already in the Borofloat in the form of the oxides of $\mathrm{Al}$, $\mathrm{Na}$ and $\mathrm{K}$ which may act as "seeds" for growth into larger structures. These "seeds" would not interfere with the optical characteristics of Borofloat due to their small size which appears to be on the order of tens of nanometers as seen in Figure 4.16 


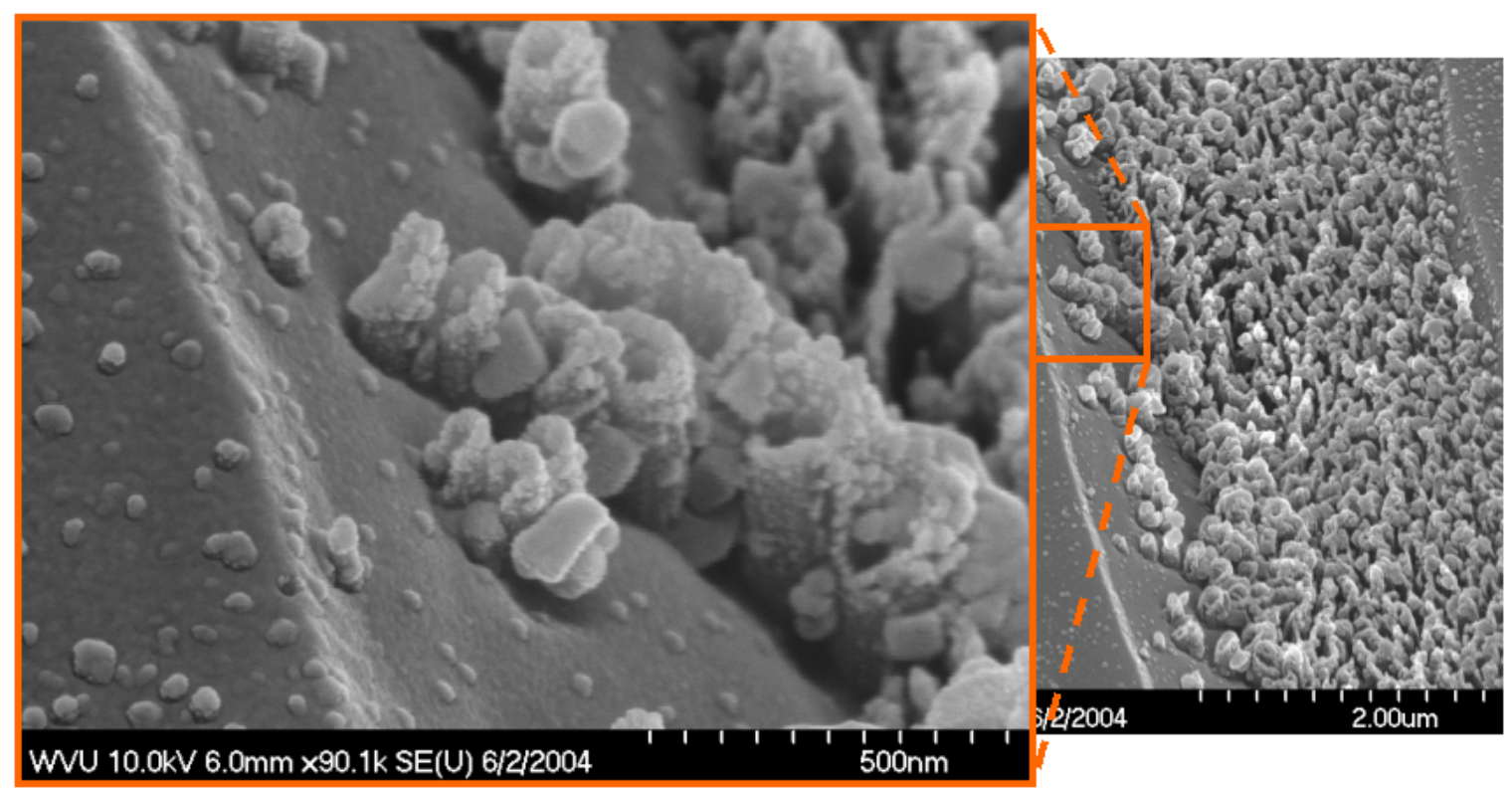

Figure 4.15: Evidence of chemical etching of $\mathrm{SiO}_{2}$ and $\mathrm{B}_{2} \mathrm{O}_{3}$ leaving behind nonvolatile debris.

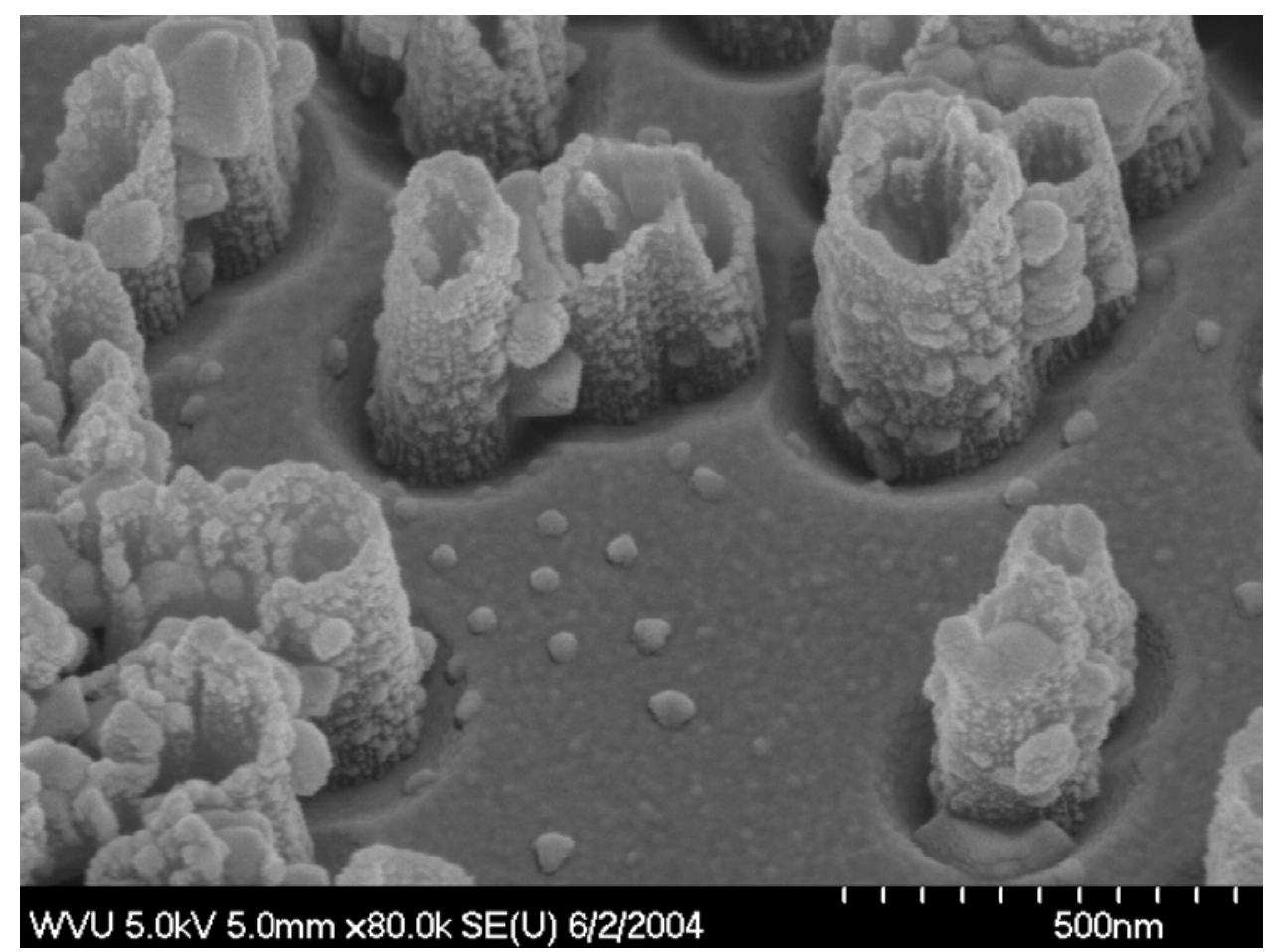

Figure 4.16: Nano-scale Fluorine compound structures.

Once the photolithography was refined with the process found in Table 4.2 used, it was determined that the RIE system would need to be modified to include $\mathrm{SF}_{6}$ to try and 
reduce the flow rate that could be achieved with a stable plasma to move closer to the physical etch. The use of $\mathrm{SF}_{6}$ enabled initiation of a stable plasma with flow rates as low as $8 \mathrm{sccm}$ at a chamber pressure of $9 \mathrm{mT}$. An etch was performed with the following recipe:

\begin{tabular}{|c|c|c|c|c|c|}
\hline Flow Rate & Species & Pressure & RF Power & ICP Power & Etch Time \\
\hline $8 \mathrm{sccm}$ & $\mathrm{SF}_{6}$ & $9 \mathrm{mT}$ & $200 \mathrm{~W}$ & $150 \mathrm{~W}$ & $4 \mathrm{~min}$ \\
\hline
\end{tabular}

The RF power was increased from $140 \mathrm{~W}$ to $200 \mathrm{~W}$ to increase the field between the cathode and the anode of the system. This was to try and compensate for the inability to reach lower operating pressure by increasing the force of impinging ions and radicals with the substrate surface.

The results of this etch are shown in Figure 4.17 which also includes a portion of the L-Edit file for the region being viewed. The vertical sidewalls are attributed to corrections in photolithography and highly anisotropic nature of this etch process while the sloping of the of the $0.5 \mu \mathrm{m}$ tip can be attributed to partial development of PR in this region. Comparison of Figure 4.17 with Figure 4.14 and 4.15 shows a marked erosion of the nonvolatile byproducts by the combination of $\mathrm{SF}_{6}$ and higher $\mathrm{RF}$ power which resulted in an improvement in overall etched surface quality. 


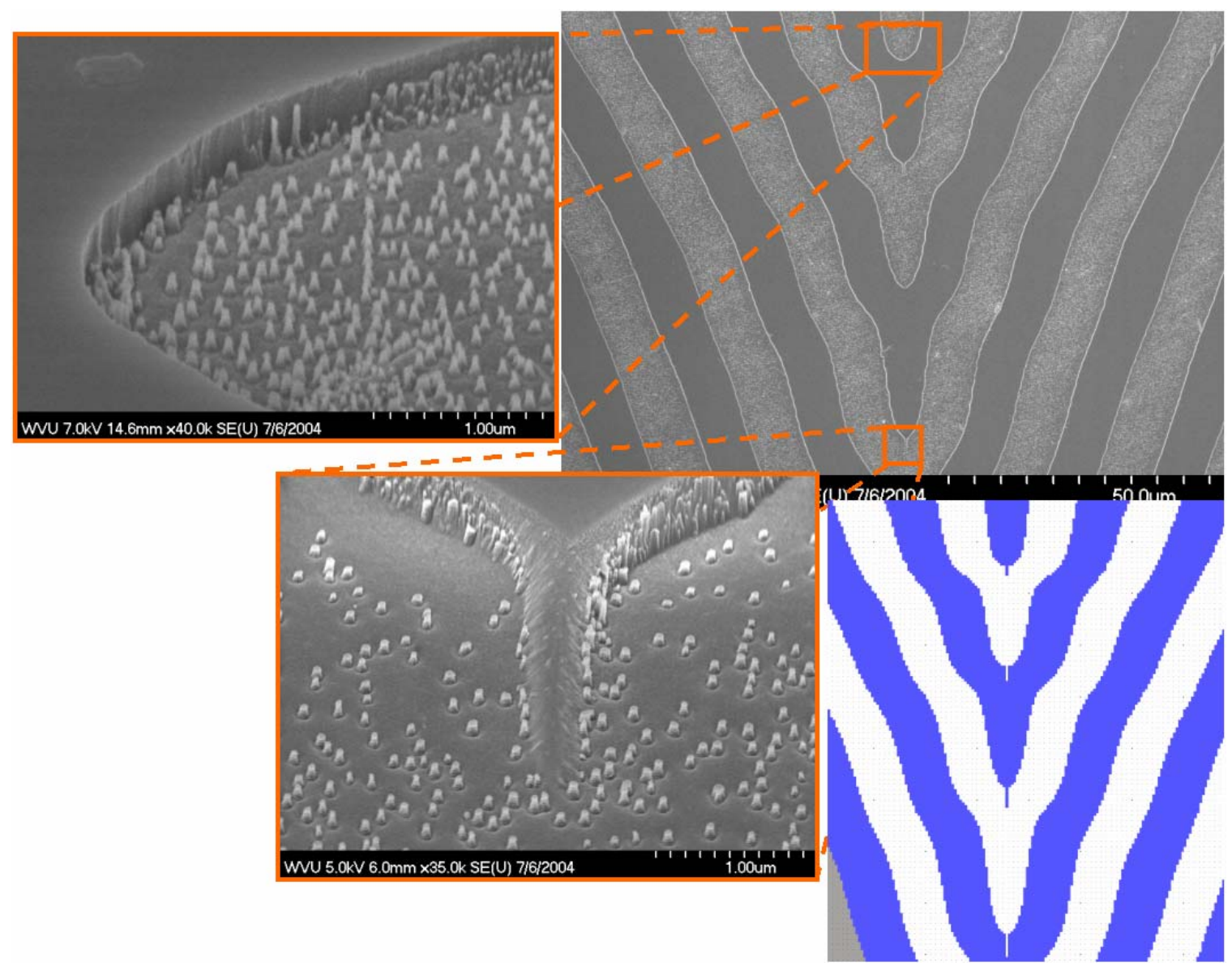

Figure 4.17: Conversion to $\mathrm{SF}_{6}$ process gas and modified RIE recipe shows marked improvement in sidewalls and removal of debris.

\subsection{Bulk Optical Tests of Lens Design}

Optical testing of the DOEs was performed using the Spiricon LBA-710PC 10-bit analog laser beam analyzer with COHU 4812 monochrome frame transfer Charge Coupled Device (CCD) camera. The lens patterns, initially photoresist on Borofloat substrate and then etched Borofloat only, were tested using an unpolarized $633 \mathrm{~nm}$ wavelength Helium-Neon (HeNe) laser to illuminate the individual DOEs. The output was then magnified using a 10x Leitz microscope objective to overcome the combination of the desired $10 \mu \mathrm{m}$ diameter output beam and the CCD pixel size of $11.5 \mu \mathrm{m}(\mathrm{H})$ by 27 $\mu \mathrm{m}(\mathrm{V})$ [40]. The output of the DOE was then sent through an array of Neutral Density (ND) filters to reduce the amount of power incident on the CCD camera to prevent damage. The percent transmission data of the ND filters is shown in Table 4.3 [41] 


\begin{tabular}{|l|l|}
\hline ND VALUE & TRANSMI SSI ON \\
\hline 0.3 & $50 \%$ \\
\hline 0.7 & $20 \%$ \\
\hline 1.0 & $10 \%$ \\
\hline 2.0 & $1 \%$ \\
\hline 3.0 & $0.1 \%$ \\
\hline 4.0 & $0.01 \%$ \\
\hline
\end{tabular}

Table 4.3: Spiricon ND filter transmission values [41].

\section{Power Calibration}

Power calibration of the Spiricon system to the incident beam required an independent power measurement of the beam prior to data collection. United Detector Technology's radiometer/photometer model 350 with a detector area of $0.20 \mathrm{~cm}^{2}$ [42] was used to sample the HeNe beam at various points throughout the optical path, primarily at interfaces as seen in Figure 4.18, to ensure proper calibration of the Spiricon detector.

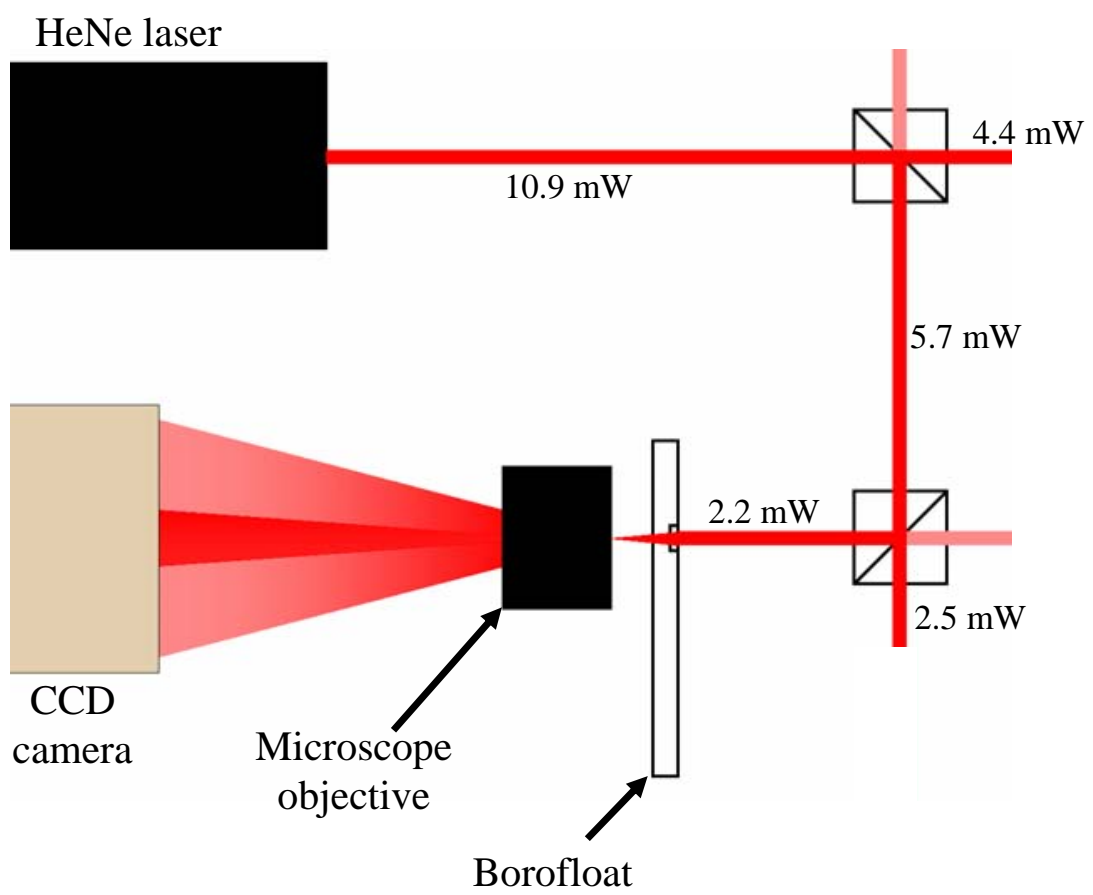

Figure 4.18: Schematic of experimental setup. 
Output power of the laser was found to be $10.9 \mathrm{~mW}$ which was reduced to $2.2 \mathrm{~mW}$ at the Borofloat/air interface through the use of beamsplitters which directed the beam to the sample. The power was reduced to $2 \mathrm{~mW}$ and $1.9 \mathrm{~mW}$ after transmission through an open area of the Borofloat substrate and transmission through a DOE respectively. This reduction is due to the approximately $90 \%$ transmission characteristics of the substrate at this wavelength as seen in Figures 4.4 and 4.5. The power measured after transmission through the Leitz objective was found to be $1.9 \mathrm{~mW}$ through open Borofloat and $1.6 \mathrm{~mW}$ through a DOE. Measurements were then taken at the front of the Spiricon laser beam sampler (LBS) used to hold the ND filters and the front of the COHU camera and found to be approximately $420 \mathrm{nW}$ at both locations. A power recalibration was required prior to testing of each DOE from changes in power distributions and location of the input beam to the DOE. The power measurements were taken in front of the LBS and then multiplied by the transmission values of the ND filters used. The amount of power that was focused by individual DOEs and the saturation of the COHU camera determined the number and value of the ND filters used.

\section{Beam Measurements}

Measurements were taken using DOEs in rows one through four of columns three and five which were designed for a focal length of $5 \mathrm{~mm}$ and wavelengths of $633 \mathrm{~nm}$ and $1310 \mathrm{~nm}$ respectively. The magnification of the output beam was determined by imaging the lettering in the row and column labels of the DOE grid and measuring the size at the location of the COHU camera after being brought into focus using the microscope objective. The amount of magnification is calculated by dividing the measured image by the letter width of $56 \mu \mathrm{m}$. The image captured by the CCD camera of a portion of the letter "L" is shown in Figure 4.19. The measured width of the imaged letter is approximately $1.998 \mathrm{~mm}$ if the centers of lowest intensity are chosen as the letter edges corresponding to a magnification of 35.7 which is used to calculate output diameters shown in Table 4.5. 


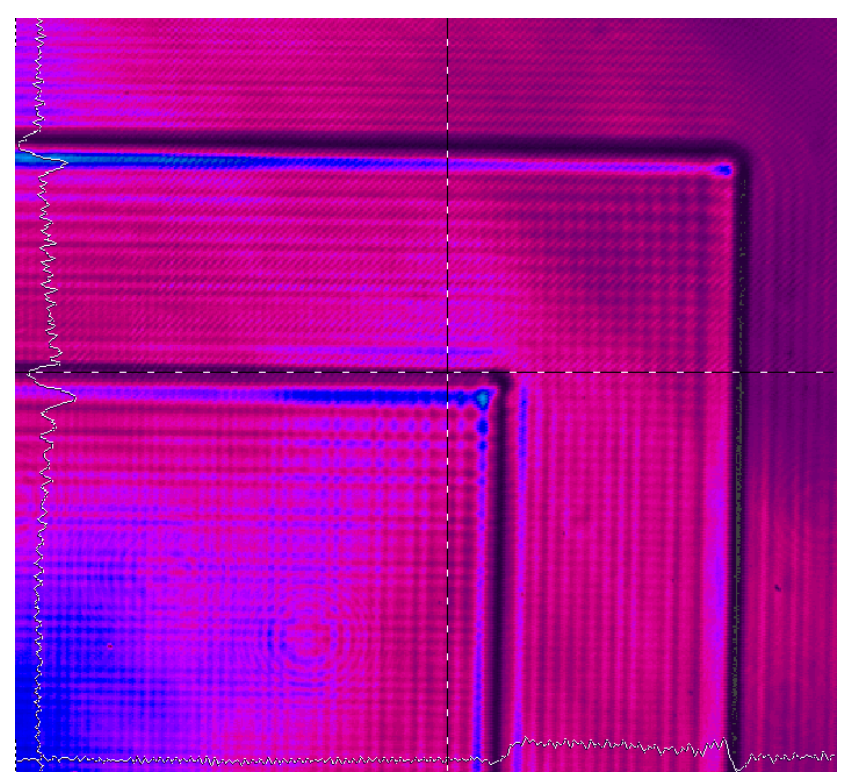

Figure 4.19: Portion of the letter "L" used to determine amount of magnification from optics system.

Calculation of output beam diameters from experimental data was performed using $1 / \mathrm{e}^{2}$ as a cutoff of the peak energy detected. The raw measurements from the Spiricon software are shown in Table 4.4 while Table 4.5 contains the calculated beam size taking into account the magnification of the objective lens. The left hand column of Tables 4.4, 4.5, and 4.6 give the location of the beam being tested and region that was measured. For example, $C 3 R 1 a B$ refers to column 3, row 1 , and bottom intensity. 


\begin{tabular}{lrrrr}
\multicolumn{5}{c}{ Raw Data } \\
C3R1B & Peak (nW/cm^2) & WidthX (um) & WidthY (um) & $\begin{array}{c}\text { Calculated } \\
\text { Diameter (um) }\end{array}$ \\
C3R1T & $8.520 \mathrm{E}+02$ & $2.295 \mathrm{E}+02$ & $3.375 \mathrm{E}+02$ & $2.835 \mathrm{E}+02$ \\
C3R2L & $2.023 \mathrm{E}+02$ & $2.295 \mathrm{E}+02$ & $5.535 \mathrm{E}+02$ & $3.915 \mathrm{E}+02$ \\
C3R2R & $6.633 \mathrm{E}+02$ & $3.780 \mathrm{E}+02$ & $2.565 \mathrm{E}+02$ & $3.172 \mathrm{E}+02$ \\
C3R3 & $6.443 \mathrm{E}+02$ & $3.915 \mathrm{E}+02$ & $2.700 \mathrm{E}+02$ & $3.308 \mathrm{E}+02$ \\
C3R4L & $7.131 \mathrm{E}+02$ & $2.295 \mathrm{E}+02$ & $3.375 \mathrm{E}+02$ & $2.835 \mathrm{E}+02$ \\
C3R4R & $7.953 \mathrm{E}+02$ & $2.565 \mathrm{E}+02$ & $3.510 \mathrm{E}+02$ & $3.038 \mathrm{E}+02$ \\
& $8.184 \mathrm{E}+02$ & $2.430 \mathrm{E}+02$ & $3.510 \mathrm{E}+02$ & $2.970 \mathrm{E}+02$ \\
C5R1 & & & & \\
C5R2L & $6.442 \mathrm{E}+02$ & $4.590 \mathrm{E}+02$ & $4.590 \mathrm{E}+02$ & $4.590 \mathrm{E}+02$ \\
C5R2R & $4.850 \mathrm{E}+02$ & $4.860 \mathrm{E}+02$ & $4.590 \mathrm{E}+02$ & $4.725 \mathrm{E}+02$ \\
C5R3 & $4.841 \mathrm{E}+02$ & $6.615 \mathrm{E}+02$ & $4.455 \mathrm{E}+02$ & $5.535 \mathrm{E}+02$ \\
C5R4L & $6.649 \mathrm{E}+02$ & $4.860 \mathrm{E}+02$ & $6.750 \mathrm{E}+02$ & $5.805 \mathrm{E}+02$ \\
C5R4R & $2.212 \mathrm{E}+02$ & $5.130 \mathrm{E}+02$ & $6.750 \mathrm{E}+02$ & $5.940 \mathrm{E}+02$ \\
& $2.198 \mathrm{E}+02$ & $5.535 \mathrm{E}+02$ & $6.750 \mathrm{E}+02$ & $6.142 \mathrm{E}+02$
\end{tabular}

Table 4.4: Peak power and beam size.

$\begin{array}{lccc}\text { WidthX (um) } & \text { WidthY (um) } & \begin{array}{c}\text { Calculated } \\ \text { Diameter (um) }\end{array} \\ \text { C3R1B } & 6.43 & 9.45 & 7.94 \\ \text { C3R1T } & 6.43 & 15.50 & 10.97 \\ \text { C3R2L } & 10.59 & 7.18 & 8.89 \\ \text { C3R2R } & 10.97 & 7.56 & 9.27 \\ \text { C3R3 } & 6.43 & 9.45 & 7.94 \\ \text { C3R4L } & 7.18 & 9.83 & 8.51 \\ \text { C3R4R } & 6.81 & 9.83 & 8.32 \\ & & & \\ \text { C5R1 } & 12.86 & 12.86 & 12.86 \\ \text { C5R2L } & 13.61 & 12.86 & 13.24 \\ \text { C5R2R } & 18.53 & 12.48 & 15.50 \\ \text { C5R3 } & 13.61 & 18.91 & 16.26 \\ \text { C5R4L } & 14.37 & 18.91 & 16.64 \\ \text { C5R4R } & 15.50 & 18.91 & 17.20\end{array}$

Table 4.5: Approximate output beam sizes.

The larger beam widths of the $1310 \mathrm{~nm}$ relative to the $633 \mathrm{~nm}$ DOEs can be attributed to the $633 \mathrm{~nm}$ input wavelength of the HeNe laser used during testing. Table 4.6 is the 
percent error between the measured output beam diameters and the desired theoretical output beam diameters of $10 \mu \mathrm{m}$.

\begin{tabular}{lrrr} 
& \multicolumn{3}{c}{ Percent Error in Beam size } \\
C3R1B & WidthX (um) & WidthY (um) & Diameter (um) \\
C3R1T & -35.71 & -5.46 & -20.59 \\
C3R2L & -35.71 & 55.04 & 9.66 \\
C3R2R & 5.88 & -28.15 & -11.15 \\
C3R3 & 9.66 & -24.37 & -7.34 \\
C3R4L & -35.71 & -5.46 & -20.59 \\
C3R4R & -28.15 & -1.68 & -14.90 \\
& -31.93 & -1.68 & -16.81 \\
C5R1 & & & \\
C5R2L & 28.57 & 28.57 & 28.57 \\
C5R2R & 36.13 & 28.57 & 32.35 \\
C5R3 & 85.29 & 24.79 & 55.04 \\
C5R4L & 36.13 & 89.08 & 62.61 \\
C5R4R & 43.70 & 89.08 & 66.39 \\
& 55.04 & 89.08 & 72.04
\end{tabular}

Table 4.6: Percent error of experimental DOE focal beam size vs. desired size of $10 \mu \mathrm{m}$.

The negative values in the Table 4.6 represent beam measurements that are smaller than the desired probe diameter of $10 \mu \mathrm{m}$. The much larger variations in beam width between the visible and IR DOEs can be attributed to the use of the shorter wavelength $633 \mathrm{~nm}$ laser as the input beam.

These variations from the expected $10 \mu \mathrm{m}$ size can also be partially explained by the calculation methods used by the Spiricon software. The user can draw an aperture on the beam display to window out extraneous noise and focus on a region of interest. Depending on the computation method employed, in this case percent of peak power with a cutoff power of $13.5 \%$ corresponding to $1 / \mathrm{e}^{2}$, the beam diameter is measured. A graphical display of the measured beam area is displayed to help the user ensure a reasonable area is included but can lead to beam area that are distorted by secondary peaks located too closely to the apertured area as seen in Figure 4.20. The white circle is the drawn aperture and the black ellipse inside is the calculated beam. 


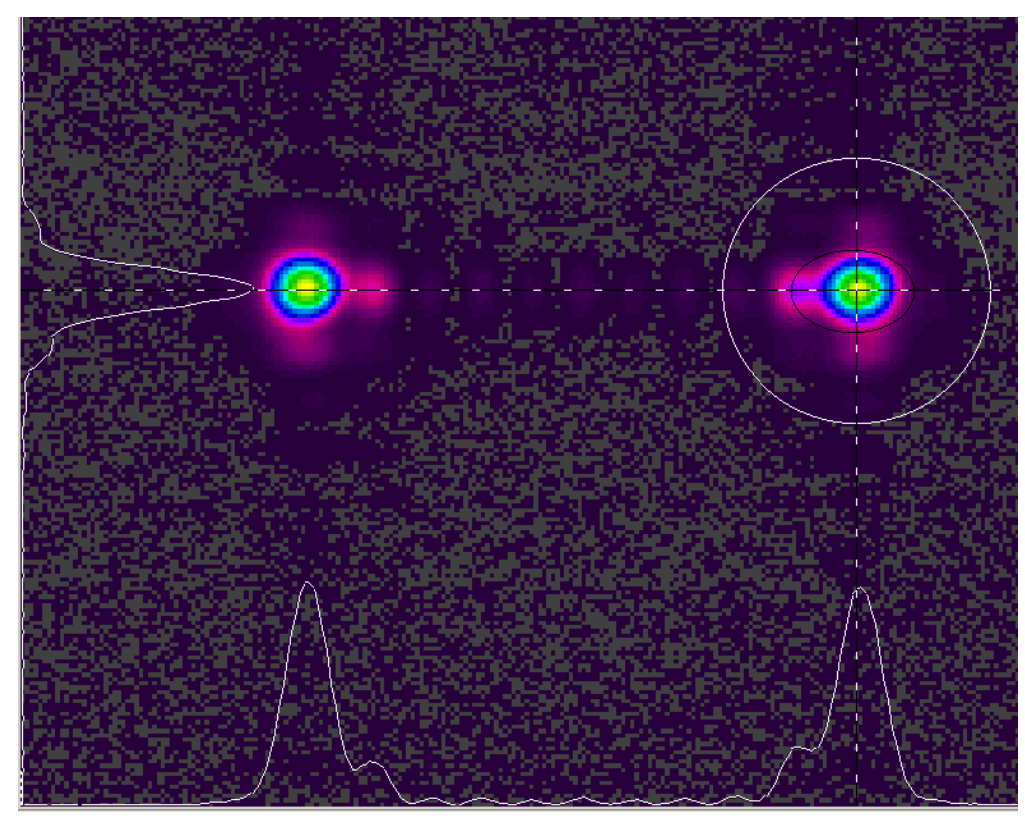

Figure 4.20: Experimental measurement of output from C3R2.

The assumption that the beam incident on the DOEs would be perfectly Gaussian can also lead to discrepancies between output beam radii calculated in the MATLAB simulations and the actual beam radii from experimental results. The laser used for experimental work may not consistently generate a beam with a Gaussian profile due to defects or environmental conditions. Another cause for deviation could be the interaction with optical components that direct the beam from the laser to the DOE, primarily the beamsplitters used in the experimental setup. The profile of the input beam shown in Figure 4.21 has been passed through beamsplitters used in the optical setup and displays a definitely un-Gaussian profile. Figure 4.22 is a profile of the input beam that has not been routed through other optical components, exhibiting a slightly more Gaussian shape. 


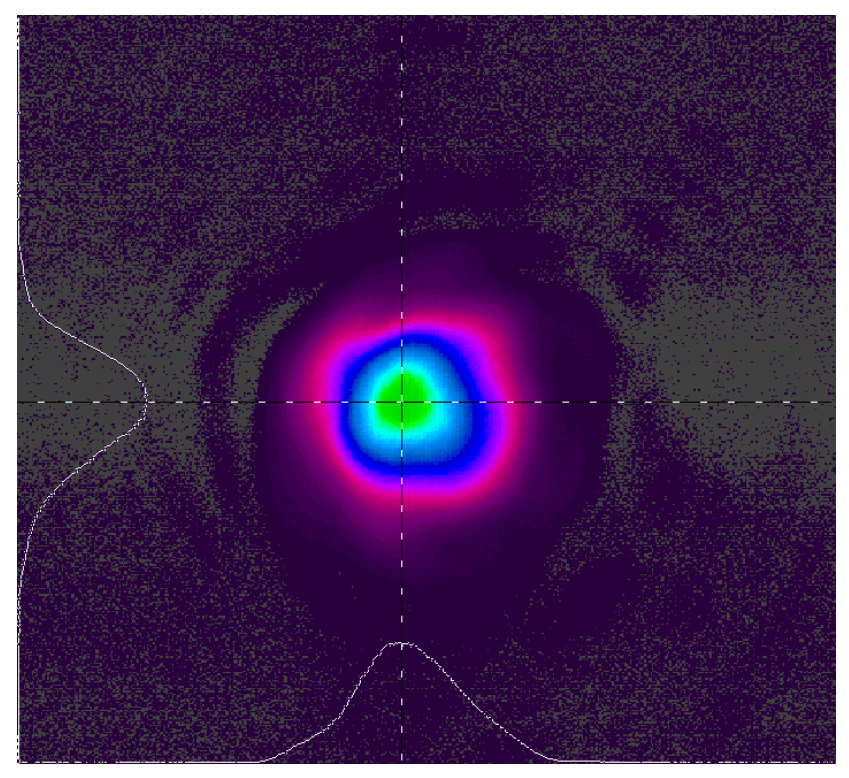

Figure 4.21: Input beam routed through two beamsplitters.

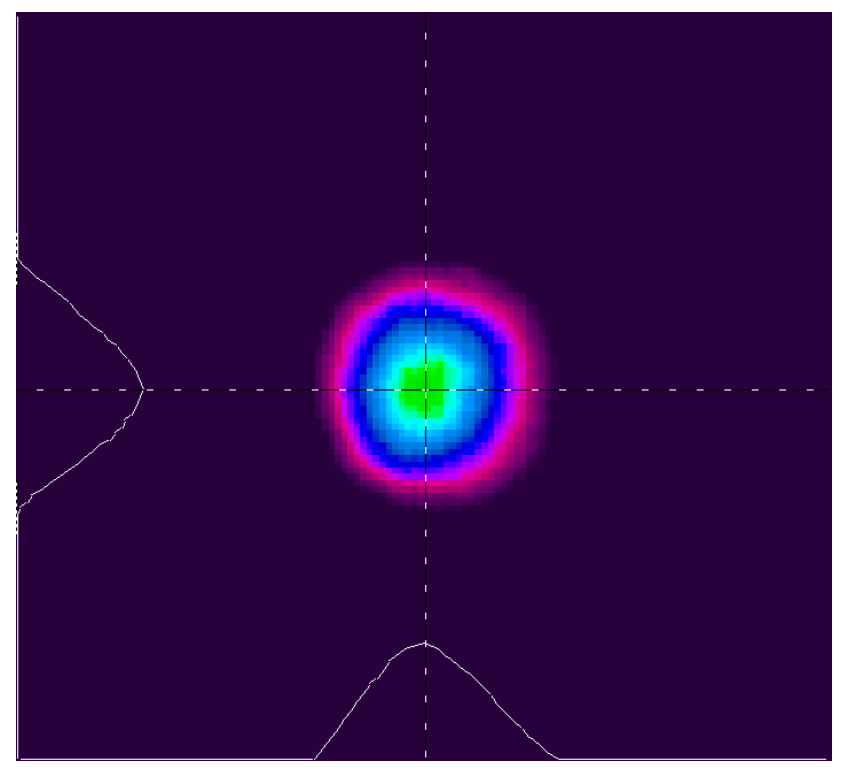

Figure 4.22: Input beam without intervening optical components.

Although the measured output beam diameters appear to deviate from the desired 10 $\mu \mathrm{m}$ diameter, comparison of the experimental output from lenses such as C3R2 show good correspondence in shape and relative size with the simulated output as shown in Figures 4.23 and 4.24 . 


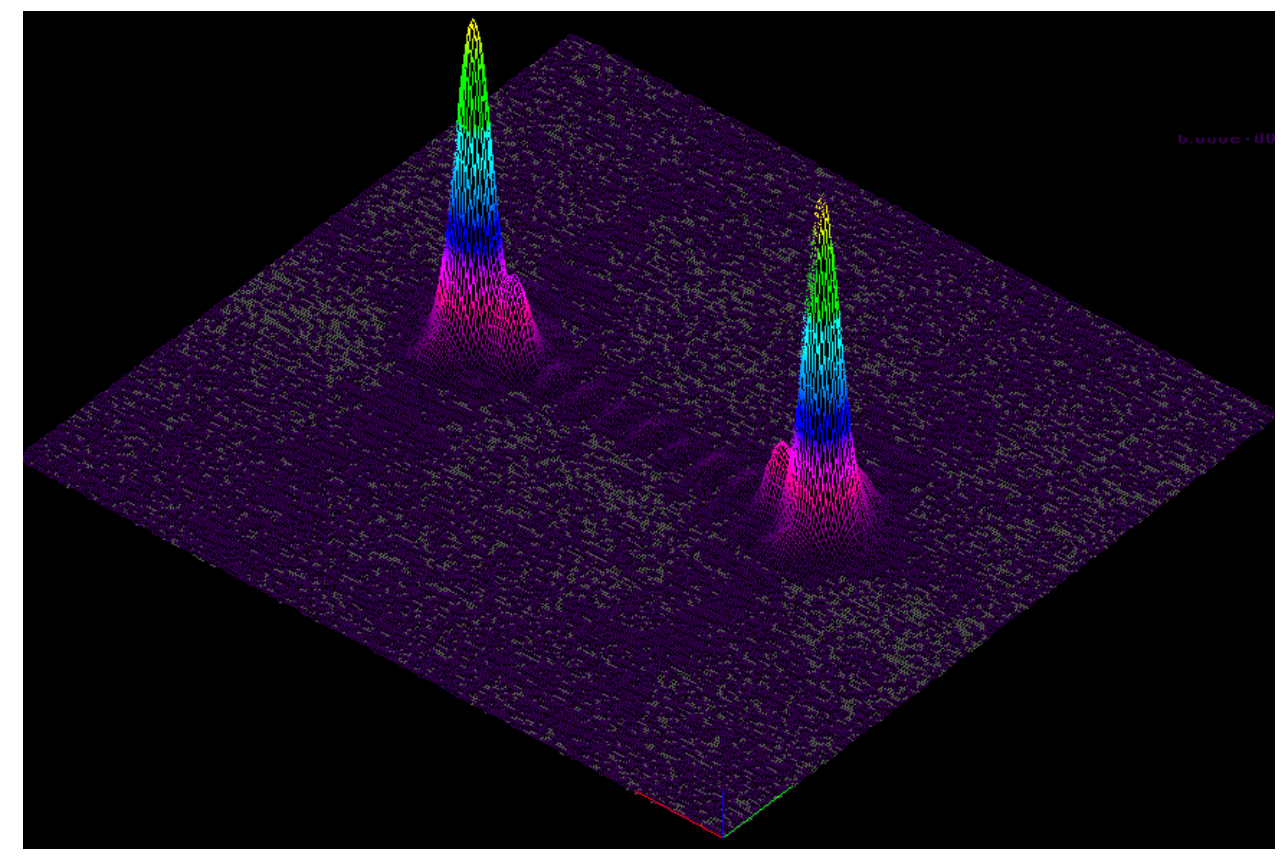

Figure 4.23: 3D experimental output from C3R2.

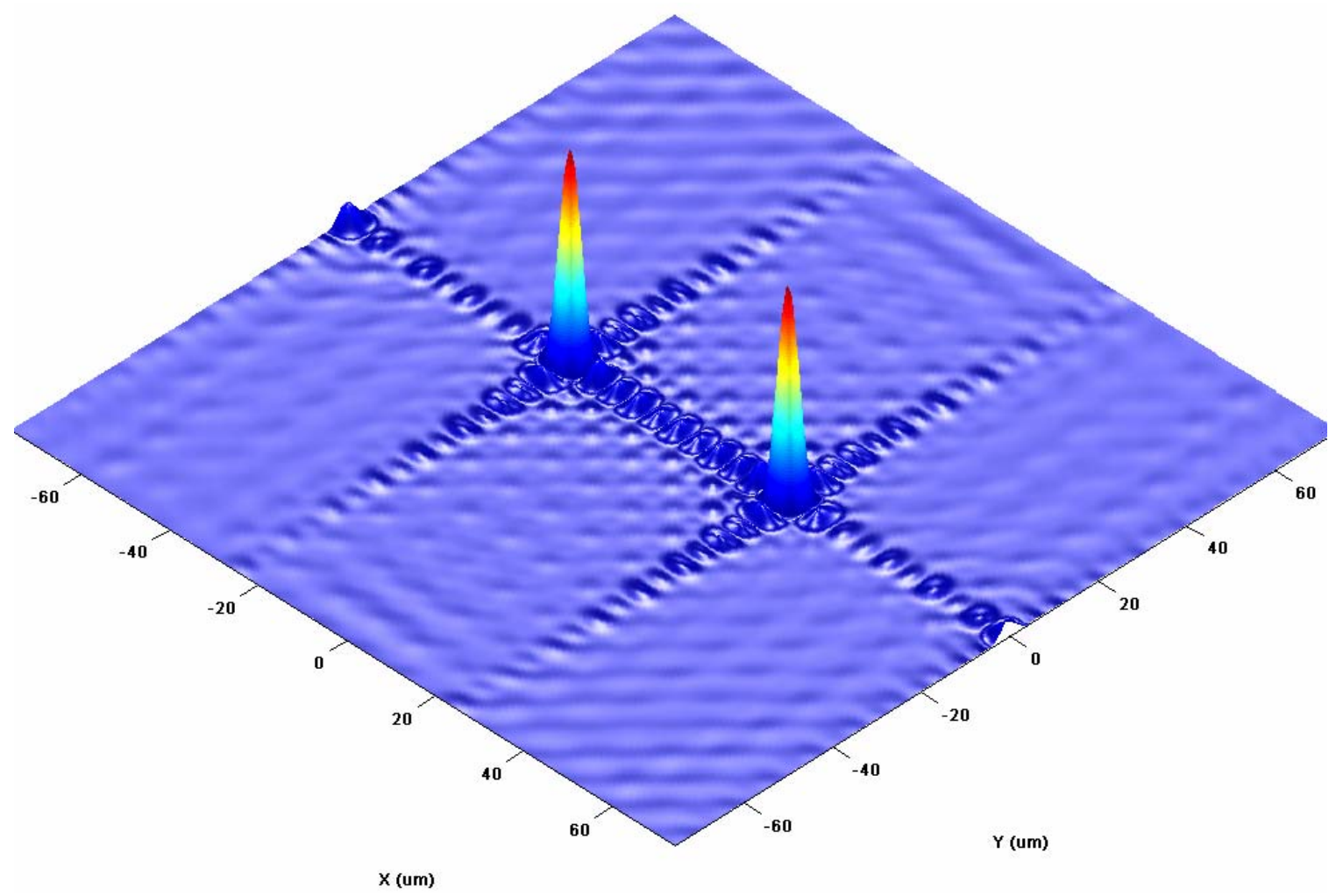

Figure 4.24: 3D simulated output from C3R2. 
The entire simulated output region of C3R4 is shown in Figure 4.26 followed by a photograph of the experimental output in Figure 4.27. The photograph was taken to illustrate regions of the output plane that could not simulated due to memory requirements as well as regions that could not be easily seen with the COHU camera. The vertical banding and secondary peaks that appeared during simulation can be seen in the experimental output as well as the outline of the DOE itself. The appearance of a mirrored beam pattern in regions outside the DOE, at the bottom of the photo, lends credence to the hypothesis that the repetition of the DOE pattern, as seen in Figure 4.25, are a corrective factor to reduce comatic aberrations in the desired output.

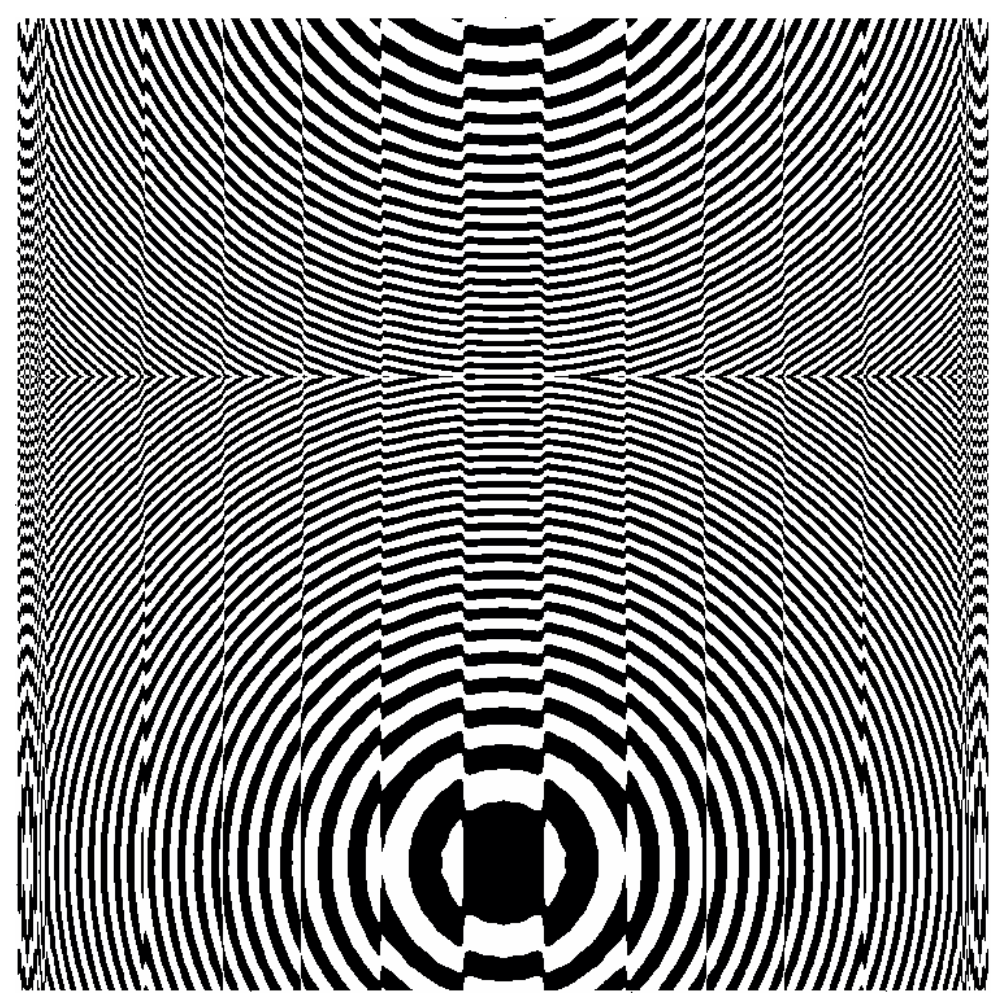

Figure 4.25: Generated DOE profile for C3R4 


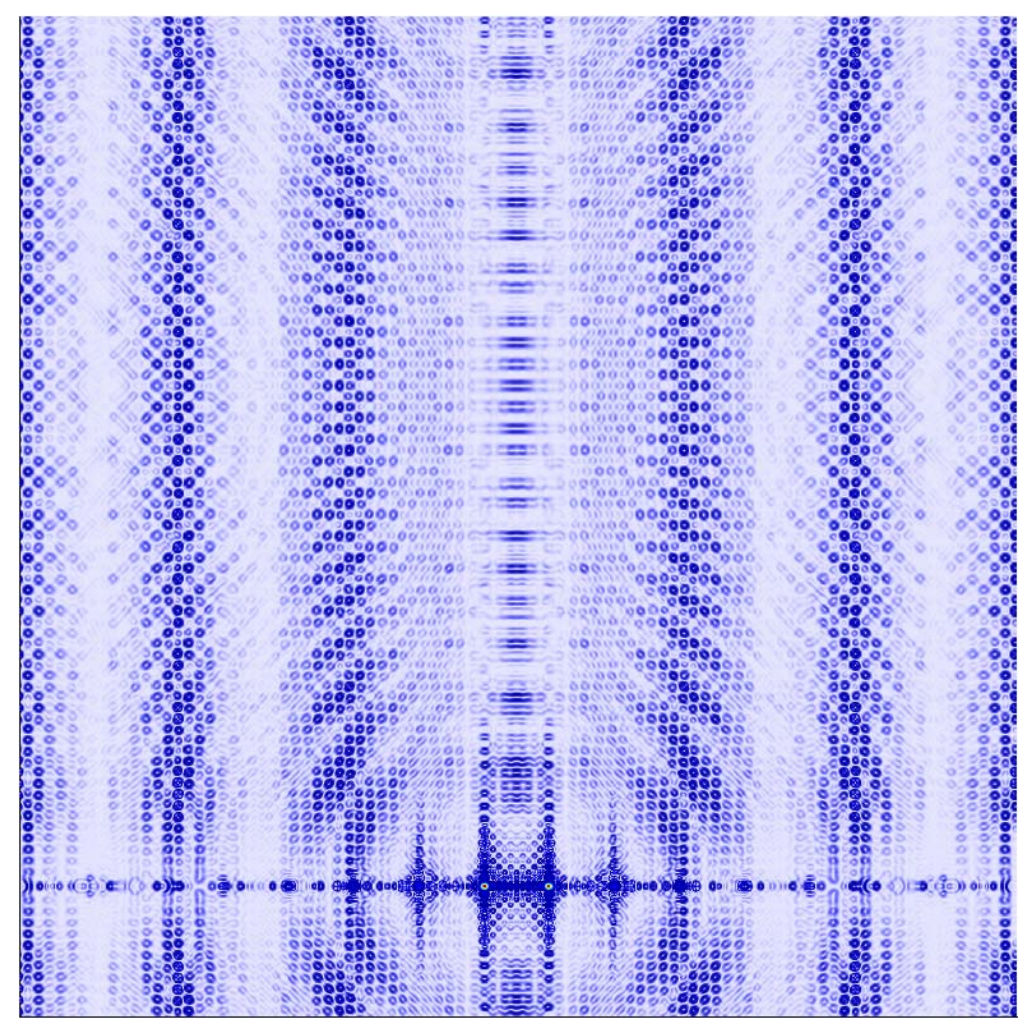

Figure 4.26: Simulated output of C3R4.

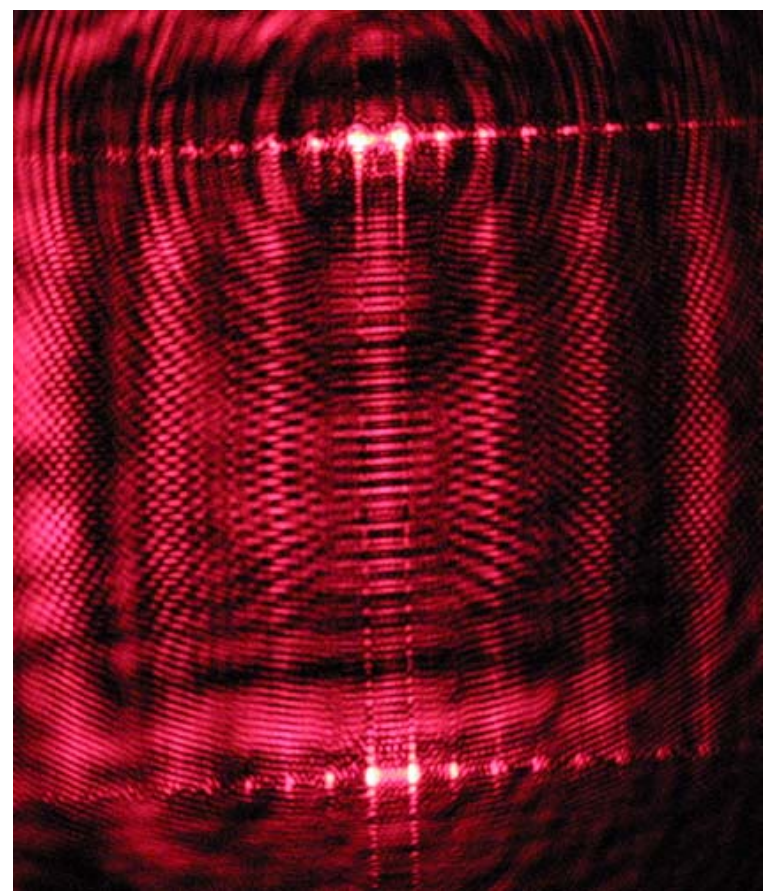

Figure 4.27: Photograph of experimental output from C3R4. 
The desired focal points in the experimental output, located at the top of Figure 4.27, formed nearly circular focal points even though off-axis while the mirror image outside the region of the DOE suffered comatic aberration as seen in Figures 4.28 and 4.29. This shows that some of the beam energy is being diffracted away from the region of interest to reduce comatic aberration.

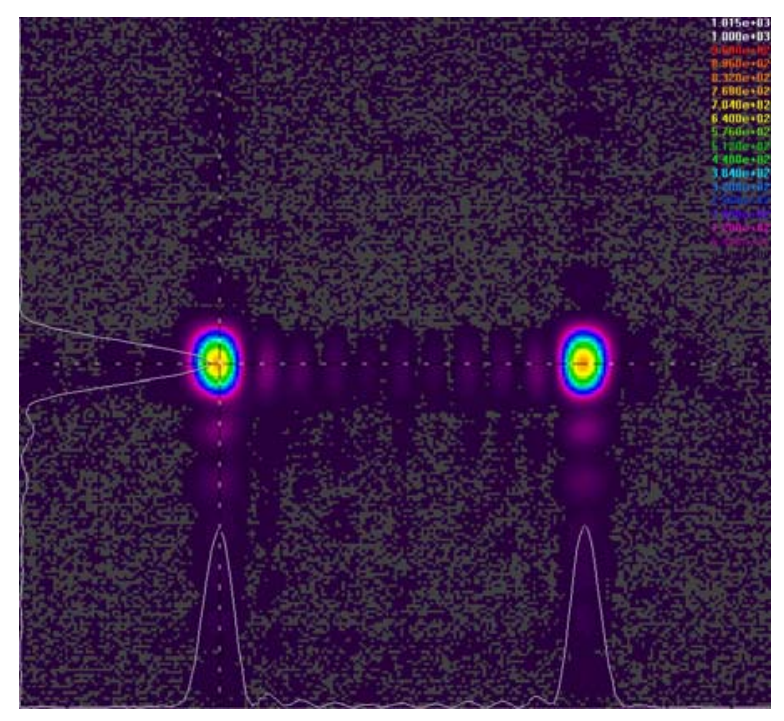

Figure 4.28: Desired focal points in the off-axis case.

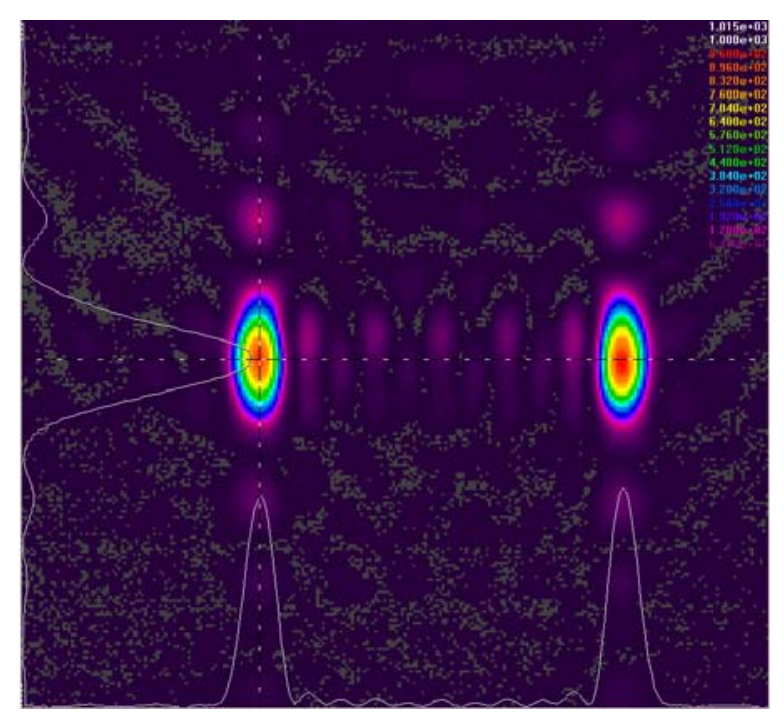

Figure 4.29: Mirrored region outside of DOE. 
Further validation of the simulation tool can be found in the improper removal of noise regions from the DOE used for location C3R1. A section of the DOE approximately $85 \mu \mathrm{m}$ above the center was removed instead of regions on the perimeter. This led to a DOE that resembled a slightly misshapen on-axis Fresnel zone plate as seen in Figure 4.30.

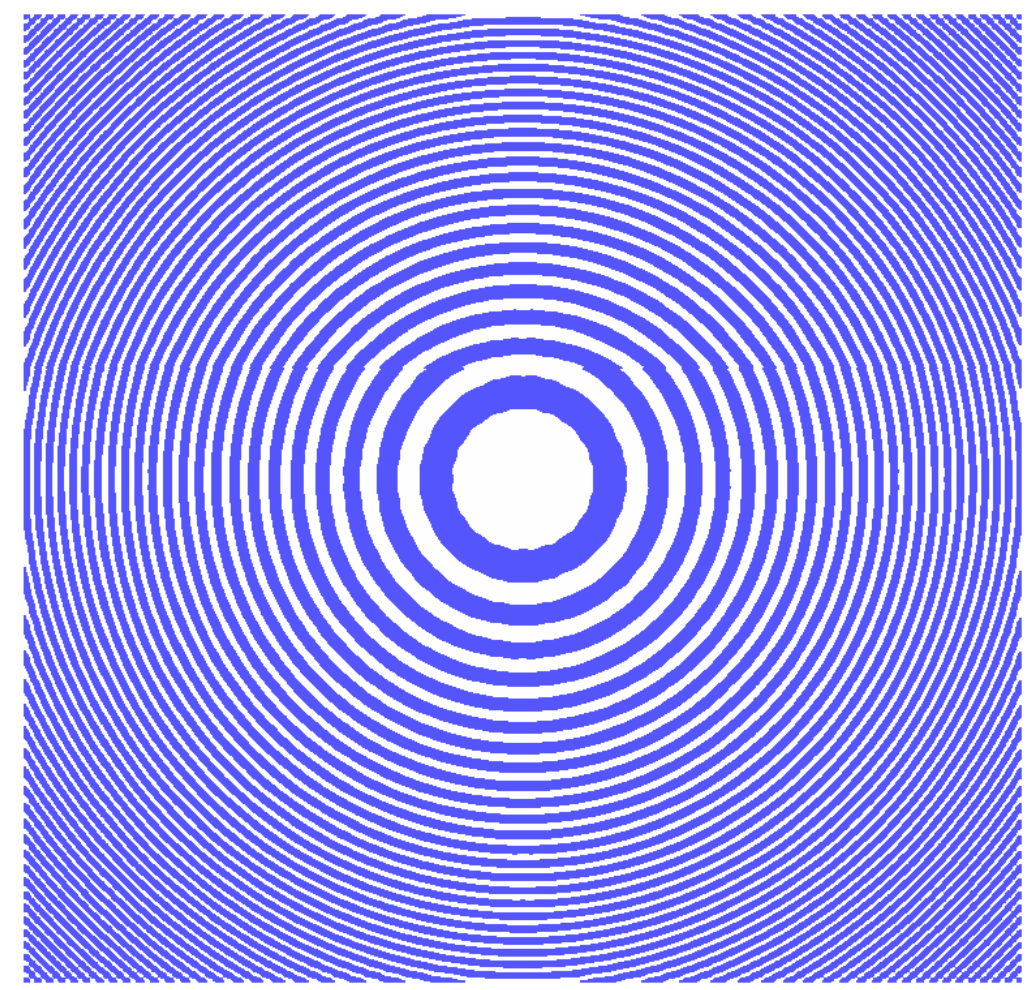

Figure 4.30: L-Edit layout of incorrect on-axis single probe DOE.

The simulated output from this DOE displayed a secondary peak as shown in Figure 4.31 and was confirmed with the experimental output shown in Figure 4.32. The secondary peak in the output beam also contributed to the large disparity in beam size found in Table 4.6 for this DOE. 


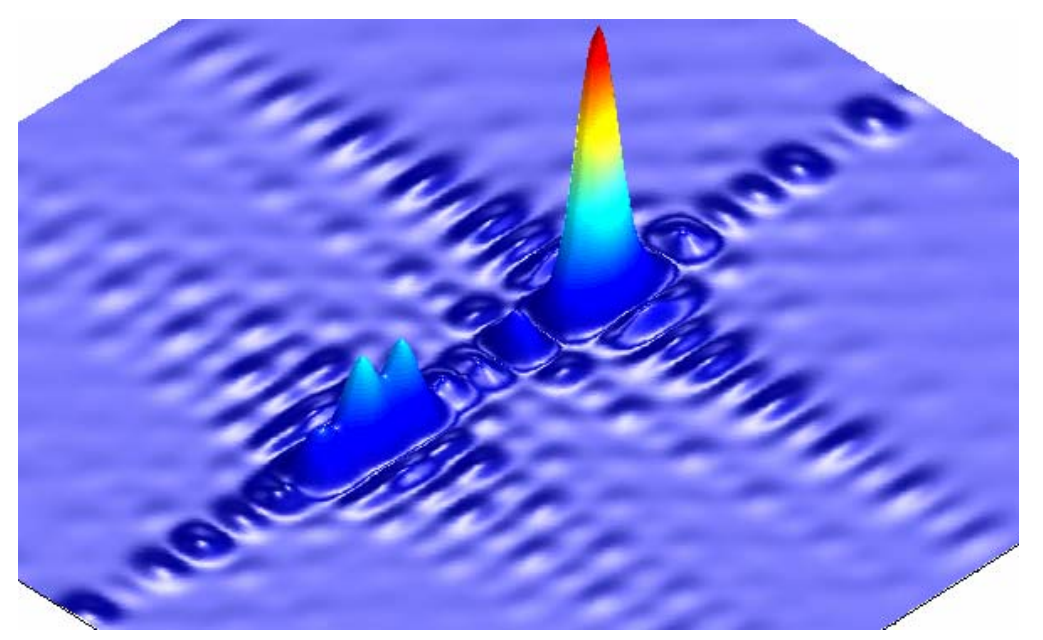

Figure 4.31: Magnified view of central output intensity of incorrect DOE.

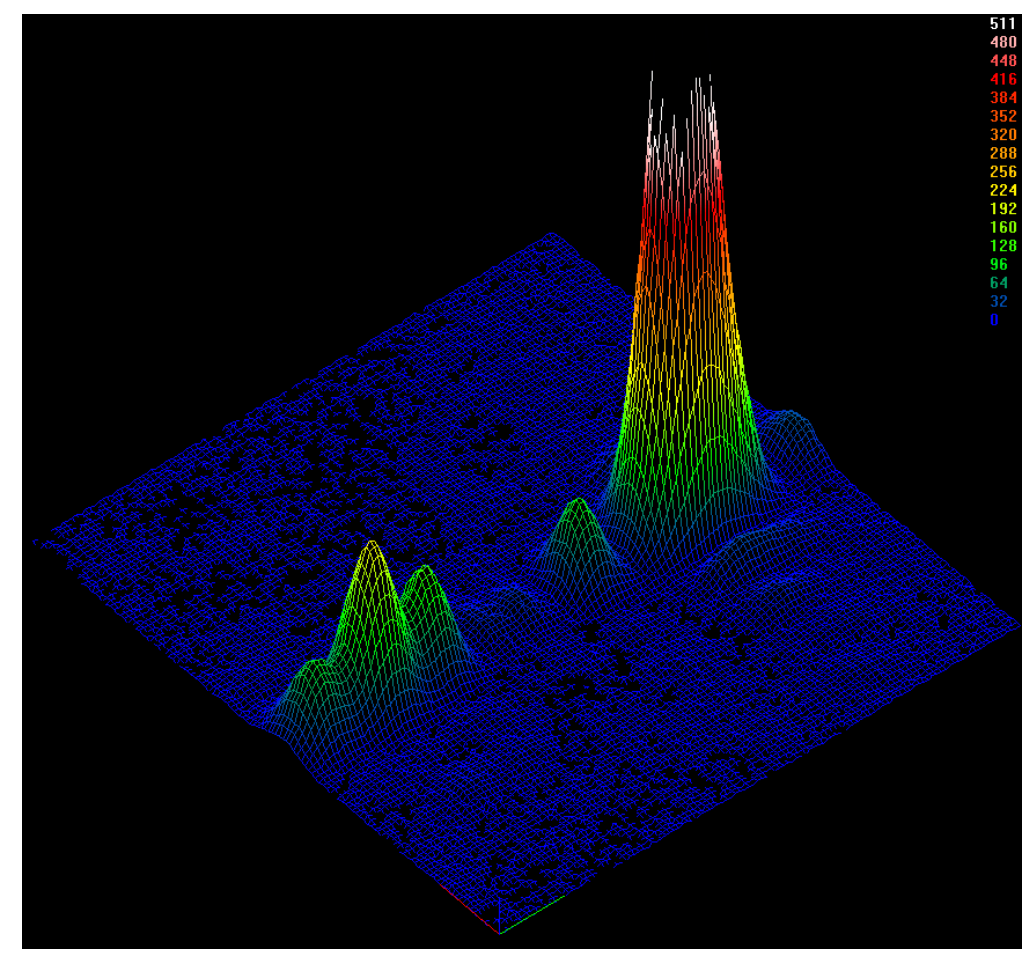

Figure 4.32: Experimental data from incorrect DOE patterned in PR. Notice correlation with simulation output shown in Figure 4.31. 


\section{Chapter 5: Conclusion and Future Investigation}

This thesis research established tools and processes for the design, fabrication and characterization of diffractive optical elements (DOEs) for achieving IOM in MEMS. DOEs for on and off-axis single and multiple optical probe beam generation were successfully modeled using on a simulation tool developed based on linear optical system model paraxial gaussian beam theory. The DOE pattern and subsequent masks for fabrication were generated using this MATLAB tool to calculate the desired pattern based on knowledge of the input wave and the desired optical probe beam output pattern. DOEs were successfully fabricated on a borosilicate substrate and characterized using a beam profiler to determine the number, shape and power of the probe beams for comparison with the results from the simulation tool. Experimental DOE imaging results compare well with those obtained from the modeling tool validating the DOE design tool and the fabrication process.

A number of areas arose during the course of this research which bear further investigation. Among these, were those associated with the use of Matlab on the Windows PC platform. Some of the problems with using Windows operating system and MatLab to run these lens simulations include memory issues [43]. Apparently, a 32-bit 
architecture is limited to a maximum of 4 GB of virtual memory regardless of the amount of ram installed due to the limitation of $2^{32}$ possible memory addresses. This is further reduced to 2 GB by the Windows operating system due to design decision to reserve address area for Windows operating system use. This reduction can be decreased to 1 GB by using either Windows 2000 Advanced Server or Windows 2000 Datacenter Server. In addition, the user will also lose another 0.5 GB launching the MATLAB program.

In the critical area of lens fabrication, achieving an optically flat etch surface remains an area where further improvement can be achieved. The fabrication process could be improved using an RIE species, such as argon, to move to a full physical etch. Such a process would have to ensure the PR layer is thick enough to survive the etch to reach the depth required by the process due the lack of selectivity between materials.

The simulation tools created in Matlab could be enhanced to reduce simulation time by modifying the off-axis output plane generation to only calculate half of the beam and then use symmetry to complete. The use of symmetry could also be applied to the calculation of the input by performing matrix manipulations similar to those employed with the on-axis output beam. The would allow the user to only calculate a quarter of the input beam as long as it was simulated on the optical axis. The possibility of converting the simulation tools to a programming language such as $\mathrm{C}++$ that does not require contiguous memory blocks for calculation could also be explored. The DOEs generated by these tools could also be used as starting profiles for optimization techniques such as simulated annealing, finite difference time domain methods, or genetic algorithms. One example of an optimization using these techniques could be the reduction of background noise in the output plane 


\title{
Appendix A: Lens Design Programs
}

\author{
A.1 CreateLens.m \\ \%\%\%\%\%\%\%\%\%\%\%\%\%\%\%\%\%\%\%\%\%\%\%\%\%\%\%\%\%\%\%\%\%\%\%\%\%\%\%\%\%\%\%\%\%\%\%\%\%\%\%\%\%\%\%\%\%\%\%\%\%\%\%\%\%\%\%\%\%\% \\ \% CREATELENS.m $m \%$ \\ \% Uses separate functions to calculate the following: 2-D complex input and output \% \\ $\%$ Gaussian Beams, Fresnel approximation of the transfer function of free space and \% \\ $\%$ save these arrays to files uin.mat, bp.mat, uoutmain.mat, hhxy.mat respectively. \% \\ $\%$ other functions will then modify uoutmain to generate multiple spots if desired. \% \\ $\%$ uoutmain and hhxy are then converted to the spatial frequency domain and \% \\ $\%$ multiplied together and then converted back to the spatial domain to compute the \% \\ $\%$ lens aperature shadow. This aperture shadow is then divided by uin to generate \% \\ $\%$ the lens array. The lens array is then solved for depth to generate the lens \% \\ $\%$ profile. The remaining functions then plot the input intensity, output intensity, \% \\ $\%$ generated binary lens profile and the generated continuous lens profile. $\%$ \\ $\%$ \\ $\%$ \\ William McCormick, $2004 \%$ \\ \%\%\%\%\%\%\%\%\%\%\%\%\%\%\%\%\%\%\%\%\%\%\%\%\%\%\%\%\%\%\%\%\%\%\%\%\%\%\%\%\%\%\%\%\%\%\%\%\%\%\%\%\%\%\%\%\%\%\%\%\%\%\%\%\%\%\%\%\%\%\%\%

$\begin{array}{ll}\text { clear all } & \text { \%clears workspace } \\ \text { clc } & \text { \%clear command window } \\ \text { home } & \text { \%move cursor to home position in command window } \\ \text { cwd = pwd; } & \text { \%stores location of current work directory in cwd }\end{array}$ \\ pack \\ $\mathrm{cd}(\mathrm{cwd})$ \\ $\% \% \% \% \% \% \% \%$ \\ $\%$ CONSTANTS \% \\ $\% \% \% \% \% \% \% \% \%$

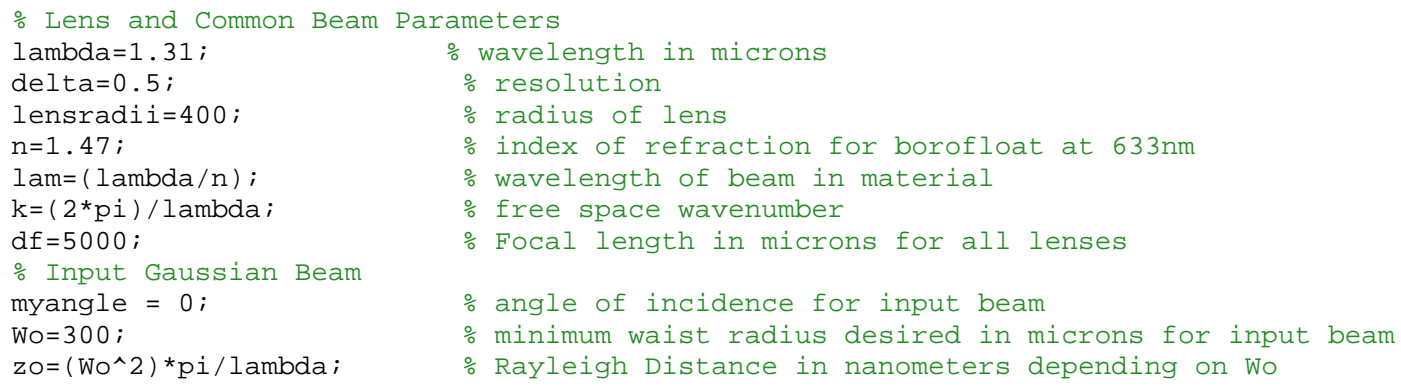


$z=1 e-100$

$\%$ Output Gaussian Beam

Wo2 $=5$;

$\mathrm{zo2}=\left(\mathrm{pi}^{*}(\mathrm{Wo2})^{\wedge} 2\right) / \mathrm{lambda}$;
$\%$ location of waist $\sim \odot$, also location of lens

$\%$ minimum waist desired in microns for output beam or spot

$\%$ Rayleigh Range in meters for output beam

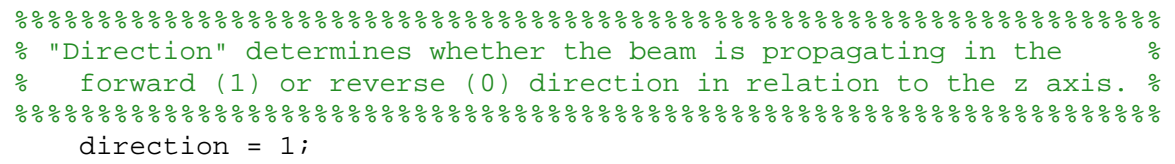

\%\%\%\%\%\%\%\%\%\%\%\%\%\%\%\%\%\%\%\%\%\%\%\%\%\%\%\%\%\%\% 


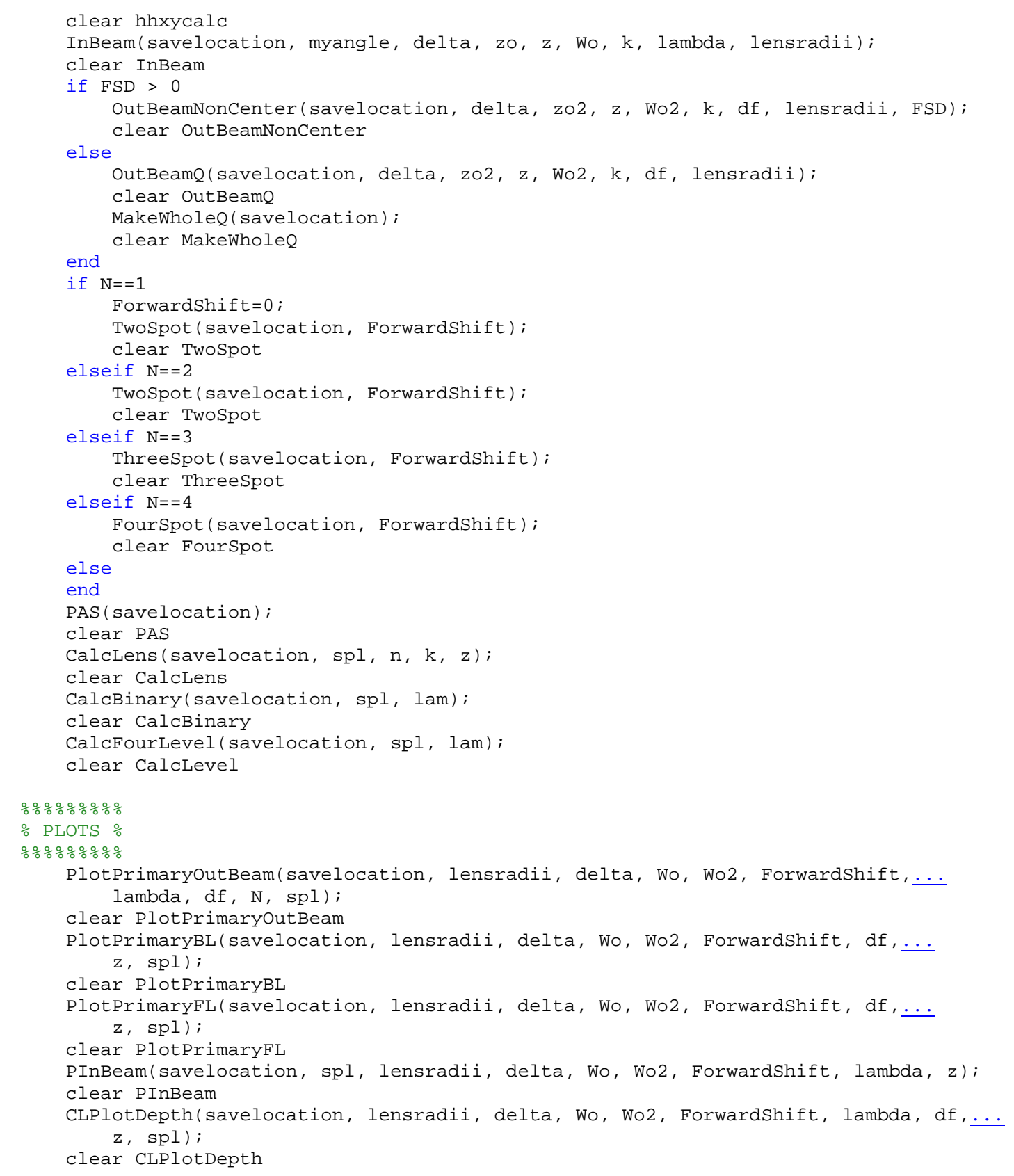




\section{A.2 hhxycalc.m}

function []=hhxycalc(savelocation, lensradii, delta, lambda, k, df)

\%\%\%\%\%\%\%\%\%\%\%\%\%\%\%\%\%\%\%\%\%\%\%\%\%\%\%\%\%\%\%\%\%\%\%\%\%\%\%\%\%\%\%\%\%\%\%\%\%\%\%\%\%\%\%\%\%\%\%\%\%

\% HHXYCALC (savelocation, lensradii, delta, lambda, k, df) \%

\% Calculate the Fresnel Approximation of the Transfer Function of Free space \%

$\%$ using given values. Saves this array as hhxy.mat \%

$\%$ \%

$\% \quad$ William McCormick, $2004 \%$

\%\%\%\%\%\%\%\%\%\%\%\%\%\%\%\%\%\%\%\%\%\%\%\%\%\%\%\%\%\%\%\%\%\%\%\%\%\%\%\%\%\%\%\%\%\%\%\%\%\%\%\%\%\%\%\%\%\%\%\%\%\%\%\%

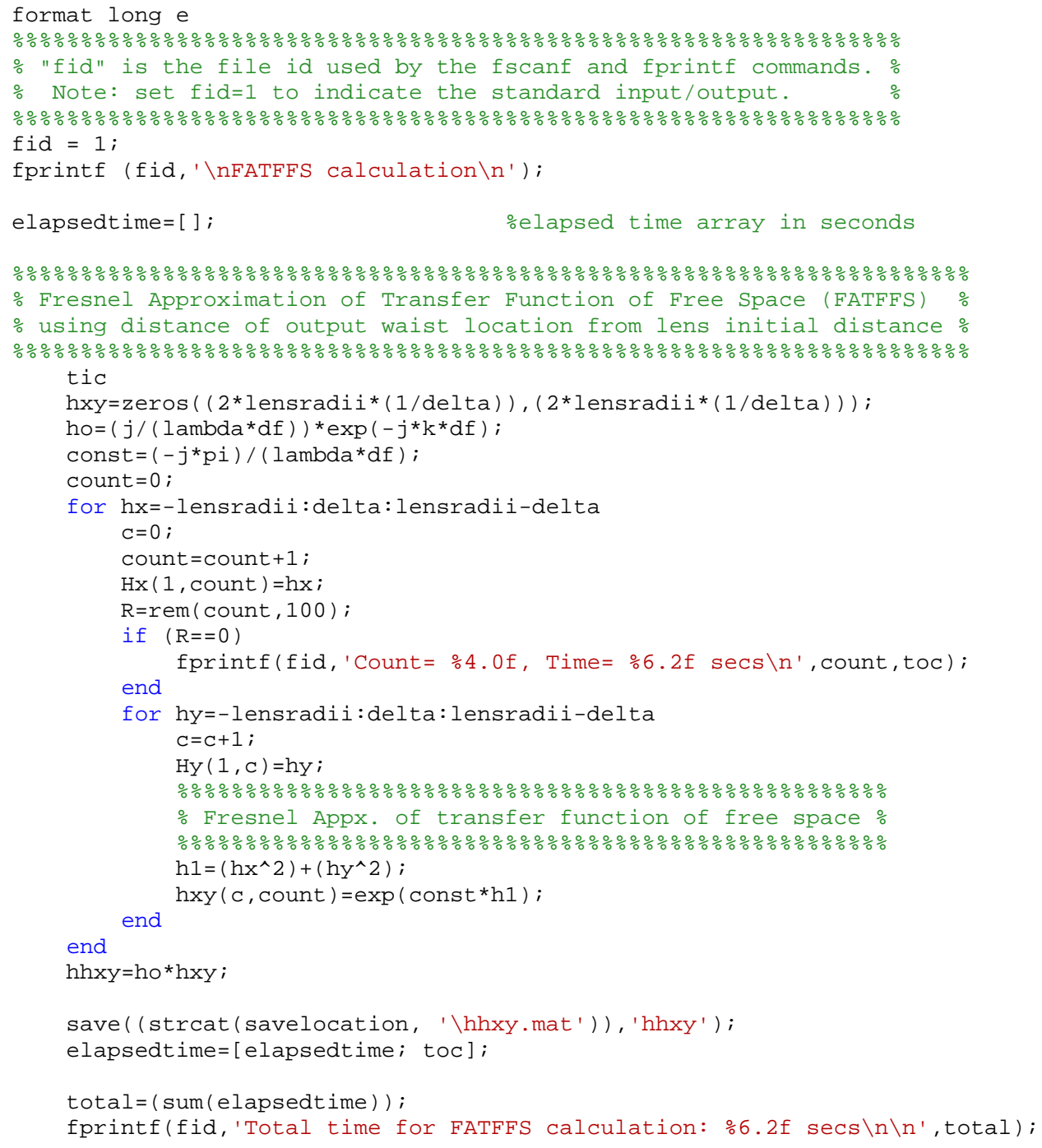




\section{A.3 InBeam.m}

function []=inbeam(savelocation, myangle, delta, zo, z, Wo, k, lambda, lensradii) \%\%\%\%\%\%\%\%\%\%\%\%\%\%\%\%\%\%\%\%\%\%\%\%\%\%\%\%\%\%\%\%\%\%\%\%\%\%\%\%\%\%\%\%\%\%\%\%\%\%\%\%\%\%\%\%\%\%\%\%\%\%\%\%\%\%\%

\% INBEAM(savelocation, myabgle, delta, zo, z, Wo, k, lambda, lensradii)

\% Calculates the 2-D complex Gaussian input beam using parameters for loop

\% size(lensradii) loop resolution(delta), Rayleigh range of input beam(zo

$\%$ of output beam waist in relation to $z$ axis $(z)$, minimum waist radius desired for $\%$

$\%$ input beam(Wo) and the wavenumber(k). Function then saves Gaussian array to file \%

\% PrimaryInput.mat, phase information(bp.mat), posx.mat and posy (posx and posy $\%$

$\%$ allows plot to range from -size to size in x-direction) \%

$\%$
$\%$
$\%$ William McCormick, $2004 \quad \%$

\%\%\%\%\%\%\%\%\%\%\%\%\%\%\%\%\%\%\%\%\%\%\%\%\%\%\%\%\%\%\%\%\%\%\%\%\%\%\%\%\%\%\%\%\%\%\%\%\%\%\%\%\%\%\%\%\%\%\%\%\%\%\%\%\%\%\%\%\%\%\%\%

fid $=1$;

\%\%\%\%\%\%\%\%\%\%\%\%\%\%\%\%\%\%\%\%\%\%\%\%\%\%\%\%\%\%\%\%\%\%\%\%\%\%\%\%\%\%\%\%\%\%\%\%\%\%\%\%

$\%$ "fid" is the file id used by the fscanf and fprintf commands.\%

$\%$ Note: set fid=1 to indicate the standard input/output.

\%\%\%\%\%\%\%\%\%\%\%\%\%\%\%\%\%\%\%\%\%\%\%\%\%\%\%\%\%\%\%\%\%\%\%\%\%\%\%\%\%\%\%\%\%\%\%\%\%\%\%

fprintf (fid, '\nInput beam calculation\n');

\%\%\%\%\%\%\%\%\%\%\%\%\%\%\%\%\%\%\%\%\%\%\%\%\%\%\%\%\%

$\%$ DEFINE VECTORS, MATRICES and ARRAYS \%

\%\%\%\%\%\%\%\%\%\%\%\%\%\%\%\%\%\%\%\%\%\%\%\%\%\%\%\%\%\%

\%convert degrees to radians for calculation

theta=myangle* $\mathrm{pi} / 180$;

\%\%\%\%\%\%\%\%\%\%\%\%\%\%\%\%\%\%\%\%\%\%\%\%\%\%\%\%\%\%\%\%\%\%

$\%$ 2-D COMPLEX GAUSSIAN BEAM AMPLITUDE $U(X) \%$

\%\%\%\%\%\%\%\%\%\%\%\%\%\%\%\%\%\%\%\%\%\%\%\%\%\%\%\%\%\%\%\%\%\%\%\%

tic

elapsedtime $=[] ; \quad$ \%elapsed time array in seconds

\%\%\%\%\%\%\%\%\%\%\%\%\%\%\%\%

$\%$ Initialize arrays \%

$\% \% \% \% \% \% \% \% \% \% \% \% \%$

uin=zeros $((2 *$ lensradii* $(1 /$ delta $)),(2 *$ lensradii* $(1 /$ delta $)))$;

lenuin=length(uin);

for alpha=1: lenuin

for beta=1: lenuin

uin $($ alpha, beta $)=i * 1 e-300$;

end

bp=zeros $((2 *$ lensradii* $(1 /$ delta $)),(2 *$ lensradii* $(1 /$ delta $)))$;

$\operatorname{pos} x=$ zeros $(1,(2 *$ lensradii* $(1 /$ delta $)))$;

$\operatorname{posy}=\operatorname{zeros}(1,(2 *$ lensradii* $(1 /$ delta $)))$;

\%\%\%\%\%\%\%\%\%\%\%\%\%\%\%\%\%\%\%\%\%\%\%\%\%\%\%\%\%\%\%\%\%\%\%\%\%\%\%\%\%\%\%\%\%\%\%\%\%\%\%\%\%\%\%\%\%\%\%\%\%\%\%\%\%\% $\%$ Calculate the bounds for the $x$ and $y$ loops dependent on the angle of incidence \% $\%$ and the location of the plane of interest \%\%\%\%\%\%\%\%\%\%\%\%\%\%\%\%\%\%\%\%\%\%\%\%\%\%\%\%\%\%\%\%\%\%\%\%\%\%\%\%\%\%\%\%\%\%\%\%\%\%\%\%\%\%\%\%\%\%\%\%\%\%\%\%\%\%\% $\%$ LOOK AT PG. 24 OF LAB NOTEBOOK \%

\%\%\%\%\%\%\%\%\%\%\%\%\%\%\%\%\%\%\%\%\%\%\%\%\%\%\%

$\mathrm{x}=\mathrm{z} * \tan ($ theta) ;

bot tom $=x$-lensradii;

top $=x+$ lensradii;

right=lensradii;

left=-lensradii;

$\% \% \% \% \% \% \% \% \% \% \% \%$

\% Calculate Beam \%

\%\%\%\%\%\%\%\%\%\%\%\%\%\%

count $=0$;

for $\mathrm{x}=$ bottom:delta: top-delta

\%\%\%\%\%\%\%\%\%\%\%\%\%\%\%\%\%\%\%\%\%\%\%\%\%\%\%\%\%\%\%\%\%\%\%\%\%\%\%\%\%\%\%\%\%

$\%$ Convert from $x$ and $z$ orientation to xprime and zprime \% 


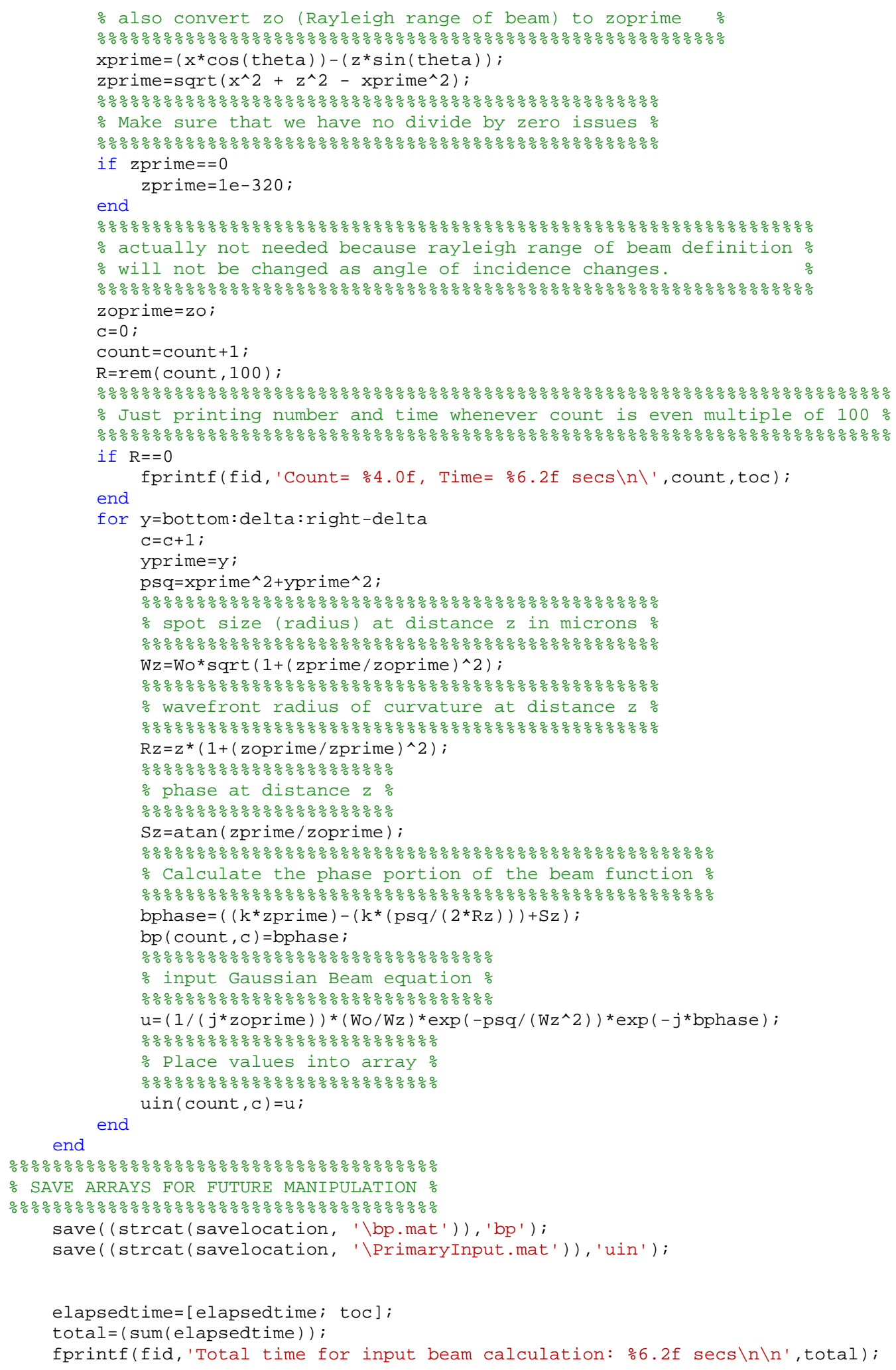




\section{A.4 OutBeamNonCenter.m}

function []=0utBeamNonCenter(savelocation, delta, zo2, z, Wo2, k, df, lensradii, FSD) \%\%\%\%\%\%\%\%\%\%\%\%\%\%\%\%\%\%\%\%\%\%\%\%\%\%\%\%\%\%\%\%\%\%\%\%\%\%\%\%\%\%\%\%\%\%\%\%\%\%\%\%\%\%\%\%\%\%\% \% OUTBEAMNONCENTER(savelocation, delta, zo2, z, Wo2, k, df, lensradii, FSD) \% \% Calculate off-axis 2-D complex Gaussian output beam using parameters for \% $\%$ lens radius(lensradii), loop resolution(delta), Rayleigh range of output \% $\%$ beam(zo2), location of input beam waist in relation to $z$ axis(z), $\%$ minimum waist radius desired for output beam(Wo2) and wavenumber(k). \% \% Function then saves Gaussian array to file Primaryoutput.mat. \% \%\%\%\%\%\%\%\%\%\%\%\%\%\%\%\%\%\%\%\%\%\%\%\%\%\%\%\%\%\%\%\%\%\%\%\%\%\%\%\%\%\%\%\%\%\%\%\%\%\%\%\%\%\%\%\%\%\%\%\%\%\%

fid $=1$; \%\%\%\%\%\%\%\%\%\%\%\%\%\%\%\%\%\%\%\%\%\%\%\%\%\%\%\%\%\%\%\%\%\%\%\%\%\%\%\%\%\%\%\%\%\%\%\%\% $\%$ "fid" is the file id used by the fscanf and fprintf commands. \% $\%$ Note: set fid=1 to indicate the standard input/output. \%\%\%\%\%\%\%\%\%\%\%\%\%\%\%\%\%\%\%\%\%\%\%\%\%\%\%\%\%\%\%\%\%\%\%\%\%\%\%\%\%\%\%\%\%\%\%\%\%\% fprintf (fid, 'Calculation of desired OFF-AXIS output beam after PRIMARY lens $\left.\backslash n^{\prime}\right)$;

elapsedtime=[]; \%elapsed time array in seconds

tic \%\%\%\%\%\%\%\%\%\%\%\%\%\%\%\%\%\%\%\%\%\%\%\%\%\%\%\%\%\%\%

\% Calculated constants for output beam. \% \%\%\%\%\%\%\%\%\%\%\%\%\%\%\%\%\%\%\%\%\%\%\%\%\%\%\%\%\%\%\%\%\%\%\%\%\%\%\%\%\%\%\%\%\%\%\%\%\%\%\%\%\%\%\%\%\%\%\%\%\% $\%$ spot size at distance $z$ in microns, need to use Wo2 because that is the spot \% $\%$ size at the focal point. The focal length of the lens satisfies the $\%$ requirement of the radius of the input beam equal to the radius of the $\%$ output beam. $\mathrm{WZ2}=\mathrm{W} 02 ;$

$\% \% \% \% \% \% \% \% \% \% \% \% \% \% \% \% \% \% \% \% \% \% \% \% \% \% \% \% \% \%$

$\%$ wavefront radius of curvature at distance $z$. \% \%\%\%\%\%\%\%\%\%\%\%\%\%\%\%\%\%\%\%\%\%\%\%\%\%\%\%\%\%\%\%\%\%\%\%\%\%\%\%

$\mathrm{Rz2}=\mathrm{z}^{*}\left(1+(\mathrm{zo} / \mathrm{df})^{\wedge} 2\right)$;

$\% \% \% \% \% \% \% \% \% \% \% \% \% \% \%$

$\%$ phase at distance $z \%$ \%\%\%\%\%\%\%\%\%\%\%\%\%\%\%\%\%\%

$\mathrm{Sz2}=\operatorname{atan}(\mathrm{df} / \mathrm{zo})$;

\%\%\%\%\%\%\%\%\%\%\%\%\%\%\%\%\%\%\%\%\%\%\%\%\%\%\%\%\%\%\%\%\%\%\%\%\%\%\%\%\%\%\%\%\%\%\%\%\%\%

\% Combine some variables that do not need to be recalculated \% \%\%\%\%\%\%\%\%\%\%\%\%\%\%\%\%\%\%\%\%\%\%\%\%\%\%\%\%\%\%\%\%\%\%\%\%\%\%\%\%\%\%\%\%\%\%\%\% $\mathrm{p} 1=(1 /(j * \mathrm{zo} 2)) *($ Wo2/Wz2 $)$;

$\mathrm{p} 2=\left(-j^{*} k^{*} z\right)+\left(j^{*} \mathrm{~S} z 2\right)$;

$\mathrm{p} 3=(j * k) /\left(2{ }^{*} \mathrm{Rz} 2\right)$;

Wz2squared $=W z 2^{\wedge} 2$;

count $=0$;

bottom=lensradii - FSD;

top=lensradii+FSD-delta;

$\% \% \% \% \% \% \% \% \% \%$

$\%$ Define matrix \%

\%\%\%\%\%\%\%\%\%\%\%\%\%

uout $=\operatorname{zeros}\left((2 *\right.$ lensradii* $(1 / \operatorname{delta})),\left(2{ }^{*} \operatorname{lensradii}(1 /\right.$ delta $\left.\left.)\right)\right)$;

for $x=-$ lensradii:delta:lensradii-delta

$\mathrm{c}=0$;

count $=$ count +1 ;

$\mathrm{R}=\mathrm{rem}$ ( count, 100 );

if $(R==0)$

fprintf(fid, 'Count $=\% 4 . \odot f, x=\% 6 . \odot f$, Time $=\% 6.2 f$ secs $\backslash n$ ', count, $x$, toc $)$;

end

for $y=-$ bottom:delta: top

$\mathrm{psq}=\mathrm{x}^{\wedge} 2+\mathrm{y}^{\wedge} 2$;

$\mathrm{c}=\mathrm{c}+1$;

\%\%\%\%\%\%\%\%\%\%\%\%\%\%\%\%\%\%\%\%\%\%\%\%

$\%$ output Gaussian Beam equation \%

$\% \% \% \% \% \% \% \% \% \% \% \% \% \% \% \% \% \% \% \% \% \%$

end

uout $(c$, count $)=\left(p 1^{*} \exp (-p s q / W z 2 s q u a r e d) * \exp \left(p 2-\left(p 3^{*} p s q\right)\right)\right)$; 
end

$\% \% \% \% \% \% \% \% \% \% \% \%$

$\%$ save file name \%

$\% \% \% \% \% \% \% \% \% \% \%$

save(( strcat (savelocation, '\Primaryoutput.mat')), 'uout');

shiftname=strcat ('\PrimaryOutput_shifted_', num2str(FSD, '\%3.0f'), '_um_delta_',... num2str(delta, '\%1.2f'), '_um.mat' );

save((strcat (savelocation, shiftname)), 'uout');

clear uout

elapsedtime=[elapsedtime; toc];

total=( sum(elapsedtime) $)$;

fprintf(fid, 'Total time for output beam calculation: \%6.2f secs $\backslash n \backslash n$ ', total); 


\section{A.5 OutBeamQ.m}

function []=outbeamQ(savelocation, delta, zo2, z, Wo2, k, df, lensradii) \%\%\%\%\%\%\%\%\%\%\%\%\%\%\%\%\%\%\%\%\%\%\%\%\%\%\%\%\%\%\%\%\%\%\%\%\%\%\%\%\%\%\%\%\%\%\%\%\%\%\%\%\%\%\%\% \% OUTBEAMQ (savelocation, delta, zo2, z, Wo2, k, df, lensradii) \%

\% Calculate $1 / 4$ of the on-axis 2-D complex Gaussian output beam using \% $\%$ parameters for lens raduis(lensradii), loop resolution(delta), \%

$\%$ Rayleigh range of output beam(zo2), location of input beam waist in \% $\%$ relation to $z$ axis $(z)$, minimum waist radius desired for output beam \% $\%$ (Wo2) and wavenumber(k). Function then saves Gaussian array to file \% $\%$ Primaryoutput.mat.

format long e

fid $=1$; \%\%\%\%\%\%\%\%\%\%\%\%\%\%\%\%\%\%\%\%\%\%\%\%\%\%\%\%\%\%\%\%\%\%\%\%\%\%\%\%\%\%\%\%\%\%\%\%\%\%\% $\%$ "fid" is the file id used by the fscanf and fprintf commands. \% $\%$ Note: set fid=1 to indicate the standard input/output. \%\%\%\%\%\%\%\%\%\%\%\%\%\%\%\%\%\%\%\%\%\%\%\%\%\%\%\%\%\%\%\%\%\%\%\%\%\%\%\%\%\%\%\%\%\%\%\%\%\%

fprintf (fid, 'Calculation of desired ON-AXIS output beam after PRIMARY lens $\backslash n$ ');

elapsedtime $=[]$;

\%elapsed time array in seconds

tic

\%\%\%\%\%\%\%\%\%\%\%\%\%\%\%\%\%\%\%\%\%\%\%\%\%\%\%\%\%\%\%

\% Calculated constants for output beam. \%

\%\%\%\%\%\%\%\%\%\%\%\%\%\%\%\%\%\%\%\%\%\%\%\%\%\%\%\%\%\%\%\%\%\%\%\%\%\%\%\%\%\%\%\%\%\%\%\%\%\%\%\%\%\%\%\%\%\%\%\%

$\%$ spot size at distance $z$ in microns, need to use Wo2 because that is the spot \%

$\%$ size at the focal point. The focal length of the lens satisfies the

$\%$ requirement of the radius of the input beam equal to the radius of the

$\%$ output beam.

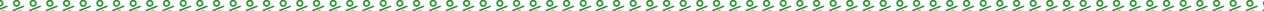
$\mathrm{Wz2}=\mathrm{W} 02$;

\%\%\%\%\%\%\%\%\%\%\%\%\%\%\%\%\%\%\%\%\%\%\%\%\%\%\%\%\%\%\%\%\%\%

$\%$ wavefront radius of curvature at distance $z$. \%

\%\%\%\%\%\%\%\%\%\%\%\%\%\%\%\%\%\%\%\%\%\%\%\%\%\%\%\%\%\%\%\%\%\%\%\%\%\%

$\mathrm{Rz} 2=\mathrm{z}^{*}\left(1+(\mathrm{zo} 2 / \mathrm{df})^{\wedge} 2\right)$;

$\% \% \% \% \% \% \% \% \% \% \% \% \% \% \%$

$\%$ phase at distance z \%

\%\%\%\%\%\%\%\%\%\%\%\%\%\%\%\%\%\%

$\mathrm{Sz2}=\operatorname{atan}(\mathrm{df} / \mathrm{zo} 2)$

\%\%\%\%\%\%\%\%\%\%\%\%\%\%\%\%\%\%\%\%\%\%\%\%\%\%\%\%\%\%\%\%\%\%\%\%\%\%\%\%\%\%\%\%\%\%\%\%\%\%

\% Combine some variables that do not need to be recalculated \% \%\%\%\%\%\%\%\%\%\%\%\%\%\%\%\%\%\%\%\%\%\%\%\%\%\%\%\%\%\%\%\%\%\%\%\%\%\%\%\%\%\%\%\%\%\%\%\%\%

$\mathrm{p} 1=(1 /(j * \mathrm{zo} 2)) *($ Wo2 $/ \mathrm{Wz} 2)$;

$\mathrm{p} 2=\left(-\mathrm{j}^{*} \mathrm{k}^{*} \mathrm{z}\right)+\left(\mathrm{j}^{*} \mathrm{Sz} 2\right)$;

$\mathrm{p} 3=(j * \mathrm{k}) /(2 * \mathrm{Rz} 2)$;

Wz2squared $=\mathrm{Wz2}{ }^{\wedge} 2$;

count $=0$;

$\% \% \% \% \% \% \% \% \% \% \%$

\% Define matrix \%

$\% \% \% \% \% \% \% \% \% \% \%$

uout=zeros ( (lensradii* $(1 /$ delta $)),($ lensradii* $(1 /$ delta $)))$;

for $x=1$ : delta:lensradii-delta

$\mathrm{c}=0$;

count $=$ count +1 ;

$\mathrm{R}=\mathrm{rem}$ ( count, 100$)$;

if $(R==0)$ end

fprintf(fid, 'Count $=\% 4.0 f$, Time $=\% 6.2 f$ secs $\backslash n$ ', count, toc $)$;

for $y=1$ :delta:lensradii-delta

$\mathrm{psq}=\mathrm{x}^{\wedge} 2+\mathrm{y}^{\wedge} 2$;

$\mathrm{C}=\mathrm{C}+1$;

\%\%\%\%\%\%\%\%\%\%\%\%\%\%\%\%\%\%\%\%\%\%\%\%\%

$\%$ output Gaussian Beam equation \%

\%\%\%\%\%\%\%\%\%\%\%\%\%\%\%\%\%\%\%\%\%\%\%\%\%

end

uout $(c$, count $)=p 1^{*} \exp (-p s q /$ Wz2squared $){ }^{*} \exp \left(p 2-\left(p 3^{*} p s q\right)\right)$;

end 
\%\%\%\%\%\%\%\%\%\%\%\%\%\%\%\%

$\%$ save file name \%

\%\%\%\%\%\%\%\%\%\%\%\%\%\%

save((strcat (savelocation, '\Primaryoutput.mat')), 'uout');

clear uout

elapsedtime=[elapsedtime; toc $]$;

total=( sum(elapsedtime) $)$

fprintf(fid, 'Total time for output beam calculation: \%6.2f secs $\backslash n \backslash n$ ', total); 


\section{A.6 MakeWholeQ.m}

function MakewholeQ(savelocation)

\%\%\%\%\%\%\%\%\%\%\%\%\%\%\%\%\%\%\%\%\%\%\%\%\%\%\%\%\%\%\%\%\%\%\%\%\%\%\%\%\%\%\%\%\%\%\%\%\%\%\%\%\%\% $\%$ This function will take the 1/4 field generated by OutBeamQ.m and \% $\%$ manipulate the matrices to form the whole field distribution. \%

$\%$ William McCormick, $2004 \%$

\%\%\%\%\%\%\%\%\%\%\%\%\%\%\%\%\%\%\%\%\%\%\%\%\%\%\%\%\%\%\%\%\%\%\%\%\%\%\%\%\%\%\%\%\%\%\%\%\%\%\%\%\%\%\%\%\%\%

fid $=1$;

\%\%\%\%\%\%\%\%\%\%\%\%\%\%\%\%\%\%\%\%\%\%\%\%\%\%\%\%\%\%\%\%\%\%\%\%\%\%\%\%\%\%\%\%\%\%\%\%\%\%

$\%$ "fid" is the file id used by the fscanf and fprintf commands.\%

$\%$ Note: set fid=1 to indicate the standard input/output.

\%\%\%\%\%\%\%\%\%\%\%\%\%\%\%\%\%\%\%\%\%\%\%\%\%\%\%\%\%\%\%\%\%\%\%\%\%\%\%\%\%\%\%\%\%\%\%\%\%\%\%\%\%

fprintf (fid, '\nConvert 1/4 field to total output field \n');

$\% \% \% \% \% \% \% \% \% \%$

$\%$ Output Beam \%

$\% \% \% \% \% \% \% \% \% \%$

$\operatorname{load}(($ strcat (savelocation, '\Primaryoutput')));

$\% \% \% \% \% \% \% \% \% \% \% \% \% \% \% \% \% \% \% \%$

$\%$ flip matrix left to right \%

$\% \% \% \% \% \% \% \% \% \% \% \% \% \% \% \% \% \% \% \%$

$\mathrm{A}=$ fliplr(uout);

$\% \% \% \% \% \% \% \% \% \% \% \% \% \% \% \% \% \% \% \% \%$

$\%$ combine left and right halves \%

\%\%\%\%\%\%\%\%\%\%\%\%\%\%\%\%\%\%\%\%\%\%\%\%\%\%\%\%\%

$\mathrm{B}=[\mathrm{A}$ uout $]$;

clear A

\%\%\%\%\%\%\%\%\%\%\%\%\%\%\%\%

$\%$ rotate 90 degrees $\%$

$\% \% \% \% \% \% \% \% \% \% \% \% \% \%$

$\mathrm{C}=\operatorname{rot} 90(\mathrm{~B})$;

clear B

$A=f \operatorname{lipl}(C)$;

uout $=\left[\begin{array}{ll}A & C\end{array}\right]$;

clear A

clear C

save((strcat (savelocation, '\Primaryoutput.mat')), 'uout');

clear uout 


\section{A.7 TwoSpot.m}

function twospot(savelocation, ForwardShift)

\%\%\%\%\%\%\%\%\%\%\%\%\%\%\%\%\%\%\%\%\%\%\%\%\%\%\%\%\%\%\%\%\%\%\%\%\%\%\%\%\%\%\%\%\%\%\%\%\%\%\%

\% TWOSPOT(savelocation, ForwardShift, RevShiftout, direction) \%

$\%$ This function will use the matrix Primaryoutput.mat to create a \%

$\%$ new matrix that has two simulated focal points in the output \%

$\%$ plane.

William McCormick, $2004 \%$

\%\%\%\%\%\%\%\%\%\%\%\%\%\%\%\%\%\%\%\%\%\%\%\%\%\%\%\%\%\%\%\%\%\%\%\%\%\%\%\%\%\%\%\%\%\%\%\%\%\%\%\%\%\%\%\%\%\%\%

fid $=1 ; \quad$ \%"fid" is the file id used by the fscanf and fprintf commands \% Note: set fid=1 to indicate the standard input/output

fprintf (fid,' \nshift input and output fields to generate multiple spots $\backslash n '$ );

\%\%\%\%\%\%\%\%\%\%\%\%\%\%\%\%\%\%\%

$\%$ New way to rearrange \%

$\% \% \% \% \% \% \% \% \% \% \% \% \% \% \%$

load((strcat(savelocation, '\Primaryoutput')));

L=length (uout);

fprintf (fid, '\nForward Propagating \n');

$\% \% \% \% \% \% \% \% \% \% \%$

$\%$ put into temps \%

\%\%\%\%\%\%\%\%\%\%\%\%\%\%\%\%\%\%\%\%\%\%\%\%\%\%\%\%\%\%\%\%\%\%\%\%\%\%\%\%\%\%\%\%\%\%\%\%

$\%$ put the beginning of the matrix into a temp location $\%$ \%\%\%\%\%\%\%\%\%\%\%\%\%\%\%\%\%\%\%\%\%\%\%\%\%\%\%\%\%\%\%\%\%\%\%\%\%\%\%\%\%\%\%\%

$\mathrm{tL}=$ uout $(1: \mathrm{L}, 1$ : ForwardShift ) ;

\%\%\%\%\%\%\%\%\%\%\%\%\%\%\%\%\%\%\%\%\%\%\%\%\%\%\%\%\%\%\%\%\%\%\%

$\%$ put the end of the matrix in a temp location \%

\%\%\%\%\%\%\%\%\%\%\%\%\%\%\%\%\%\%\%\%\%\%\%\%\%\%\%\%\%\%\%\%\%\%\%\%\%\%\%

tR=uout $(1: L, 1: L-$ ForwardShift $)$;

\%\%\%\%\%\%\%\%\%\%\%\%

$\%$ shift array \%

\%\%\%\%\%\%\%\%\%\%\%\%\%\%\%\%\%\%\%\%\%\%\%\%

$\%$ shift matrix to the left \%

$\% \% \% \% \% \% \% \% \% \% \% \% \% \% \% \% \% \%$

$\mathrm{TL}=\operatorname{uout}(1: \mathrm{L}$, ForwardShift $+1: \mathrm{L})$;

\%\%\%\%\%\%\%\%\%\%\%\%\%\%\%\%\%\%\%\%\%\%\%\%\%

$\%$ shift the matrix to the right \%

$\% \% \% \% \% \% \% \% \% \% \% \% \% \% \% \% \% \% \% \% \%$

$\operatorname{TR}=\operatorname{uout}(1: \mathrm{L},(\mathrm{L}-$ ForwardShift $)+1: \mathrm{L})$

\%\%\%\%\%\%\%\%\%\%\%\%\%\%\%\%\%\%\%\%\%\%

$\%$ put array back together \%

\%\%\%\%\%\%\%\%\%\%\%\%\%\%\%\%\%\%\%\%

uoutshiftleft $=[\mathrm{TL} \mathrm{tL}]$;

uoutshiftright $=\left[\begin{array}{ll}T R & t R\end{array}\right]$;

\%\%\%\%\%\%\%\%\%\%\%\%\%\%\%\%\%\%

$\%$ Add beams together \%

$\% \% \% \% \% \% \% \% \% \% \% \% \% \%$

uout=uoutshiftleft+uoutshiftright;

save((strcat(savelocation, '\Primaryoutput.mat')), 'uout'); 


\section{A.8 ThreeSpot.m}

function threespot(savelocation, ForwardShift)

\%\%\%\%\%\%\%\%\%\%\%\%\%\%\%\%\%\%\%\%\%\%\%\%\%\%\%\%\%\%\%\%\%\%\%\%\%\%\%\%\%\%\%\%\%\%\%\%\%\%\%\%

$\%$ THREESPOT( savelocation, ForwardShift)

$\%$ This function will use the matrix Primaryoutput.mat to create a \%

$\%$ new matrix that has three simulated focal points in the output \%

$\%$ plane.

William McCormick, 2004

\%\%\%\%\%\%\%\%\%\%\%\%\%\%\%\%\%\%\%\%\%\%\%\%\%\%\%\%\%\%\%\%\%\%\%\%\%\%\%\%\%\%\%\%\%\%\%\%\%\%\%\%\%\%\%

fid $=1 ; \quad$ \%"fid" is the file id used by the fscanf and fprintf commands

\% Note: set fid=1 to indicate the standard input/output

fprintf (fid,' \nshift input and output fields to generate multiple spots $\backslash n \backslash n ')$;

\%\%\%\%\%\%\%\%\%\%\%\%\%\%\%\%\%\%\%

$\%$ New way to rearrange \%

$\% \% \% \% \% \% \% \% \% \% \% \% \% \% \%$

$\operatorname{load}(($ strcat(savelocation, '\Primaryoutput')));

uoutshiftleft=uout;

uoutshiftright=uout;

L=length(uout);

clear uout

$\% \% \% \% \% \% \% \% \% \% \% \% \%$

$\%$ put into temps $\%$

\%\%\%\%\%\%\%\%\%\%\%\%\%\%\%\%\%\%\%\%\%\%\%\%\%\%\%\%\%\%\%\%\%\%\%\%\%\%\%\%\%\%\%\%\%\%

$\%$ put the beginning of the matrix into a temp location \%

\%\%\%\%\%\%\%\%\%\%\%\%\%\%\%\%\%\%\%\%\%\%\%\%\%\%\%\%\%\%\%\%\%\%\%\%\%\%\%\%\%\%

$\mathrm{tL}=$ uoutshiftleft (1: L, 1: ForwardShift) ;

\%\%\%\%\%\%\%\%\%\%\%\%\%\%\%\%\%\%\%\%\%\%\%\%\%\%\%\%\%\%\%\%\%\%\%\%\%

$\%$ put the end of the matrix in a temp location \%

\%\%\%\%\%\%\%\%\%\%\%\%\%\%\%\%\%\%\%\%\%\%\%\%\%\%\%\%\%\%\%\%\%\%\%\%\%\%

tR=uoutshiftright (1:L, 1:L-ForwardShift)；

$\% \% \% \% \% \% \% \% \% \%$

$\%$ shift array \%

\%\%\%\%\%\%\%\%\%\%\%\%\%\%\%\%\%\%\%\%\%\%\%\%

$\%$ shift matrix to the left $\%$

\%\%\%\%\%\%\%\%\%\%\%\%\%\%\%\%\%\%\%\%\%

$\mathrm{TL}=$ uoutshiftleft $(1: L$, ForwardShift $+1: L)$;

\%\%\%\%\%\%\%\%\%\%\%\%\%\%\%\%\%\%\%\%\%\%\%\%\%

$\%$ shift the matrix to the right \%

\%\%\%\%\%\%\%\%\%\%\%\%\%\%\%\%\%\%\%\%\%\%

TR=uoutshiftright (1:L, (L-ForwardShift) $+1: L)$;

\%\%\%\%\%\%\%\%\%\%\%\%\%\%\%\%\%\%\%\%\%

$\%$ put array back together \%

\%\%\%\%\%\%\%\%\%\%\%\%\%\%\%\%\%\%\%\%

uoutshiftleft $=[\mathrm{TL} \mathrm{tL}]$;

uoutshiftright $=[$ TR $\mathrm{tR}]$;

$\% \% \% \% \% \% \% \% \% \% \% \% \% \%$

$\%$ Add beams together \%

$\% \% \% \% \% \% \% \% \% \% \% \% \% \%$

uout=uoutshiftleft+uoutshiftright+uout ;

save((strcat (savelocation, '\Primaryoutput.mat')), 'uout'); 


\section{A.9 FourSpot.m}

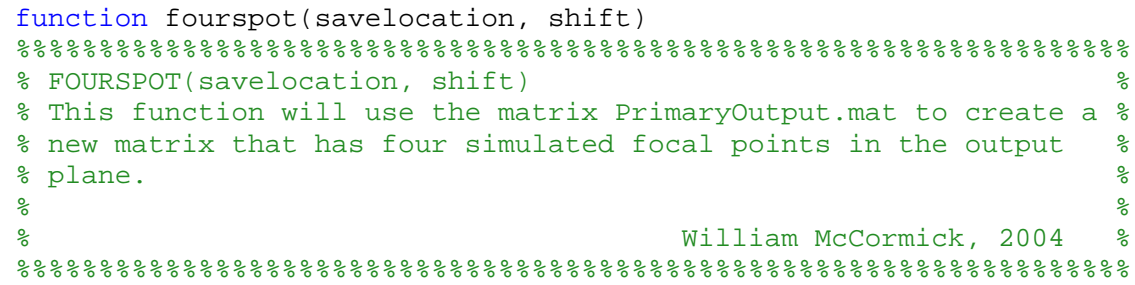

\%\%\%\%\%\%\%\%\%\%\%\%

$\%$ shift array \%

\%\%\%\%\%\%\%\%\%\%\%\%\%\%\%\%\%\%\%\%\%\%\%\%\%\%\%

$\%$ shift matrix to the left $\%$

\%\%\%\%\%\%\%\%\%\%\%\%\%\%\%\%\%\%\%\%\%\%\%

TL=uoutshiftleft $(1: \mathrm{L}$, shift+1: L ) ;

\%\%\%\%\%\%\%\%\%\%\%\%\%\%\%\%\%\%\%\%\%\%\%\%\%

$\%$ shift the matrix to the right \%

\%\%\%\%\%\%\%\%\%\%\%\%\%\%\%\%\%\%\%\%\%\%\%\%\%

TR=uoutshiftright $(1: L,(L-s h i f t)+1: L)$;

\%\%\%\%\%\%\%\%\%\%\%\%\%\%\%\%\%\%\%\%\%\%\%

$\%$ put arrays back together $\%$

\%\%\%\%\%\%\%\%\%\%\%\%\%\%\%\%\%\%\%\%\%\%

uoutshiftleft $=[T L \mathrm{tL}]$;

uoutshiftright $=[\mathrm{TR}$ tR $]$;

\%\%\%\%\%\%\%\%\%\%\%\%\%\%\%\%\%\%

$\%$ Add beams together $\%$

\%\%\%\%\%\%\%\%\%\%\%\%\%\%\%\%\%

uoutsubtotal=ur2shiftleft+ur2shiftright ;

A=uoutsubtotal';

uoutshiftleft=A;

uoutshiftright=A;

$\mathrm{L}=$ length $(\mathrm{A})$;

\%\%\%\%\%\%\%\%\%\%\%\%\%\%\%

$\%$ put into temps $\%$

\%\%\%\%\%\%\%\%\%\%\%\%\%\%\%\%\%\%\%\%\%\%\%\%\%\%\%\%\%\%\%\%\%\%\%\%\%\%\%\%\%\%\%\%\%\%\%\%\%

$\%$ put the beginning of the matrix into a temp location $\%$ \%\%\%\%\%\%\%\%\%\%\%\%\%\%\%\%\%\%\%\%\%\%\%\%\%\%\%\%\%\%\%\%\%\%\%\%\%\%\%\%\%\%\%\%\%\%\%

t $\mathrm{L}=$ uoutshiftleft $(1: \mathrm{L}, 1$ : shift ) ;

\%\%\%\%\%\%\%\%\%\%\%\%\%\%\%\%\%\%\%\%\%\%\%\%\%\%\%\%\%\%\%\%\%\%\%\%\%\%\%

$\%$ put the end of the matrix in a temp location \%

\%\%\%\%\%\%\%\%\%\%\%\%\%\%\%\%\%\%\%\%\%\%\%\%\%\%\%\%\%\%\%\%\%\%\%\%\%\%\%\%\% 
tR=uoutshiftright $(1: L, 1: L$-shift $)$;

\%\%\%\%\%\%\%\%\%\%\%\%

$\%$ shift array \%

\%\%\%\%\%\%\%\%\%\%\%\%\%\%\%\%\%\%\%\%\%\%\%\%\%\%

$\%$ shift matrix to the left \%

\%\%\%\%\%\%\%\%\%\%\%\%\%\%\%\%\%\%\%\%\%

$\mathrm{TL}=$ uout shiftleft $(1: \mathrm{L}$, shift $+1: \mathrm{L})$;

\%\%\%\%\%\%\%\%\%\%\%\%\%\%\%\%\%\%\%\%\%\%\%\%\%

$\%$ shift the matrix to the right \%

\%\%\%\%\%\%\%\%\%\%\%\%\%\%\%\%\%\%\%\%\%\%\%\%\%

TR=uoutshiftright (1:L, (L-shift $)+1: L)$;

\%\%\%\%\%\%\%\%\%\%\%\%\%\%\%\%\%\%\%\%\%\%

$\%$ put arrays back together \%

\%\%\%\%\%\%\%\%\%\%\%\%\%\%\%\%\%\%\%\%

uoutshiftleft $=[\mathrm{TL} \mathrm{tL}]$;

uoutshiftright=[TR tR];

uout=uoutshiftleft+uoutshiftright;

save((strcat(savelocation, '\Primaryoutput.mat')), 'uout'); 


\section{A.10 PAS.m}

function []=PAS (savelocation)

\%\%\%\%\%\%\%\%\%\%\%\%\%\%\%\%\%\%\%\%\%\%\%\%\%\%\%\%\%\%\%\%\%\%\%\%\%\%\%\%\%\%\%\%\%\%\%\%\%\%\%\%\%\%\%\%\% \% FAS(savelocation)

\% Calculate the aperture shadow of the lens using the desired

$\%$ output and the impulse response transfer function. Saves this

$\%$ array as PAS.mat (Primary Aperture Shadow).

$\%$

\%\%\%\%\%\%\%\%\%\%\%\%\%\%\%\%\%\%\%\%\%\%\%\%\%\%\%\%\%\%\%\%\%\%\%\%\%\%\%\%\%\%\%\%\%\%\%\%\%\%\%\%\%\%\%\%\%\%

load((strcat(savelocation, '\hhxy')));

load((strcat(savelocation, '\Primaryoutput')));

FAS=fftshift (ifft2((fft2(uout) $). /(f f t 2($ hhxy $))))$;

save( (strcat (savelocation, '\PrimaryApertureshadow.mat')), 'FAS');

clear FAS; 


\section{A.11 CalcLens.m}

function []=CalcLens (savelocation, spl, $\mathrm{n}, \mathrm{k}, \mathrm{z}$ )

\%\%\%\%\%\%\%\%\%\%\%\%\%\%\%\%\%\%\%\%\%\%\%\%\%\%\%\%\%\%\%\%\%\%\%\%\%\%\%\%\%\%\%\%\%\%\%\%\%\%\%

\% CalcLens(savelocation, spl, n, k, z)

\% Calculate the lens profile using the input beam array and the \%

$\%$ aperture shadow array

$\%$

fid $=1 ; \quad$ \%"fid" is the file id used by the fscanf and fprintf commands

$\%$ Note: set $f i d=1$ to indicate the standard input/output

if direction

fprintf (fid, '\nPRIMARY continuous profile calculation $\backslash n$ ');

load((strcat (savelocation, '\PrimaryInput')));

load((strcat (savelocation, '\PrimaryApertureshadow')));

LensArray=FAS . /uin;

filename $1=($ '\LensArray $\cdot$ mat' $)$;

else

filename2 $=($ '\PrimaryDepth.mat' $) ;$

fprintf (fid,' \nSECONDARY continuous profile calculation $\backslash n ')$;

load((strcat (savelocation, '\PrimaryApertureshadow')));

load((strcat (savelocation, '\SecondaryApertureshadow')));

LensAr ray=RAS . /FAS;

filename1 $=($ ' $\backslash$ SecondaryLensArray. mat' $)$;

end

filename2 = ('\SecondaryDepth. mat' $)$;

\%\%\%\%\%\%\%\%\%\%\%\%\%\%\%\%\%\%\%\%\%\%\%\%\%\%\%\%\%\%\%\%\%\%\%\%\%\%\%\%\%\%\%\%\%\%\% \% CALCULATE THE DEPTH OF THE LENS PROFILE ACCORDING TO PHASE \% \% INFORMATION OBTAINED FROM THE LENS ARRAY

\%\%\%\%\%\%\%\%\%\%\%\%\%\%\%\%\%\%\%\%\%\%\%\%\%\%\%\%\%\%\%\%\%\%\%\%\%\%\%\%\%\%\%\%\%\%

depth $=(\log ($ LensArray $)) /(-j * n * k)$;

$\% \% \% \% \% \% \% \% \% \% \% \% \% \% \% \%$

$\%$ find the minimum value \%

\%\%\%\%\%\%\%\%\%\%\%\%\%\%\%\%\%\%\%\%

$\mathrm{md}=\operatorname{abs}(\min (\min (\operatorname{real}(\operatorname{depth}))))$;

\%\%\%\%\%\%\%\%\%\%\%\%\%\%\%\%\%\%\%

$\%$ shift profile to zero \%

$\% \% \% \% \% \% \% \% \% \% \% \% \% \% \% \% \% \%$

depth $=($ depth+md $)$;

save(( $\operatorname{strcat}(\mathrm{spl}$, filename1)), 'LensArray'):

save (( strcat (spl, filename2)), 'depth'); 


\section{A.13 PlotPrimaryOutBeam.m}

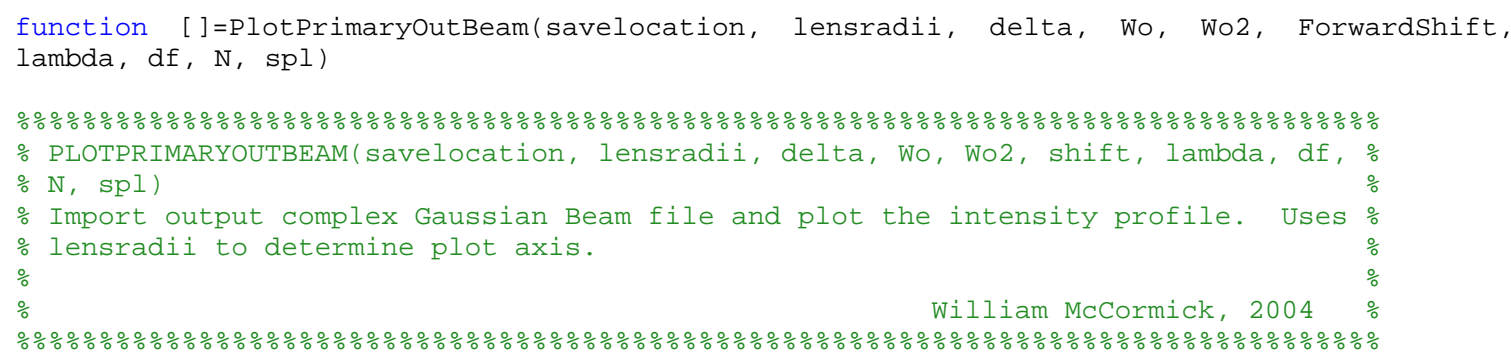

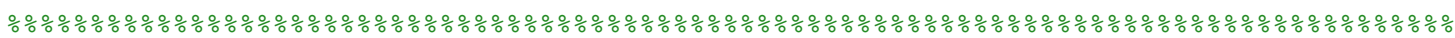
$\%$ sets the fontweight and fontsize for current plot for a 3 inch wide picture in word \% \%\%\%\%\%\%\%\%\%\%\%\%\%\%\%\%\%\%\%\%\%\%\%\%\%\%\%\%\%\%\%\%\%\%\%\%\%\%\%\%\%\%\%\%\%\%\%\%\%\%\%\%\%\%\%\%\%\%\%\%\%\%\%\%\%\%\%\%\%\%\% set (gca, 'fontweight', 'demi', 'fontsize', 18);

\%\%\%\%\%\%\%\%\%\%\%\%\%\%\%\%\%\%\%\%\%\%\%\%\%\%\%\%\%\%\%\%\%\%\%\%\%\%\%\%\%\%\%\%\%\%\%\%\%\%\%\%\%\%\%\%\%\%\%\%\%\%\%\%\%\%\%\%\% $\%$ sets the fontweight and fontsize for current plot for a 5 inch wide picture in word \% 
\%\%\%\%\%\%\%\%\%\%\%\%\%\%\%\%\%\%\%\%\%\%\%\%\%\%\%\%\%\%\%\%\%\%\%\%\%\%\%\%\%\%\%\%\%\%\%\%\%\%\%\%\%\%\%\%\%\%\%\%\%\%\%\%\%\%\%\%\%\%\% $\%$ set(gca, 'fontweight', 'demi', 'fontsize',14);

\%\%\%\%\%\%\%\%\%\%\%\%\%\%\%\%\%\%\%\%\%\%\%\%\%\%\%\%\%\%\%\%\%\%\%\%\%\%\%\%\%\%\%\%\%\%\%\%\%\%\%\%\%\%\%\%\%\%\%\%\%\%\%\%\%\%\% $\%$ sets the fontweight and fontsize for current plot for a 6 inch wide picture in word \% \%\%\%\%\%\%\%\%\%\%\%\%\%\%\%\%\%\%\%\%\%\%\%\%\%\%\%\%\%\%\%\%\%\%\%\%\%\%\%\%\%\%\%\%\%\%\%\%\%\%\%\%\%\%\%\%\%\%\%\%\%\%\%\%\%\%\%\% $\% \quad \operatorname{set}($ gca, 'fontweight', 'demi', 'fontsize',10);

halfshift=ForwardShift/2;

title(\{['Output beam (resolution = ', num2str(delta, '\%1.2f'), ' um, shift= ', num2str (halfshift...

' '\%3.of'), 'um, lambda = ', num2str(lambda, '\%1.3f'), ' um)']...

$;[$ ' (input Wo $=$ ', num2str (Wo, '\%4.Of'), ' um, desired output Wo $\overline{\bar{\prime}}$ ', num2str(Wo2...

xlabel ('X (úm)');

'\%4.of'),' um, focal dis. = ', num2str(df, '\%5.of'),' um)']\})'

ylabel('Y (um)')'

zlabel('Normalized Intensity');

$\% \% \% \% \% \% \% \% \% \% \% \% \% \%$

\% Plot Top view \%

$\% \% \% \% \% \% \% \% \% \% \% \% \%$

$\%$ Sets plot window to close to full size

figure('Position', [scrsz(1)+4 scrsz(2) scrsz(3) scrsz(4)-45], 'color', [1 11 1])

$\%$ mesh(posx, posy, Imax2, 'FaceColor', 'interp', ...

\% 'FaceLighting', 'phong', 'Linestyle', 'none', 'EdgeLighting ', 'phong' );

\%\%\%\%\%\%\%\%\%\%\%\%\%\%\%\%\%\%\%\%\%\%\%\%\%\%\%\%\%\%\%\%\%\%\%\%\%\%\%\%\%\%

$\%$ To print just a portion of the array and save memory \%

\%\%\%\%\%\%\%\%\%\%\%\%\%\%\%\%\%\%\%\%\%\%\%\%\%\%\%\%\%\%\%\%\%\%\%\%\%\%\%\%\%\%\%

mesh(posx(700:901), posy(100:301), Imax2(100:301,700:901), 'FaceColor', 'interp', $\ldots$ 'Facelighting', 'phong', 'LineStyle', 'none', 'EdgeLighting', 'phong' ');

axis( 'square');

view $(0,90)$;

light('Position', [1 10 0], 'Color', [0.498 0.498 0.498], 'Style','infinite');

light ('Position', [ $\left.\begin{array}{lll}-1 & -1 & 0\end{array}\right]$, 'Color', [0.498 0.4980 .498$]$, 'Style', 'infinite');

light('Position', [-1 10 , ',Color', [0.498 0.498 0.498],' 'Style', 'infinite'):

light('Position', [1 -10 ], 'Color', [0.498 0.498 0.498], 'Style', 'infinite');

light ('Position', [ $\left.\begin{array}{lll}0 & \odot & 1\end{array}\right]$, 'Style', 'infinite');

grid off

\%\%\%\%\%\%\%\%\%\%\%\%\%\%\%\%\%\%\%\%\%\%\%\%\%\%\%\%\%\%\%\%\%\%\%\%\%\%\%\%\%\%\%\%\%\%\%\%\%\%\%\%\%\%\%\%\%\%\%\%\%\%\%\%\%\% $\%$ sets the fontweight and fontsize for current plot for a 3 inch wide picture in word \% \%\%\%\%\%\%\%\%\%\%\%\%\%\%\%\%\%\%\%\%\%\%\%\%\%\%\%\%\%\%\%\%\%\%\%\%\%\%\%\%\%\%\%\%\%\%\%\%\%\%\%\%\%\%\%\%\%\%\%\%\%\%\%\%\%\%\%\%\% set (gca, 'fontweight ', 'demi', 'fontsize', 18);

\%\%\%\%\%\%\%\%\%\%\%\%\%\%\%\%\%\%\%\%\%\%\%\%\%\%\%\%\%\%\%\%\%\%\%\%\%\%\%\%\%\%\%\%\%\%\%\%\%\%\%\%\%\%\%\%\%\%\%\%\%\%\%\%\%\%\% $\%$ sets the fontweight and fontsize for current plot for a 5 inch wide picture in word \% \%\%\%\%\%\%\%\%\%\%\%\%\%\%\%\%\%\%\%\%\%\%\%\%\%\%\%\%\%\%\%\%\%\%\%\%\%\%\%\%\%\%\%\%\%\%\%\%\%\%\%\%\%\%\%\%\%\%\%\%\%\%\%\%\%\%\% $\% \quad \operatorname{set}($ gca, 'fontweight ', 'demi', 'fontsize', 14);

\%\%\%\%\%\%\%\%\%\%\%\%\%\%\%\%\%\%\%\%\%\%\%\%\%\%\%\%\%\%\%\%\%\%\%\%\%\%\%\%\%\%\%\%\%\%\%\%\%\%\%\%\%\%\%\%\%\%\%\%\%\%\%\%\%\%\% $\%$ sets the fontweight and fontsize for current plot for a 6 inch wide picture in word \% \%\%\%\%\%\%\%\%\%\%\%\%\%\%\%\%\%\%\%\%\%\%\%\%\%\%\%\%\%\%\%\%\%\%\%\%\%\%\%\%\%\%\%\%\%\%\%\%\%\%\%\%\%\%\%\%\%\%\%\%\%\%\%\%\%\%\% $\% \quad \operatorname{set}(g c a$, 'fontweight', 'demi', 'fontsize',10);

halfshift=ForwardShift/2;

title(\{['Output beam (resolution = ', num2str(delta, $\left.\% 1.2 f^{\prime}\right)$, ' um, shift = ', num2str(halfshift...

,'\%3.of'), 'um, lambda = ', num2str(lambda, '\%1.3f'), ' um)']...

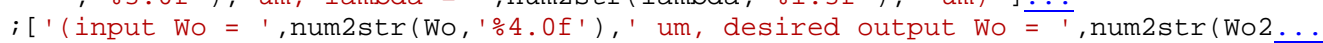

xlabel ('X (um)' ');

'\%4.of'),' um, focal dis. = ', num2str(df, '\%5.of'),' um)']\});

ylabel('Y (um)');

zlabel('Normalized Intensity');

$\% \% \% \% \% \% \% \% \% \% \% \% \% \%$

$\%$ Plot side view \%

\%\%\%\%\%\%\%\%\%\%\%\%\%\%\% 


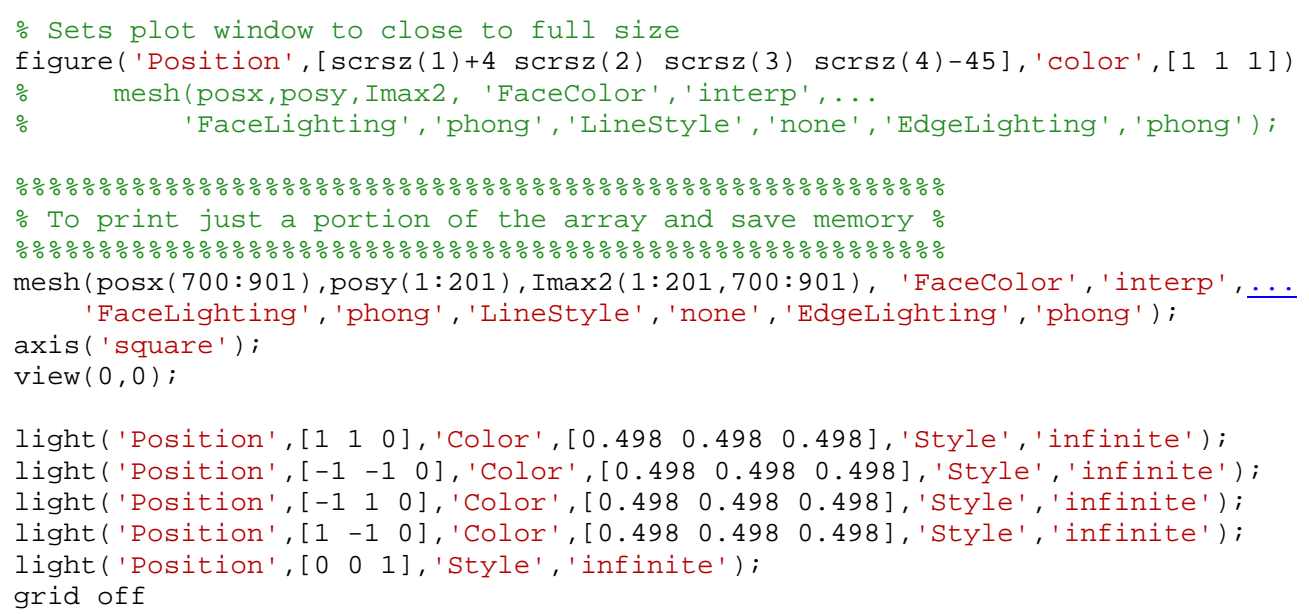

filename=strcat (spl, '\PrimaryoutputWo', num2str(Wo), 'umN', num2str(N), 'S', num2str(ForwardSh 


\section{A.14 PlotPrimaryBL.m}

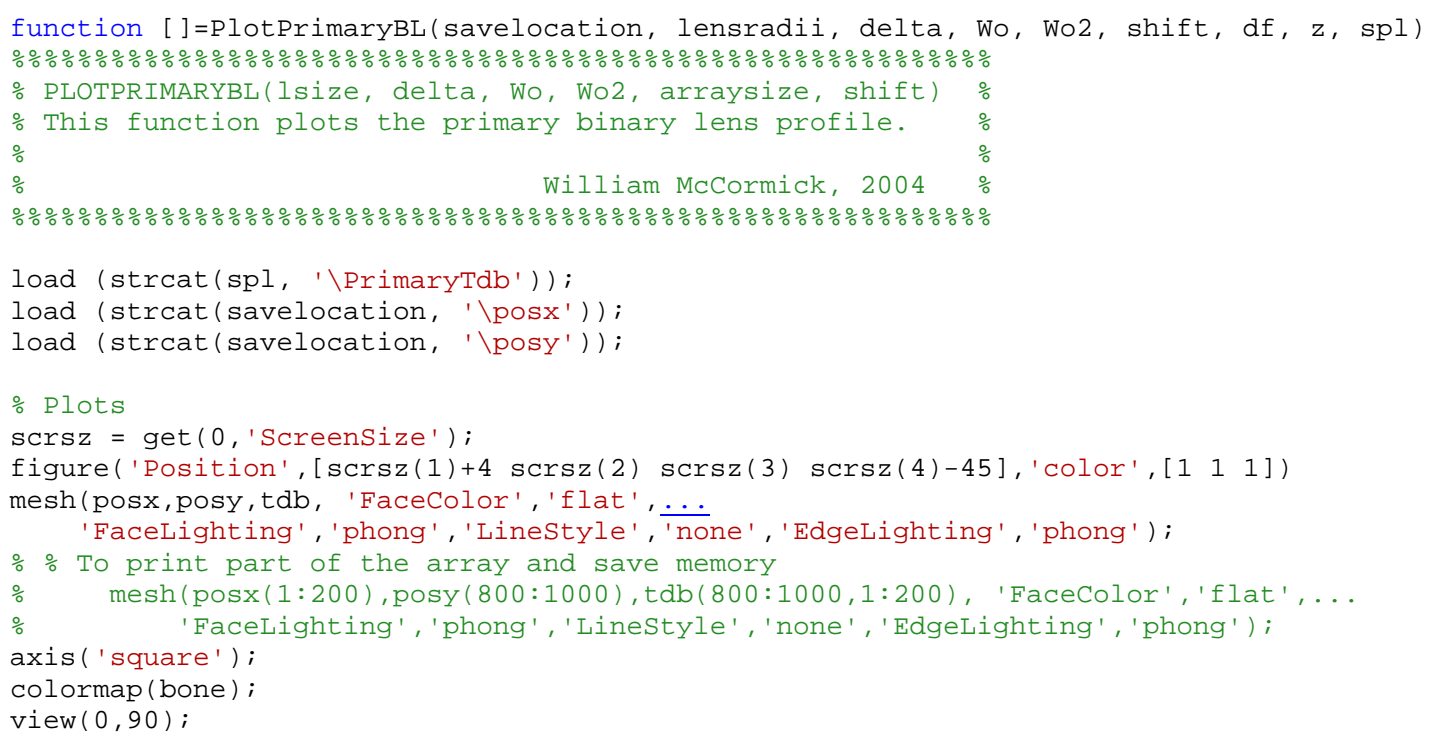

\%\%\%\%\%\%\%\%\%\%\%\%\%\%\%\%\%\%\%\%\%\%\%\%\%\%\%\%\%\%\%\%\%\%\%\%\%\%\%\%\%\%\%\%\%\%\%\%\%\%\%\%\%\%\%\%\%\%\%\%\%\%\%\%\%\%\%\%\%\% $\%$ sets the fontweight and fontsize for current plot for a 3 inch wide picture in word \% \%\%\%\%\%\%\%\%\%\%\%\%\%\%\%\%\%\%\%\%\%\%\%\%\%\%\%\%\%\%\%\%\%\%\%\%\%\%\%\%\%\%\%\%\%\%\%\%\%\%\%\%\%\%\%\%\%\%\%\%\%\%\%\%\%\%\%\%\%\%\% set(gca, 'fontweight ', 'demi', 'fontsize',18);

\%\%\%\%\%\%\%\%\%\%\%\%\%\%\%\%\%\%\%\%\%\%\%\%\%\%\%\%\%\%\%\%\%\%\%\%\%\%\%\%\%\%\%\%\%\%\%\%\%\%\%\%\%\%\%\%\%\%\%\%\%\%\%\%\% $\%$ sets the fontweight and fontsize for current plot for a 5 inch wide picture in word \% \%\%\%\%\%\%\%\%\%\%\%\%\%\%\%\%\%\%\%\%\%\%\%\%\%\%\%\%\%\%\%\%\%\%\%\%\%\%\%\%\%\%\%\%\%\%\%\%\%\%\%\%\%\%\%\%\%\%\%\%\%\%\%\% $\% \quad \operatorname{set}($ gca, ' fontweight', 'demi', 'fontsize', 14);

\%\%\%\%\%\%\%\%\%\%\%\%\%\%\%\%\%\%\%\%\%\%\%\%\%\%\%\%\%\%\%\%\%\%\%\%\%\%\%\%\%\%\%\%\%\%\%\%\%\%\%\%\%\%\%\%\%\%\%\%\%\%\%\%\%\%\% $\%$ sets the fontweight and fontsize for current plot for a 6 inch wide picture in word \% \%\%\%\%\%\%\%\%\%\%\%\%\%\%\%\%\%\%\%\%\%\%\%\%\%\%\%\%\%\%\%\%\%\%\%\%\%\%\%\%\%\%\%\%\%\%\%\%\%\%\%\%\%\%\%\%\%\%\%\%\% $\% \quad \operatorname{set}($ gca, 'fontweight', 'demi', 'fontsize', 10);

title(\{['Primary binary lens profile (delta = ', num2str(delta, '\%1.2f'), ' um, input wo = '...

, num2str(Wo, '\%4.of'), ' um)']; ['(waist loc. = ', num2str(z, '\%4.0f'), ...

' um, output Wo = ', num2str(Wo2, '\%4.Of'),' um, shift =

', num2str(shift/(2), '\%3. of' '), ...

' um, focal dis. = ', $\left.\left.\left.\left.\operatorname{num} 2 \operatorname{str}\left(\mathrm{df}, \mathrm{\prime}^{\circ} \mathrm{5} . \mathrm{of} \mathrm{f}^{\prime}\right),{ }^{\prime} \mathrm{um}\right)^{\prime}\right]\right\}\right)$;

xlabel('X (um)');

ylabel('Y (um)')'

zlabel('Depth of lens profile (nm)');

filename=strcat (spl, '\PrimaryL', num2str(2* lensradii), 'umBWo', num2str(Wo), ...

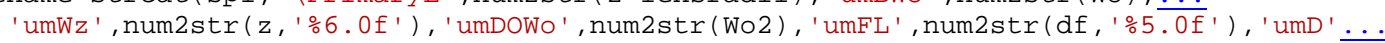
, num2str(delta, '\%1.2f'), 'um.fig');

saveas (gcf, filename); 


\section{A.15 PinBeam.m}

function []=PInBeam(savelocation, spl, lensradii, delta, Wo, Wo2, shift, lambda, z) \%\%\%\%\%\%\%\%\%\%\%\%\%\%\%\%\%\%\%\%\%\%\%\%\%\%\%\%\%\%\%\%\%\%\%\%\%\%\%\%\%\%\%\%\%\%\%\%\%\%\%\%\%\%\%\%\%\%\%\%\%\%\% \% PINBEAMBEAM(savelocation, spl, lensradii, delta, Wo, Wo2, shift, lambda, z) \% \% Import input complex Gaussian Beam file and plot the intensity profile. \% $\%$ Uses lensradii to determine plot axis.

$\%$

$\%$

William McCormick, $2004 \%$

\%\%\%\%\%\%\%\%\%\%\%\%\%\%\%\%\%\%\%\%\%\%\%\%\%\%\%\%\%\%\%\%\%\%\%\%\%\%\%\%\%\%\%\%\%\%\%\%\%\%\%\%\%\%\%\%\%

fid $=1 ; \quad$ \%"fid" is the file id used by the fscanf and fprintf commands

\% Note: set fid=1 to indicate the standard input/output

\% Calculate optical intensity of input and output Gaussian Beams

$\%$ and normalize the arrays

load(strcat (savelocation, '`uin'));

load(strcat (savelocation, '\posx'));

load(strcat(savelocation, '\posy'));

$\mathrm{I}=\left(\operatorname{abs}(\right.$ uin) $) \cdot \cdot^{\wedge 2} ; \quad$ \%0ptical Intensity of input beam

clear uin;

$i=\max (\max (I))$;

$\operatorname{Imax}=\mathrm{I} . / \mathrm{i}$;

$\% \quad$ clear I

\%\%\%\%\%\%\%\%\%\%\%\%\%\%\%\%\%\%\%\%\%\%\%\%\%\%\%\%\%\%\%\%\%\%\%\%\%\%\%\%\%

$\%$ Calculate the amount of power in the input beam \%

\%\%\%\%\%\%\%\%\%\%\%\%\%\%\%\%\%\%\%\%\%\%\%\%\%\%\%\%\%\%\%\%\%\%\%\%\%\%\%\%

$\operatorname{TPIn}=(\operatorname{sum}(\operatorname{sum}(I))) *(2 *$ lensradii $) \wedge 2 ;$

fprintf(fid, 'Power of input beam $=\% 2.4 \mathrm{e} \backslash \mathrm{n}^{\prime}$, TPIn);

$\%$ Plots

$\operatorname{scrsz}=\operatorname{get}\left(\odot,{ }^{\prime}\right.$ screensize' $)$;

$\%$ Sets plot window to close to full size

figure('Position', [scrsz(1)+4 scrsz(2) scrsz(3) scrsz(4)-45], 'color', [1 1 1])

mesh (posx, posy, I);

$\%$ axis('square');

axis tight;

view $(\odot, 0)$;

$\%$ sets the fontweight and fontsize for current plot)

set (gca, 'fontweight', 'demi', 'fontsize',10);

title(\{['Input beam (resolution $=$ ', num2str(delta, '\%1.2f'), 'um, lambda $=1 .$.

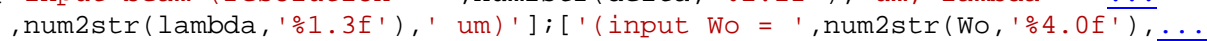

xlabel('X (um)')

ylabel('Y (um)');

zlabel('Normalized Intensity');

filename=strcat (spl, '\IWo', num2str(Wo), 'umWz', num2str(z, '\%4. ○f' ), ...

'umD', num2str(delta, '\%1.2f'), 'um.fig');

saveas (gcf, filename) 


\section{A.16 CLPlotDepth.m}

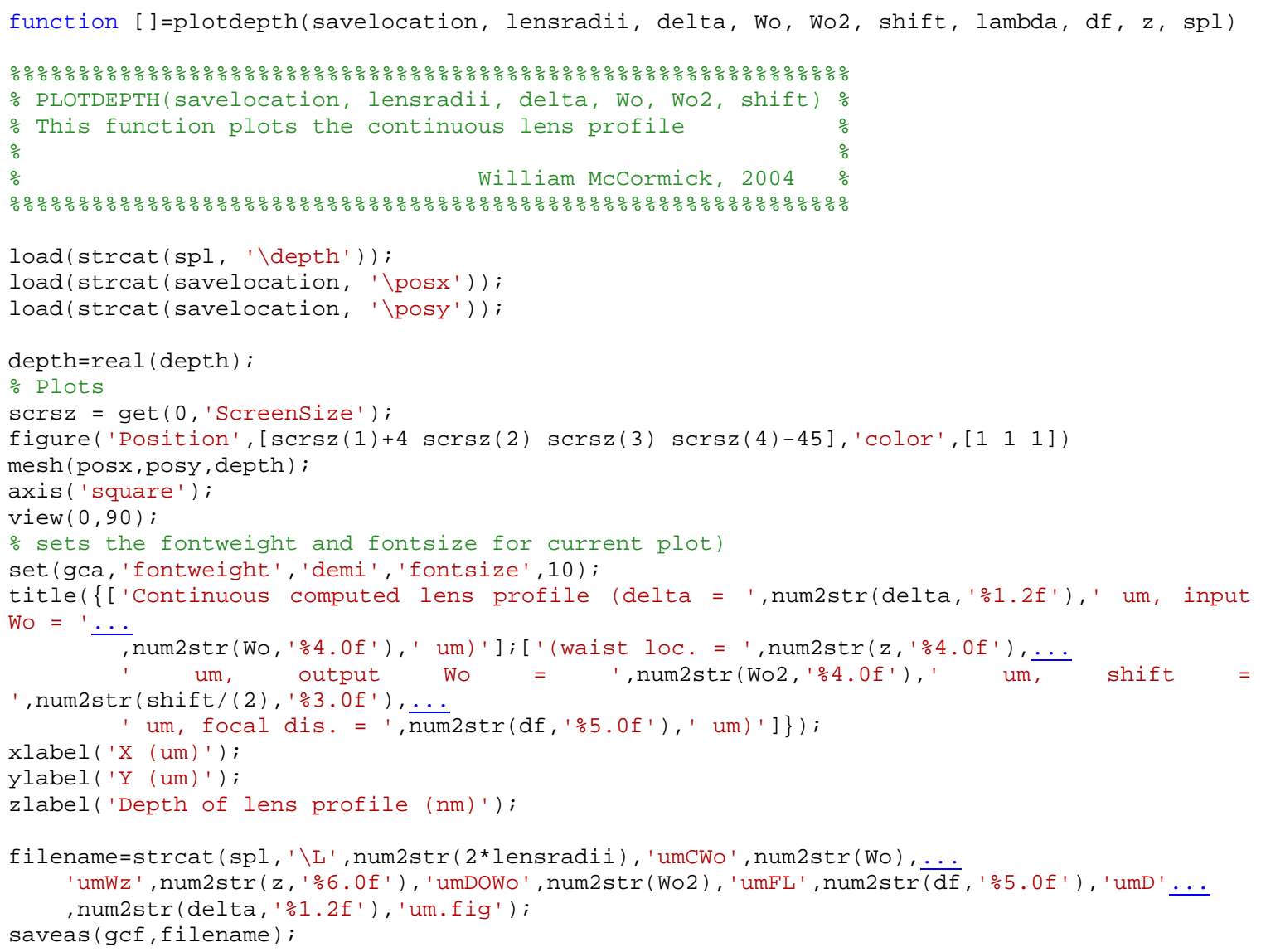




\section{Appendix B: Lens Testing Programs}

\section{B.1 TestLens.m}

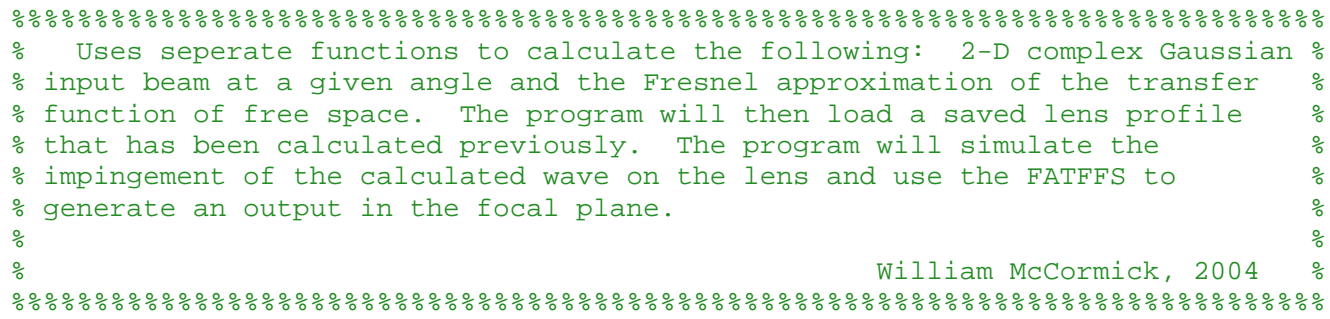

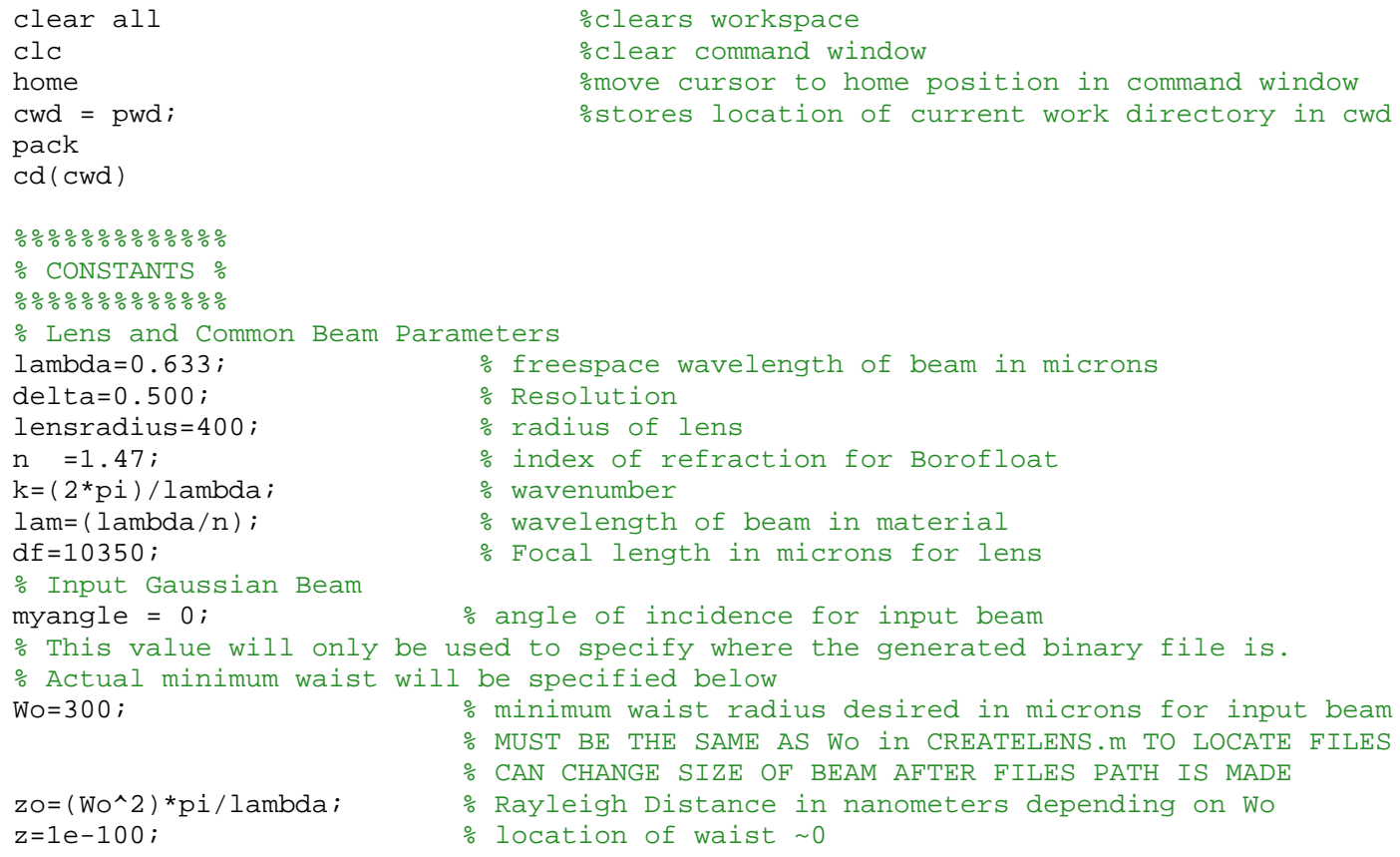


$\%$ Output Gaussian Beam Wo2=5;

$\mathrm{zo} 2=(\mathrm{pi} *(\mathrm{~W}, 2) \wedge 2) / \operatorname{lambda} ;$

\%\%\%\%\%\%\%\%\%\%\%\%\%\%\%\%\%\%\%\%\%\%\%\%\%\%\%\%\%\%\%\%\%\%\%\%\%\%\%\%\%\%\%\%\%\%\%\%\%\%\%\%\%\%\%\%\%

$\%$ "LensNumber" determines the number of lenses that the initial beam $\%$

$\% \quad$ is propogating through.

\%\%\%\%\%\%\%\%\%\%\%\%\%\%\%\%\%\%\%\%\%\%\%\%\%\%\%\%\%\%\%\%\%\%\%\%\%\%\%\%\%\%\%\%\%\%\%\%\%\%\%\%\%\%\%\%\%\%

LensNumber $=1$;

\%\%\%\%\%\%\%\%\%\%\%\%\%\%\%\%\%\%\%\%\%\%\%\%\%\%\%\%\%\%\%\%\%\%\%\%\%\%\%\%\%\%\%\%\%\%\%\%\%\%\%\%\%\%

$\%$ "Shift" is the desired distance in 1 micron increments between \%

$\%$ multiple focal points in the $x$ axis.

$\%$ "Primaryshift" is the desired shift in the output.

$\%$ "N" is the number of focal points desired.

Primaryshift $=00$

$\mathrm{N}=1$;

\%\%\%\%\%\%\%\%\%\%\%\%\%\%\%\%\%\%\%\%\%\%\%\%\%\%\%\%\%\%\%\%\%\%\%\%\%\%\%\%\%\%\%\%\%\%\%\%\%\%\%\%\%

$\%$ "FSDX" is the distance in microns the center of the input \%

$\%$ beam will be shifted away from on-axis in the x direction. \%

$\%$ "FSDy" is the distance in microns the center of the input \%

$\%$ beam will be shifted away from on-axis in the y direction. \%

\%\%\%\%\%\%\%\%\%\%\%\%\%\%\%\%\%\%\%\%\%\%\%\%\%\%\%\%\%\%\%\%\%\%\%\%\%\%\%\%\%\%\%\%\%\%\%\%\%\%\%\%\%\%\%

$\mathrm{FSDx}=000$;

$\mathrm{FSDy}=\odot \odot \odot$;

\%\%\%\%\%\%\%\%\%\%\%\%\%\%\%\%\%\%\%\%\%\%\%\%\%\%\%\%\%\%\%\%\%\%\%\%\%\%\%

$\%$ LOCATION OF SAVED FILES FROM CREATE LENS PROGRAM \%

\%\%\%\%\%\%\%\%\%\%\%\%\%\%\%\%\%\%\%\%\%\%\%\%\%\%\%\%\%\%\%\%\%\%\%\%\%\%

sl=strcat ('\Delta', num2str (delta), ' um\Lens', num2str $(2 *$ lensradius), ...

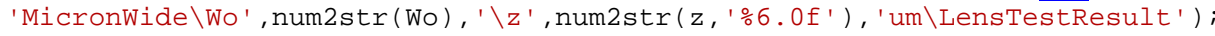

savelocation=strcat $(\mathrm{cd}, \mathrm{sl})$;

$\mathrm{ff}=$ isdir (savelocation);

if $\mathrm{ff}==0$

$\%$ if it doesn't exist, create the directory

end status=mkdir $(c d, s l)$;

loadlocation=( strcat (cd, '\Delta', num2str (delta), 'um \Lens', num2str...

(2*lensradius), 'MicronWide $\backslash W o '$, num2str(Wo $), ' \backslash z$ ', num2str...

$\left(z, ' \% 6 . \circ f^{\prime}\right)$, 'um '$\left.\left.^{\prime}\right)\right)$;

load(strcat(loadlocation, '\PrimaryTdb'));

\% \%\%\%\%\%\%\%\%\%\%\%\%\%\%\%\%\%\%\%\%\%\%\%\%\%\%\%\%\%\%\%\%\%\%\%\%\%\%\%\%\%\%\%\%\%\%\%\%\%\%\%\%\%\%\%\%\%\%\%\%\%\%\%

$\% \%$ to reduce size of lens from 400 micron radius to 300 micron radius of $0.5 \mathrm{um} \%$

$\% \%$ resolution. This will also remove noise for single spot on-axis case

$\%$

\% \%\%\%\%\%\%\%\%\%\%\%\%\%\%\%\%\%\%\%\%\%\%\%\%\%\%\%\%\%\%\%\%\%\%\%\%\%\%\%\%\%\%\%\%\%\%\%\%\%\%\%\%\%\%\%\%\%\%\%\%\%\%\%

$\% \operatorname{tdb}=\operatorname{tdb}(200: 1399,200: 1399)$;

\% \%\%\%\%\%\%\%\%\%\%\%\%\%\%\%\%\%\%\%\%\%\%\%\%\%\%\%\%\%\%\%\%\%\%\%\%\%\%\%\%\%\%\%\%\%\%\%\%\%\%\%\%\%\%\%\%\%\%\%\%\%\%\%

$\% \%$ to reduce size of lens from 400 micron radius to 300 micron radius of 0.5 um \%

$\% \%$ resolution. This will also remove noise for single spot on-axis case with \%

$\% \%$ output shifted 200 microns down on the y axis

\% \%\%\%\%\%\%\%\%\%\%\%\%\%\%\%\%\%\%\%\%\%\%\%\%\%\%\%\%\%\%\%\%\%\%\%\%\%\%\%\%\%\%\%\%\%\%\%\%\%\%\%\%

$\%$ top $=\operatorname{tdb}(1236: 1600,1: 1600)$;

$\%$ noise $=\operatorname{tdb}(1165: 1235,1: 1600)$

$\%$ bottom=tdb(1:1164,1:1600);

$\% \mathrm{tdb}=[$ bottom; top];

$\% \mathrm{tdb}=[\mathrm{tdb} ;$ noise $]$;

$\% \operatorname{tdb}=\operatorname{tdb}(200: 1399,200: 1399)$;

\% \%\%\%\%\%\%\%\%\%\%\%\%\%\%\%\%\%\%\%\%\%\%\%\%\%\%\%\%\%\%\%\%\%\%\%\%\%\%\%\%\%\%\%\%\%\%\%\%\%\%\%\%\%\%\%\%\%\%\%\%\%\%\%\%

$\% \% \quad$ This will remove noise for on-axis case with output shifted $300 \quad \%$

$\% \%$ microns down on the $y$ axis

\% \%\%\%\%\%\%\%\%\%\%\%\%\%\%\%\%\%\%\%\%\%\%\%\%\%\%\%\%\%\%\%\%\%\%\%\%\%\%\%\%\%\%\%\%\%\%\%\%\%\%\%\%\%\%\%\%\%\%

$\%$ top $=\operatorname{tdb}(1036: 1600,1: 1600)$;

$\%$ noise $=\operatorname{tdb}(970: 1035,1: 1600)$

$\%$ bottom $=\operatorname{tdb}(1: 969,1: 1600)$; 
$\%$ tdb=[bottom; top];

$\% \mathrm{tdb}=[\mathrm{tdb} ;$ noise $]$;

$\% \% \operatorname{tdb}=\operatorname{tdb}(1: 1535,32: 1566)$;

\% \%\%\%\%\%\%\%\%\%\%\%\%\%\%\%\%\%\%\%\%\%\%\%\%\%\%\%\%\%\%\%\%\%\%\%\%\%\%\%\%\%\%\%\%\%\%\%\%\%\%\%\%\%\%\%\%\%

$\% \%$ This will remove noise for on-axis case with dual output shifted $300 \%$

$\% \%$ microns down on the $y$ axis for lambda $=1.310$ and resolution of $0.5 \mathrm{um} . \%$

\% \%\%\%\%\%\%\%\%\%\%\%\%\%\%\%\%\%\%\%\%\%\%\%\%\%\%\%\%\%\%\%\%\%\%\%\%\%\%\%\%\%\%\%\%\%\%\%\%\%\%\%\%\%\%\%\%\%\%\%

$\%$ top $=\operatorname{tdb}(1034: 1600,85: 1515)$;

$\%$ bottom=tdb $(1: 969,85: 1515)$;

$\% \operatorname{tdb}=[$ bottom; top];

\% \%\%\%\%\%\%\%\%\%\%\%\%\%\%\%\%\%\%\%\%\%\%\%\%\%\%\%\%\%\%\%\%\%\%\%\%\%\%\%\%\%\%\%\%\%\%\%\%\%\%\%\%\%\%\%\%

$\% \%$ This will remove noise for on-axis case with dual output shifted $300 \%$

$\% \%$ microns down on the $y$ axis for lambda $=0.633$ and resolution of $0.5 \mathrm{um} . \%$

\% \%\%\%\%\%\%\%\%\%\%\%\%\%\%\%\%\%\%\%\%\%\%\%\%\%\%\%\%\%\%\%\%\%\%\%\%\%\%\%\%\%\%\%\%\%\%\%\%\%\%\%\%\%\%\%\%\%

$\%$ top $=\operatorname{tdb}(1035: 1600,36: 1564)$

$\%$ bottom=tdb $(1: 969,36: 1564)$;

$\%$ tdb=[bottom; top];

\% \%\%\%\%\%\%\%\%\%\%\%\%\%\%\%\%\%\%\%\%\%\%\%\%\%\%\%\%\%\%\%\%\%\%\%\%\%\%\%\%\%\%\%\%\%\%\%\%\%\%\%\%\%\%\%\%\%\%\% $\% \%$ This will remove noise for on-axis case with single output shifted $300 \%$

$\% \%$ microns down on the $y$ axis for lambda $=0.633$ and resolution of $0.5 \mathrm{um} . \%$ $\%$ SLIGHTLY OFFCENTER

\% \%\%\%\%\%\%\%\%\%\%\%\%\%\%\%\%\%\%\%\%\%\%\%\%\%\%\%\%\%\%\%\%\%\%\%\%\%\%\%\%\%\%\%\%\%\%\%\%\%\%\%\%\%\%\%\%\%\%\%\%\%

$\%$ top $=\operatorname{tdb}(1030: 1600,32: 1568)$

$\%$ bottom $=\operatorname{tdb}(1: 974,32: 1568)$;

$\% \mathrm{tdb}=[$ bottom; top];

\% \%\%\%\%\%\%\%\%\%\%\%\%\%\%\%\%\%\%\%\%\%\%\%\%\%\%\%\%\%\%\%\%\%\%\%\%\%\%\%\%\%\%\%\%\%\%\%\%\%\%\%\%\%\%\%\%\%\%

$\% \%$ This will remove noise for on-axis case with single output for lambda \%

$\%$ eqaul to 0.633 and resolution of 0.5 um. $\%$

$\% \%$ MESSED UP SINGLE ON LENS $5 \mathrm{~mm}$

\% \%\%\%\%\%\%\%\%\%\%\%\%\%\%\%\%\%\%\%\%\%\%\%\%\%\%\%\%\%\%\%\%\%\%\%\%\%\%\%\%\%\%\%\%\%\%\%\%\%\%\%\%\%\%\%\%\%\%

$\%$ top=tdb $(1030: 1600,36: 1564)$;

$\%$ bottom=tdb $(1: 970,36: 1564)$;

$\%$ tdb=[bottom; top];

\% \%\%\%\%\%\%\%\%\%\%\%\%\%\%\%\%\%\%\%\%\%\%\%\%\%\%\%\%\%\%\%\%\%\%\%\%\%\%\%\%\%\%\%\%\%\%\%\%\%\%\%\%\%\%\%\%\%\%

$\% \%$ This will remove noise for on-axis case with dual output for lambda \%

$\% \%$ eqaul to 0.633 and resolution of 0.5 um.

\% \%\%\%\%\%\%\%\%\%\%\%\%\%\%\%\%\%\%\%\%\%\%\%\%\%\%\%\%\%\%\%\%\%\%\%\%\%\%\%\%\%\%\%\%\%\%\%\%\%\%\%\%\%\%\%\%\%\%\%

$\% \operatorname{tdb}=\operatorname{tdb}(30: 1570,80: 1520)$;

\%\%\%\%\%\%\%\%\%\%\%\%\%\%\%\%\%\%\%\%\%\%\%\%\%\%\%\%\%\%\%\%\%\%\%\%\%\%\%\%\%\%\%\%\%\%\%\%\%\%\%\%\%\%\%\%\%\%\%\%\%\%\%\%\%\%

\%AUGUST 2004

\% \%\%\%\%\%\%\%\%\%\%\%\%\%\%\%\%\%\%\%\%\%\%\%\%\%\%\%\%\%\%\%\%\%\%\%\%\%\%\%\%\%\%\%\%\%\%\%\%\%\%\%\%\%\%\%\%

$\% \%$ This will remove noise for on-axis case single output for lambda=633nm \%

$\% \%$ for lens generated with 300 um input beam, resolution of 0.5 um and focal\%

$\% \%$ length of $5 \mathrm{~mm}$.

\% \%\%\%\%\%\%\%\%\%\%\%\%\%\%\%\%\%\%\%\%\%\%\%\%\%\%\%\%\%\%\%\%\%\%\%\%\%\%\%\%\%\%\%\%\%\%\%\%\%\%\%\%\%\%\%\%\%\%\%

$\% \operatorname{tdb}=\operatorname{tdb}(60: 1540,60: 1540)$;

\%\%\%\%\%\%\%\%\%\%\%\%\%\%\%\%\%\%\%\%\%\%\%\%\%\%\%\%\%\%\%\%\%\%\%\%\%\%\%\%\%\%\%\%\%\%\%\%

\% Saves the lens file after noise regions have been removed. \%

$\%$ Also saves the new value for the lens radius that is equal \%

$\%$ to the size of the new lens file. Initial value of lens- \%

$\%$ radii is used to locate initial lens file.

\%\%\%\%\%\%\%\%\%\%\%\%\%\%\%\%\%\%\%\%\%\%\%\%\%\%\%\%\%\%\%\%\%\%\%\%\%\%\%\%\%\%\%\%\%\%\%\%

save((strcat (savelocation, '\PrimaryTdb.mat')), 'tdb');

load(strcat (savelocation, '\PrimaryTdb'));

lensradius $=\operatorname{delta*}(($ length $(\mathrm{tdb})) / 2)$;

save((strcat(savelocation, '\lensradius.mat')), 'lensradius');

\%\%\%\%\%\%\%\%\%\%\%\%\%\%\%\%\%\%\%\%\%\%\%\%\%\%\%\%\%

$\%$ create $\mathrm{x}$ and $\mathrm{y}$ vectors for plots \% \%\%\%\%\%\%\%\%\%\%\%\%\%\%\%\%\%\%\%\%\%\%\%\%\%\%\%\%\%

right $=$ lensradius; 


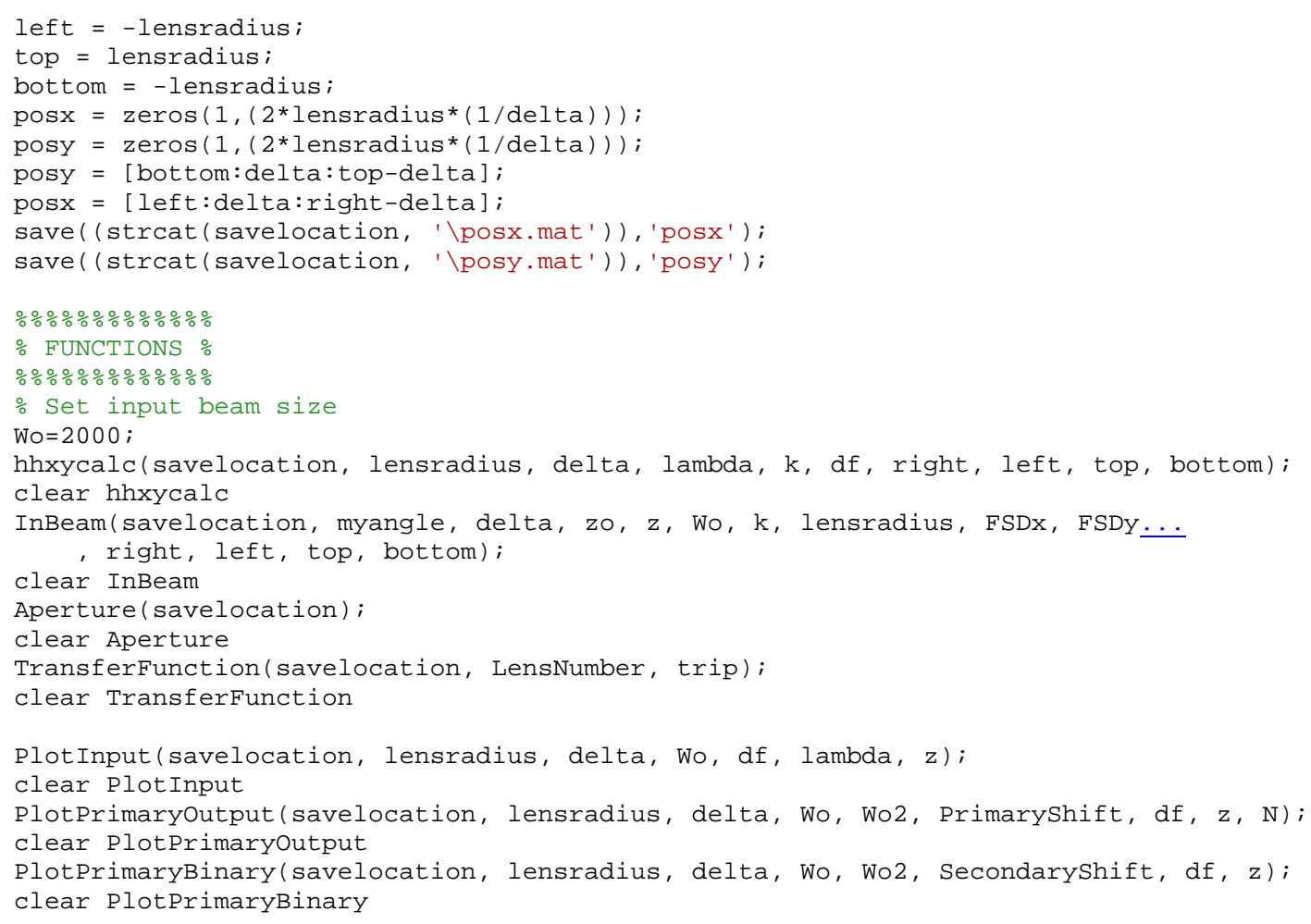




\section{B.3 Inbeam.m}

function []=inbeam(savelocation, myangle, delta, zo, z, Wo, k, lensradius, FSDx, FSDy,... right, left, top, bottom) \%\%\%\%\%\%\%\%\%\%\%\%\%\%\%\%\%\%\%\%\%\%\%\%\%\%\%\%\%\%\%\%\%\%\%\%\%\%\%\%\%\%\%\%\%\%\%\%\%\%\%\%\%\%\%\%\%\%\%\%\%\%\%\% \% INBEAM(savelocation, myabgle, delta, zo, z, Wo, k, lambda, lensradii, FSDx, FSDy, \% $\%$ right, left, top, bottom)

\% Calculates the 2-D complex Gaussian input beam using parameters for loop $\%$

\% size(lensradii), loop resolution(delta), Rayleigh range of input beam(zo), location \%

$\%$ of output beam waist in relation to $z$ axis(z), minimum waist radius desired for $\%$

$\%$ input beam(Wo) and the wavenumber(k). Function then saves Gaussian array to file \%

$\%$ Primaryinput.mat, phase information(bp.mat), posx.mat and posy (posx and posy \%

$\%$ allows plot to range from -size to size in x-direction) $\%$

$\% \quad$ William McCormick, $20 \odot 4 \quad \%$

\%\%\%\%\%\%\%\%\%\%\%\%\%\%\%\%\%\%\%\%\%\%\%\%\%\%\%\%\%\%\%\%\%\%\%\%\%\%\%\%\%\%\%\%\%\%\%\%\%\%\%\%\%\%\%\%\%\%\%\%\%\%\%\%\%\%\%\%\%\%

fid $=1$;

\%\%\%\%\%\%\%\%\%\%\%\%\%\%\%\%\%\%\%\%\%\%\%\%\%\%\%\%\%\%\%\%\%\%\%\%\%\%\%\%\%\%\%\%\%\%\%\%\%

$\%$ "fid" is the file id used by the fscanf and fprintf commands.\%

$\%$ Note: set fid=1 to indicate the standard input/output.

\%\%\%\%\%\%\%\%\%\%\%\%\%\%\%\%\%\%\%\%\%\%\%\%\%\%\%\%\%\%\%\%\%\%\%\%\%\%\%\%\%\%\%\%\%\%\%\%\%\%\%\%\%

fprintf (fid, '\nInput beam calculation\n');

\%\%\%\%\%\%\%\%\%\%\%\%\%\%\%\%\%\%\%\%\%\%\%\%\%\%\%\%\%\%

$\%$ DEFINE VECTORS, MATRICES and ARRAYS \%

$\% \% \% \% \% \% \% \% \% \% \% \% \% \% \% \% \% \% \% \% \% \% \% \% \% \% \%$

theta=myangle * $\mathrm{pi} / 180$;

\%\%\%\%\%\%\%\%\%\%\%\%\%\%\%\%\%\%\%\%\%\%\%\%\%\%\%\%\%\%\%\%\%

\% 2-D COMPLEX GAUSSIAN BEAM AMPLITUDE U(x) \%

\%\%\%\%\%\%\%\%\%\%\%\%\%\%\%\%\%\%\%\%\%\%\%\%\%\%\%\%\%\%\%\%\%\%\%\%

ticker $=0$;

tic

elapsedtime $=[]$;

count $=\odot$

ur $=$ zeros $((2 *$ lensradius* $(1 /$ delta $)),(2 *$ lensradius* $(1 /$ delta $)))$;

\%\%\%\%\%\%\%\%\%\%\%\%\%\%

\% Calculate Beam \%

$\% \% \% \% \% \% \% \% \% \% \% \%$

for $x=$ bottom-FSDx: delta: top-FSDx-delta

$\%$ Convert from $x$ and $z$ orientation to xprime and zprime

$\%$ also convert zo (Rayleigh range of beam) to zoprime

xprime $=\left(x^{*} \cos (\right.$ theta $\left.)\right)-\left(z{ }^{*} \sin (\right.$ theta $\left.)\right)$;

zprime $=\operatorname{sqrt}\left(x^{\wedge} 2+z^{\wedge} 2-x p r i m e \wedge 2\right) ;$

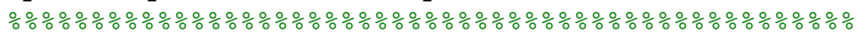

\% Make sure that we have no divide by zero issues \% \%\%\%\%\%\%\%\%\%\%\%\%\%\%\%\%\%\%\%\%\%\%\%\%\%\%\%\%\%\%\%\%\%\%\%\%\%\%\%\%\% if zprime $==0$

end

zprime=1e-320;

zoprime=zo;

$\mathrm{c}=\odot$;

count $=$ count +1

$\mathrm{R}=\mathrm{rem}$ (count, 100$)$;

ticker $=$ ticker +1 ;

if $R==0$ end

fprintf(fid, 'Count $=\% 4.0 f$, Time $=\% 6.2 f$ secs $\backslash n \backslash$ ', count, toc $)$;

for $y=l e f t-F S D y:$ delta:right-FSDy-delta

$\mathrm{c}=\mathrm{c}+1$;

yprime=y;

psq=xprime^2+yprime^2

$\mathrm{Wz}=W \mathrm{~W}^{*} \operatorname{sqrt}\left(1+(\mathrm{zprime} / \text { zoprime })^{\wedge} 2\right)$;

$\mathrm{Rz}=\mathrm{z}^{*}\left(1+(\text { zoprime/zprime })^{\wedge} 2\right) ;$

Sz=atan (zprime/zoprime);

bphase $=\left(\left(k^{*} z p r i m e\right)-\left(k^{*}(p s q /(2 * R z))\right)+S z\right)$;

$\mathrm{u}=\left(1 /\left(j^{*} \text { zoprime }\right)\right)^{*}(\mathrm{Wo} / \mathrm{Wz})^{*} \exp \left(-\mathrm{psq} /\left(\mathrm{W} z^{\wedge} 2\right)\right)^{*} \exp \left(-j^{*}\right.$ bphase $)$;

end

$\operatorname{ur}($ count,$c)=u$

end 
\%\%\%\%\%\%\%\%\%\%\%\%\%\%\%\%\%\%\%\%\%\%\%\%\%\%\%\%\%\% $\%$ SAVE ARRAYS FOR FUTURE MANIPULATION \% \%\%\%\%\%\%\%\%\%\%\%\%\%\%\%\%\%\%\%\%\%\%\%\%\%\%\%\%\%\%\% tempvar = '\PrimaryInput . mat' ';

saveur=strcat (savelocation, tempvar);

save( saveur, 'ur');

elapsedtime $=[$ elapsedtime; toc $]$

total=( sum(elapsedtime $))$;

fprintf(fid,'Total time for input beam calculation: \%6.2f secs $\backslash n \backslash n$ ', total); 


\section{B.4 Aperture.m}

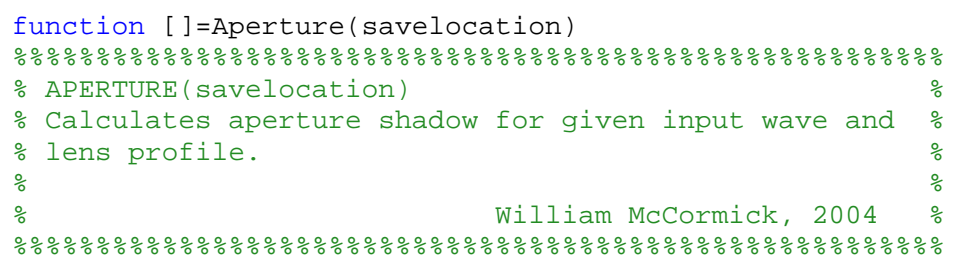

fid = 1; $\quad$ \%"fid" is the file id used by the fscanf and fprintf commands \% Note: set fid=1 to indicate the standard input/output

fprintf (fid, 'Primary aperture output calculation \n'); load(strcat(savelocation, '\PrimaryTdb'));

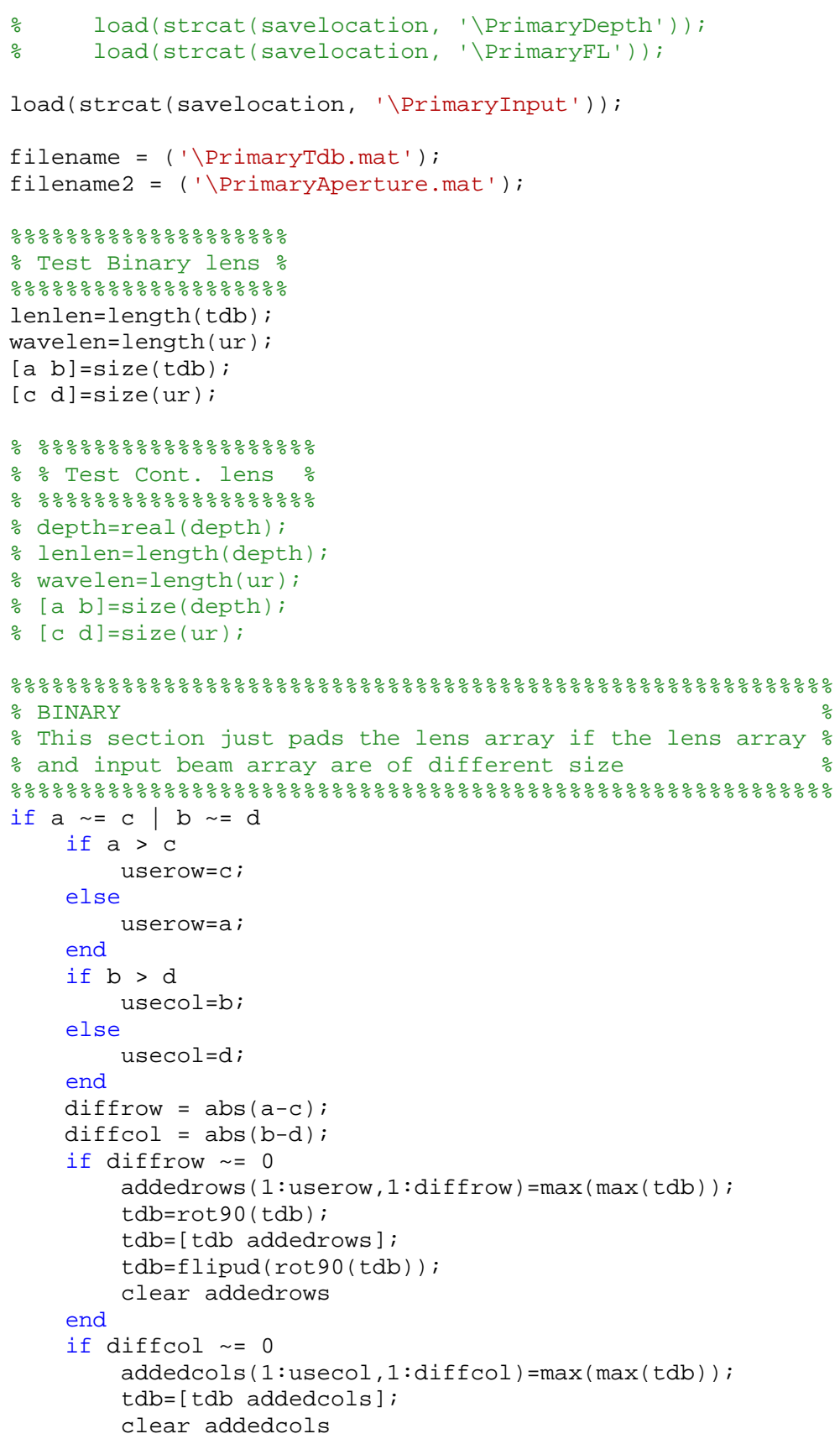




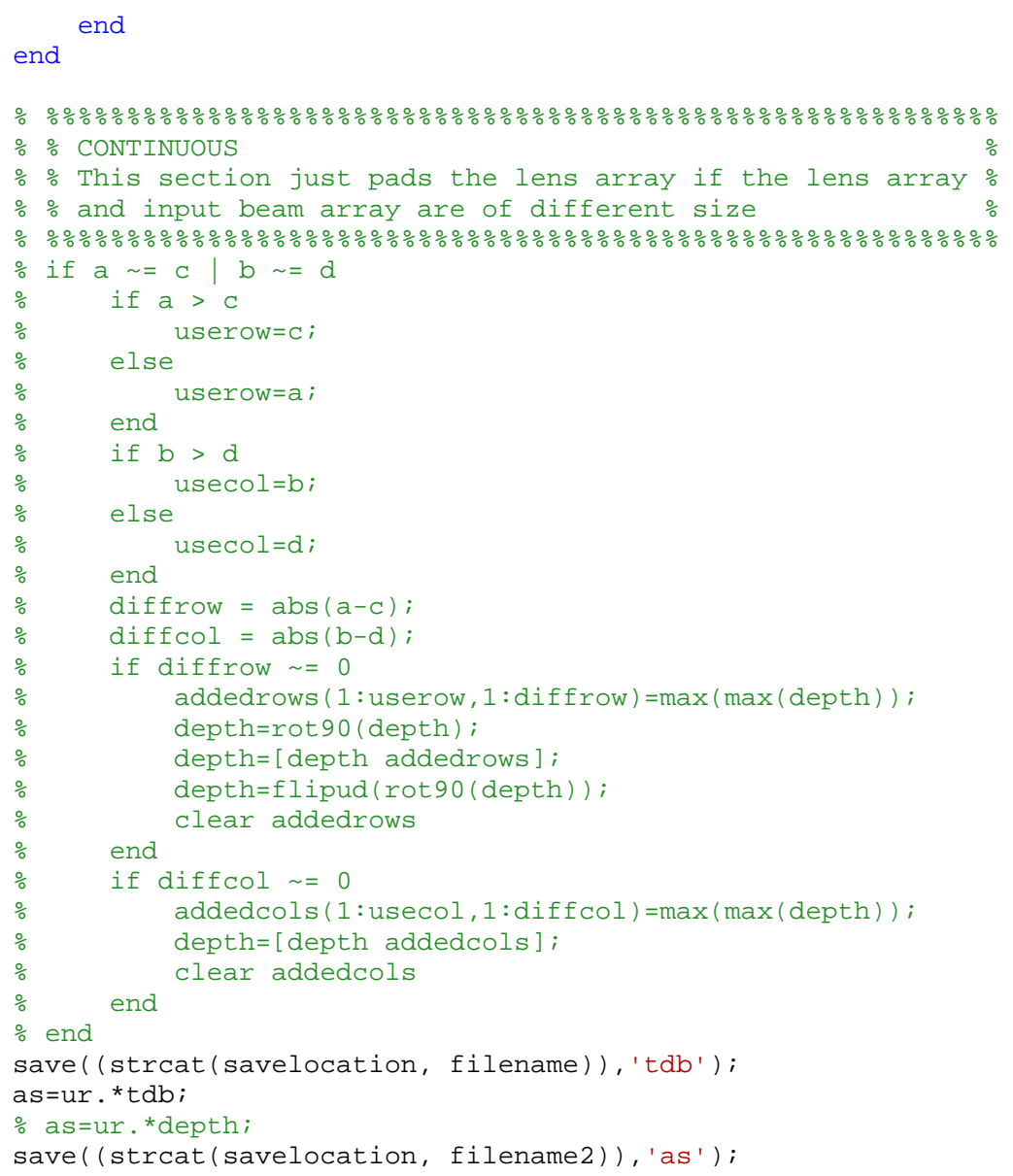




\section{B.5 TransferFunction.m}

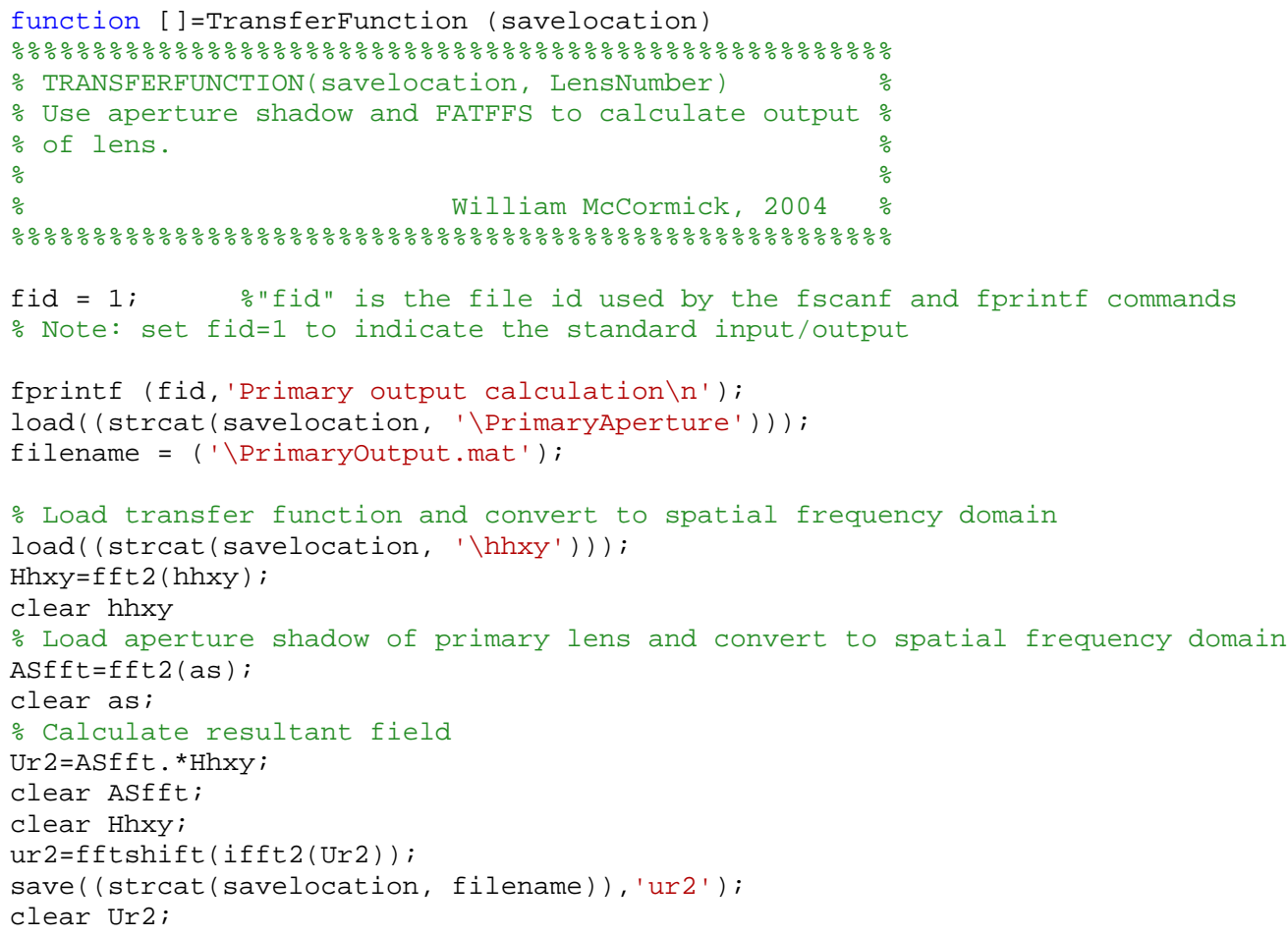




\section{B.6 PlotInput.m}

function []=PlotInput(savelocation, lensradius, delta, Wo, df, lambda, z) \%\%\%\%\%\%\%\%\%\%\%\%\%\%\%\%\%\%\%\%\%\%\%\%\%\%\%\%\%\%\%\%\%\%\%\%\%\%\%\%\%\%\%\%\%\%\%\%\%\%\%\%\%\%\%\%\%\%\% \% PLOTINPUT(savelocation, lensradius, delta, Wo, Wo2, df, lambda, z) \% \% Import input complex Gaussian Beam file and plot the intensity profile. \% $\%$ Uses lensradius to determine plot axis and the variables Wo, df, lambda, \% $\%$ and $z$ for identification in the title. $\%$ William McCormick, 2004 \%\%\%\%\%\%\%\%\%\%\%\%\%\%\%\%\%\%\%\%\%\%\%\%\%\%\%\%\%\%\%\%\%\%\%\%\%\%\%\%\%\%\%\%\%\%\%\%\%\%\%\%\%\%\%\%\%\%\%\%\%\%\%

fid $=1 ; \quad$ \%"fid" is the file id used by the fscanf and fprintf commands \% Note: set fid=1 to indicate the standard input/output

\% Calculate optical intensity of input and output Gaussian Beams

$\%$ and normalize the arrays

load(strcat (savelocation, '\PrimaryInput'));

$\mathrm{I}=(\operatorname{abs}(\mathrm{ur})) . \wedge 2$; $\quad$ \%optical Intensity of input beam

clear ur:

$i=\max (\max (I))$;

$\operatorname{Imax}=\mathrm{I} . / \mathrm{i}$;

$\% \quad$ clear I

\%\%\%\%\%\%\%\%\%\%\%\%\%\%\%\%\%\%\%\%\%\%\%\%\%\%\%\%\%\%\%\%\%\%\%\%\%\%\%\%\%

\% Calculate the amount of power in the input beam \%

\%\%\%\%\%\%\%\%\%\%\%\%\%\%\%\%\%\%\%\%\%\%\%\%\%\%\%\%\%\%\%\%\%\%\%\%\%\%\%\%

$\operatorname{TPIn}=(\operatorname{sum}(\operatorname{sum}(I))) *(2 *$ lensradius $) \wedge 2$;

fprintf(fid, 'Power of input beam $=\% 2.4 \mathrm{e} \backslash \mathrm{n}$ ', TPIn);

save(( strcat(savelocation, '\TPIn.mat')), 'TPIn');

load(strcat (savelocation, '\posx'));

load(strcat(savelocation, '\posy'));

$\%$ Plots

scrsz $=\operatorname{get}\left(\odot,{ }^{\prime}\right.$ Screensize' $)$;

$\%$ Sets plot window to close to full size

figure('Position', [scrsz(1)+4 scrsz(2) scrsz(3) scrsz(4)-45], 'color', [1 11 1])

mesh(posx, posy, I, 'FaceColor', 'flat',...

'FaceLighting', 'phong', 'Linestyle', 'none', 'EdgeLighting', 'phong' );

axis('square');

view $(\odot, 9 \odot)$; grid off;

\% \%\%\%\%\%\%\%\%\%\%\%\%\%\%\%\%\%\%\%\%\%\%\%\%\%\%\%\%\%\%\%\%\%\%\%\%\%\%\%\%\%\%\%\%\%\%\%\%\%\%\%\%\%\%\%\%\%\%\%\%\%\%\%\%\%\% $\% \%$ sets the fontweight and fontsize for current plot for a 3 inch wide picture in word \% \% \%\%\%\%\%\%\%\%\%\%\%\%\%\%\%\%\%\%\%\%\%\%\%\%\%\%\%\%\%\%\%\%\%\%\%\%\%\%\%\%\%\%\%\%\%\%\%\%\%\%\%\%\%\%\%\%\%\%\%\%\%\%\%\%\%\% $\% \operatorname{set}($ gca, 'fontweight ', 'demi', 'fontsize', 18);

\% \%\%\%\%\%\%\%\%\%\%\%\%\%\%\%\%\%\%\%\%\%\%\%\%\%\%\%\%\%\%\%\%\%\%\%\%\%\%\%\%\%\%\%\%\%\%\%\%\%\%\%\%\%\%\%\%\%\%\%\%\%\%\%\%\% $\% \%$ sets the fontweight and fontsize for current plot for a 5 inch wide picture in word \% \% \%\%\%\%\%\%\%\%\%\%\%\%\%\%\%\%\%\%\%\%\%\%\%\%\%\%\%\%\%\%\%\%\%\%\%\%\%\%\%\%\%\%\%\%\%\%\%\%\%\%\%\%\%\%\%\%\%\%\%\%\%\%\%\%\%\%\%\%

$\% \operatorname{set}($ gca, 'fontweight', 'demi', 'fontsize',14);

\%\%\%\%\%\%\%\%\%\%\%\%\%\%\%\%\%\%\%\%\%\%\%\%\%\%\%\%\%\%\%\%\%\%\%\%\%\%\%\%\%\%\%\%\%\%\%\%\%\%\%\%\%\%\%\%\%\%\%\%\%\%\%\%\%\%\%\% $\%$ sets the fontweight and fontsize for current plot for a 6 inch wide picture in word \% \%\%\%\%\%\%\%\%\%\%\%\%\%\%\%\%\%\%\%\%\%\%\%\%\%\%\%\%\%\%\%\%\%\%\%\%\%\%\%\%\%\%\%\%\%\%\%\%\%\%\%\%\%\%\%\%\%\%\%\%\%\%\%\%\%\%\%\% set(gca, 'fontweight', 'demi', 'fontsize', 10);

title(\{['Input beam (delta $=$ ', num2str(delta, '\%1.2f'), 'um, lambda $=$ '...

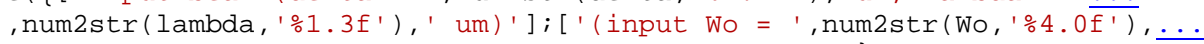
' um, waist location $\left.\left.\left.\left.={ }^{\prime}, \operatorname{num} 2 \operatorname{str}\left(z, \% 4.0 f^{\prime}\right),{ }^{\prime} u m\right)^{\prime}\right]\right\}\right)$;

xlabel('X (um)');

ylabel('Y (um)');

zlabel('Intensity $(\mathrm{W} / \mathrm{um} \wedge 2)$ ')'

filename=strcat ( savelocation, '\IWo' , num2str(Wo), 'umWz', num2str(z, '\%6. $f^{\prime}$ '), ... 'umD', num2str(delta, '\%1.2f'), 'um.fig');

saveas (gcf, filename); 


\section{B.7 PlotPrimaryOutput.m}

function []=PlotPrimaryoutput(savelocation, lensradius, delta, Wo, Wo2, Primaryshift, df, $\mathrm{Z}, \mathrm{N})$

\%\%\%\%\%\%\%\%\%\%\%\%\%\%\%\%\%\%\%\%\%\%\%\%\%\%\%\%\%\%\%\%\%\%\%\%\%\%\%\%\%\%\%\%\%\%\%\%\%\%\%\%\%\%\%\%\%\%\%\%\%\%\%\%\%\%\%\%\%

\% PLOTPRIMARYOUTPUT(savelocation, lensradius, delta, Wo, Wo2, PrimaryShift, df, z, N) \%

\% Import primary output file from primary lens and plot the intensity profile. Uses \%

$\%$ lensradius to determine plot axis and power contained in the beam. The variables \%

$\%$ Wo, Wo2, Primaryshift, df, lambda, zo, $\mathrm{z}$ and $\mathrm{N}$ are for identification in the title. \%

$\%$

William McCormick, $2004 \%$

\%\%\%\%\%\%\%\%\%\%\%\%\%\%\%\%\%\%\%\%\%\%\%\%\%\%\%\%\%\%\%\%\%\%\%\%\%\%\%\%\%\%\%\%\%\%\%\%\%\%\%\%\%\%\%\%\%\%\%\%\%\%\%\%\%

fid $=1 ; \quad$ \%"fid" is the file id used by the fscanf and fprintf commands

$\%$ Note: set fid=1 to indicate the standard input/output

\% Calculate optical intensity of input and output Gaussian Beams

$\%$ and normalize the arrays

load(strcat(savelocation, '\Primaryoutput'));

I2= $(\operatorname{abs}(\operatorname{ur} 2)) \cdot \wedge 2 ; \quad$ \%optical intensity of output beam

clear ur2;

$i=\max (\max (I 2))$;

$\operatorname{Imax} 2=\mathrm{I} 2 . / \mathrm{i}$;

\%\%\%\%\%\%\%\%\%\%\%\%\%\%\%\%\%\%\%\%\%\%\%\%\%\%\%\%\%\%\%\%\%\%\%\%\%\%\%\%\%

\%Calculate the amount of power in the output beam \%

\%\%\%\%\%\%\%\%\%\%\%\%\%\%\%\%\%\%\%\%\%\%\%\%\%\%\%\%\%\%\%\%\%\%\%\%\%\%\%

TPOut $=(\operatorname{sum}(\operatorname{sum}($ I2 $))) *(2 *$ lensradius $) \wedge 2$;

fprintf(fid, 'Power of output beam $=\% 2.4 \mathrm{e} \backslash \mathrm{n}$ ', TPOut);

save((strcat (savelocation, '\PrimaryoutputPower.mat')), 'TPout ');

load(strcat (savelocation, '\posx'));

load(strcat(savelocation, '\posy'));

$\%$ Plots

$\operatorname{scrsz}=\operatorname{get}\left(\odot\right.$, 'Screensize' $\left.^{\prime}\right)$;

\%\%\%\%\%\%\%\%\%\%\%\%\%\%\%

$\%$ Plot angled view \%

$\% \% \% \% \% \% \% \% \% \% \% \% \%$

$\%$ sets plot window to close to full size

figure('Position', [scrsz(1)+4 scrsz(2) scrsz(3) scrsz(4)-45], 'color', [1 11 1])

\%\%\%\%\%\%\%\%\%\%\%\%\%\%\%\%\%\%\%\%\%\%\%\%\%\%\%\%\%\%\%\%\%\%\%\%\%\%\%\%\%\%\%\%

$\%$ To print just a portion of the array and save memory \%

\%\%\%\%\%\%\%\%\%\%\%\%\%\%\%\%\%\%\%\%\%\%\%\%\%\%\%\%\%\%\%\%\%\%\%\%\%\%\%\%\%

mesh (posx(700:901), posy(700:901), I2(700:901,700:901), 'FaceColor', 'interp', ...

'FaceLighting', 'phong', 'LineStyle', 'none', 'EdgeLighting', 'phong' );

axis tight;

$\operatorname{view}(45,70)$;

light('Position', [1 10 0], 'Color', [0.498 0.498 0.498], 'Style','infinite');

light('Position', [-1 -10$]$, 'Color', [0.498 0.498 0.498], 'Style', 'infinite');

light('Position', [-1 10 , 'Color', [0.498 0.498 0.498], 'Style', 'infinite');

light('Position', [1 -10$]$, 'Color', [0.498 0.498 0.498], 'Style', 'infinite');

light('Position', [ $\left.\begin{array}{lll}0 & 0 & 1\end{array}\right]$,' 'Style', 'infinite');

grid off

\%\%\%\%\%\%\%\%\%\%\%\%\%\%\%\%\%\%\%\%\%\%\%\%\%\%\%\%\%\%\%\%\%\%\%\%\%\%\%\%\%\%\%\%\%\%\%\%\%\%\%\%\%\%\%\%\%\%\%\%\%\%\%\%\%\% $\%$ sets the fontweight and fontsize for current plot for a 3 inch wide picture in word \% \%\%\%\%\%\%\%\%\%\%\%\%\%\%\%\%\%\%\%\%\%\%\%\%\%\%\%\%\%\%\%\%\%\%\%\%\%\%\%\%\%\%\%\%\%\%\%\%\%\%\%\%\%\%\%\%\%\%\%\%\%\%\%\%\%\%\%\%\%\% set (gca, ' fontweight', 'demi', ' fontsize', 18);

$\%$

\%\%\%\%\%\%\%\%\%\%\%\%\%\%\%\%\%\%\%\%\%\%\%\%\%\%\%\%\%\%\%\%\%\%\%\%\%\%\%\%\%\%\%\%\%\%\%\%\%\%\%\%\%\%\%\%\%\%\%\%\%\%\%\%\%\%\% $\%$ sets the fontweight and fontsize for current plot for a 5 inch wide picture in word \% \%\%\%\%\%\%\%\%\%\%\%\%\%\%\%\%\%\%\%\%\%\%\%\%\%\%\%\%\%\%\%\%\%\%\%\%\%\%\%\%\%\%\%\%\%\%\%\%\%\%\%\%\%\%\%\%\%\%\%\%\%\%\%\%\%\%\%\% $\% \operatorname{set}($ gca, 'fontweight', 'demi', 'fontsize',14);

\%\%\%\%\%\%\%\%\%\%\%\%\%\%\%\%\%\%\%\%\%\%\%\%\%\%\%\%\%\%\%\%\%\%\%\%\%\%\%\%\%\%\%\%\%\%\%\%\%\%\%\%\%\%\%\%\%\%\%\%\%\%\%\%\%\% $\%$ sets the fontweight and fontsize for current plot for a 6 inch wide picture in word $\%$ 
\%\%\%\%\%\%\%\%\%\%\%\%\%\%\%\%\%\%\%\%\%\%\%\%\%\%\%\%\%\%\%\%\%\%\%\%\%\%\%\%\%\%\%\%\%\%\%\%\%\%\%\%\%\%\%\%\%\%\%\%\%\%\%\%\%\%\%\% \% set (gca, 'fontweight', 'demi', 'fontsize', 10);

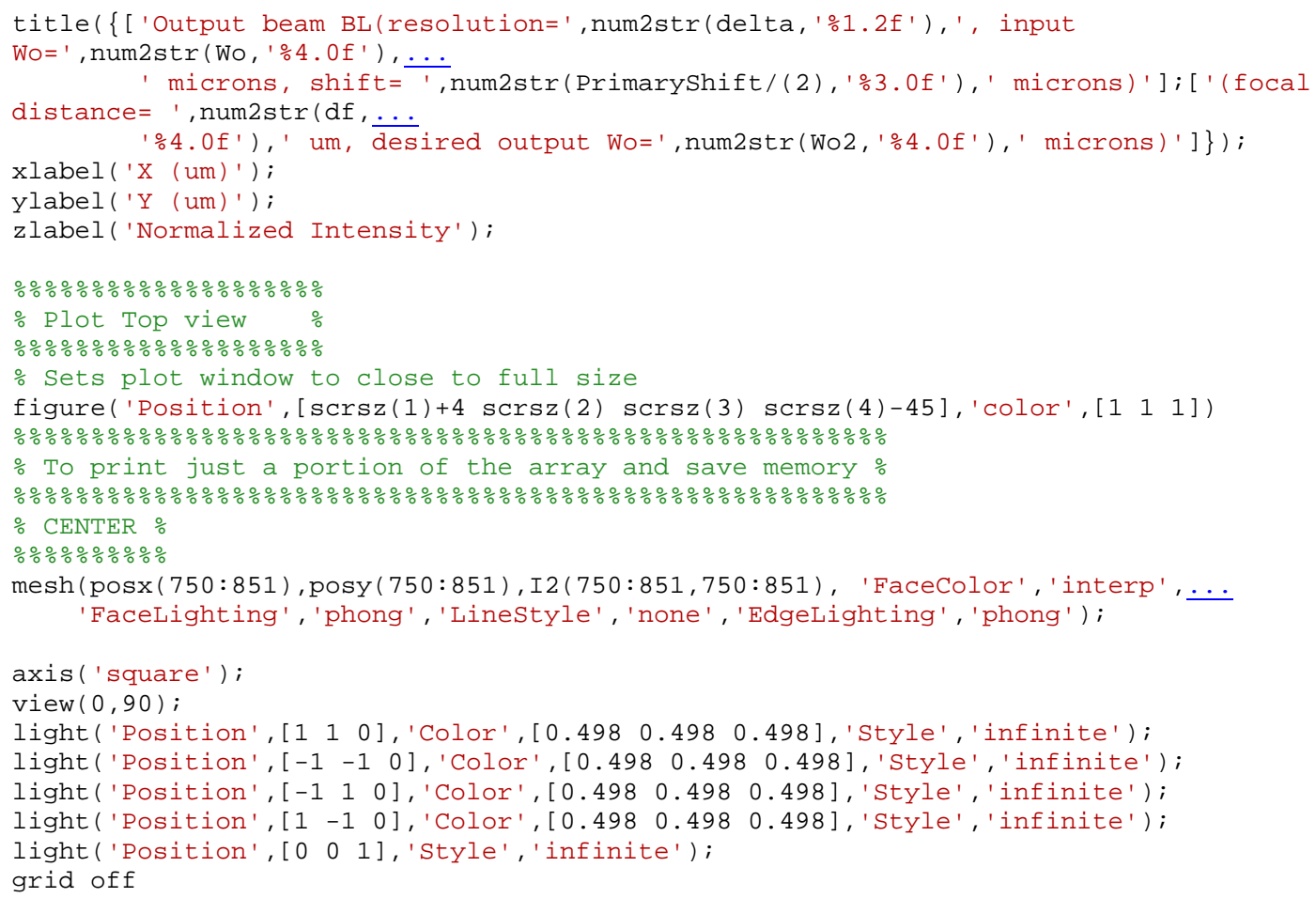

\%\%\%\%\%\%\%\%\%\%\%\%\%\%\%\%\%\%\%\%\%\%\%\%\%\%\%\%\%\%\%\%\%\%\%\%\%\%\%\%\%\%\%\%\%\%\%\%\%\%\%\%\%\%\%\%\%\%\%\%\%\%\%\%\%\%\%\% $\%$ sets the fontweight and fontsize for current plot for a 3 inch wide picture in word \% \%\%\%\%\%\%\%\%\%\%\%\%\%\%\%\%\%\%\%\%\%\%\%\%\%\%\%\%\%\%\%\%\%\%\%\%\%\%\%\%\%\%\%\%\%\%\%\%\%\%\%\%\%\%\%\%\%\%\%\%\%\%\%\%\%\%\% set (gca, 'fontweight', 'demi', 'fontsize',18);

\%\%\%\%\%\%\%\%\%\%\%\%\%\%\%\%\%\%\%\%\%\%\%\%\%\%\%\%\%\%\%\%\%\%\%\%\%\%\%\%\%\%\%\%\%\%\%\%\%\%\%\%\%\%\%\%\%\%\%\%\%\%\%\%\%\%\%\% $\%$ sets the fontweight and fontsize for current plot for a 5 inch wide picture in word \% \%\%\%\%\%\%\%\%\%\%\%\%\%\%\%\%\%\%\%\%\%\%\%\%\%\%\%\%\%\%\%\%\%\%\%\%\%\%\%\%\%\%\%\%\%\%\%\%\%\%\%\%\%\%\%\%\%\%\%\%\%\%\%\%\%\%\%\%\% $\% \operatorname{set}($ gca, 'fontweight', 'demi', 'fontsize',14);

\%\%\%\%\%\%\%\%\%\%\%\%\%\%\%\%\%\%\%\%\%\%\%\%\%\%\%\%\%\%\%\%\%\%\%\%\%\%\%\%\%\%\%\%\%\%\%\%\%\%\%\%\%\%\%\%\%\%\%\%\%\%\%\% $\%$ sets the fontweight and fontsize for current plot for a 6 inch wide picture in word \% \%\%\%\%\%\%\%\%\%\%\%\%\%\%\%\%\%\%\%\%\%\%\%\%\%\%\%\%\%\%\%\%\%\%\%\%\%\%\%\%\%\%\%\%\%\%\%\%\%\%\%\%\%\%\%\%\%\%\%\%\%\%\%\%\%\%\%\%\% $\% \operatorname{set}($ gca, 'fontweight', 'demi', 'fontsize',10);

title(\{['Output beam BL (resolution=', num2str(delta, '\%1.2f'), ', input Wo $=$ ', num2str(Wo, '\%4. $\left.0 f^{\prime}\right), \ldots$

' microns, shift= ', num2str(Primaryshift/(2), '\%3.of'),' microns)']; ['(focal distance $=1$, num2str $(\mathrm{df}, \ldots$

'\%4.Of'),' um, desired output Wo=', num2str(Wo2,'\%4.0f'),' microns)']\});

xlabel ('X (um)');

ylabel( 'Y (um)');

zlabel('Normalized Intensity');

\%\%\%\%\%\%\%\%\%\%\%\%\%\%\%

$\%$ Plot side view \%

$\% \% \% \% \% \% \% \% \% \% \% \% \%$

$\%$ sets plot window to close to full size

figure('Position', [scrsz(1)+4 scrsz(2) scrsz(3) scrsz(4)-45], 'color', [1 11 1])

mesh(posx, posy, Imax2, 'Facecolor', 'interp', ....

'FaceLighting', 'phong', 'Linestyle', 'none',' 'EdgeLighting', 'phong' );

\%\%\%\%\%\%\%\%\%\%\%\%\%\%\%\%\%\%\%\%\%\%\%\%\%\%\%\%\%\%\%\%\%\%\%\%\%\%\%\%\%\%\%\%\%\%

$\%$ To print just a portion of the array and save memory \% \%\%\%\%\%\%\%\%\%\%\%\%\%\%\%\%\%\%\%\%\%\%\%\%\%\%\%\%\%\%\%\%\%\%\%\%\%\%\%\%\%\%\%\%

$\%$ CENTER \% 


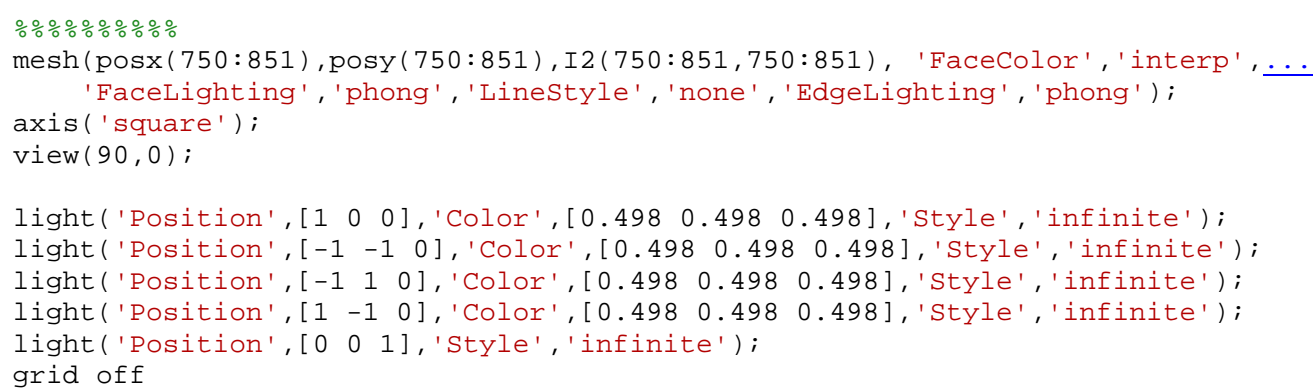




\section{Appendix C: AZ5214E}

The following data sheet was distributed by Clariant and was found at [37]

\begin{tabular}{|l}
\hline Product Data Sheet \\
AZ 5214 E \\
Image Reversal \\
Photoresist \\
Ims
\end{tabular}




\section{GENERAL INFORMATION}

This special photoresist is intended for lift-off-techniques which call for a negative wall profile. Although they are positive photoresists (and may even be used in that way) comprised of a novolak resin and naphthoquinone diazide as photoactive compound (PAC) they are capable of image reversal (IR) resulting in a negative pattern of the mask. In fact AZ 5214E is almost exclusively used in the IR-mode.

The image reversal capability is obtained by a special crosslinking agent in the resist formulation which becomes active at temperatures above $110^{\circ} \mathrm{C}$ and - what is even more important - only in exposed areas of the resist. The crosslinking agent together with exposed PAC leads to an almost insoluble (in developer) and no longer light sensitive substance, while the unexposed areas still behave like a normal unexposed positive photoresist. After a flood exposure (no mask required) this areas are dissolved in standard developer for positive photoresist, the crosslinked areas remain. The overall result is a negative image of the mask pattern.

As everybody knows a positive photoresist profile has a positive slope of $75-85^{\circ}$ depending on the process conditions and the performance of the exposure equipment (only submicron-resists get close to $90^{\circ}$ ). This is mainly due to the absorption of the PAC which attenuates the light when penetrating through the resist layer (so called bulk effect). The result is a higher dissolution rate at the top and a lower rate at the bottom of the resist. When AZ 5214E is processed in the IR-mode this is reversed as higher exposed areas will be crosslinked to a higher degree than those with lower dose, dissolution rates accordingly. The final result will be a negative wall profile ideally suited for lift-off.

The most critical parameter of the IR-process is reversal-bake temperature, once optimised it must be kept constant within $\pm 1^{\circ} \mathrm{C}$ to maintain a consistent process. This temperature also has to be optimised individually. In any case it will fall within the range from 115 to $125^{\circ} \mathrm{C}$. If IR-temperature is chosen too high $\left(>130^{\circ} \mathrm{C}\right)$ the resist will thermally crosslink also in the unexposed areas, giving no pattern. To find out the suitable temperature following procedure is suggested:

Coat and prebake a few substrates with resist. Without exposing them to UV-light subject them to different reversal-bake temperatures, i.e. $115^{\circ}, 120^{\circ}, 125^{\circ}$ and $130^{\circ} \mathrm{C}$. Now apply a flood exposure of $>$ $200 \mathrm{~mJ} / \mathrm{cm}^{2}$ and afterwards immerse them into a standard developer make up, i.e. AZ 351B, 1:4 diluted, or AZ $726 \mathrm{MIF}$ for 1 minute. From a part of the substrates the resist will be removed, another part (those exposed to a too high temperature) will remain with the resist thermally crosslinked on it. Optimum RBtemperature now is $5^{\circ}$ to $10^{\circ} \mathrm{C}$ below the temperature where crosslinking starts.

The flood exposure is absolutely uncritical as long as sufficient energy is applied to make the unexposed areas soluble. $200 \mathrm{~mJ} / \mathrm{cm}^{2}$ is a good choice, but $150-500 \mathrm{~mJ} / \mathrm{cm}^{2}$ will have no major influence on the performance.

Finally it should be noted that the imagewise exposure energy is lower than with normal positive processes, generally only half of that. So a good rule of thumb is: compared to a standard positive resist process, imagewise exposure dose should be half of that, flood exposure energy double of that for $A Z$ 5214E IR-processing.

Once understanding and being familiar with this IR-procedure it is quite simple to set up a different process for lift-off. A T-shaped profile can be achieved by the following process sequence:

The prebaked AZ 5214E photoresist is flood exposed (no mask) with a small amount of UV energy, just to generate some exposed PAC at the surface. Now the reversal-bake is performed to partially crosslink this top areas. By this treatment a top layer with a lowered dissolution rate compared to the bulk material is generated. After this the resist is treated like a normal positive photoresist (imagewise exposure and development) to generate a positive image! Due to the lower dissolution rate in the top layer a T-shaped profile with overhanging lips will be the result. 
PHYSICAL and CHEMICAL PROPERTIES

\begin{tabular}{|c|c|c|}
\hline & AZ 5214E & \\
\hline Solids content $[\%]$ & 28.3 & \\
\hline Viscosity [cSt at $\left.25^{\circ} \mathrm{C}\right]$ & 24.0 & \\
\hline Absorptivity $\left[\mathrm{lgg}^{*} \mathrm{~cm}\right]$ at $377 \mathrm{~nm}$ & 0.76 & \\
\hline Solvent & \multicolumn{2}{|c|}{ methoxy-propyl acetate (PGMEA) } \\
\hline Max. water content [\%] & \multicolumn{2}{|c|}{0.50} \\
\hline Spectral sensitivity & \multicolumn{2}{|l|}{$310-420 \mathrm{~nm}$} \\
\hline Coating characteristic & \multicolumn{2}{|l|}{ striation free } \\
\hline Filtration [um absolute] & \multicolumn{2}{|l|}{0.1} \\
\hline
\end{tabular}

\section{FILM THICKNESS $[\mu \mathrm{m}]$ as FUNCTION of SPIN SPEED (characteristically)}

\begin{tabular}{|l|c|c|c|c|c|}
\hline spin speed [rpm] & 2000 & 3000 & $\mathbf{4 0 0 0}$ & 5000 & 6000 \\
\hline AZ 5214E & & & & & \\
\hline & 1.98 & 1.62 & $\mathbf{1 . 4 0}$ & 1.25 & 1.14 \\
\hline
\end{tabular}

\section{PROCESSING GUIDELINES}

\begin{tabular}{|l|l|}
\hline Dilution and edge bead removal & AZ EBR Solvent \\
\hline Prebake & $110^{\circ} \mathrm{C}, 50^{\prime \prime}$, hotplate \\
\hline Exposure & broadband and monochromatic h- and i-line \\
\hline Reversal bake & $120^{\circ} \mathrm{C}, 2$ min., hotplate (most critical step) \\
\hline Flood exposure & $>200 \mathrm{~mJ} / \mathrm{cm}^{2}$ (uncritical) \\
\hline Development & $\mathrm{AZ} 351 \mathrm{~B}, 1: 4$ (tank, spray) or AZ 726 (puddle) \\
\hline Postbake & $120^{\circ} \mathrm{C}, 50$ s hotplate (optional) \\
\hline Removal & $\mathrm{AZ} 100$ Remover, conc. \\
\hline
\end{tabular}

\section{HANDLING ADVISES}

Consult the Material Safety Data Sheets provided by us or your local agent!

This AZ Photoresists are made up with our patented safer solvent PGMEA. They are flammable liquids and should be kept away from oxidants, sparks and open flames.

Protect from light and heat and store in sealed original containers between $0^{\circ} \mathrm{C}$ and $25^{\circ} \mathrm{C}$, exceeding this range to $-5^{\circ} \mathrm{C}$ or $+30^{\circ} \mathrm{C}$ for 24 hours does not adversely affect the properties

Shelf life is limited and depends on the resist series. The expiration date is printed on the label of every bottle below the batch number and coded as [year/month/day]

AZ Photoresists are compatible with most commercially available wafer processing equipment. Recommended materials include PTFE, stainless steel and high-density poly-ethylene and -propylene. 
The information contained herein is, to the best of our knowledge, true and accurate, but all recommendations are made without guarantee because the conditions of use are beyond our control. There is no implied warranty of merchantability or fitness for purpose of the product or products described here. In submitting this information, no liability is assumed or license or other rights expressed or implied given with respect to any existing or pending patent, patent application, or trademarks. The observance of all regulations and patents is the responsibility of the user.

A.Z, the AZ logo, BARLi, Aquatar and Kallista are registered trademarks of Clariant AG.

\begin{tabular}{|c|c|c|c|}
\hline Clariant $\mathrm{GmbH}$ & Clariant Corporation & Clariant (Japan) K.K. & Clariant Industries Ltd. \\
\hline Business Unit & Business Unit & Business Unit & Business Unit \\
\hline Electronic Materials & Electronic Materials & Electronic Materials & Electronic Materials \\
\hline Rheingaustrasse 190 & 70 Meister Avenue & 9F Bunkyo Green Court Center & 84-7, Chungdam-dong, \\
\hline Germany & USA & Tokyo 113, Japan & Seoul Republic of Korea \\
\hline Tel. +49 (611) 962-6867 & Tel. +1 (908) 429-3500 & Tel. +81 (3) $5977-7973$ & Tel. +82 (2) $510-8000 / 8442$ \\
\hline
\end{tabular}
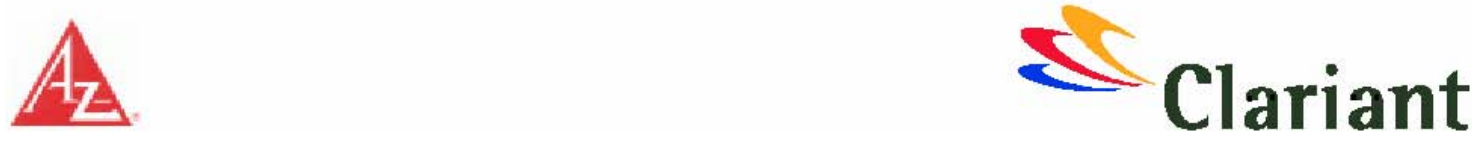


\section{Bibliography}

[1] M. Gad-el-Hak (editor), The MEMS Handbook, ISBN 0849300770, CRC Press, New York, 2002.

[2] Sandia National Laboratories SUMMiT V Process, http://www.sandia.gov/mstc/technologies/micromachines/tech-info/technologies/summit5.html

[3] http://www.memx.com/

[4] MEMSCAP MUMPs Foundry, http://www.memscap.com/memsrus/crmumps.html

[5] H. Li, J. Gaspar, V. Chu, J.P. Conde, P.P. Freitas, "MEMS microbridge deflection monitoring using integrated spin valve sensors and micromagnets", IEEE International Magnetics Conference, Digest of Technical Papers, April 28-May 2, 2002, pp GE5

[6] K. Hosokawa, R. Maeda, "In-line pressure monitoring for microfluidic devices using a deformable diffraction grating", The 14th IEEE International Conference on Micro Electro Mechanical Systems, Jan. 21-25, 2001, pp. 174-177.

[7] J. Park, L. Wang, J. Dawson, L. Hornak, P. Famouri, "Microstructure torque estimation using MEMS optical monitoring”, Proceedings of IEEE Sensors, v 2 , June 12-14, 2002, pp 1746-1750.

[8] S. Vardhan, M. Wilczynski, G.J. Portie, W.J. Kaiser, "Wireless integrated network sensors (WINS): distributed in situ sensing for mission and flight systems", IEEE Aerospace Conference Proceedings, v 7 March 18-25, 2000, pp 459 - 463.

[9] K.S. Brown, "On the Feasibility of Integrated Optical Waveguide-Based In-Situ Monitoring of Microelectromechanical Systems (MEMS)”, PhD. Dissertation, West Virginia University (2000).

[10] Carnegie Mellon ASIMPS, http://www.ece.cmu.edu/ mems/projects/asimps/index.shtml .

[11] J.M. Dawson, “Integrated Through-Wafer Monitoring of MEMS for Closed-Loop Control”, PhD. Dissertation, West Virginia University (2002).

[12] J. M. Dawson, J. Chen, K. S. Brown, P. Famouri, L. A. Hornak, “Through-wafer optical probe characterization for microelectromechanical systems positional state monitoring and feedback control”, Optical Engineering, v 39, n 12, Dec. 2000, pp 3239-3246

[13] J.M. Dawson, L. Wang, W.B. McCormick, S.A. Rittenhouse, P.F. Famouri, and L.A. Hornak, "Integrated Optical Monitoring of MEMS for Closed-Loop Control," Proc. SPIE, Vol. 4983, pp. 3443, Jan. 2003

[14] J. M. Dawson, L. Wang, W. B. McCormick, S. A. Rittenhouse, P. F. Famouri, L. A. Hornak, "Integrated optical monitoring of MEMS for closed-loop control," Proc. SPIE MOEMS and Miniaturized Systems III, San Jose, CA, 2003, pp 34-43.

[15] J. M. Dawson, L. Wang, P. Famouri, L. A. Hornak, "Grating-enhanced through-wafer optical microprobe for microelectromechanical system high-resolution optical position feedback," Optics Letters, v 28, n 14, July 15, 2003, pp 1263-1265.

[16] S.A. Rittenhouse, "Diagnosis of Operational Changes in Microelectromechanical Systems via Fault Detection”, Masters Thesis, West Virginia University (2004).

[17] B.E.A. Saleh and M.C. Teich, Fundamentals of Photonics, ISBN 0471839655, Wiley-Interscience, New York, 1991.

[18] E. Hecht, Optics, 3rd edition, ISBN 0201838877, Addison-Wesley Pub Co, 1997.

[19] C.C. Davis, Lasers and Electro-Optic, ISBN 0521484030, Cambridge University Press 1996.

[20] D. Marcuse, Light Transmission Optics, ISBN 0442263090, Van Nostrand Reinhold Company Inc., New York, 1982. 
[21] A.E. Siegman, Introduction to Lasers and Masers, New York, McGraw-Hill, 1971.

[22] U.S. Inan and A.S. Inan, Engineering Electromagnetics, ISBN 0805344233?, Prentice Hall, 1998.

[23] S. Ramo, J.R. Whinnery, T. Van Duzer, Fields and Waves in Communication Electromagnetics, ISBN 0471585513, John Wiley \& Sons Inc. New York 1994.

[24] E. Hecht, Schaum's Outline of Optics, ISBN 0070277303, McGraw-Hill, 1975.

[25] R.A. Serway, Physics for Scientists and Engineers, 4th edition, ISBN 0030059321, Saunders College Publishing, 1997.

[26] B. Kress and P. Meyrueis, Digital Diffractive Optics, 1st edition , ISBN 0471984477, John Wiley \& Sons Ltd, West Sussex England 2000. pp. 2-3.

[27] The American National Standard for Telecommunications Telecom Glossary 2000 http://www.atis.org/tg2k/_diffraction.html .

[28] Example of diffraction in water http://sol.sci.uop.edu/ jfalward/soundinterference/waterdiffraction.jpg .

[29] M. Born, E. Wolf, Principles of Optics, $6^{\text {th }}$ edition, ISBN 0080264824, Pergamon Press Inc., Elsmford, New York, 1980.

[30] C. Mead, L. Conway, Introduction to VLSI Systems, ISBN 0201043580, Addison-Wesley Publishing Company, Inc, Reading Massachusetts, 1980.

[31] Schott North America website, Borosilicate33 chemical composition, http://www.us.schott.com/whitegoods/english/products/borofloat/attribute/chemical/index.html .

[32] Schott North America website, Borosilicate33 UV transmission properties, http://www.us.schott.com/whitegoods/english/products/borofloat/attribute/optical/images/transmit_u v_range.pdf .

[33] Schott North America website, Borosilicate33 IR transmission properties, http://www.us.schott.com/whitegoods/english/products/borofloat/attribute/optical/images/transmit ir range.pdf .

[34] Schott North America website, Borosilicate33 dispersion properties, http://www.us.schott.com/whitegoods/english/products/borofloat/attribute/optical/images/dispersion borofloat.pdf.

[35] S.A. Campbell, The Science and Engineering of Microelectronic Fabrication, 2nd edition, ISBN 0195136055, Oxford University Press, 2001, pp 183, 258-280.

[36] Hoechst Trade Publication, "AZ 5200 Positive Photoresists”.

[37] Clariant AZ5214E Datasheet, https://www.first.ethz.ch/Datasheets/Data_AZ5214E.pdf

[38] K.R. Williams, K. Gupta, M. Wasilik, "Etch Rates for Micromachining Processing - Part II”, Journal of Microelectromechanical Systems, Vol. 12, No. 6, pp 761-778, December 2003.

[39] X. Li, T. Abe, M. Esashi, "Deep reactive ion etching of Pyrex glass using $\mathrm{SF}_{6}$ plasma”, Sensors and Actuators A, Vol. 87, No. 3, pp 139-145, 2001.

[40] COHU Inc. Installation and Operation Instructions for 4810 Series Monochrome Frame Transfer CCD Cameras.

[41] Spiricon Neutral Density Data http://www.spiricon.com/techinfo/product_data/beam_sampling/lbs100.shtml.

[42] United Detector Technology Radiometer/Photometer Model 350 Operating Instruction Manual.

[43] The MathWorks Technical Note 1106: Memory Management Guide April 2003, http://www.mathworks.com/support/tech-notes/1100/1106.shtml . 